Universidad PolitéCNICA DE MADRID

Escuela TÉcnica Superior de Ingenieros Industriales

Blackbox Nonlinear Modeling and Stability Analysis of DC Electronic Power Converters in Microgrids

Tesis Doctoral

Airán Francés Roger

Ingeniero Industrial, Universidad Politécnica de Madrid 

Departamento de Automática, Ingeniería Electrónica e INFORMÁTICA INDUSTRIAL

Escuela TÉcnica Superior de Ingenieros Industriales

\title{
Blackbox Nonlinear Modeling and Stability Analysis of DC Electronic Power Converters in Microgrids
}

\author{
AUTOR \\ Airán Francés Roger \\ Ingeniero Industrial por la Universidad Politécnica de Madrid \\ DIRECTOR \\ Javier Uceda Antolín \\ Doctor Ingeniero Industrial por la Universidad Politécnica de Madrid
}





\section{Acknowledgements}

$\mathrm{T}$

hese four years of $\mathrm{PhD}$ have been an adventure for me, which would not have been possible without the help of many people, whom I would like to thank.

First, I would like to express my gratitude to the members of the committee of this doctoral dissertation, Óscar García, Roberto Prieto, Javier Sebastián, Pablo Zumel, and Paolo Mattavelli. Second, I would like to thank especially to my advisor, Javier Uceda, for his invaluable guidance, vision, and support along the research process, without his help this thesis would not have been possible. Furthermore, I would like to thank my advisor during the internship in the Aalborg University, Josep María Guerrero, and his team, Juan Carlos, Enrique, Amjad, among others, for their advice and support along my stay there. I am also very grateful to all the professors of the Centro de Electrónica Industrial (CEI) for all they have taught me and for being always open to help whenever you need it.

I would like to thank also to my fellow students from CEI for creating a great work environment, especially to my former flatmates, Jorge and Filip, with whom I had a great time, and Gabriel and Mónica, for making the day life at CEI very enjoyable. I hope to keep their friendship forever.

I would also like to thank my lifelong friends because they have been always there when I have needed them, Winter, Jorge, Gon, Noe, ...

I am extremely grateful to my family because they have supported me from day one, especially my parents, who have given me every opportunity to follow my passion and they have enjoyed and suffer more than me with my successes and failures. Also to my brother who always encourages me to go after my dreams and he is always there to help no matter what.

Finally, I would like to thank Anoushka for sharing her life with me, even if that means to help me practicing "boring" speeches where she does not understand a single word. Your support has made this path much easier. 



\section{Abstract}

$\mathrm{T}$

he electric power distribution system is expected to experience a profound redesign in the next decades. The need for a transition from a centralized system, based on large power stations consuming fossil fuels, to a decentralized system able to integrate a massive amount of renewable energy sources is being acknowledged by all sectors of society. This transformation is mainly driven by the growing concern about the scarcity of fossil fuels, the pollution generated by this kind of power plants, their effect on the climate change, and the cost of implementing these systems in isolated areas or developing countries.

The development in technologies like power electronics, communication systems, energy storage systems, or the ones involved in the Internet of Things concept, can facilitate this transition, providing the designers with tools for the integration and optimization of the system. Besides, strategies to boost its reliability, robustness, and security are also more feasible in this framework.

The concept of microgrid is very popular in this context, which refers to independent structures able to work either isolated or connected to the rest of the electric power distribution system. The microgrids allow the implementation of hierarchical architectures, which greatly simplify the management of this complex system. This strategy divides the problem in different layers, where the lower ones control the voltage and current levels, the middle ones provide ancillary services, and the upper ones define the power flows.

The integration of electronic power converters in the power distribution system is key to provide controllability and dynamic independence among the different parts of the system. They allow the integration of the different elements into the microgrids and allow an independent behavior of the different microgrids that shape the power distribution system. Besides, the progress in power electronics has made the researchers to consider a dc distribution within the microgrids. The advantages of using a dc distribution, instead of the traditional ac approach, are: its higher suitability to interface most of the modern devices, like energy sources, storage elements, and loads, the lower complexity of the electronic power converters, and the simplicity of the control strategies, avoiding frequency synchronization, problems due to harmonics, etc. Therefore, with a dc distribution a microgrid can benefit from lower number of ac/dc conversions, an increased reliability due to the lower number of switching elements in the converters, and a higher simplicity in the design and control of the converters. These advantages result in a lower cost and size of the overall system.

Microgrids are characterized by a highly variable environment. The power delivered by the renewable sources depends on the weather conditions, the storage elements depend on their state of charge, the presence or not of the grid highly influences the behavior of the system, and the power demand is also dependent on the needs of the users. This variability can be handled by the electronic power converters, which can integrate controls with different operation modes depending on the power state of the microgrid. However, 
the wide range of operation conditions and the different control strategies entail a difficult challenge in the dynamic analysis of the system.

It is well-known that the interconnection of stable power converters that have been designed individually can result in degraded dynamic behaviors or even instabilities. Consequently, it is very convenient to model the different converters and to have the capability to perform simulations of the overall dynamic behavior of the microgrid. These models can help the designer to check the compatibility of the microgrid with the standards, to design external filters if necessary, to assess the effect of line impedances, to design system-level protections, etc. Besides, this kind of structure usually integrates system-level controls in order to perform the tasks of the second and third control layers. The design and evaluation of these system-level controllers should be also possible by means of the models of the electronic power converters.

Traditional power-electronic-based power distribution systems usually have a fixed operating point and the controllers mostly react to disturbances to keep the controller variable close to the reference value. Therefore, small-signal models around the nominal operating point are able to represent the dynamic of the converters with good accuracy. In complex applications, often the systems are designed as a whole, creating ad hoc solutions with the knowledge of all the elements of the system. Therefore, detailed models can be used to perform the simulations before the physical installation. However, in case a massive deployment of microgrids is intended, the use of commercial-off-the-shelf converters is highly desirable, in order to decrease costs and to improve the time-to-market. The price to pay by the system designers of using commercial converters is that, due to confidentiality issues, the information about the internal details of the converters will not be available. In this scenario, the only options for the system designer are to perform the physical installation without a previous simulation and design of the complementary elements, as filters, protections, or system-level controllers based on the response of the system; or to use blackbox modeling techniques to identify behavioral models able to represent the dynamic behavior of the converters.

The objective of this thesis is to propose a methodology to design blackbox models of the electronic power converter able to be integrated in a dc microgrid architecture. These models should be able to account for the large-signal response of the converters in the wide range of operation conditions of this kind of system. They should also be able to reproduce the behavior of converters with different operation modes and the effect of system-level controllers into the dynamic behavior of the interconnected system. All in all, the goal is to provide the system designer with tools for assessing and designing the dc microgrids, consisting of commercial-off-the-shelf converters and all the elements associated with them, at simulation level.

The methodology to achieve these goals has consisted in, first, reviewing the state of the art about the topics related with the thesis. In particular, the topics of interest are: the proposed architectures and control strategies for dc microgrids, analytical and blackbox modeling approaches for dc electronic power converters, and dynamic interaction and stability analysis of dc power distribution systems. Second, to compare the performance of the existing blackbox modeling approaches in order to assess their capability to account for the expected phenomena in dc microgrids. Third, to propose new methods to improve the estimation of the blackbox models in the cases where the existing methods are not able to do it. Fourth, to assess the possibility of applying nonlinear stability analysis methods using the information obtained from the blackbox models. Finally, to combine the blackbox models in dc microgrids like architectures in order to estimate the dynamic behavior of the interconnected system. Furthermore, the integration of secondary control strategies and their effect on the dynamic of the system will be also addressed. 


\section{Resumen}

Se Ne espera que el sistema de distribución de potencia eléctrica experimente un profundo rediseño en las próximas décadas. La necesidad de una transición desde un sistema centralizado, basado en grandes centrales eléctricas consumiendo combustibles fósiles, hacia un sistema descentralizado capaz de integrar una enorme cantidad de fuentes de energía renovables, está siendo reconocida desde todos los sectores de la sociedad. Esta transformación está impulsada principalmente por la creciente preocupación por la escasez de combustibles fósiles, la polución generada por este tipo de centrales eléctricas, su efecto en el cambio climático y el coste de implementar este tipo de sistemas en zonas aisladas o países en desarrollo.

El desarrollo en tecnologías como la electrónica de potencia, sistemas de comunicación, sistemas de almacenamiento de energía o las relacionadas con el concepto del "internet de las cosas", puede facilitar esta transición, dotando a los diseñadores de herramientas para la integración y optimización del sistema. Además, estrategias para potenciar su fiabilidad, robustez y seguridad son también más factibles en este tipo de estructura.

El concepto de micro-red es muy popular en este contexto, el cual se refiere a estructuras independientes capaces de trabajar tanto de forma aislada como conectada al resto del sistema de distribución de potencia eléctrica. Las micro-redes permiten la implementación de arquitecturas jerárquicas, las cuales simplifican enormemente la gestión de este tipo de sistemas complejos. Esta estrategia divide el problema en diferentes capas, donde las más bajas controlan los niveles de tensión y corriente, las medias proporcionan servicios auxiliares y las altas definen los flujos de potencia.

La integración de convertidores de potencia electrónicos en el sistema de distribución de potencia es esencial para introducir controlabilidad e independencia dinámica entre las distintas partes del sistema. Éstos permiten la integración de los distintos elementos en las micro-redes y posibilitan un comportamiento independiente de las diferentes microredes que conforman el sistema de distribución de potencia. Asimismo, el progreso en la electrónica de potencia ha permitido a los investigadores considerar una distribución en corriente continua dentro de las micro-redes. Las ventajas de usar una distribución en corriente continua, en lugar del acercamiento en corriente alterna, son una mayor conveniencia en la integración de la mayoría de los equipos modernos, como fuentes de energía, elementos de almacenamiento y cargas, la menor complejidad de los convertidores de potencia electrónicos y la simplicidad de las estrategias de control, evitando la sincronización de la frecuencia, problemas derivados de los armónicos, etc. Por lo tanto, con una distribución en continua una micro-red se puede beneficiar de un número menor de conversiones alterna/continua, un incremento en la fiabilidad debida al menor número de elementos que conmutan en los convertidores y una mayor sencillez en el diseño y control de los convertidores. Estas ventajas resultan en un menor coste y tamaño del sistema en su conjunto.

Las micro-redes se caracterizan por un entorno altamente variable. La potencia sum- 
inistrada por las fuentes renovables depende de las condiciones medioambientales, los elementos de almacenamiento dependen de su estado de carga, la presencia o no de la red influencia fuertemente el comportamiento del sistema y la demanda de potencia también depende de las necesidades de los usuarios. Esta variabilidad puede ser gestionada por los convertidores de potencia electrónicos, los cuales pueden integrar controles con distintos modos de operación en función del estado de carga en la micro-red. Sin embargo, el amplio abanico de condiciones de operación y las distintas estrategias de control conllevan un difícil desafío en el análisis dinámico del sistema.

Es bien sabido que la interconexión de convertidores de potencia estables que han sido diseñados de manera individual puede resultar en comportamientos dinámicos degradados o incluso en inestabilidades. Consecuentemente, es muy conveniente modelar los distintos convertidores y tener la capacidad de realizar simulaciones del comportamiento dinámico de la micro-red. Estos modelos pueden ayudar al diseñador a comprobar la compatibilidad de la micro-red con los estándares, a diseñar filtros externos si fueran necesarios, a analizar el efecto de las impedancia de línea, a diseñar protecciones a nivel sistema, etc. Además, este tipo de estructuras normalmente integran controles nivel sistema para realizar las tareas de las capas de control secundarias y terciarias. El diseño y la evaluación de estos controles de nivel sistema debería ser posible a través de los modelos de los convertidores de potencia electrónicos.

Los sistemas tradicionales de distribución de potencia basados en electrónica de potencia normalmente tiene un punto de operación fijo y los controles principalmente reaccionan a perturbaciones para mantener la variable controlada cercana al valor de referencia. Por lo tanto, modelos de pequeña señal alrededor del punto de operación nominal son capaces de representar la dinámica de los convertidores con una buena precisión. En aplicaciones complejas, a menudo los sistemas son diseñados en su conjunto, creando soluciones ad hoc con el conocimiento de todos los elementos del sistema. Por lo tanto, modelos detallados pueden ser utilizados para realizar simulaciones previas a la instalación física. Sin embargo, si se pretende realizar un despliegue masivo de micro-redes, el uso de convertidores comerciales es muy deseable, con objeto de reducir costes y mejorar el tiempo de salida a mercado. El precio a pagar para los diseñadores de nivel sistema de usar convertidores comerciales es que, debido a cuestiones de confidencialidad, la información sobre los detalles internos de los convertidores no estará disponible. En este escenario, las únicas opciones para el diseñador del sistema es realizar una instalación física sin una previa simulación y diseño de los elementos complementarios, como filtros, protecciones o controles de nivel sistema basado en la respuesta del sistema; o usar técnicas de modelado en caja negra para identificar modelos comportamentales capaces de representar el comportamiento dinámico de los convertidores.

El objetivo de esta tesis es proponer una metodología para diseñar modelos en caja negra de los convertidores de potencia electrónicos capaces de ser integrados en una arquitectura de micro-red de corriente continua. Estos modelos deberían ser capaces de tener en cuenta la respuesta en gran señal de los convertidores en el amplio abanico de condiciones de operación de este tipo de sistema. Éstos deberían también ser capaces de reproducir el comportamiento de convertidores con distintos modos de operación y el efecto de los controles de nivel sistema en el comportamiento dinámico del sistema interconectado. Con todo, el objetivo es proporcionar al diseñador del sistema las herramientas para analizar y diseñar las micro-redes de corriente continua, compuestas de convertidores comerciales y todos los elementos asociados a ellos, a nivel de simulación.

La metodología para conseguir todos estos objetivos ha consistido en, primero, revisar el estado del arte sobre los temas relacionados con la tesis. En particular, los temas de interés son: las arquitecturas y estrategias de control propuestas para micro-redes de corriente continua, propuestas de modelado analíticas y en caja negra para convertidores de potencia 
electrónicos en corriente continua y análisis de interacciones dinámicas y estabilidad de sistemas de distribución de potencia en corriente continua. Segundo, comparar el desempeño de las propuestas existentes de modelado en caja negra para analizar su capacidad de tener en cuenta los fenómenos esperados en micro-redes de corriente continua. Tercero, proponer nuevos métodos para mejorar la estimación de los modelos en caja negra en los casos donde los métodos existentes no son capaces de hacerlo. Cuarto, analizar la posibilidad de aplicar análisis de estabilidad no lineales usando la información obtenida de los modelos en caja negra. Finalmente, combinar los modelos en caja negra en arquitecturas del tipo de las micro-redes de corriente continua para estimar el comportamiento dinámico del sistema interconectado. Asimismo, se abordará la posibilidad de integrar estrategias de control secundario y estudiar su efecto en la dinámica del sistema. 


\section{Contents}

$\begin{array}{ll}\text { Acknowledgements } & \text { i }\end{array}$

$\begin{array}{ll}\text { Abstract } & \text { iii }\end{array}$

Resumen $\quad$ v

Contents viii

List of Tables

List of Figures $\quad$ xiii

List of Acronyms xix

$\begin{array}{llr}\text { Part I Background } & 1\end{array}$

1 Introduction $\quad 3$

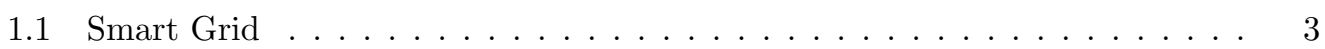

1.1 .1 AC vs DC Microgrids . . . . . . . . . . . . . . . 6

1.2 The need for accurate electronic power converter models . . . . . . . . . 7

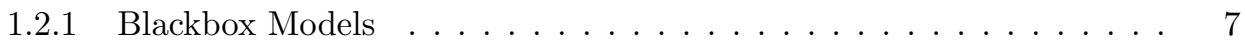

1.2 .2 Large-signal structures . . . . . . . . . . . . . . . . . . 7

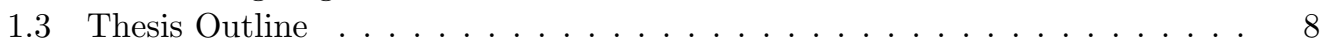

2 Study of the state of the art $\quad 11$

2.1 DC Microgrids . . . . . . . . . . . . . . . . . . . . . . . . . . . .

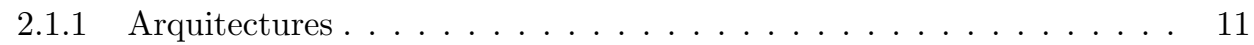

2.1 .2 Control techniques . . . . . . . . . . . . . . . 13

2.2 Modeling electronic power converters . . . . . . . . . . . . . . . . 17

2.2.1 Averaging techniques . . . . . . . . . . . . . . . . 17

2.2.2 Modular and nonlinear analytical modeling techniques . . . . . . . . 21

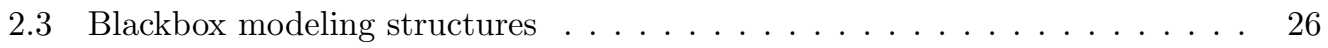

2.3 .1 Small-signal structure . . . . . . . . . . . . . . . . . . . . . . . . . . . . . .

$2.3 .2 \quad$ Large-signal structures . . . . . . . . . . . . . . . . . . . . 29

2.4 Dynamic interaction and stability analysis of dc-distributed systems . . . . 32

2.4.1 Impedance-based stability criteria . . . . . . . . . . . . . . . . 32

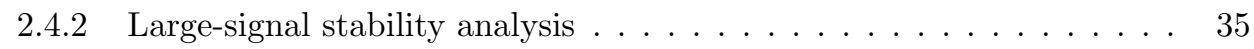

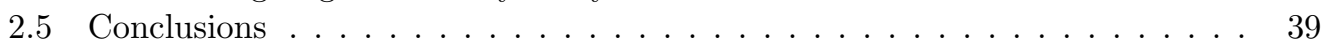


Part II Large-signal blackbox modeling of electronic power converters in Microgrids

3 Comparison of blackbox models of electronic power converters 43

3.1 Dependence of the converters on the operating point . . . . . . . . . . 43

3.1.1 Buck converter case . . . . . . . . . . . . . . . . . . . . . . . 44

3.1 .2 Boost converter case . . . . . . . . . . . . . . . . . . . . 54

3.1.3 Buck-boost converter case . . . . . . . . . . . . . . . . . . . . . 59

3.2 Converters with different operation modes . . . . . . . . . . . . . . . 64

3.3 Conclusions . . . . . . . . . . . . . . . . . . . . . . . . 68

4 Polytopic model with dynamic weighting functions $\quad 71$

4.1 Dynamic weighting functions . . . . . . . . . . . . . . 71

4.2 Voltage-controlled synchronous boost converter case . . . . . . . . . . . 73

4.3 Voltage-controlled buck converter in DCM and CCM case . . . . . . . . . 77

4.4 Voltage-controlled synchronous buck converter with double-loop control and current saturation case . . . . . . . . . . . . . . . . 80

4.5 Conclusions . . . . . . . . . . . . . . . . . . . . . . . . . 84

5 Parameter varying transfer functions with dynamic weighting functions 85

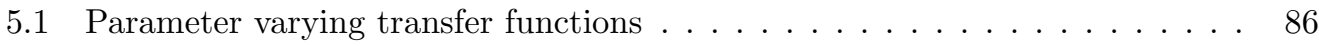

5.2 Voltage-controlled buck converter in DCM and CCM case . . . . . . . . 88

5.3 Voltage-controlled synchronous buck converter with double-loop control and

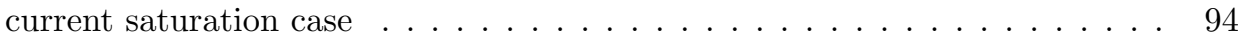

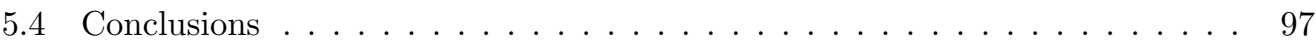

$6 \quad$ Large-signal blackbox stability analysis $\quad 99$

6.1 Application of the Lyapunov method to blackbox polytopic structures . . . . 100

6.1.1 Coincident polytopic models . . . . . . . . . . . . . . . . . . . . . . . . . 101

6.2 Constant power load example . . . . . . . . . . . . . . . . . . . 103

6.2 .1 Blackbox model . . . . . . . . . . . . . . . . . . . . . . . . . 104

6.2 .2 Linear stability analysis . . . . . . . . . . . . . . . . . . 107

6.2 .3 Large signal stability analysis . . . . . . . . . . . . . . . . . 109

6.3 Conclusions . . . . . . . . . . . . . . . . . . . . . . 111

7 DC Microgrid modeling $\quad 113$

7.1 Interconnection of blackbox models . . . . . . . . . . . . . . . 113

7.2 DC microgrid with dc bus signaling control . . . . . . . . . . . . . . 114

7.2.1 Inclusion of system-level control actions in the modeling structure . . 114

7.2.2 DC microgrid sources working with droop control and current limitation115

7.2.3 DC microgrid sources working with droop control, MPPT control, and system-level control strategies . . . . . . . . . . . . . . 119

7.3 Experimental validation: microgrid with droop control and system-level control strategies . . . . . . . . . . . . . . . . . . . . . . . . 128

7.4 Conclusions . . . . . . . . . . . . . . . . . . . . . . . . 137

8 Conclusions and Outlook 143

8.1 Summary of contributions . . . . . . . . . . . . . . . . . . 145

8.2 Future Work . . . . . . . . . . . . . . . . . . . . 146

8.3 Dissemination . . . . . . . . . . . . . . . . . . . . . . . . 147

8.3.1 Direct Contributions . . . . . . . . . . . . . . . . . . . 147

8.3.2 Other Works and Collaborations . . . . . . . . . . . . . . . . . . 147 
$\begin{array}{ll}\text { Part III Appendices } & 149\end{array}$

$\begin{array}{ll}\text { A Series connection of electronic power converters } & 151\end{array}$

B Analytical G-parameters model 153

B.1 Synchronous buck converter . . . . . . . . . . . . . . . . . 154

B.2 Synchronous boost converter . . . . . . . . . . . . . . . . 155

B.3 Synchronous buck-boost converter . . . . . . . . . . . . . . 158

C State-space representation of regulated converters $\quad 163$

C.1 Synchronous buck converter . . . . . . . . . . . . . . . 163

C.1.1 Evaluation of the equations for different operating points . . . . . 165

C.2 Synchronous boost converter . . . . . . . . . . . . . . 166

C.2.1 Evaluation of the equations for different operating points . . . . . . 168

C.3 Buck-boost converter . . . . . . . . . . . . . . . . 169

C.3.1 Evaluation of the equations for different operating points . . . . . 173

$\begin{array}{lr}\text { Bibliography } & 177\end{array}$ 


\section{List of Tables}

1.12030 Framework for Energy and Climate - Agreed headline targets. . . . . . . . 4

2.1 Characteristics of the most common dc voltages levels. . . . . . . . . . . . . . 14

2.2 Comparison of the capabilities of averaging techniques for dc EPCs. SSA (StateSpace Averaging), DF (Describing Function), GSSA (Generalized State-Space Averaging), CA (Cyclic-Averaging), DAM (Discrete Averaged Model). . . . . . .

2.3 Comparison of the capabilities of modular and nonlinear techniques for dc EPC systems. GP (G-parameters), CC (Component Connection), HM (Hybrid Modeling), LPV (Linear Parameter-Varying). . . . . . . . . . . . . . . 26

3.1 Parameters of the Wiener-Hammerstein model of the voltage-controlled synchronous buck converter . . . . . . . . . . . . . . . . . 46

3.2 Parameters of the identified transfer functions of the voltage-controlled buck converter for different values of the output current. . . . . . . . . . . . . . 50

3.3 Parameters of the Wiener-Hammerstein model of the voltage-controlled synchronous boost converter . . . . . . . . . . . . . . . . 57

3.4 Parameters of the Wiener-Hammerstein model of the voltage-controlled synchronous buck-boost converter. . . . . . . . . . . . . . . 61

3.5 Parameters of the identified transfer functions of the voltage-controlled buck converter with double-loop control in the two different modes of operation. . . .

5.1 Parameters of the identified transfer functions of the voltage-controlled buck converter for different values of the output current. . . . . . . . . .

5.2 Parameters of the identified transfer functions of the voltage-controlled buck converter for different values of the output current. . . . . . . . . . . . .

5.3 Parameters of the static weighting functions (SWF) implemented for the parameter varying transfer function model of the voltage-controlled buck converter in

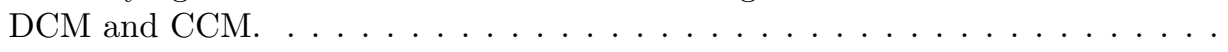

6.1 Parameters of the identified transfer functions of the source converter, corresponding to a unregulated synchronous buck converter simplified with its output

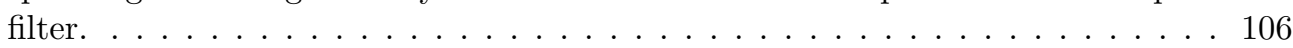

6.2 Evaluation of the input admittance of the simplified POL converter for different values of the output voltage of the source converter and a constant output power,

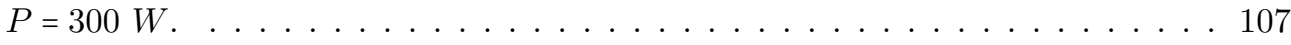

6.3 Parameters of the interconnected system using the identified G-parameters models of the converters. . . . . . . . . . . . . . . . . . 108

6.4 Parameters of the local state matrices obtained from the transfer functions identified after the similarity transformation. . . . . . . . . . . . . . 109

7.1 Identified data of the blackbox models. . . . . . . . . . . . . . . . 132 
C.1 Parameters of the transfer functions related to the output current of the voltagecontrolled boost converter for different operating points. . . . . . . . . . . 166

C.2 Parameters of the transfer functions related to the input voltage of the voltagecontrolled boost converter for different operating points. . . . . . . . . 167

C.3 Parameters of the transfer functions related to the output current of the voltagecontrolled boost converter for different operating points. . . . . . . . . . . . . 170

C.4 Parameters of the transfer functions related to the input voltage of the voltagecontrolled boost converter for different operating points. . . . . . . . . . . 171

C.5 Parameters of the transfer functions related to the output current of the voltagecontrolled buck-boost converter for different operating points. . . . . . . . . . 175

C.6 Parameters of the transfer functions related to the input voltage of the voltagecontrolled buck-boost converter for different operating points. . . . . . . . . . 176 


\section{List of Figures}

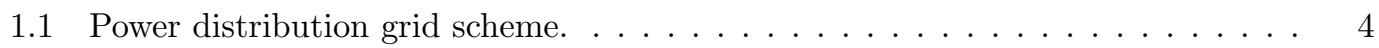

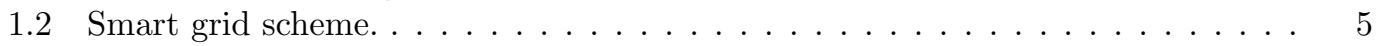

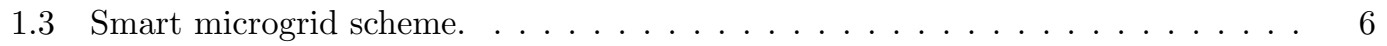

2.1 Single bus architectures . . . . . . . . . . . . . . . . . . . . . 12

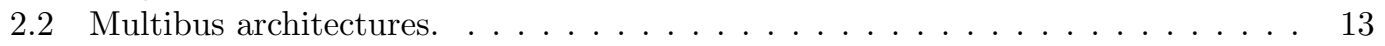

2.3 Droop control characteristic . . . . . . . . . . . . . . . . 16

2.4 DC Bus Signaling control. . . . . . . . . . . . . . . . . . . 16

2.5 Feedback system with a nonlinear element represented by a describing function. 24

2.6 G-parameters model. (a) Equivalent electrical circuit, (b) Block diagram notation. 28

2.7 Test procedure to identify the G-parameters model. (a) Load perturbation , (b) Source perturbation. . . . . . . . . . . . . . . . . . 28

2.8 (a) Wiener model, (b) Hammerstein model. . . . . . . . . . . . . . . . . . . 29

2.9 (a) Hammerstein-Wiener structure, (b) Wiener-Hammerstein structure. . . . . . 30

2.10 Oliver's Wiener-Hammerstein model. . . . . . . . . . . . . . . . . . . . 30

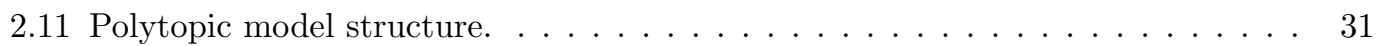

2.12 Forbidden regions. . . . . . . . . . . . . . . . . . . . . 33

3.1 Synchronous buck converter circuit. . . . . . . . . . . . . . . . . . . . . . 44

3.2 Comparison between switching model and G-parameters model of a synchronous buck converter in open loop. . . . . . . . . . . . . . . . . . . . . 45

3.3 Voltage-controlled synchronous buck converter circuit. . . . . . . . . . . . . . 46

3.4 Comparison between switching model and the three types of blackbox structures of a voltage-controlled synchronous buck converter. . . . . . . . . . . . 47

3.5 Error comparison between switching model and the three types of blackbox structures of a voltage-controlled synchronous buck converter. . . . . . . . . 48

3.6 Non-synchronous buck converter circuit. . . . . . . . . . . . . . . . . . . . 49

3.7 Frequency response of the buck converter for different values of the output current. Comparison between the measured signal and the identified transfer func-

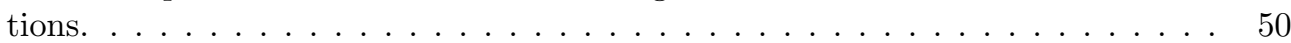

3.8 Comparison between switching model and the three types of blackbox structures of a voltage-controlled buck converter in DCM and CCM. . . . . . . . . . . 52

3.9 Error between the switching model and the three types of blackbox structures of a voltage-controlled buck converter in DCM and CCM. . . . . . . . . . . 53

3.10 Error comparison between switching model and the three types of blackbox structures of a voltage-controlled buck converter in DCM and CCM. . . . . . . 53

3.11 Synchronous boost converter circuit. . . . . . . . . . . . . . . . . 55

3.12 Comparison between switching model and G-parameters model of a synchronous boost converter in open loop. . . . . . . . . . . . . . . . . . . . . . . . . . 55

3.13 Voltage-controlled synchronous boost converter circuit. . . . . . . . . . . . 56

3.14 Comparison between the switching model and the three types of blackbox structures of a voltage-controlled synchronous boost converter. . . . . . . . . . 58 
3.15 Error comparison between the switching model and the three types of blackbox structures of a voltage-controlled synchronous boost converter. . . . . . . . . 59

3.16 Synchronous buck-boost converter circuit. . . . . . . . . . . . . . . . . 60

3.17 Comparison between the switching model and the G-parameters model of a synchronous buck-boost converter in open loop. . . . . . . . . . . . . . . 60

3.18 Voltage-controlled synchronous buck-boost converter circuit. . . . . . . . . . . . 61

3.19 Comparison between the switching model and the three types of blackbox structures of a voltage-controlled synchronous buck-boost converter. . . . . . . . . .

3.20 Error comparison between the switching model and the three types of blackbox structures of a voltage-controlled synchronous buck-boost converter. . . . . . . . 64

3.21 Voltage-controlled synchronous buck converter circuit with a double-loop control. 65

3.22 Frequency response of the voltage-controlled buck converter with double-loop control in voltage mode (VM) and in current mode (CM). Comparison between the measured signal and the identified transfer functions. . . . . . . . . . . 66

3.23 Proposed Wiener-Hammerstein model for voltage-controlled converters with doubleloop control and current limitation. . . . . . . . . . . . . . . 66

3.24 Comparison between switching model and the three types of blackbox structures of a voltage-controlled synchronous buck converter with a double-loop control. .

3.25 Error comparison between the switching model and the three types of blackbox structures of a voltage-controlled synchronous buck converter with a double-loop

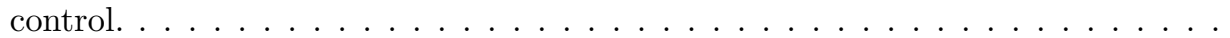

4.1 Scheme of the dynamic weighting function of one operating point defined by the

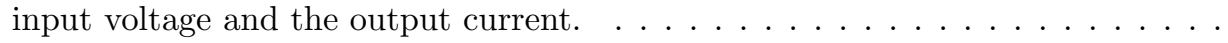

4.2 Comparison between the switching model and the polytopic model with the classical static weighting functions (SWF) and with the proposed dynamic weighting functions (DWF) for the case of a voltage-controlled boost converter. . . . . . . .

4.3 Error signals between the switching model and the polytopic model with the classical static weighting functions (SWF) and with the proposed dynamic weighting functions (DWF) for the case of a voltage-controlled boost converter. . . . . . .

4.4 Details about the performance of the polytopic model with the classical static weighting functions (SWF) and with the proposed dynamic weighting functions (DWF) for the case of a voltage-controlled boost converter. . . . . . . . . . .

4.5 Comparison between the switching model and the polytopic model with the classical static weighting functions (SWF) and with the proposed dynamic weighting functions (DWF) for the case of a voltage-controlled buck converter in DCM and CCM. . . . . . . . . . . . . . . . . . . .

4.6 Error signals between the switching model and the polytopic model with the classical static weighting functions (SWF) and with the proposed dynamic weighting functions (DWF) for the case of a voltage-controlled buck converter in DCM and

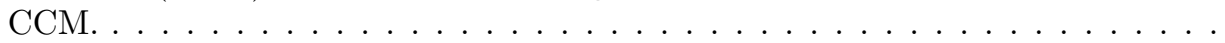

4.7 Details about the performance of the polytopic model with the classical static weighting functions (SWF) and with the proposed dynamic weighting functions (DWF) for the case of a voltage-controlled buck converter in DCM and CCM.

4.8 Scheme of the polytopic models with input-output variables and constant initial conditions (IC), Dynamic Weighting Function (DWF) and constant initial conditions, and DWF and variable initial conditions. . . . . . . . . . . .

4.9 Comparison between the switching model and the polytopic model with the classical static weighting functions (SWF), with the dynamic weighting functions (DWF), and with the dynamic weighting functions plus a variable initial condition of the output voltage for the model in current mode for the case of a voltage-controlled synchronous buck converter with a double-loop control. . . . . 
4.10 Details about the performance of the polytopic model with the classical static weighting functions (SWF), with the dynamic weighting functions (DWF), and with the dynamic weighting functions plus a variable initial condition of the output voltage for the model in current mode for the case of a voltage-controlled synchronous buck converter with a double-loop control. . . . . . . . . . . . .

5.1 Block diagram of a parameter varying transfer function with one zero and a pair of complex-conjugate poles. . . . . . . . . . . . . . .

5.2 Block diagram of the parameter varying transfer function of the output impedance for the voltage-controlled buck converter in DCM and CCM. . . . . . . . . . 88

5.3 Block diagram of the weighted combination of each parameter for the output impedance parameter varying transfer function. . . . . . . . . . . . . . 89

5.4 Block diagram of the dynamic weighting functions using a parameter varying transfer function. . . . . . . . . . . . . . . . . . . .

5.5 Block diagram of the parameter varying transfer functions model with DWF for the case of a voltage-controlled buck converter in DCM and CCM. . . . . . . .

5.6 Comparison between the switching model and the polytopic model with dynamic weighting functions (DWF) and the parameter varying transfer functions model with DWF for the case of a voltage-controlled buck converter in DCM and CCM. 92

5.7 Error between the switching model and the polytopic model with dynamic weighting functions (DWF) and the parameter varying transfer functions model with DWF for the case of a voltage-controlled buck converter in DCM and CCM. 93

5.8 Details about the performance of the polytopic model with dynamic weighting functions (DWF) and the parameter varying transfer functions model with DWF for the case of a voltage-controlled buck converter in DCM and CCM. . . . . . .

5.9 Block diagram of the parameter varying transfer function with for the synchronous buck converter with double-loop control with current saturation. . . .

5.10 Block diagram of the parameter varying transfer functions model with DWF for the case of a voltage-controlled buck converter with a double-loop control and current saturation. . . . . . . . . . . . . . . . .

5.11 Comparison between the switching model and the dynamic weighting functions plus a variable initial condition of the output voltage for the model in current mode and the parameter varying transfer functions (PVTF) model with dynamic weighting functions for the case of a voltage-controlled synchronous buck converter with a double-loop control. . . . . . . . . . . . . . .

5.12 Details about the performance of the dynamic weighting functions plus a variable initial condition of the output voltage for the model in current mode and the parameter varying transfer functions (PVTF) model with dynamic weighting functions for the case of a voltage-controlled synchronous buck converter with a double-loop control. . . . . . . . . . . . . . . . . . .

6.1 Negative impedance behavior of regulated POL converters. . . . . . . . . . . . 100

6.2 Scheme of a source converter supplying a POL converter connected to a resistive

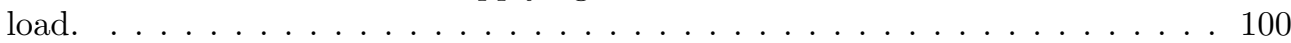

6.3 Scheme of the simplified circuit representing a cascade connection of a source converter supplying a POL converter that has a CPL behavior. . . . . . . . . . 103

6.4 Comparison between the frequency response of the source converter and the identified transfer functions. . . . . . . . . . . . . . . . . 105

6.5 Nyquist diagram of the minor-loop gain of the interconnected converter using the different local values of the input admittance of the POL converter. . . . . . 110

6.6 Regions of stability around the equilibrium point obtained using the whitebox (red) and blackbox (blue) approaches. . . . . . . . . . . . . . 110

6.7 State variables response with different initial conditions. . . . . . . . . . . . 111 
7.1 Taxonomy of the suitability of the different modeling approaches according to the dynamic behavior of the electronic power converters. . . . . . . . . . . 114

7.2 Scheme of the interconnection strategy among individual blackbox models. . . . 114

7.3 Scheme of the G-parameters model with the voltage reference as a new input and the inclusion of the droop control action. . . . . . . . . . . . . . 115

7.4 Scheme of the G-parameters model including the effect of the droop control in the static part of the model. . . . . . . . . . . . . . . . . 116

7.5 Studied de nanogrid scheme. . . . . . . . . . . . . . . 116

7.6 DC microgrid polytopic model scheme. . . . . . . . . . . . . . 116

7.7 Polytopic model performance for a regulated synchronous buck converter with a slow step from DCM to CCM. . . . . . . . . . . . . . . . 117

7.8 Polytopic model performance for a synchronous buck converter with sharp steps. 118

7.9 Polytopic model performance for a full-bridge converter using droop control and current limitation. . . . . . . . . . . . . . . . . . . . . . . 119

7.10 Polytopic model performance for a dc microgrid with droop control, current limitation and load disconnection. . . . . . . . . . . . . . . . 120

7.11 Microgrid scheme. . . . . . . . . . . . . . . . . . . 120

7.12 Small-signal model including the MPPT control effect to consider a variable power delivered by the RS. . . . . . . . . . . . . . . . 121

7.13 DC bus signaling strategy scheme. (a) Grid converter, (b) Renewable source converter, (c) Battery converter. . . . . . . . . . . . . . . . . 122

7.14 Implemented conditions to switch from the response of droop to MPPT controlled polytopic models. . . . . . . . . . . . . . . . . . 122

7.15 Performance of the proposed polytopic model for a buck converter using droopMPPT control strategies. . . . . . . . . . . . . . . . 123

7.16 Performance of the proposed simplified model to take into account changes in the maximum power from $P_{1}=150 \mathrm{~W}$ to $P_{2}=200 \mathrm{~W} \ldots \ldots \ldots \ldots$. . . . . . 124

7.17 Admittance (Y) parameters scheme. (a) Equivalent circuit, (b) block diagram

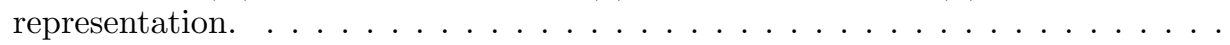

7.18 Performance of the proposed polytopic model for a system-level simulation with changes in the operation mode of the converters. . . . . . . . . . . 126

7.19 Performance of the proposed polytopic model to simulate the effect of the voltage restoration control technique. . . . . . . . . . . . . . . . 127

7.20 Performance of the polytopic model to simulate the current sharing control tech-

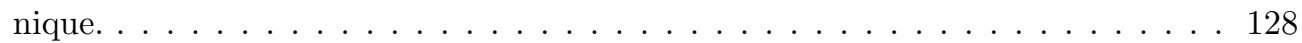

7.21 Experimental setup implemented. . . . . . . . . . . . . . . . . . 128

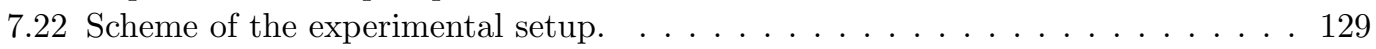

7.23 Scheme of the rectifier and its control structure. . . . . . . . . . . . 130

7.24 Sketch of the blackbox model of the rectifier. . . . . . . . . . . . . . 131

7.25 Sketch of the blackbox model of the dc-dc converter. . . . . . . . . . . . . . 132

7.26 Interconnection of the blackbox models. . . . . . . . . . . . . . . . 133

7.27 Simulation of how the microgrid dynamic behavior is affected by the parallel connection of the battery and grid interfacing converters. . . . . . . . . . . 134

7.28 Simulation of how the microgrid dynamic behavior is affected by the design of the current sharing secondary control. . . . . . . . . . . . . . 135

7.29 Comparison between the measured signals and the small-signal and the largesignal models response when the current sharing control is activated in low power conditions. LS: large-signal, HP: high power, LP: low power, OS: overshoot . . . 137

7.30 Comparison between the measured signals and the small-signal and the largesignal models response when the current sharing control is activated in medium power conditions. LS: large-signal, HP: high power, LP: low power, OS: overshoot.138

7.31 Experimental validation of the blackbox model of the microgrid using droop control. . . . . . . . . . . . . . . . . . . . . . . 139 
7.32 Experimental validation of the blackbox model of the microgrid in the activation of the current sharing. . . . . . . . . . . . . . . . . . . . . 140

7.33 Experimental validation of the blackbox model of the microgrid in the activation of the voltage restoration. . . . . . . . . . . . . . . . . . 141

A.1 Individual G-parameters models of two converters connected in series. . . . . . . 151

B.1 Synchronous buck converter circuit in open loop connected to a constant current

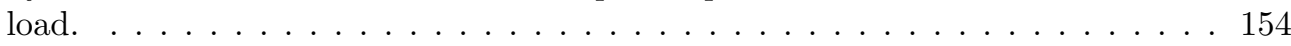

B.2 Variation of the frequency response of the G-parameters of the synchronous buck converter for different values of the duty cycle. . . . . . . . . . . . . 156

B.3 Synchronous boost converter circuit. . . . . . . . . . . . . . . . . . . . 156

B.4 Variation of the frequency response of the G-parameters of the synchronous boost converter for different values of the duty cycle. . . . . . . . . . . . . 159

B.5 Synchronous buck-boost converter circuit. . . . . . . . . . . . . . . 159

B.6 Variation of the frequency response of the G-parameters of the synchronous buck-boost converter for different values of the duty cycle. . . . . . . . . . . . . 161

C.1 Voltage-controlled synchronous Buck converter circuit. . . . . . . . . . . . . 163

C.2 Frequency response of the G-parameters of the voltage-controlled buck converter.165

C.3 Voltage-controlled synchronous Boost converter circuit. . . . . . . . . . . . . . . 166

C.4 Frequency response of the G-parameters of the voltage-controlled boost converter.169

C.5 Voltage-controlled synchronous Buck-boost converter circuit. . . . . . . . . . . . 172

C.6 Frequency response of the G-parameters of the voltage-controlled buck-boost

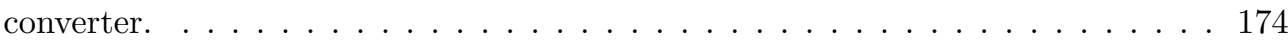





\section{List of Acronyms}

BDQLF Block-Diagonalized Quadratic Lyapunov Function

CA Cyclic-Averaging

CCM Continuous Conduction Mode

COTS Commercial-Off-The-Shelf

CPL Constant Power Load

DAB Dual Active Bridge

DAM Discrete Averaged Method

DBS DC Bus Signaling

DCM Discontinuous Conduction Mode

DF Describing Function

DWF Dynamic Weighting Function

EMI Electro-Magnetic Interference

EPC Electronic Power Converter

ESAC Energy Systems Analysis Consortium

ETSI European Telecommunications Standard Institute

EV Electric Vehicles

FQLF Full Quadratic Lyapunov Function

GMPM Gain Margin Phase Margin

GSSA Generalized State-Space Averaging

HCLF Hyper Cylindrical Lyapunov Function

HVDC High Voltage Direct Current

IEC International Electrotechnical Commission

KBM Krylov-Bogoliubov-Mitropolsky

LED Light-Emitting Diode 
LMI Linear Matrix Inequality

LPV Linear Parameter-Varying

LTI Linear Time-Invariant

LVDC Low Voltage Direct Current

MPC Maximum Peak Criterion

MPPT Maximum Power Point Tracking

POL Point-of-Load

PV PhotoVoltaic

PVTF Parameter Varying Transfer Functions

PWA Piece-Wise Affine

PWM Pulse Width Modulation

RESC Root Exponential Stability Criterion

ROA Region Of Attraction

SoC State of Charge

SSA State-Space Averaging

TIMF Time-Invariant Multi-Frequency 
Part I

Background 



\section{Introduction}

$\mathrm{T}$

his chapter describes the context and motivation of this thesis. First, the concept of "smart grid" is introduced, detailing its background, characteristics, and the challenges ahead. Second, the suitability of using blackbox approaches, as well as nonlinear structures, to model the Electronic Power Converter (EPC) in dc microgrids is discussed. Finally, the content of the thesis is outlined.

\subsection{Smart Grid}

The electric grid was conceived more than one hundred years ago. Its performance over the years has been excellent and it has been fundamental for the development of human activities around the world. Nowadays, our dependence to the grid is such that the costs due to blackouts have dramatic economic effects. Consequently, during the last years a large scale redesign of the power distribution system is being seriously considered in order to update it to the needs of the 21 st century.

Currently, the electric power system is unidirectional and the power balance between generation and consumption is achieved by turning on and off backup power plants, which are often less efficient, depending on the amount of power demanded. On the other hand, the dynamic of generation, distribution, and consumption is entirely coupled. The system stability is passively assured by the slow dynamic of the sources working at constant frequency. Therefore, the management of the constantly changing energy demands, the variability of renewable source energies, or power congestion is an issue. Furthermore, the integration of plug-in electric vehicles entails another important concern for the traditional system, as millions of new high-capacity batteries are expected to increase the power demand drastically and, probably, at the same time. These facts lead to waste of renewable energy and limitations in the integration of renewable energy sources and storage elements, over-designed systems and redundancy (Fig. 1.1).

Some of the main drivers towards a redesign of the electric power distribution system are the concern about the climate change, pollution, and dependence on fossil fuels. In December 2015, 195 countries agreed to the first world universal plan to tackle climate change by limiting global warming to 2 degrees Celsius above pre-industrial levels in the Paris agreement [1]. As part of the agreement each of the countries were requested to outline their post-2020 climate actions in what is known as Nationally Determined Contributions (NDCs). The agreement also includes the support to developing countries to take action 


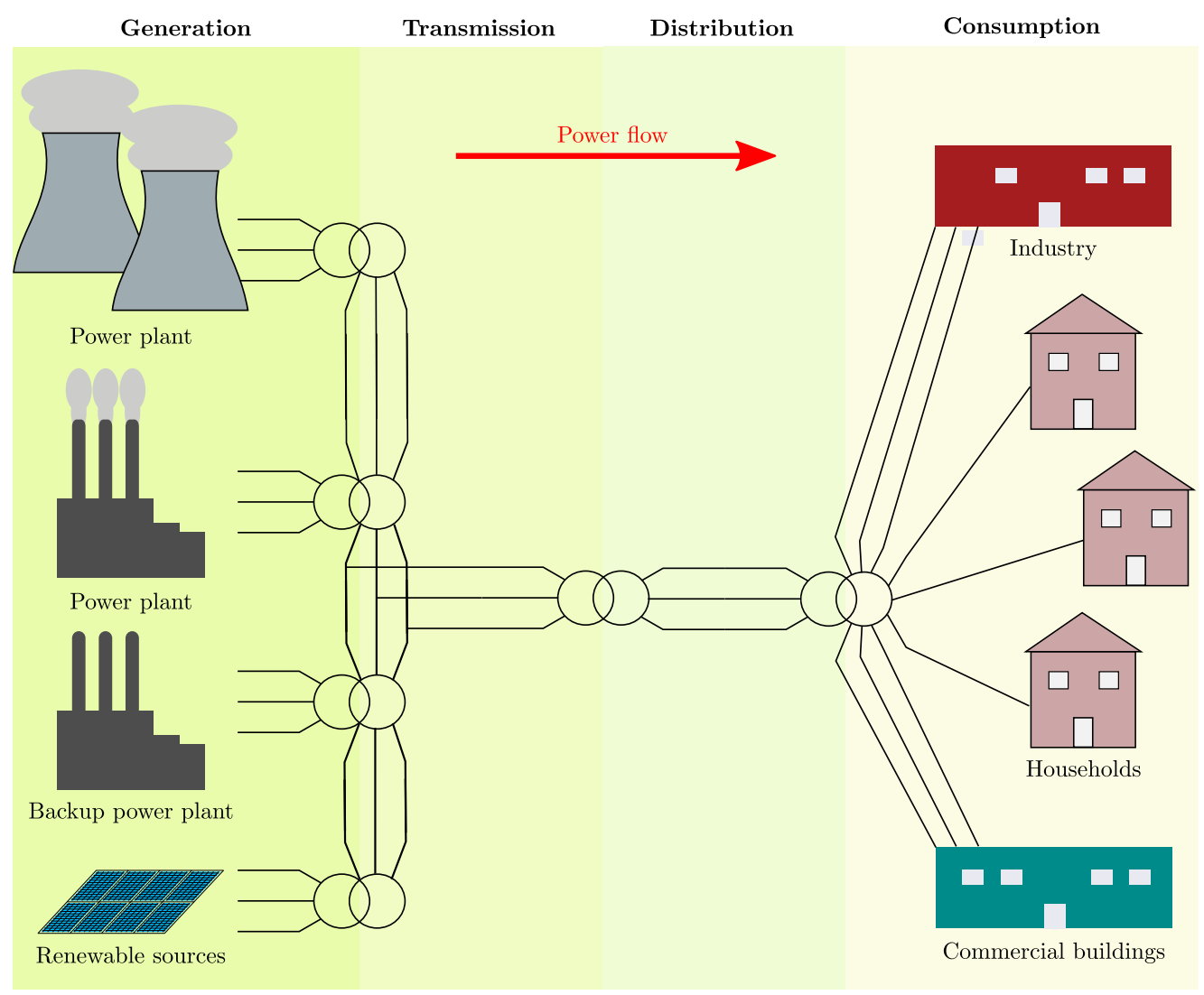

Figure 1.1: Power distribution grid scheme.

Table 1.1: 2030 Framework for Energy and Climate - Agreed headline targets.

\begin{tabular}{|c|c|c|c|c|}
\hline & $\begin{array}{l}\text { Greenhouse } \\
\text { Gas Emissions }^{1}\end{array}$ & $\begin{array}{l}\text { Renewable } \\
\text { Energy }\end{array}$ & $\begin{array}{l}\text { Energy } \\
\text { Efficiency }\end{array}$ & Interconnection \\
\hline 2020 & $-20 \%$ & $20 \%$ & $20 \%$ & $10 \%$ \\
\hline 2030 & $-40 \%$ & $27 \%$ & $30 \%$ & $15 \%$ \\
\hline
\end{tabular}

and adapt to climate change, what has been named as capacity-building. Consequently, in Europe, one of the ten priorities of the commission is the creation of the Energy Union [2] and, particularly, an energy strategy for 2020 and 2030 has set targets in four areas: greenhouse gas emissions, renewable energy, energy efficiency and interconnection (Table 1.1). In order to achieve these goals, Horizon 2020 will deploy over $€ 2$ billion in the period 2018-2020, with a special focus on: storage, renewables, buildings and (urban) e-mobility [3]. Furthermore, it is estimated that $€ 379$ billion will be required each year from 2021 onwards [4]. In the reports, the European Commission highlights the need to update the current grid infrastructure to make it smarter and able to deal with the high variability in these scenarios. A lot of effort has been put in creating a suitable regulation and standardization that facilitates the investment and integration of new technologies and to create a new open energy market where the users become an important part of the system.

The smart grid is a new paradigm in electric power distribution based on the application of the latest technological developments into the system [5]. The progress in power electronics, communications, computer science, automation, among others, brings new pos- 


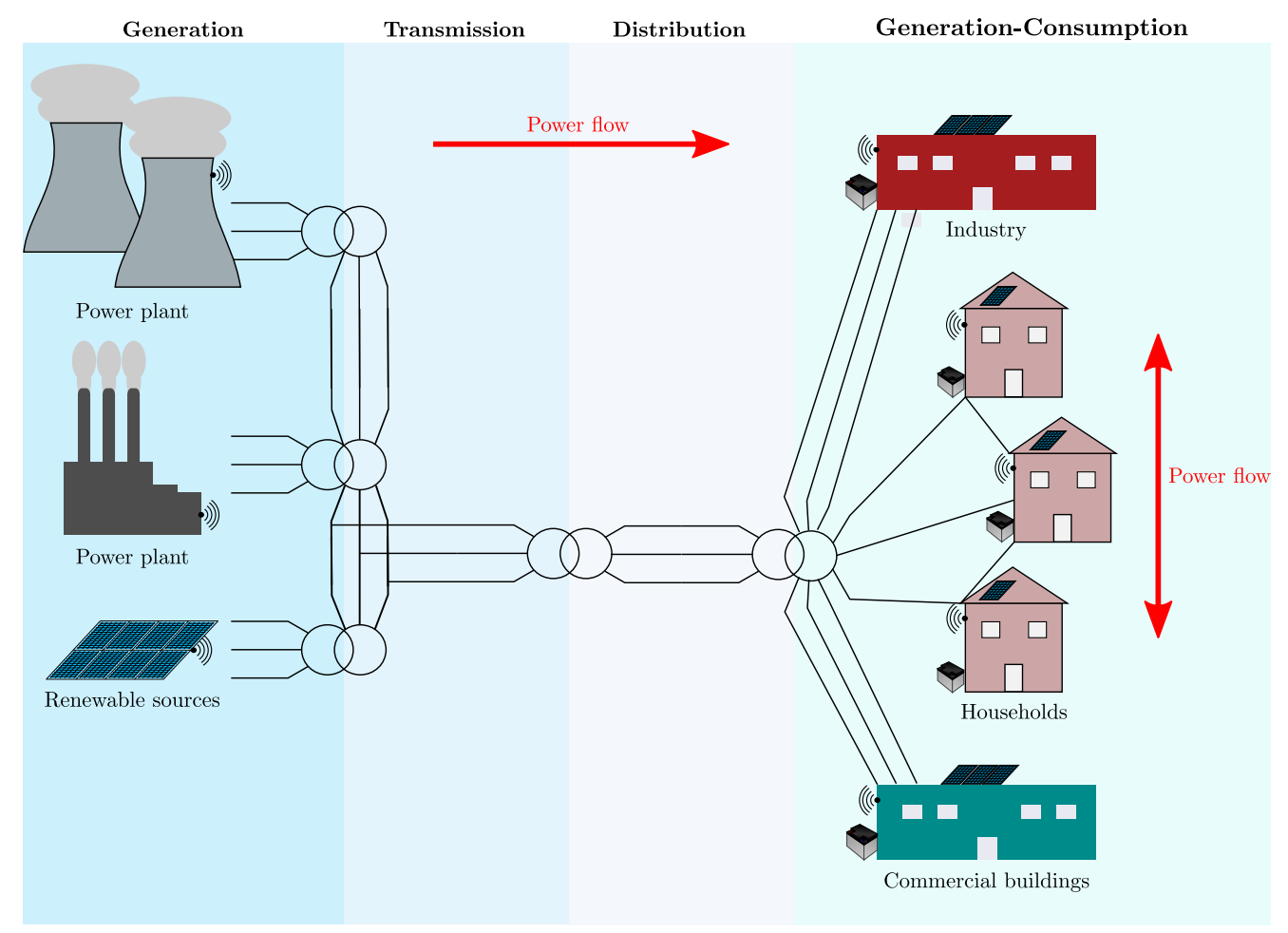

Figure 1.2: Smart grid scheme.

sibilities to make the grid more efficient, reliable, adaptive, and interactive. During the last decade, a vast amount of publications have been devoted to solve some of the aforementioned problems. Demand side management can help to deal with the gap between power generation and consumption by controlling non-critical loads [6]. The integration of distributed storage elements can also contribute to flatten the electric demand curve dealing with the variability of energy demand and renewable energy availability. The incorporation of distributed energy sources reduces losses in power transmission and allow the creation of a new energy market where the consumers become also providers (prosumers), which have many benefits in terms of adaptability, efficiency, exploitation of renewable sources, or robustness against blackouts $[7,8]$. The internet of things technology can provide meaningful information to optimize power flows, foresee problems, or improve planning [9-11]. Finally, big data, heuristic method and expert systems could be applied to manage large amounts of data and implement optimization algorithms [12] (Fig.1.2).

In this thesis, the focus will be on the advantages of implementing power electronics advancements to the electric power distribution system. The inclusion of EPCs provides controllability and dynamic decoupling. These characteristics are crucial to create the framework where the previous strategies can be implemented. On the one hand, the introduction of EPCs enables the division of the system in smaller independent parts, which can be interconnected and managed in a more efficient way. In the literature these individual portions of the smart grid have been called micro or nanogrids. In general, they are defined as parts of the system with local generation and/or storage and able to work either in grid-connected or in islanded/off-grid modes. On the other hand, power converters can interface every element of the microgrids (sources, storage elements, loads, different buses, other microgrids, etc.) offering the possibility to integrate communications, serve as power meters, optimize power flows, connection/disconnection, etc. (Fig. 1.3) 

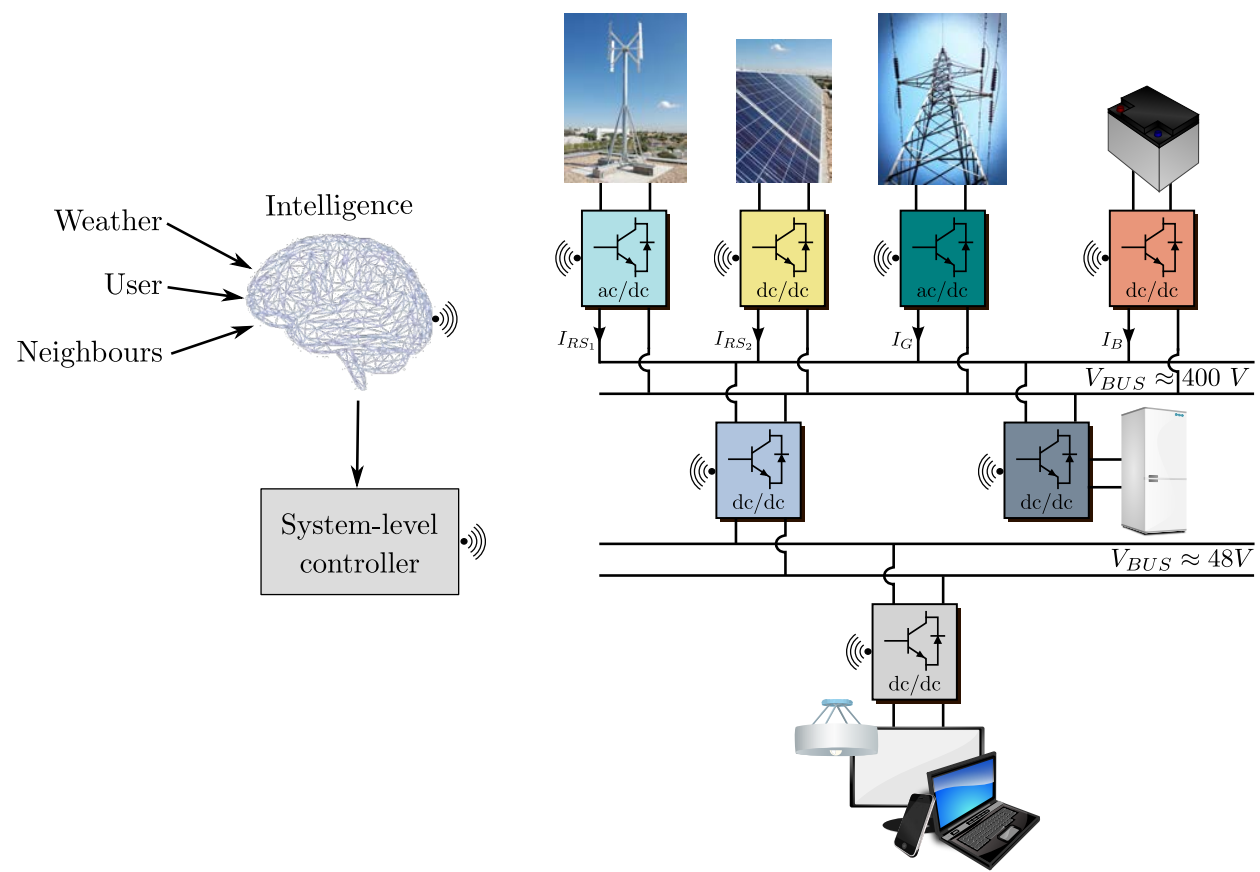

Figure 1.3: Smart microgrid scheme.

\subsubsection{AC vs DC Microgrids}

In the late 19th century, when the electric power grid was just starting to be created, an important decision had to be made: is it better to distribute the power in ac or dc? A general use of ac power was supported by Westinghouse and Tesla, whereas Edison favored a dc distribution. This was the beginning of "the war of the currents" [13]. The result was that the best option was to use ac power, which was motivated by the extensive use of the induction motors and the development of the transformer that made it simple to vary the voltage level. This decision has lasted until today.

Nowadays, the situation is different. The evolution in power electronic technology brings the possibility of upgrading the system toward the smart grid and the question about whether the electric power distribution should be in ac or dc has risen again [14]. Several works have been devoted to the performance comparison between these two options [15-17]. The decisive reason to use ac, i.e. easy modification of voltage level with transformers, is no longer a strong drawback for dc systems due to the development of $\mathrm{dc} / \mathrm{dc}$ converters. Some arguments in favor of the ac distribution are the maturity of the technology (protections, standards, etc.), the zero crossing of the voltage as an advantage to open the circuit (security), and the reliability of transformers to vary voltage levels. On the other hand, the use of a dc distribution has the benefits of simpler converters (smaller, cheaper, and more reliable), simpler controllers (avoid problems related with harmonics, reactive power, synchronization, etc.), and better interface with most of the elements of the system (renewable energy sources, storage devices, and most of the modern loads). A third possibility is to create hybrid microgrids with both ac and dc buses, taking advantage of the flexibility given by the EPCs [18]. The interest in dc distribution networks can also be found in the European Commission, reflected in several projects [19, 20].

Actually, dc systems are already all around us. PhotoVoltaic (PV) panels, batteries, Light-Emitting Diode (LED) lighting, Electric Vehicles (EV), electronic devices, etc. work in dc. Even ac systems as washing machines, refrigerators, fans, air conditioning systems, etc., are including dc motors to increase efficiency and controllability. DC distribution is 
being used in data centers, trams, telecom industry, Power Over Ethernet, USB, notebooks, etc. Also High Voltage Direct Current (HVDC) distribution systems are being deployed for transmitting large amounts of electricity over long distances and for special purpose applications.

A promising niche for dc power distribution is their deployment in developing countries. Around 1.2 billion people in the world do not have access to electricity and the suitability of dc distribution in local renewable energy based systems is generating a lot of interest. Finally, some standard organizations are also very interested in the implementation of Low Voltage Direct Current (LVDC) distribution systems for commercial and residential buildings, among other applications [21].

\subsection{The need for accurate electronic power converter models}

One of the main challenges of creating an EPC based distribution system is the fact that the interconnection of these devices can lead to dynamic degradation or even instabilities. The arrangement of the system in microgrids is a major advantage in this sense, as it allows an independent analysis from the rest of the system. Once the microgrid has been studied and the necessary precautions have been taken to ensure that their performance is acceptable under any predictable condition, they can be regarded as a single element for upstream analysis. The study of the interactions among the EPCs in microgrids is one of the main topics in this thesis.

An accurate model of the EPCs allows the study of dynamic interactions among the converters, but also helps to design system-level controllers, protections, external filters or to assess the compliance with the applicable standards. The main difficulties to obtain these models are the use of Commercial-Off-The-Shelf (COTS) converters and the presence of nonlinearities.

\subsubsection{Blackbox Models}

The vast majority of the models used in the literature are based on the analytical equations of the power converters. These models are fundamental in the design phase of the devices, as they aid in the component selection process and allow for sensitivity and stability analyses. Nevertheless, from the perspective of a dc microgrid designer, it would be cumbersome to make ad hoc designs for each application. The use of COTS converters is highly desirable to reduce the cost and the time-to-market of these projects. The price to pay in this case is that, due to confidentiality issues, manufacturers generally do not provide the users with enough information of their devices to produce an analytical model. Therefore, the only option to model the power converters is by using blackbox approaches.

Blackbox modeling refers to the design of dynamic models of a system whose internal details are unknown. The approach usually involves the application of system identification techniques to the response of the device under appropriate tests. This analysis can be performed in time domain, usually introducing step type perturbations, or in frequency domain, by means of bode plots.

In this thesis blackbox modeling techniques are used to account for the lack of details about EPCs. Some proposals are made in order to improve the performance of the existing methods and to take into account the specific phenomena that can arise in dc microgrids.

\subsubsection{Large-signal structures}

Traditionally, the study of interactions among power converters has been based on smallsignal analysis. Middlebrook was one of the first to notice that the stability of a power 
converter with an input filter can be assessed by just taking into account the impedances at their interface [22]. This method has been extensively used ever since, known as the Middlebrook stability criteria or the minor-loop gain. Later, this method was demonstrated to work not only for input filters, but also for the interconnection of power converters. Afterwards, it was noticed that the conditions that this author set to the minor-loop gain were very conservative and more sophisticated conditions were proposed. It is also worth noticing that this kind of admittance analysis not only gives information about the stability of the system, but also about the dynamic interactions among the converters.

This approach is very useful and simple, but it is important to have in mind that these methods rely on the small-signal approximation (as the equations must be linearized to obtain bode plots or represent the polar plot). Usually, dc power distribution systems work in a defined operating point and the regulators react to disturbances to keep it constant, so the small-signal approximation generally gives accurate results. However, dc microgrids are strongly variable systems due to the presence of renewable sources, fluctuating power demand, the state of charge of the batteries, and the possibility of working is islanded mode. This is one of the reasons why some authors propose the use of nonlinear controllers in this application. Furthermore, in order to deal with the parallel connection of sources and manage the power flow within the microgrid, often system-level controllers are suggested. Some of the system-level control strategies include changes in the operation mode of the converters depending on the power state of the microgrid.

In this context is reasonable to think that large-signal approaches might be necessary in order to take into account all the aforementioned scenarios. In this thesis large-signal techniques are implemented to model the power converters and to perform stability analysis.

\subsection{Thesis Outline}

The main ideas that motivate the topic of this thesis have been described above:

- The concern about climate change, pollution, and dependence on fossil fuels has created a global interest towards the use of renewable energies and a deep redesign of the current electric power system.

- The smart grid is a new paradigm in electric power distribution that facilitates a massive integration of renewable energy sources and new technologies to make the system more efficient, robust, secure and interactive.

- The integration of EPCs allow the creation of a framework where the smart grids are feasible.

- DC microgrids are an interesting alternative to ac microgrids, offering higher levels of simplicity and reliability, reduced cost and weight of the converters, and a better interface with most of the elements connected to the system.

- Blackbox approaches are an important tool to analyze interaction among the power converts in a COTS-based microgrid. Besides, these models can be used for designing system-level controllers, protections, external filters, and for checking the compliance with standards.

- Due to the strong variability present in the microgrids, large-signal approaches are expected to be a useful tool to account for the nonlinearities in the system. These nonlinearities can be present due to the operating point dependent behavior of the converters, the use of nonlinear controllers, controllers with different operation modes, etc. 
In particular, the specific research questions that will be addressed in this thesis are:

- is it possible to obtain blackbox models of the electronic power converters that are part of dc microgrids such that they are able to reproduce their dynamic behavior in all conditions?

- is it possible to perform large-signal stability analyzes from the information obtained from the blackbox models of the electronic power converters?

In this context, this thesis proposes a methodology to obtain accurate blackbox largesignal models of EPCs in dc microgrids, to analyze interactions among them, to design system-level controllers, and to perform stability analysis. It is divided in the following chapters:

- Chapter 2 studies the state of the art of dc microgrids, modeling techniques for EPCs, blackbox structures, and interaction and stability analyzes.

- Chapter 3 compares the performance of the existing blackbox modeling approaches in different illustrative scenarios. The dependence of the dynamic behavior of EPCs with the operating point is analyzed, as well as the capability of these modeling approaches to account for changes in the control mode of the converters.

- Chapter 4 introduces the concept of dynamic weighting functions applied to polytopic models. This is a method to improve the performance of the model to reproduce the dynamic behavior of the EPCs when the input variables have sharp variations.

- Chapter 5 proposes a new blackbox modeling approach in order to account for converters with strong nonlinearities and sharp changes in the input variables. As shown in Chapter 3, the dynamic of this kind of converters is not well reproduced by the modeling approaches available in the literature.

- Chapter 6 details the methodology to apply large-signal stability analysis from blackbox polytopic models. A Lyapunov method for analytical polytopic models is applied to the blackbox models obtained by means of identification methods.

- Chapter 7 presents the performance of the modeling approach proposed in different dc microgrid scenarios. Besides, system-level controllers are included in the blackbox modeling structures. Finally, an experimental validation is presented.

- Chapter 8 summarizes the contributions and the importance of the work presented in this context. 



\section{Study of the state of the art}

T

his chapter reviews the state of the art in the main topics related to this thesis, namely dc microgrids architectures and control strategies, analytical and blackbox modeling approaches, stability analysis of dc-distributed systems, and dynamic interaction among EPCs.

\subsection{Microgrids}

DC microgrids are currently being used in several different applications as: universal serial bus, power over ethernet, universal power adapter for mobile devices, vehicles in terrestrial, maritime, and aerospace applications, buildings with high-reliability needs such as hospitals, data centers, military facilities, or off-grid households in developing countries [23]. The main advantage of a local dc distribution is the better interface with renewable energy sources, storage systems, and most of modern loads, avoiding unnecessary ac/dc and dc/ac conversions. Also problems associated with ac distribution are avoided such as harmonics or frequency synchronization. This leads to simpler and more reliable systems with reductions in cost and size. In this section the state of the art in architectures and control techniques for dc microgrids is reviewed.

\subsubsection{Arquitectures}

Power electronic based distribution systems are mainly characterized by the presence of buses, where sources, storage devices, and loads are interconnected. Consequently, the number of buses, their electric characteristics, and the way they are interconnected are the main features that define this kind of power distribution architecture. Accordingly, the different alternatives that can be found in the literature can be classified in single and multibus topologies [24].

\section{Single-bus Architecture}

The use of only one bus is traditionally the most extended choice. This structure has the advantage of simplicity and it has been widely used in data centers due to their constant high power demand. A classic approach to ensure the stability of the interconnected system is to directly connect energy storage devices, typically electro-chemical batteries, to the bus, due to their high capacitance. However, the direct connection of batteries to the bus entails 


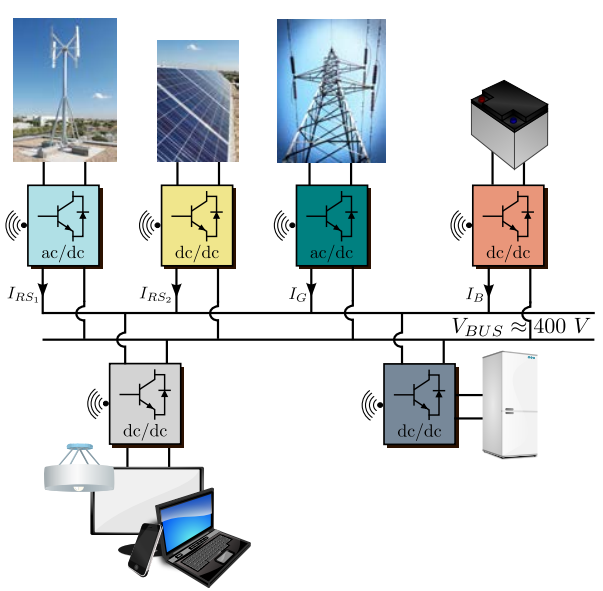

(a) Single bus architecture.

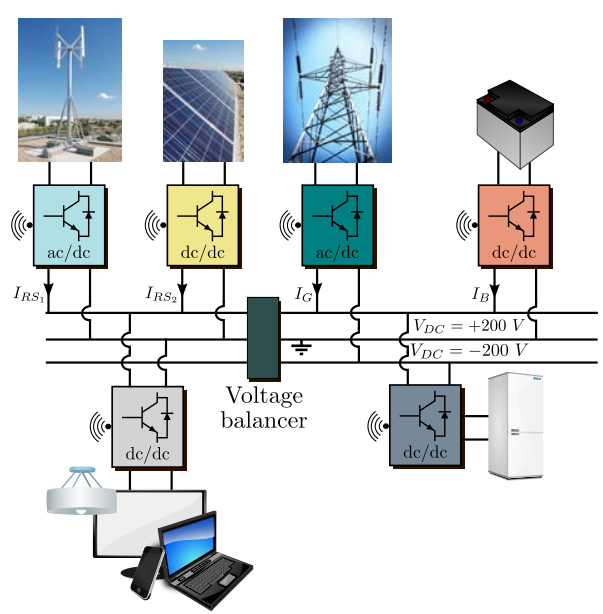

(b) Bipolar architecure.

Figure 2.1: Single bus architectures

several drawbacks. First, the bus voltage is not controllable and depends mainly on the State of Charge (SoC) and output current of the battery. Second, the charge of the battery has to be performed with parallel sources and measurement errors in the voltage value can generate circulating currents. Finally, safety is also an issue as connecting batteries directly to buses with voltages higher than a few hundred volts can lead to improper interruption of faults [25]. Therefore, the connection of all the elements through EPCs is often preferred in dc microgrid applications. The EPCs provide controllability to the systems to control power flows and to establish an optimum management of the batteries in order to maximize their lifetime. Nevertheless, the enhanced stability of the previous method is lost, so it should be perform by means of an adequate control strategy.

The main drawback of this architecture is that the same voltage level is set for high and low power devices, so the efficiency of the system is not optimum. A way to partially deal with this issue is to use bipolar structures [26]. This architecture is a three-wire composition, where the bus voltage includes a neutral point, so it is divided in $+V_{d c} / 2$ and $-V_{d c} / 2$. Therefore, the elements of the system can be connected to $V_{d c}$ using the positive and negative wires or to $V_{d c} / 2$ by connecting to either the positive or negative wire and the neutral point. In Fig. 2.1 these architectures are depicted.

\section{Multibus Architecture}

Multibus architectures consist of buses that may or may not have different voltage levels. The goal of using more than one voltage level is to create better interfaces for the different elements of the microgrid in order to enhance the overall efficiency of the system. High power devices are connected to high voltage buses in order to reduce conduction loses. However, most of the electronic loads work with much lower voltage levels. On the other hand, the presence of multiple buses enhance the reliability of the system, as faults can be isolated and the power rerouted through other paths [27]. Different kinds of connection strategies have been proposed to increase flexibility and reliability as dc ring configuration [28] or zonal configuration [29].

This structure is more complex but, similarly to what happens in notebook power distribution systems, it can lead to more efficient, lighter, reliable, and cheaper solutions, compared with single-bus architectures [30]. In Fig. 2.2 these architectures are depicted. 


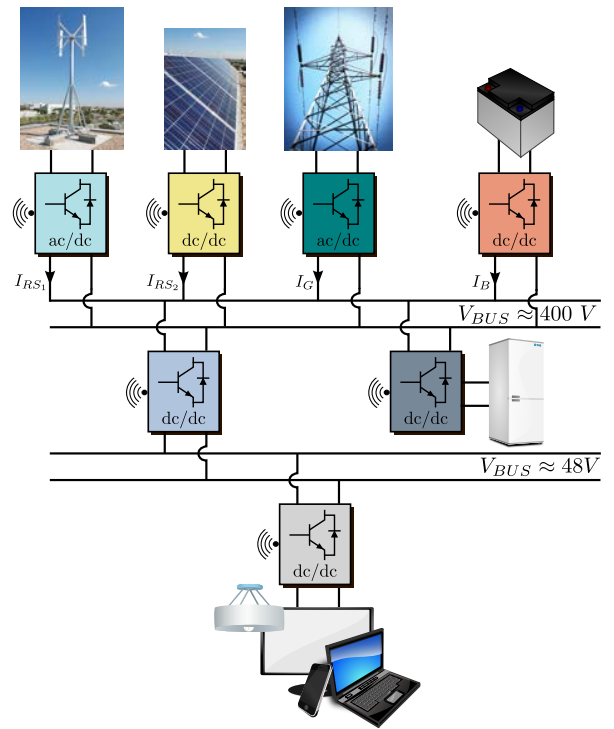

(a) Architecture with two voltage levels.

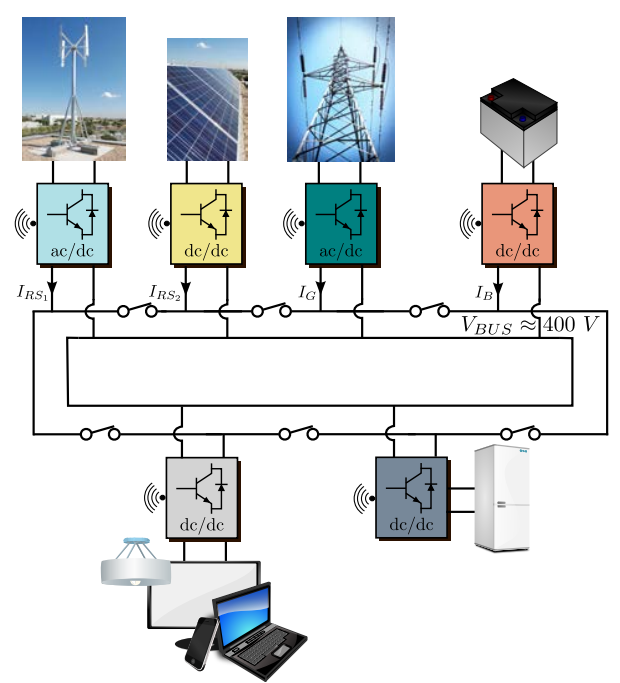

(b) DC ring architecture.

Figure 2.2: Multibus architectures.

\section{Bus Voltage Selection}

Currently there is a number of organizations working to create standards for dc microgrids in residential applications, such as the European Telecommunications Standard Institute (ETSI), EMerge Alliance, International Electrotechnical Commission (IEC), the IEEE, etc. [31]. There are several aspects to take into account to select an appropriate voltage level such as power delivered, safety, compatibility with existing standards for different applications, etc. Table 2.1 summarizes the characteristics of the most common dc voltages levels considered, the existing standards, and applications.

In the literature, the most extended selection is a multibus architecture with two or three voltage levels. A high voltage level $(380-400 \mathrm{~V})$ is often proposed to supply high power loads, whereas one or two low voltage levels (24-48 V) are often considered to supply low power loads and to enhance a safe plug\&play operation.

\subsubsection{Control techniques}

A dc microgrid consists of three main elements: sources, storage devices, and loads. The sources can be separated in the main grid, renewable sources, and possibly nonrenewable sources. The storage devices can be electromechanical batteries, super-capacitors, flywheels, etc. Regarding the loads, in general there are no distinctions but in some applications it is possible to find different levels of priority in case of scarce energy or fault.

Concerning the sources, it is desirable to extract the maximum energy from the renewable sources, which usually work with Maximum Power Point Tracking (MPPT) controllers [32]. However, in case the microgrid is not able to absorb the power available, some protective actions should be taken. The main grid often serves as a buffer that provides or absorbs energy from the system as needed. In a liberalized energy market scenario, the grid interface converter could set prices for the power delivered or consumed from the microgrid. The nonrenewable sources in general are only present for backup and their use should be limited to faulty modes.

In the case of energy storage systems, the main variable is their SoC. Optimization algorithms can be used to define the best moments to charge or discharge these devices. 
Table 2.1: Characteristics of the most common dc voltages levels.

Standard

$1500 \mathrm{~V}$

Limit of LVdc

(IEC60038)

$750 \mathrm{~V}$

$\geqslant 565 \mathrm{~V}$

$400 \mathrm{~V}$

Maximum telecom dc source level ETSI EN 300 132-3-1

$380 \mathrm{~V}$

EMerge Alliance (Data/Telecom Std)

$325 \mathrm{~V}$

$120 \mathrm{~V}$

Limit of SELV and PELV (IEC61140)

$75 \mathrm{~V}$

Low limit EU LDV 2014/35/EU

$50 \mathrm{~V}$

IEEE 802.3bu

802.3bt,

$48 \mathrm{~V}$

$24 \mathrm{~V}$

$$
\begin{aligned}
& \text { EMerge } \\
& \text { (occupied }
\end{aligned}
$$
Std)

Alliance
space

$12 \mathrm{~V}$
Application

Comment

Traction systems, PV systems

Trams power systems

Direct connection with $3 \phi 400 V_{a c}$ grid

EV fast charging

Data center
Compatible with load with input rectifier

No need for protections against indirect contacts

Power over Ethernet

Telecom, Rural PV systems, Trucks

Lighting systems, electric bikes, highpower cordless tools

Automotive, lighting 
Also, the way these elements are charged or discharged is important to maximize their lifetime. The control of the bus voltage can be performed by either the sources or the storage elements or in a coordinated way.

Finally, the ultimate goal is to keep all the loads supplied. However, in case the power available in the microgrid is not enough to supply the loads, some prioritizing techniques could be applied.

In order to achieve all these goals the interface converters must work in a coordinated way. There are three main approaches to perform this coordination: centralized, decentralized, and distributed controls.

\section{Centralized control}

In centralized approaches all the relevant parameters are sent to a central controller that performs the optimization algorithms and set the corresponding references to the converters. This is the best method to achieve an optimum behavior of the system, as the central controller is aware of all the pertinent information about the microgrid, and possibly some external information, to perform the optimization. A master/slave high-bandwidth communication is usually employed in this approach.

This control has two main drawbacks when applied to dc microgrids. First, it has a single point of failure as it relays on the correct operation of the central controller and the communication system. This problem could be partially solved by means of redundancy, however the cost and size of the system would be increased. Second, a single device is in charge of the entire computation burden. This aspect limits the number of elements included in the microgrid, complicates the inclusion of new elements, and it requires more complex devices to perform the necessary amount of calculations within the required period of time.

\section{Decentralized control}

Decentralized approaches, unlike the previous method, do not employ communication strategies among the converters. The bus voltage level is used as an indicator of the power state of the microgrid. This approach makes use of droop controllers to set a virtual impedance that regulates the power flows and also contributes to the stability of the system. In general, the higher the droop gains the better the current regulation and damping of the system, however the voltage deviation of the bus voltage increases.

Taking advantage of the relationship between the power state of the microgrid and the bus voltage, the DC Bus Signaling (DBS) control strategy has been proposed [33]. When droop controllers are used, a bus voltage higher than the nominal bus voltage means that the generation is higher than the consumption level, whereas a lower level of the bus voltage corresponds to the opposite case. The voltage reference of the controllers using droop control can be expressed as follows:

$$
V_{\text {ref }}=V_{n}-k_{d} I_{o}
$$

where $V_{\text {ref }}$ is the voltage reference, $V_{n}$ is the nominal voltage of the bus, $k_{d}$ is the droop parameter, and $I_{o}$ is the output current of the EPC. The V-I characteristic of a controller represented in Fig 2.3.

Therefore, the DBS technique defines intervals of the bus voltage where the controllers change their operation mode to deal with these circumstances. For instance, when the bus voltage is above a predefined level, the renewable sources will change from MPPT control to voltage control as the microgrid is not able to absorb all the power available in these sources. 


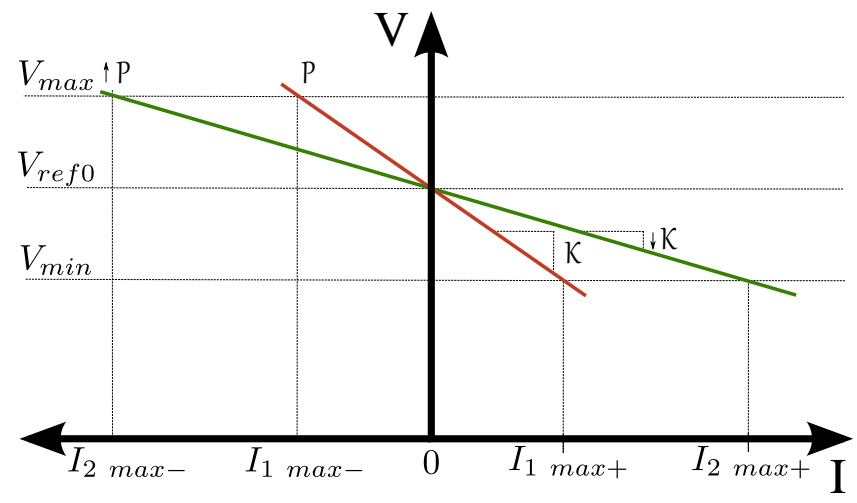

Figure 2.3: Droop control characteristic.
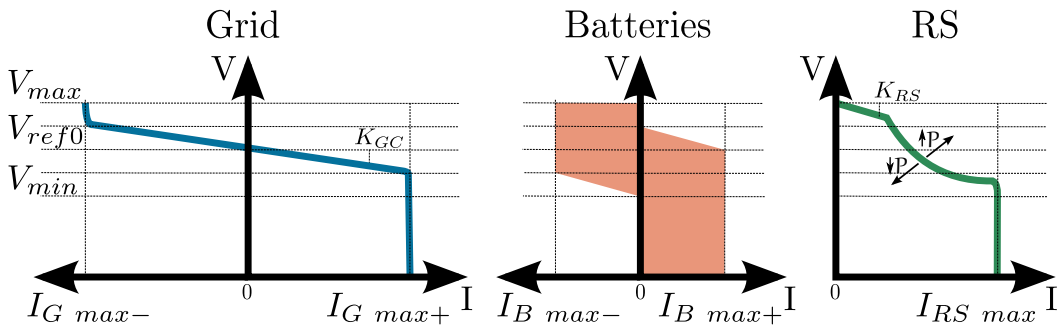

Figure 2.4: DC Bus Signaling control.

On the other hand, when the bus voltage is below its minimum level, the nonrenewable energy sources will be activated if they are available, or a load shedding strategy can be implemented in order to ensure the supply to critical loads. The main advantage of this strategy is its simplicity, flexibility, and expandability (Fig. 2.4).

Compared with centralized controllers, with this approach it is difficult to achieve an optimum behavior of the system due to the lack of information. However, in order to dynamically control the power flows within the microgrid, a low-bandwidth non-critical communication system could be included in order to modify the references of the converters. Another disadvantage of this strategy is that non-considered impedances in the cables and errors in voltage measurements can lead to inaccurate current control.

\section{Distributed control}

An intermediate approach between the previous two methods is the distributed control. In this strategy the EPCs communicate with their neighbors in order to improve the coordination among the different sources. Consensus algorithms have been proposed in order to achieve these goals $[34,35]$.

This strategy solves the main drawback of the previous methods, i.e. avoid a single point of failure and inaccuracy of power flows due to line impedances and errors in voltage measurements. On the other hand, the behavior of the microgrid will not be as optimum as it can be with centralized strategies and the complexity is increased compared with decentralized controls.

A very popular control strategy for microgrids is the hierarchical control [36]. This method separates the control objectives typically in three groups: primary, secondary, and tertiary controls. The primary level is in charge of the local control of the converters, they regulate their output voltage or current, using droop, MPPT, or current controls. These controls are the fastest, normally in time ranges under a second. The secondary control 
provides ancillary services to the microgrid, namely current sharing or power flow control of the sources within the microgrid, voltage restoration in case of using droop controllers, etc. These controllers are commonly more than one order of magnitude slower than the primary controllers, in the range of seconds. Finally, the tertiary level manages the power flow between the microgrid and the rest of the grid. This control can be based on optimization algorithms that take into account many variables as weather prediction, the price of the energy, user commands, etc. This is the slower control which can be in the range of minutes or hours.

In the literature, hierarchical strategies with distributed coordination are the most extended approach due to their suitability with smart grid applications. The trade-off between complexity and accuracy of distributed controllers is very interesting for these applications. Besides, the hierarchical control deals with the complexity of the system by dividing the control objectives in different layers, which work with different time ranges. Finally, optimization techniques can be easily integrated in such a way that they do not affect the reliability of the system, as in case of a failure in the communication network, the microgrid can still work in a non-optimum manner. A thorough review of control strategies for dc microgrids can be found in [37].

\section{$2.2 \quad$ Modeling electronic power converters}

Modeling EPCs is an essential part of the design process of the devices and their control structures. Besides, these models are also important to study dynamic interactions in interconnected systems and to perform stability analyzes. One of the contributions of this thesis is the development of an overview of modeling techniques, including analytical and blackbox approaches, focusing on their application to complex systems, such as dc microgrids [38]. Part of this work is described here.

Switching EPCs are essentially nonlinear time-variant systems. Nowadays, many electronic simulation tools are able to reproduce their behavior with high accuracy in time domain. However, the complexity of these models makes it difficult to use them for design or stability analysis purposes. In order to overcome this problem, very often, simplified models that represent this system with enough accuracy are used under certain assumptions. The most extended simplification method is the use of averaged models in order to eliminate the dependency on time. These nonlinear time-invariant models have been used for nonlinear stability analyzes or simulation purposes with reductions on the computational burden. Furthermore, often a linearization around an operating point is performed to the averaged models in order to obtain Linear Time-Invariant (LTI) models. This step allows the designer to use many relatively simple and powerful linear methods, such as Laplace transformation, bode plots, Nyquist stability criteria, etc.

\subsubsection{Averaging techniques}

The use of averaging techniques for EPCs to obtain time invariant models is widely extended. Different methods have been proposed in the literature able to account for different phenomena as ripple, effect of harmonics, discrete effects, etc. A review of the most common approaches is given here.

\section{State-space averaging}

State-Space Averaging (SSA) has been used very successfully in many applications during the last thirty years [39-44]. The idea is to describe the state-space equations in each of the switch configurations. Then the average value of the state variables in each switching cycle is obtained by weighting the different modes with the duty cycle. Afterwards, a per- 
turbation/linearization process can be performed to obtain the small-signal model.

If a Pulse Width Modulation (PWM) converter with duty cycles $d_{i}$ is considered, the average model would look as follows (2.2):

$$
\begin{aligned}
& \dot{x}=\sum_{i=1}^{n}\left[A_{i} d_{i} x+B_{i} d_{i} u\right] \\
& y=C x+D u
\end{aligned}
$$

where $x$ is the state vector, $u$ is the input vector, $y$ is the output vector, $\mathrm{A}$ is the state matrix, $\mathrm{B}$ is the input matrix, $\mathrm{C}$ is the output matrix and $\mathrm{D}$ is the feedthrough matrix.

This model will provide a very good estimation of the dynamic of the EPC if two conditions are complied with. First, the switching frequency should be much higher than the ones of interest and, second, the ripple of the state variables must be small enough. Also, a slow variation of the duty cycle is assumed. However, these conditions are very reasonable because the control stage will attenuate the high frequency signals and, in general, converters are designed to present a low enough ripple.

Nevertheless, there are some converters that, by concept, cannot be modeled with this technique. For instance, resonant converters do not comply with the first condition, because their time constants are of the same order as the switching frequency. On the other hand, Dual Active Bridge (DAB) converters controlled using phase-shift modulation do not meet the second condition, since they depend on state variables with zero average value in a switching cycle. DAB can be often found in microgrids designs associated with solid-state transformers $[45,46]$. Furthermore, in case a very high switching frequency is used, the side-band effect of close-loop converters becomes significant due to the PWM modulation, limiting the application of SSA.

\section{Generalized state-space averaging}

In order to model converters that do not comply with the SSA conditions, Generalized State-Space Averaging (GSSA) has been used [47, 48]. This method allows the analysis of variables with ac behavior or large ripple content. In this case, the averaging method is based on a time-dependent Fourier series representation for a sliding window. This method is frequency-selective since each element of the series represents the gain of the signal at each harmonic frequency. In fact, if only the dc term of the series is considered, the statespace averaging method is recovered. This method is based on the fact that the waveform $x(t)$ can be approximated with arbitrary precision in the $(t-T, t)$ range by the Fourier series [49], as represented in (2.3):

$$
x(t)=\sum_{k=-n}^{n}\langle x\rangle_{k}(t) e^{j k \omega t}
$$

where $\omega$ is the angular frequency, $t$ is time, and $\langle x\rangle_{k}(t)$ are the complex Fourier coefficients defined in (2.4):

$$
\begin{aligned}
w & =\frac{2 \pi}{T} \\
\langle x\rangle_{k}(t) & =\frac{1}{T} \int_{t-\tau}^{t} x(\tau) e^{-j k \omega \tau} d \tau
\end{aligned}
$$


where $T$ is the period. The selection of the value of $T$ will depend on the application, as switching period in case of PWM converters or fundamental period in general. The subscript $k$ refers to the index-k average, which corresponds to the $\mathrm{k}$-th harmonic. In order to recover the SSA representation only $k=0$ would be included, whereas $k=\{1,-1\}$ would account for the first harmonic content.

In order to obtain the state-space representation from the average variable from (2.4) two issues must be addressed related to the index-k average: differentiation with respect to time and computation of the product of two signals. The first issue is solved using Leibniz integral rule, the time derivative of each element of the series would have this form (2.5):

$$
\frac{d}{d t}\langle x\rangle_{k}(t)=\left\langle\frac{d}{d t} x\right\rangle_{k}(t)-j k w\langle x\rangle_{k}(t)
$$

where the first term of the right-hand side is usually approximated by a Describing Function (DF) (see Section 2.2.2), whereas the second issue can be computed using discrete convolution $(2.6)$ :

$$
\langle q x\rangle_{k}(t)=\sum_{i=-\infty}^{\infty}\langle q\rangle_{k-i}(t)\langle x\rangle_{i}(t)
$$

where $q$ is in general any other signal, but in case of converters it usually corresponds to the switching function. Further details about this modeling technique and illustrative examples can be found in [48]. This extended version of the previous method has been used, for example, to study resonant and DAB converters or to get ripple estimations for state variables $[47,50-52]$. This method has also been applied to system-level simulation of EPC architectures [49]. It must be considered that the complexity of this model grows considerably as the number of considered harmonics increases.

\section{Other averaging methods}

Even though the previous are the most extended averaging methods for EPCs, some alternative approaches have been proposed. Another common approach to consider higher order approximations is the Krylov-Bogoliubov-Mitropolsky (KBM) method, under the assumption of a small ratio between switching period and time constants of the system. The idea is to consider a geometric framework for the average model by means of a change of variables. Detailed information about this modeling approach can be found in [53-55]. More recently the Time-Invariant Multi-Frequency (TIMF) modeling technique was proposed based on the quasi-Fourier series representation. With this method it is possible to consider different kinds of carrier signals for PWM dc-dc converters [56]. Finally, the floquet-based or CyclicAveraging $(C A)$ method has also been proposed for rapid analysis and design of high order resonant EPCs, offering a ripple estimation in steady-state [57].

\section{Discrete averaged models}

In order to achieve more accurate results at high frequencies, higher than half of the switching frequency, analogue Discrete Averaged Method (DAM) have been proposed [58, 59]. These models are more complex, but they do not rely on frequency and ripple simplifications, thus they can be used to represent both resonant and DAB converters with phase-shift modulation mentioned before [60, 61]. Besides, when using digital control loops, discrete methods are able to account for sampling, modulation effect and delays [62], and systemlevel control impact on the stability [63]. Discrete-time methods have also been proposed for nonlinear stability analysis of dc distribution systems [64]. 
Table 2.2: Comparison of the capabilities of averaging techniques for dc EPCs. SSA (State-Space Averaging), DF (Describing Function), GSSA (Generalized State-Space Averaging), CA (Cyclic-Averaging), DAM (Discrete Averaged Model).

\begin{tabular}{|c|c|c|c|}
\hline Technique & Main advantages & Main drawbacks & $\begin{array}{l}\text { Typical applica- } \\
\text { tions }\end{array}$ \\
\hline SSA & $\begin{array}{l}\text { Simplicity. Good } \\
\text { performance. }\end{array}$ & $\begin{array}{l}\text { Small ratio switch- } \\
\text { ing period and time } \\
\text { constants. Small- } \\
\text { ripple. Slow varia- } \\
\text { tion of duty cycle. }\end{array}$ & Used broadly. \\
\hline GSSA & $\begin{array}{l}\text { Account for SSA lim- } \\
\text { itations. Multifre- } \\
\text { quency approach. }\end{array}$ & $\begin{array}{l}\text { Complexity increases } \\
\text { exponentially with } \\
\text { order. }\end{array}$ & $\begin{array}{l}\text { Ripple estimation. } \\
\text { DAB and resonant } \\
\text { converters. Ripple- } \\
\text { based controlled } \\
\text { converters. }\end{array}$ \\
\hline KMB & $\begin{array}{l}\text { Multifrequency } \\
\text { approach. Strong } \\
\text { mathematical frame- } \\
\text { work. }\end{array}$ & $\begin{array}{l}\text { Small ratio switching } \\
\text { period and time con- } \\
\text { stants. Complexity. }\end{array}$ & $\begin{array}{l}\text { Ripple estimation. } \\
\text { DAB and resonant } \\
\text { converters. Ripple- } \\
\text { based controlled } \\
\text { converters. }\end{array}$ \\
\hline TIMF & $\begin{array}{l}\text { Multifrequency } \\
\text { approach. System- } \\
\text { atic derivation from } \\
\text { SSA. }\end{array}$ & $\begin{array}{l}\text { Slow variation of } \\
\text { duty cycle assumed. } \\
\text { Moderate complex- } \\
\text { ity. }\end{array}$ & $\begin{array}{l}\text { Different kinds of } \\
\text { carriers signals. } \\
\text { Ripple estimation. }\end{array}$ \\
\hline $\mathrm{CA}$ & $\begin{array}{l}\text { Fast and accu- } \\
\text { rate steady-state } \\
\text { information. }\end{array}$ & $\begin{array}{l}\text { Absence of dynamic } \\
\text { information. Com- } \\
\text { plexity. }\end{array}$ & $\begin{array}{l}\text { Rapid analysis of } \\
\text { high order resonant } \\
\text { converters. Rip- } \\
\text { ple estimation in } \\
\text { steady-state. }\end{array}$ \\
\hline DAM & $\begin{array}{l}\text { Account for dis- } \\
\text { cretization effects. }\end{array}$ & Complexity. & $\begin{array}{l}\text { High frequency. Rip- } \\
\text { ple. Sampling, mod- } \\
\text { ulation, delays ef- } \\
\text { fects, etc. }\end{array}$ \\
\hline
\end{tabular}

In summary SSA is the most widely used method, because of its simplicity and good performance for the applications that comply with its conditions. In case higher order approximations are required, GSSA or KBM methods are normally applied. These two strategies are very similar conceptually, which are based on the segregation of the response of the variables in their harmonic contributions. However the implementation of this idea is completely different. GSSA is based on Fourier series, whereas KBM is based on a change of variables that creates a geometric framework. A comparison between these two approaches can be found in [65]. Furthermore, other methods have also been proposed to solve some problems as considering different types of carrier signals (TIMF), rapid analysis of steady-state behavior (CA), or considering digital effects (DAM). A comparison among these methods can be found in [56]. In Table 2.2 the main advantages, drawbacks, and applications of the different averaging methods described are summarized.

Focusing on smart grid applications, particularly on dc micro/nanogrids, there are many works related to individual EPCs that solve some of the challenges involved in these systems. However, it is also very important to analyze the phenomena that arise due to the 
interconnection of many EPCs, the intermittent behavior of both renewable sources and house loads, bidirectional power flow of batteries and grid connected converters, etc. In this context most of the proposals use the state-space averaging strategy, selecting the most common operating points in the system for modeling purposes, or the worst case scenario for small-signal stability assessment [66-72].

Clearly, the small-signal approximation implies a limitation in the modeling strategy, especially when the power converters are working in the boundary of two operation modes. In these cases the prediction of the small-signal models are seriously compromised. It is like the classical problem of $\mathrm{dc} / \mathrm{dc}$ converters working close to the boundary between continuous and discontinuous modes.

In case of multiple EPCs, some other works consider the effect of the different switching frequencies of the EPCs using the extended state-space averaging technique, which is a modification of the generalized state-space averaging method in order to consider the harmonics of more than one switching frequency [73]. The large-signal behavior is analyzed with the nonlinear model, but the model is linearized around an operating point in order to perform the small-signal stability assessment. In [74] a stabilization method is proposed for decentralized distribution systems, using discrete-time SSA models of the converters and applying neural networks to deal with nonlinearities and unknown dynamics. Therein a review of system-level stabilization techniques can be found. Finally, in [75] SSA is used to perform nonlinear stability analysis of dc microgrids by means of the computational continuation. Stability boundaries related with some of the parameters of the system are obtained with this method.

\subsubsection{Modular and nonlinear analytical modeling techniques}

DC microgrids are composed of several elements interconnected together in a specific architecture. In order to cope with large-scale systems, dozens of converters working in the same microgrid, some modular linear and nonlinear techniques have been proposed. Besides, due to system control strategies both static and dynamic nonlinearities must be taken into account, for which quasi-linear or multi-model techniques can be found. Quasi-linear approximations consider linear representation depending on some of the properties of the input signal, whereas multi-model approaches attempt to capture the nonlinear behavior of a system defining different representations of its response. Then, the output of the model will be function of certain variables that will activate some of the multiple representations.

\section{Two-port model}

The two-port representation is very suitable for interaction assessments in EPC based systems, details about this method can be found in [76]. The main advantage of this technique is that very large systems can be divided into subsystems, which can be easily combined shaping any desired architecture. In order to build these blocks, the idea is to obtain the parameters that characterize the input-output small-signal dynamical behavior of each subsystem. In case of voltage-controlled EPC connected to a voltage source, the inverse hybrid parameters (G-parameters) model is usually used, obtaining a Norton and Thevenin equivalent circuit. Therefore, the representation of the model has the form:

$$
\left(\begin{array}{c}
v_{o} \\
i_{\text {in }}
\end{array}\right)=\left(\begin{array}{cc}
G_{21}(s) & -Z_{o}(s) \\
Y_{i}(s) & H_{12}(s)
\end{array}\right)\left(\begin{array}{c}
v_{i n} \\
i_{o}
\end{array}\right)
$$

where $u$ and $i$ are voltage and current, the subscripts $o$ and in indicate output and input respectively. These transfer functions can be obtained from any of the averaged representations described in the previous section, after a linearization process around a particular operating point. The physical meaning of the G-parameters is: 
Audio-susceptibility Input admittance

$G_{21}(s)=\left.\frac{\tilde{v}_{\text {out }}}{\tilde{v}_{\text {in }}}\right|_{\tilde{i}_{\text {out }}=0}$

Output impedance

$$
Z_{o}(s)=\left.\frac{\tilde{v}_{\text {out }}}{\tilde{i}_{\text {out }}}\right|_{\tilde{v}_{\text {in }}=0}
$$

$$
Y_{i}(s)=\left.\frac{\tilde{i}_{i n}}{\tilde{v}_{\text {in }}}\right|_{\tilde{i}_{\text {out }}=0}
$$

Back current gain

$$
H_{12}(s)=\left.\frac{\tilde{i}_{\text {in }}}{\tilde{i}_{\text {out }}}\right|_{\tilde{v}_{\text {in }}=0}
$$

Notice that the linearization step is needed for this modeling approach because it is based on the superposition theorem, i.e. the output variables can be defined as the independent contribution of each of the input variables when the other inputs are set to zero. This method has been widely used for the analysis of supply and load interactions, as well as for system-level impedance-based stability assessment [77-82].

Some other representations of two-port models are possible by modifying the choice of input and output variables. Usually, the variable controlled by the input source is set as an input and the variable controlled by the converter, in case of a regulated EPC, is set as an output. Consequently, the remaining output variable is set as an input, because is commonly determined by the regulated variable and the load. Finally, the last input variable is set as the other output.

Using the voltages of the ports as outputs and the currents as inputs the impedance parameters (Z-parameters) model is obtained:

$$
\left(\begin{array}{c}
v_{i n} \\
v_{o}
\end{array}\right)=\left(\begin{array}{cc}
Z_{11}(s) & Z_{12}(s) \\
Z_{21}(s) & Z_{22}(s)
\end{array}\right)\left(\begin{array}{c}
i_{i n} \\
i_{o}
\end{array}\right)
$$

This model would be used for a voltage-controlled converter connected to a current source. An advantage of this model is that the series connection of Z-parameters models is just the sum of each of the matrices.

Equivalently, using the currents of the ports as outputs and the voltages as inputs the admittance parameters (Y-parameters) model is obtained:

$$
\left(\begin{array}{c}
i_{i n} \\
i_{o}
\end{array}\right)=\left(\begin{array}{cc}
Y_{11}(s) & Y_{12}(s) \\
Y_{21}(s) & Y_{22}(s)
\end{array}\right)\left(\begin{array}{c}
v_{i n} \\
v_{o}
\end{array}\right)
$$

This model would be used for a current-controlled converter connected to a voltage source. Similarly, the parallel connection of Y-parameters model is just the sum of each of the matrices.

Finally, the hybrid parameters (H-parameters) model sets the input voltage and output current as outputs of the model, whereas the input current and the output voltage are the inputs:

$$
\left(\begin{array}{c}
V_{i n} \\
i_{o}
\end{array}\right)=\left(\begin{array}{cc}
Z_{i}(s) & G_{12}(s) \\
H_{21}(s) & Y_{o}(s)
\end{array}\right)\left(\begin{array}{c}
i_{i n} \\
v_{o}
\end{array}\right)
$$

This model would be used for a current-controlled converter connected to a current source. 
Taking advantage of the superposition theorem, many authors have extended these models to include the effect of other variables. The most common choice is to include the effect of the duty cycle on the output variables. Using the G-parameters model as a base, the new model including the effect of the duty cycle (d), assuming that only one duty cycle defines the average model of the converter, would be:

$$
\left(\begin{array}{c}
v_{o} \\
i_{\text {in }}
\end{array}\right)=\left(\begin{array}{ccc}
G_{21}(s) & -Z_{o}(s) & G_{c v}(s) \\
Y_{i}(s) & H_{12}(s) & G_{c i}(s)
\end{array}\right)\left(\begin{array}{c}
v_{\text {in }} \\
i_{o} \\
d
\end{array}\right)
$$

Furthermore, from this representation it is possible to represent the G-parameters model of the regulated EPC as a function of the open loop G-parameters, the transfer functions from the duty cycle and the output variables, and the control parameters. This model can be obtained by just expressing the duty cycle as the control multiplied by the error between the reference and the controlled variable.

\section{Component connection model}

The analysis of large systems is a multidisciplinary classical problem. Long ago, the component connection model was proposed as a methodology to interconnect the mathematical representation of different individual elements to obtain the system-level expression, for instance to perform nonlinear stability analysis. This method is basically a mapping among the inputs and outputs of the components that form the system. The matrix equation can be represented as follows (2.13):

$$
\left(\begin{array}{l}
a \\
y
\end{array}\right)=\left(\begin{array}{ll}
L_{11} & L_{12} \\
L_{21} & L_{22}
\end{array}\right)\left(\begin{array}{l}
b \\
u
\end{array}\right)
$$

where $b$ and $u$ are the vectors of inputs of the elements and the system, and $a$ and $y$ are the vectors of outputs of the elements and the system respectively. This is a very useful method to form large-scale systems with a modular approach. A review of this method with a collection of references can be found in Chapter 7 of [83]. This method is applicable with any level of abstraction, for instance, in [84] it was used for EPC based systems, whereas in [85] it was used at EPC level in order to obtain an efficient and detailed model. This method seems very suitable for dc microgrids, however, references using this approach have not been found.

\section{Describing function}

A classical method to take into account some nonlinear effects in EPCs is to use the DF; a comprehensive coverage of this method can be found in [86]. Using this technique it is possible to determine limit cycles and the dynamic behavior of nonlinear systems.

This method is based on the quasi-linearization of the nonlinearity, i.e. to obtain a linear model except that it depends on some of the properties of the input signal. In order to apply this technique, the type of input signal is commonly specified and the nonlinear behavior is considered to be only dependent on the amplitude of the input signal. Thus the describing function method is often preceded by the kind of input considered. As most of the systems have a filtering effect, the most common input signals studied are: bias, sinusoid, Gaussian process, or a linear combination of them. The most extended method is to consider the sinusoidal-input describing function, often found just as describing function.

This technique considers a linearized feedback loop, (Fig. 2.5), where it is possible to divide the system into a linear, $G(j \omega)$ and a nonlinear part, where the nonlinear part is 


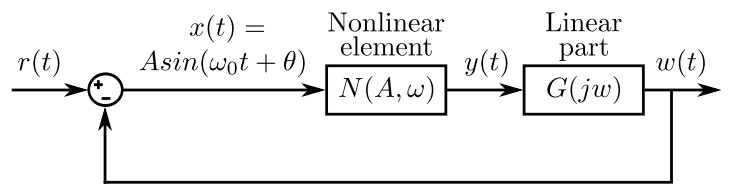

Figure 2.5: Feedback system with a nonlinear element represented by a describing function.

represented by the describing function $N(A, \omega)$, that depends on the amplitude, $A$, of its input signal, $x(t)$, and the frequency, $\omega$. The feedback loop has a reference, $r(t)$, and an output, $w(t)$ that would depend on the rearrangement considered. The describing function is usually obtained using a Fourier first order approximation, reducing to $N(A)$. Using this representation the condition for a steady-state oscillation, considering a reference $r(t)=0$, is $(2.14)$ :

$$
1+G(j \omega) N(A)=0
$$

or equivalently (2.15):

$$
G(j \omega)=\frac{-1}{N(A)}
$$

This method offers the possibility to represent graphically these functions to find approximations of limit cycles, which will be defined by the intersection between those curves, $\left(x_{o}, w_{o}\right)$.

The assumptions considered in this approximation are that only one single nonlinear component exists, that it is time invariant, and that is has low-pass filter properties. The last condition is known as the filter hypothesis, which is necessary to ensure a sinusoidal input to the nonlinear part. Typical examples of these kinds of nonlinearities are saturated actuator, hysteresis in magnetic materials, or dead zones.

In the case of EPCs it has been used to represent the nonlinear behavior of inductors so continuous and discontinuous conduction mode behaviors can be estimated in [87] or to consider the nonlinearities of the switching process in [88].

\section{Hybrid modeling}

In essence EPCs are hybrid structures composed of a number of continuous dynamical systems controlled by discrete restrictions or commands. Consequently if a very detailed model is required, this hybrid nature must be taken into account. Hybrid systems theory was developed by Branicky et al. in the nineties, although preliminary studies were published many years before. In [89] a thorough explanation of this kind of system and a review of previous related works can be found. This modeling approach considers both continuous and discontinuous variables. The system dynamic depends on the evolution of the system equations, which are generally function of both types of variables.

Basically, the hybrid model represents a collection of continuous models combined with a switching function able to jump from one model to another when the state variables reach certain inherent values or when an external control signal is activated. These large-signal hybrid models may be summarized in the following equations (2.16):

$$
\begin{aligned}
\dot{x}(t) & =f(x(t), q(t), u(t)) \\
q^{+}(t) & =v(x(t), q(t), u(t))
\end{aligned}
$$


where $x(t)$ is the system state vector, $q(t)$ is the discrete state vector, $q^{+}(t)$ is the next value of the discrete state vector, and $u(t)$ is the input vector. Through the $q(t)$ vector the different continuous models are switched as a function of certain inherent conditions defined through certain values of the state variables $x(t)$, or through the inputs $u(t)$.

This modeling technique has been applied in several different fields. In power electronics it is particularly relevant when multiple operation modes or saturation effects of the EPC must be taken into account. Within the hybrid modeling framework, different classes of representations can be considered. In [90] some classes of hybrid systems for EPC are reviewed and a Piece-Wise Affine (PWA) model is used for controller design and nonlinear stability analysis of a resonant converter. PWA models are switched affine systems whose modes only depend on the current location of the state vector. The state-space is partitioned into a finite number of polytopic cells, constructed as the intersection of half spaces. This technique provides the interesting possibility of mixing linear and nonlinear methods.

In [91, 92] hybrid modeling was used to develop predictive control strategies. Also Lyapunov stability analyses have been employed in order to assure global stability under every possible condition.

\section{Linear parameter-varying model}

A different way to represent nonlinear systems is by using the Linear Parameter-Varying (LPV) models. These are linear state-space representations which dynamics depend on time-varying parameters. This method has been used extensively in many applications for controller design and stability analysis of nonlinear plants. In general, the mathematical representation can be expressed as follows (2.17):

$$
\begin{aligned}
& \dot{x}=A\left(p_{i}\right) x+B\left(p_{i}\right) u \\
& y=C\left(p_{i}\right) x+D\left(p_{i}\right) u
\end{aligned}
$$

where $p_{i}$ are the variables affecting the dynamic of the system. Different methods can be used in order to represent the varying dynamics of the system, a thorough review of LPV models and its different method can be found in [93].

One of these methods is the polytopic model. As in the case of the PWA model, this is a multi-model approach in which a collection of linear models are obtained around different operating points, however instead of switching among linear models, in this case the overall response is a weighted combination of the neighboring linear models. The state-space equations of this model are represented as follows (2.18):

$$
\begin{aligned}
& \dot{x}=\sum_{i=1}^{n} \omega_{i}(\alpha, \beta, \ldots)\left(A_{i} x+B_{i} u\right) \\
& y=\sum_{i=1}^{n} \omega_{i}(\alpha, \beta, \ldots)\left(C_{i} x+D_{i} u\right)
\end{aligned}
$$

where $\omega_{i}$ are the weighting functions of the linear models and $\alpha$ and $\beta$ are the variables considered in the polytopic model as the ones that most affect the behavior of the system. This technique has been widely used in several fields. In [94] the stability of nonlinear systems is studied by means of Lyapunov methods applied to polytopic models solving Linear Matrix Inequality (LMI) problems. Also in $[84,95]$ polytopic models were proposed in order to obtain Lyapunov candidates for stability analysis of distributed power systems. Furthermore, different techniques are also based in this modeling approach as Takagi-Sugeno fuzzy logic models, where the weighting functions are obtained based on a number of if/then rules 
Table 2.3: Comparison of the capabilities of modular and nonlinear techniques for dc EPC systems. GP (G-parameters), CC (Component Connection), HM (Hybrid Modeling), LPV (Linear Parameter-Varying).

\begin{tabular}{|c|c|c|c|}
\hline Technique & Main advantages & Main drawbacks & $\begin{array}{l}\text { Typical applica- } \\
\text { tions }\end{array}$ \\
\hline GP & $\begin{array}{l}\text { Modularity. Simplic- } \\
\text { ity. Good perfor- } \\
\text { mance. }\end{array}$ & Small-signal. & $\begin{array}{lr}\text { Dynamic } & \text { interac- } \\
\text { tions. } & \text { Impedance- } \\
\text { based } & \text { stability } \\
\text { analysis. } & \end{array}$ \\
\hline $\mathrm{CC}$ & $\begin{array}{lr}\text { Modular } & \text { approach } \\
\text { for } & \text { state-space } \\
\text { models. } & \end{array}$ & $\begin{array}{l}\text { Just a mapping tech- } \\
\text { nique. }\end{array}$ & $\begin{array}{l}\text { Nonlinear stabil- } \\
\text { ity analysis. Very } \\
\text { detailed models. }\end{array}$ \\
\hline DF & $\begin{array}{l}\text { Analysis of some } \\
\text { nonlinear effects. }\end{array}$ & $\begin{array}{l}\text { Single nonlinearity. } \\
\text { Filter hypothesis. }\end{array}$ & $\begin{array}{l}\text { Limit cycles. Stabil- } \\
\text { ity analysis. Non- } \\
\text { linearities in switch- } \\
\text { ing process, magnet- } \\
\text { ics, etc. }\end{array}$ \\
\hline $\mathrm{HM}$ & $\begin{array}{l}\text { Continuous and dis- } \\
\text { continuous variables. } \\
\text { Accuracy. }\end{array}$ & $\begin{array}{l}\text { Complexity can be } \\
\text { very high. }\end{array}$ & Used broadly. \\
\hline LPV & Large-signal. & $\begin{array}{l}\text { Complexity. } \\
\text { nonlinearities. }\end{array}$ & $\begin{array}{l}\text { Nonlinear stabil- } \\
\text { ity analysis and } \\
\text { controller design. }\end{array}$ \\
\hline
\end{tabular}

[96-98] or neural networks, where the system learns from input/output data to be able to tune the model $[99,100]$.

The previous techniques seem very suitable for dc microgrids. G-parameters models are very convenient for large-scale small-signal simulations and dynamic interaction and stability analyses, because of its modular approach. For nonlinear assessments, DF, hybrid or LPV models can be employed. Very detailed low level models, as those obtained using hybrid models, are very useful for simulation purposes, but a simpler option is needed for stability analysis. For this or to obtain computationally efficient representations, DF or polytopic LPV models are a possibility. Besides, the component connection model technique can be used in order to obtain the overall system-level equations of the system from a modular perspective.

\subsection{Blackbox modeling structures}

In actual applications, very often smart microgrids are implemented using devices from different manufacturers and the information given to the users is very limited to have a detailed model of the system dynamics. Having these limitations in mind, it is valuable to develop a model, accurate enough, to foresee the dynamics of the generators, loads, and storage units, ensuring the power quality and the stability of the system. If the manufacturers do not provide detailed and accurate information about the dynamic of the converters, the solution must come from the application of identification techniques to the EPCs.

In general, system identification is a very complex task. For linear systems there are several well-established techniques which are used broadly, whereas the case of nonlinear 
systems is much more difficult. Functional series (Volterra or Wiener series) were proposed as a general structure that can be applied to a wide class of systems. They are very useful to represent mild nonlinearities, however the complexity of the model increases exponentially with the order considered.

A common approach to efficiently consider harsh nonlinearities is to include some knowledge about the system to select a more suitable structure for the blackbox model. Depending on the application and the level of knowledge about the system, it is possible to approximately write the state-space equations with unknown parameters that will be estimated afterwards. This method has been called greybox modeling.

If there is not much information about the system, another option is to use Wiener and Hammerstein structures. This approach separates the response in two parts: one linear to represent the dynamic of the system and one nonlinear that represents the static nonlinearities of the system.

In case that the nonlinearities affect also the dynamics of the system, more complex solutions have been proposed, as polytopic models. This technique consists in an interpolation of the response of linear models obtained in different operating points, as it was described in Section 2.2.2 for analytical models.

Considering dc microgrids using DBS control, it is possible to find nonlinearities that are part of the system operation. These nonlinearities can be static, as the steady-state V-I relations shown in Fig. 2.4 for the different EPCs, or dynamic, such as the transient behavior of the converters in the different operation modes.

\subsubsection{Small-signal structure}

The analytical two-port models have blackbox counterparts. For the sake of simplicity the discussion will be based on the G-parameters model, however it is analogous for any other two-port representations. Here the linear models are obtained from measurements at the external ports of the EPC. In general, as the control signal is not usually available, the effect of the controller is mixed with the behavior of the plant, so an overall input-output response is obtained from the measurements (Fig. 2.6). An advantage of this modeling approach is that as a Thevenin/Norton representation of the converter is obtained, some physical insight is acquired (see (2.8)).

The methodology to obtain the G-parameters for a converter working in a stationary operating point can be found in $[101,102]$. Due to its relevance in this thesis, the procedure is briefly described here. The approach involves the use of a voltage source and a controllable current load in order to set a fix operating point. Notice that in order to apply superposition, keeping a variable constant is equivalent to set its small-signal contribution to zero. Two tests are needed to obtain the four G-parameters (Fig. 2.7). In the first test a perturbation is introduced in the load current, while keeping the input voltage constant, and the response of the output variables is measured. From this test the output impedance and the back current gain can be identified. The second test is analogous, introducing the perturbation in the input voltage in this case, while keeping the output current constant. From this test the audiosusceptibility and the input admittance can be identified.

In general the output impedance of the sources and the input admittance of controllable loads is designed to be small so they do not affect the rest of the system. However, it is possible that in some applications their effect is not negligible. In [102] the methodology to obtain unterminated models is presented, i.e. a model that is not affected by the impedances of the source and the load used to perform the tests. The approach is to determine the contribution of these impedances in the response of the system and remove them from the final model. 
(a)

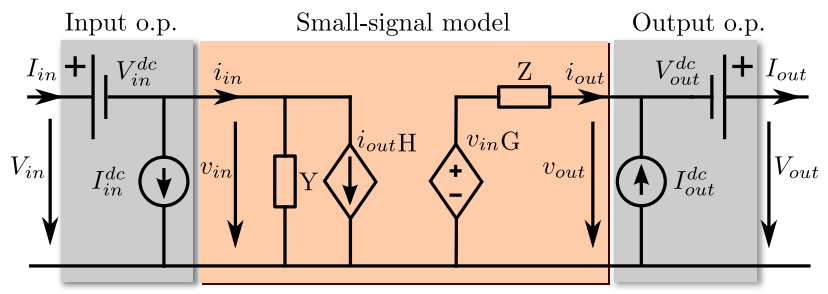

(b)

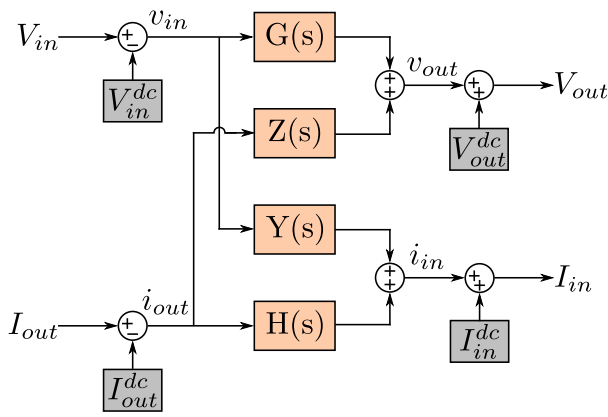

Figure 2.6: G-parameters model. (a) Equivalent electrical circuit, (b) Block diagram notation.

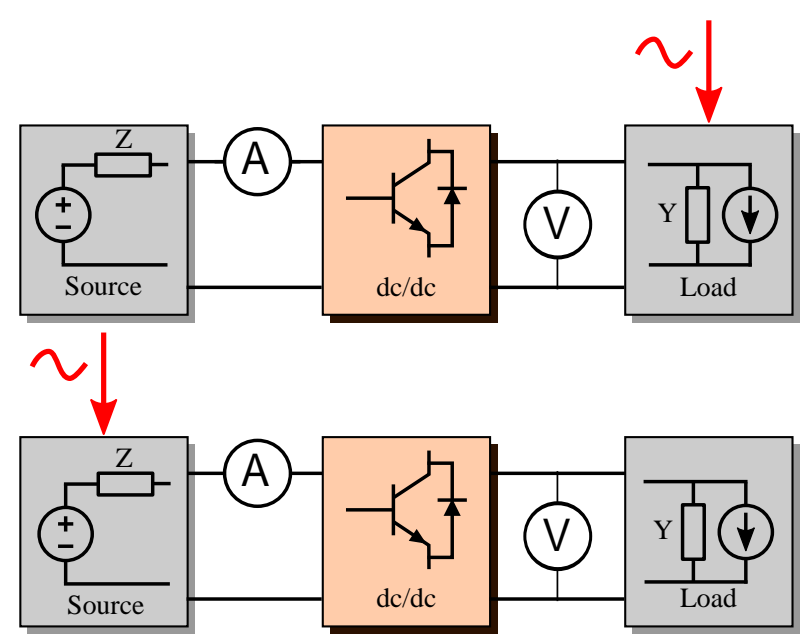

Figure 2.7: Test procedure to identify the G-parameters model. (a) Load perturbation , (b) Source perturbation. 
(a)

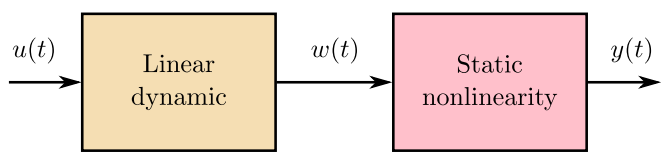

(b)

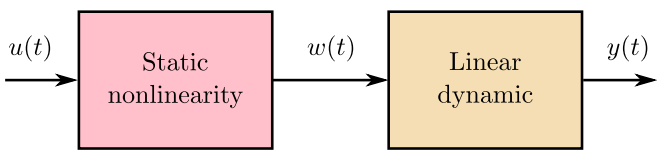

Figure 2.8: (a) Wiener model, (b) Hammerstein model.

This technique has also been applied to electronic power distribution systems based on COTS converters [103]. Having in mind the limitations of the small-signal assumption, this model offers a good representation of the dynamic of EPCs.

\subsubsection{Large-signal structures}

\section{Static nonlinear structure}

Some of the most popular blackbox nonlinear techniques are based on the Wiener and Hammerstein modeling strategies, which are valid when the nonlinearities are mainly reflected in steady-state variables. It is not unusual that while the dynamics of the system can be approximated by linear networks, static nonlinear effects appear due to saturations, nonlinear behavior of actuators or sensor, etc.

In Fig. 2.8, the block diagrams of the Wiener and Hammerstein models are represented. In the Wiener approach, a linear transfer function representing the system dynamics is followed by a nonlinear block, representing the steady-state operating point. In the Hammerstein model the structure is the opposite, having a nonlinear steady-state block followed by the linear block, able to foresee the system dynamics. Making the corresponding measurements, as indicated in [104], MATLAB/SIMULINK is able to identify the corresponding transfer functions of either Weiner or Hammerstein models.

There is a third possibility identified as Hammerstein-Wiener model, represented in Fig. 2.9a, where the system is represented by three blocks. The block in the middle is representing the linear system dynamics, and the stationary nonlinearities are represented by one block at the input and one block at the output. This structure is typically used when there are nonlinear physical conversions, e.g. nonlinear response of sensors or actuators. The identification of the corresponding transfer functions is also provided by MATLAB/SIMULINK, through data obtained with proper experimental measurements, as indicated in [104].

There are some other alternatives based on the same concept. As an example Oliver et al. proposed a model with a block diagram represented in Fig. 2.9b. In this model, especially developed for $\mathrm{dc} / \mathrm{dc}$ converters, the steady-state nonlinear behavior is included in a specific nonlinear block in the middle. The system dynamics are divided in three passive networks, one at the input, modeling some of the input effects like soft and hard start and Electro-Magnetic Interference (EMI) filter; one at the output representing the dynamics of the output filter and the control loops; and one from the input to the output, accounting for the audiosusceptibility (Fig. 2.10). As described in this work [105], just with the common information included in the datasheets or with very simple tests, a very simple 
(a)

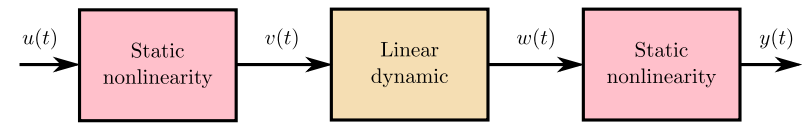

(b)

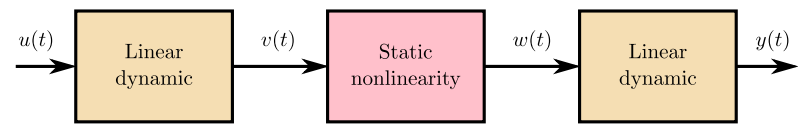

Figure 2.9: (a) Hammerstein-Wiener structure, (b) Wiener-Hammerstein structure.

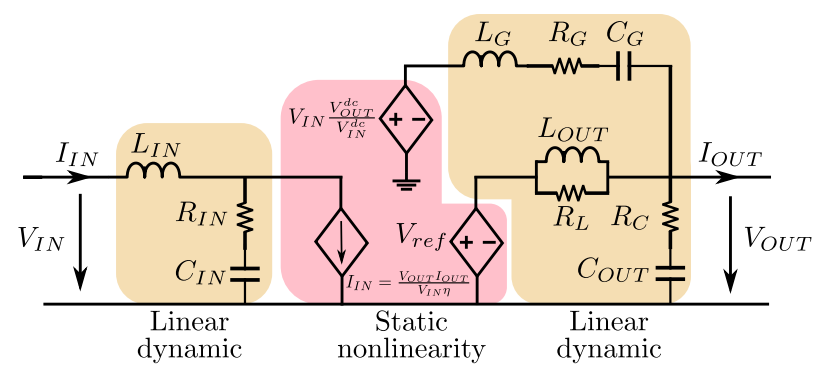

Figure 2.10: Oliver's Wiener-Hammerstein model.

and quite accurate model of a general dc EPC can be obtained.

\section{Dynamic nonlinear structure}

Finally, also the polytopic model (2.2.2) has an analogue blackbox counterpart. In this case, small-signal G-parameters models are used as the linearized model around different operating points. Therefore, compared with Wiener-Hammerstein structures, this model is able to capture the nonlinearities not only affecting the stationary, but also the transient behaviors. On the other hand, the complexity of this technique increases with the number of operating points considered and it grows exponentially with the number of variables taken into account. So a tradeoff between accuracy and complexity has to be made.

The main concept is represented in Fig. 2.11, where the output of the model is a weighted combination of the output of several LTI models obtained in different operating points. The weighting functions can be nonlinear trying to capture the large-signal behavior of the converter. A wide range of weighting or scheduling functions are suitable for this purpose, from the straightforward triangular function used in Takagi-Sugeno models [96] to more elaborated nonlinear alternatives as double sigmoid functions, which can be defined as follows:

$$
\begin{aligned}
\omega_{i}(\alpha, \beta, \ldots)= & \left(\frac{1}{1+e^{v_{\alpha i}(\alpha)}}-\frac{1}{1+e^{v_{\alpha i+1}(\alpha)}}\right) . \\
& \cdot\left(\frac{1}{1+e^{v_{\beta i}(\beta)}}-\frac{1}{1+e^{v_{\beta i+1}(\beta)}}\right) .
\end{aligned}
$$

with 


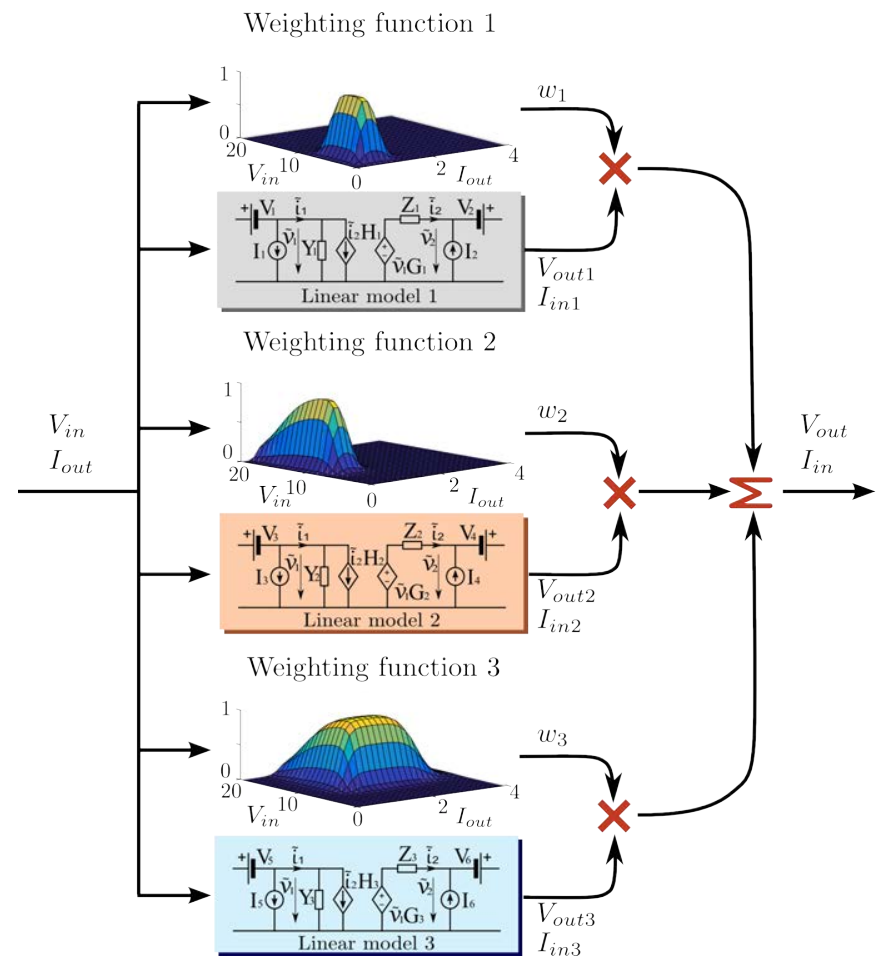

Figure 2.11: Polytopic model structure.

$$
v_{x i}(x)=-m_{x i}\left(x-c_{x i}\right)
$$

where $m_{x i}$ and $c_{x i}$ are the slope and the center of the i-th sigmoid of the variable x, respectively. This definition enables the development of complex multidimensional functions, while the weighting function constrains are assured:

$$
\begin{gathered}
0 \leq \omega_{i}(\alpha, \beta, \ldots) \leq 1 \\
\sum_{i=1}^{n} \omega_{i}(\alpha, \beta, \ldots)=1
\end{gathered}
$$

Furthermore, these two new parameters offer two variables that can be adjusted to improve the transition among small-signal models. Finally, these parameters are suitable to be optimized for a given objective function, e.g. using minimum error.

This kind of model shows a number of degrees of freedom that are crucial in terms of the model complexity and accuracy. In particular, three are the key choices: the number of variables considered, the number and location of the operating points selected, and the type of weighting function. A detailed explanation of the development of blackbox polytopic models for EPCs can be found in [106].

\section{Other blackbox models}

Some authors have proposed modeling structures which are a combination of the previous blackbox models. For instance in [107] a combination of G-parameters and WienerHammerstein models is proposed. It represents the dynamic behavior of the converter with a G-parameters model, but steady-state values are given by a static nonlinear block. 
Another possibility, proposed in [108], is to use a combination of all three methods depending on the necessities of each converter. For instance in that paper it is proposed to use G-parameters for input admittance and output impedance, the back current gain transfer function with a static behavior given by power balance, and the polytopic model for the audiosusceptibility. The main purpose of this proposal is to achieve a good compromise between accuracy and complexity for each converter.

A comparison among the different blackbox modeling approaches is presented in Chapter 3 .

\subsection{Dynamic interaction and stability analysis of dc-distributed systems}

Traditionally, stability analysis of EPCs-based power distribution systems has been based on impedance-based criteria. These methods rely on the small-signal condition, meaning that the converters are linear or they work close to a specific operating point. This approach is relatively simple and very powerful, as it can also give information about the margin of stability of the interconnected system. However, due to the variability in dc microgrids and the presence of Constant Power Load (CPL)s, the small-signal approximation cannot be always applied. Therefore, it is interesting to combine this linear analysis with large-signal approaches, which provide information about the Region Of Attraction (ROA) of nonlinear systems around an equilibrium point.

\subsubsection{Impedance-based stability criteria}

The impedance-based stability criteria usually consider a system consisting of a source and a load. Besides, it is assumed that this system is built with converters which are stable in standalone operations, which is a very reasonable assumption for a COTS convertersbased microgrid. In this scenario, the small-signal stability of the series-connected system, around an operating point, is determined by the output and input impedances of the source and load converters respectively. This idea is illustrated by considering the G-parameters models (see (2.7)) of the source and load converters and deriving the series-connected Gparameters model from this information. This derivation is included in Appendix A, which results in:

$$
\left(\begin{array}{c}
v_{o}^{2} \\
i_{i n}^{1}
\end{array}\right)=\left(\begin{array}{cc}
\frac{G_{1} G_{2}}{1+Z_{1} Y_{2}} & -\frac{Z_{2}+Z_{1}\left(Y_{2} Z_{2}+H_{2} G_{2}\right)}{1+Z_{1} Y_{2}} \\
\frac{Y_{1}+Y_{2}\left(Y_{1} Z_{1}+H_{1} G_{1}\right)}{1+Z_{1} Y_{2}} & \frac{H_{1} H_{2}}{1+Z_{1} Y_{2}}
\end{array}\right)\left(\begin{array}{c}
v_{i n}^{1} \\
i_{o}^{2}
\end{array}\right)
$$

From this expression it can be deduced that, provided that the converters are independently stable (their G-parameters are stable transfer functions), the stability of the interconnected system depends on the minor loop gain $Z_{1} Y_{2}$. Therefore, applying the Nyquist criterion to the minor loop gain a necessary and sufficient condition of stability for the interconnected system can be obtained. Based on this analysis several robust stability criteria have been proposed. The approach is to define a forbidden region in the s-plane, such that if the minor loop gain does not enter this region, the system will be stable with a desired margin of stability. 


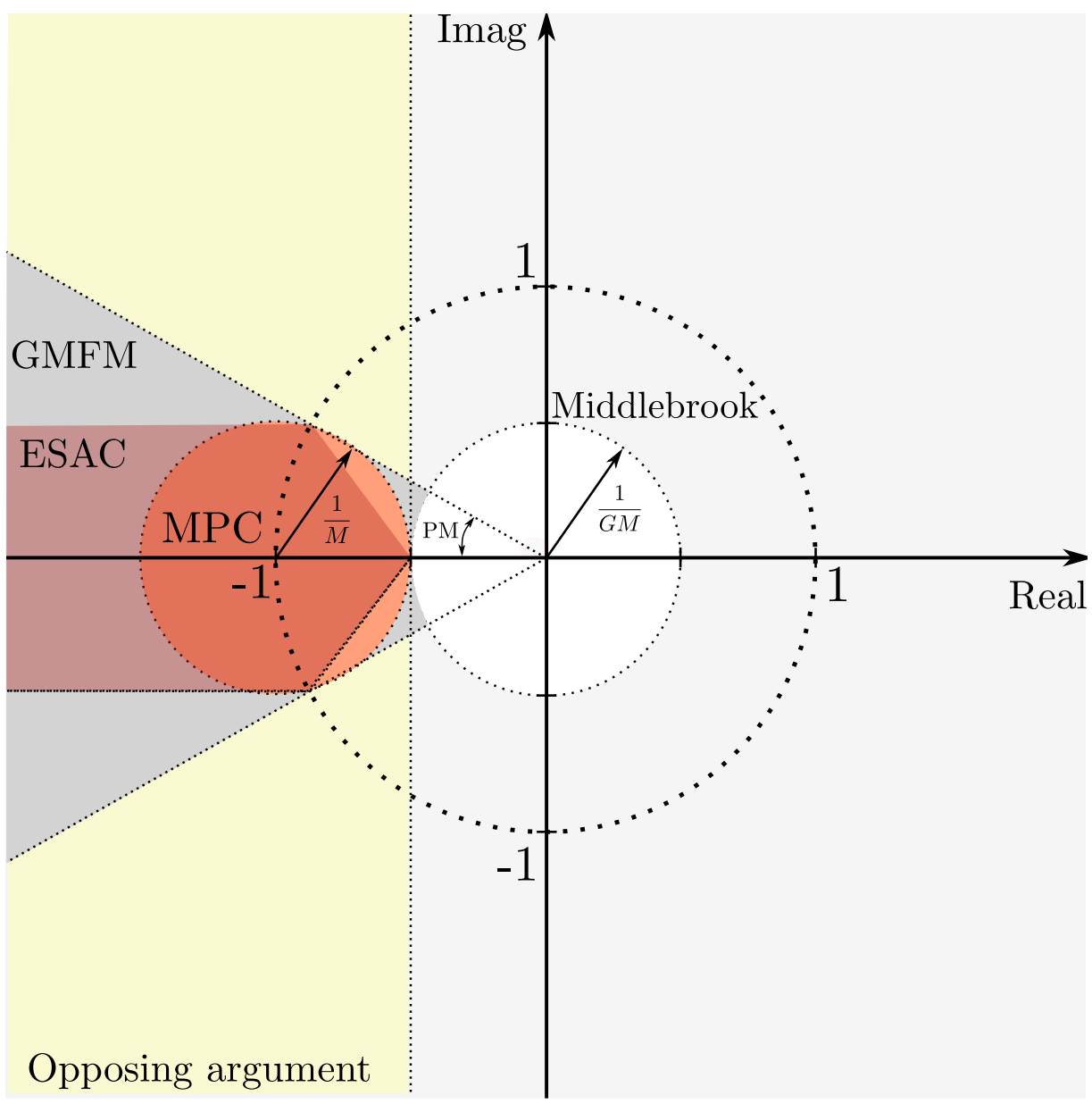

Figure 2.12: Forbidden regions.

\section{Middlebrook criterion}

The Middlebrook criterion originally studied how a controlled EPC is affected by the addition of an input filter. Afterwards, this method was generalized for any cascaded system that can be divided in a source and a load. The Middlebrook condition for the minor loop gain is:

$$
\left\|Z_{1} Y_{2}\right\| \ll 1
$$

Usually, the limit is set to $\frac{1}{2}$ or equivalently $-6 \mathrm{~dB}$. In the polar plot, this means that the minor loop should stay inside a circle with a radius equal to this value. This is the most restrictive criterion, but it also limits strongly the dynamic interactions between the converters, as it can be deduced from (2.22).

\section{Gain margin and phase margin criterion}

The Gain Margin Phase Margin (GMPM) proposes more relaxed conditions for stability in order to reduce the conservativeness of the Middlebrook criterion. This method not only considers a condition for the gain of the minor loop, but also takes into account the phase. The condition is: 


$$
\left\|Z_{1} Y_{2}\right\| \leq \frac{1}{G M} \text { and }\left|\arg \left(Z_{1}\right)+\arg \left(Y_{2}\right)\right| \leq 180-P M
$$

where $G M$ is the gain margin and $P M$ is the phase margin. Compared with the Middlebrook criterion, it allows the minor loop gain to violate the gain margin condition in case the phase margin is complied with. In other words, the Middlebrook criterion is equivalent to the GMPM criterion with an infinite $\left(180^{\circ}\right)$ phase margin.

Practically, relaxing the restrictions for the minor loop gain leads to reductions in the need of filtering, so smaller and cheaper passive components can be used for this purpose.

\section{ESAC criterion}

The Energy Systems Analysis Consortium (ESAC) goes a step further in the reduction of the forbidden region. It defines a piecewise linear limit defined by three points in the s-plane:

$$
\begin{aligned}
& p 1=\left(-\frac{1}{G M}, 0\right) \\
& p 2=\left(-\cos \left(P M \frac{\pi}{180}\right), \sin \left(P M \frac{\pi}{180}\right)\right) \\
& p 3=\left(-\cos \left(P M \frac{\pi}{180}\right),-\sin \left(P M \frac{\pi}{180}\right)\right)
\end{aligned}
$$

This criterion reduces the artificial conservativeness of the GMPM criterion. However, the definition of design rules for preventing instabilities in the interconnected system is more complex. A similar stability criterion, the Root Exponential Stability Criterion (RESC), has also been proposed to improve the numerical robustness of the ESAC criterion [109].

\section{Opposing argument criterion}

The opposing argument criterion was proposed as a mean to define individual stability condition to a number of loads connected in parallel to a bus [110]. This approach is specially relevant for systems where the loads are unknown, because as long as they comply with their individual stability criterion, the interconnected system will be stable. In this case, the equivalent minor loop gain is defined by the output impedance of the source multiplied by the parallel combination of the input admittance of the loads:

$$
Z_{1} Y_{L}=Z_{1} Y_{L 1}+Z_{1} Y_{L 2}+\ldots+Z_{1} Y_{L n}
$$

where $Y_{L}$ is the parallel combination of the input admittances of $n$ loads, $Y_{L 1}, Y_{L 2}, \ldots, Y_{L n}$. The stability condition for each of the load is defined according to their rated power:

$$
\operatorname{Re}\left(Z_{1} Y_{k}\right) \geq-\frac{1}{G M} \frac{P_{L k}}{P_{S}}, \quad(k=1,2, \ldots, n)
$$

where $P_{L k}$ is the rated power of the $k$ load and $P_{S}$ is the rated power of the source.

A similar approach was proposed for the GMPM, adapting the forbidden region to the rated power of the loads, however, it was demonstrated that the combination of the admittances could enter the forbidden region [111]. Instead, the opposing argument was defined in order to assure that the combined system complies with the desired margins of stability [110]. 


\section{Maximum peak criterion}

The maximum peak criterion is a well-known tool in control engineering. Provided that the interconnected system complies with the Nyquist criterion, this method assures its satisfactory dynamic performance [82].

The forbidden region for the minor loop, defined as in (2.26), is a circle around the critical point $(-1,0)$. Related with the GMPM, it can be represented as:

$$
\begin{aligned}
\left|S_{M A X}\right|_{G M} & =\frac{1}{1+\frac{1}{G M}} \\
\left|S_{M A X}\right|_{P M} & =\frac{1}{\sqrt{2(1-\cos (P M))}}=\frac{1}{\left.2 \sin \left(\frac{P M}{2}\right)\right)}
\end{aligned}
$$

Defining the forbidden area as $\left|S_{M A X}\right|=M$ and the minor loop as $Z_{1}(j \omega) Y_{2}(j \omega)=\alpha+j \beta$, the stability condition can be expressed as:

$$
(1+\alpha)^{2}+\beta^{2}>\frac{1}{M^{2}}
$$

Therefore, the choice of $M$ determines the amount of peaking allowed in the interconnected system.

All the previous stability criteria are based on the division of the system in a source and a load, and the interface selected to create this division affects the conclusions given by these methods. Obviously, the state of stability is independent from the way the system is divided, however the margins of stability are dependent from it. This statement is easy to understand considering the maximum peak criteria. It is reasonable to think that the peaking of a system is going to be higher in an interface selected before an output filter than the peaking obtained if the interface is selected after this filter. Therefore, in general the interface selected in dc distributed systems is the bus, where all sources and loads are interconnected. In case of a multibus system, the most critical bus could be selected or the analysis can be repeated considering each bus as the interface to perform the stability analysis.

Notice that the impedance-based stability criteria reviewed can be readily implemented with blackbox models. In fact in case the G-parameters model is obtained, the output impedance and input admittance are some of the parameters identified, see (2.8).

\subsubsection{Large-signal stability analysis}

The previous methods are very powerful, however their validity is limited by the smallsignal assumption. In case the nonlinearities of the system cannot be neglected, large-signal approaches must be considered.

In EPC-based dc distribution systems, the most extended large-signal stability criteria are the Lyapunov-based methods. The Lyapunov stability criteria, also known as the Lyapunov's second method or direct method, considers a Lyapunov candidate $V(x)$, which must be a definite positive function. It states that if its derivative taken along the trajectories of the interconnected system, is negative, the system is asymptotically stable. This criterion can be represented as: 


$$
\begin{aligned}
V(x) & =0, \text { if and only if } x=0 \\
V(x) & >0, \text { if and only if } x \neq 0 \\
\text { and } \dot{V}(x) & <0, \text { for } x \neq 0
\end{aligned}
$$

where $\mathrm{x}$ is the state vector of the system. This criterion is easy to understand considering the Lyapunov candidate as a function representing the energy of the system, therefore this methods means that if the energy of the system always decreases, it will eventually end in its equilibrium point. The region of the state variables, $x$, around the equilibrium point, where (2.30) is satisfied, determines the region of asymptotic stability around the considered equilibrium point.

The Lyapunov criterion is a necessary condition, therefore nothing can be said about the region of the state variables that do not comply with (2.30). Furthermore, the region of asymptotic stability obtained depends on the choice of the Lyapunov candidate, $V(x)$. These are the reasons why the construction of the Lyapunov candidate and the conservativeness of its result are the major concerns regarding this stability method.

The most common methods to design the Lyapunov candidate are the Brayton-Moser method, the Lyapunov functions which are easily parametrized in order to apply optimization techniques to estimate the biggest region of stability, and the multimodel approach. A review about these methods applied to dc systems can be found in [112].

\section{Brayton-Moser method}

The Brayton-Moser method uses a mixed potential function as the Lyapunov candidate [113]. Applied to de distribution systems, a suitable mixed potential function would be:

$$
F(v, i)=-A(i)+B(v)+i^{T} \Lambda v
$$

where $i$ is the vector of inductive currents, $v$ is the vector of capacitive voltages, $A(i)$ and $B(v)$ can be nonlinear, and $\Lambda$ is a constant matrix. From this expression a Lyapunov candidate can be designed as:

$$
V(v, i)=\frac{\mu_{1}-\mu_{2}}{2} F(v, i)+\frac{1}{2} P_{i}^{T}[L]^{-1} P_{i}+\frac{1}{2} P_{v}^{T}[C]^{-1} P_{v}
$$

where $[L]$ and $[C]$ are the matrices related to the inductances and capacitances respectively, $i$ and $v$ are derivatives with respect to $i$ and $v$, and $\mu_{1}$ and $\mu_{2}$ are scalars defined as:

$$
\begin{aligned}
& \left.\mu_{1}=\min \left\{\lambda\left([L]^{-\frac{1}{2}} \cdot A_{i i} \cdot[L]^{-\frac{1}{2}}\right)\right\}=\min \left\{\lambda\left(M_{L}\right)\right\}\right) \\
& \left.\mu_{2}=\min \left\{\lambda\left([C]^{-\frac{1}{2}} \cdot B_{v v} \cdot[C]^{-\frac{1}{2}}\right)\right\}=\min \left\{\lambda\left(M_{C}\right)\right\}\right)
\end{aligned}
$$

where $\lambda$ represents the eigenvalues. With this Lyapunov candidate, the stability condition is as follows, if:

$$
\lambda\left(M_{L}\right)+\lambda\left(M_{C}\right)>0
$$

for all $\mathrm{i}$ and $\mathrm{v}$ within the domain $\mathrm{D}$ and: 


$$
V(i, v) \longrightarrow \infty \text { when }|i|+|v| \longrightarrow \infty
$$

then the domain D is asymptotically stable.

\section{Lyapunov functions amenable to parametrization and optimization}

The idea behind this approach is to design Lyapunov candidates such that genetic algorithms can be implemented to maximize the region of asymptotic stability. In [114] a collection of structures for Lyapunov candidates is compared in terms of complexity (number of parameters to be determined) and conservativeness (comparison between the real and estimated region of stability). Therein the use of the Jacobian matrix of the system in Jordan form is recommended, as the ellipsoidal trajectories of the system are represented as circular trajectories, simplifying the analysis. The different structures for Lyapunov candidates are described in the following.

A Full Quadratic Lyapunov Function (FQLF) has the form:

$$
V=x^{T} P x
$$

where $\mathrm{P}$ is symmetric and definite positive. Therefore the number of parameters to be estimated is:

$$
N_{T}=\frac{N(N+1)}{2}
$$

where $N_{T}$ is the total number of parameter to be estimated and $N$ is the order of the system.

A Block-Diagonalized Quadratic Lyapunov Function (BDQLF) has the form:

$$
V=\sum_{i=1}^{N_{c}} a_{c, i}\left(z_{2 i-1}^{2}+z_{2 i}^{2}\right)+\sum_{i=1}^{N_{r}} a_{r, i} z_{2 N r+i}^{2}
$$

where $N_{c}$ is the number of pairs of complex eigenvalues, $N_{r}$ is the number of real eigenvalues, $z_{i}$ are the state variables of the system in Jordan form. Therefore the number of parameters to be estimated is:

$$
N_{T}=N_{c}+N_{r}
$$

A Hyper Cylindrical Lyapunov Function (HCLF) is a non-quadratic function, such that defining the following vector:

$$
v_{i}=\left\{\begin{array}{lc}
a_{c, i}\left(z_{i-1}^{2}+Z_{2 i}^{2}\right) & i \leq N_{c} \\
a_{r, i} z_{N_{c}+i}^{2} & N_{c}<i \leq N_{c}+N_{r}
\end{array}\right.
$$

the Lyapunov function is:

$$
V=\|\mathbf{v}\|_{\infty}=\max _{i}\left|v_{i}\right|
$$

The number of parameters is the same as in the previous case (2.39). 


\section{Multimodel approach}

There is a Lyapunov method for polytopic models (2.18) [94]. A sufficient condition for asymptotic stability is the existence of a quadratic function:

$$
V(x)=x^{T} P x, P=P^{T}>0
$$

such that

$$
\dot{V}(x)<0
$$

in a certain region. For linear systems this condition can be expressed as:

$$
\dot{V}(x)=x^{T}\left(A^{T} P+P A\right) x
$$

where $A$ is the state matrix, and the condition for polytopic models becomes the LMI problem:

$$
A_{i}^{T} P+P A_{i}<0, i=1, \ldots, n
$$

The aforementioned is true for polytopic models with coincident equilibrium points. The reason is that if all the linear models share the same equilibrium point, the following holds:

$$
\begin{aligned}
\dot{\tilde{x}} & =\sum_{i=1}^{n} \omega_{i}\left(\tilde{x}+x_{e}, u+u_{e}\right)\left[A_{i} \tilde{x}+B_{i} \tilde{u}\right] \\
& +\sum_{i=1}^{n} \omega_{i}\left(\tilde{x}+x_{e}, u+u_{e}\right)\left[A_{i} x_{e}+B_{i} u_{e}+\varphi\right]
\end{aligned}
$$

where by definition

$$
A_{i} x_{e}+B_{i} u_{e}+\varphi=0
$$

for all $i$. Therefore, if the Lyapunov candidate (2.42) is considered, its derivative would have the following form:

$$
\dot{V}=\sum_{i=1}^{n} \omega_{i}\left(\tilde{x}+x_{e}, u+u_{e}\right) \tilde{x}^{T}\left[A_{i}^{T} P+P A_{i}\right] \tilde{x}
$$

Having in mind that the weighting functions are positive by definition, the condition (2.43) is only possible if the condition (2.45) is met.

To the best of the author's knowledge, in the literature there are no publications about large-signal stability analysis of EPCs-based systems using blackbox models. In this brief review of Lyapunov-based methods, it seems that the Brayton-Moser method would be difficult to apply using identification techniques because a detailed knowledge about the internal variables is needed. On the other hand, the multimodel approach seems very suitable for blackbox models as it is based on linearized models, which can be obtained applying small-signal perturbations to the EPCs. The models obtained using a blackbox 
approach are input-output models, however an estimation of the state-space model (with unknown state variables) can be deduced from this information. The regions of stability obtained for the state variables must be related to the output variables in order to extract meaningful information. Finally, the use of different Lyapunov candidates like BDQLF or HCLF in order to reduce the conservativeness in the estimation of the region of stability is considered to be an interesting research line.

\subsection{Conclusions}

In this chapter the state of the art related with this thesis has been reviewed. The information about the different architectures and control techniques proposed for this kind of system is useful to identify the phenomena that the blackbox models should be able to reproduce. Nonlinear responses are expected from the EPCs working in dc microgrids due to the substantial variability of the system. In this thesis some modeling techniques are proposed in order to take into account changes in the control mode of the converters, which could be caused, for instance, by the DBS control strategy. Besides, the validation examples used are based on the scenarios studied in this section.

The state of the art about modeling techniques for EPCs is fundamental in this thesis. Even if this work is focused on blackbox approaches, it is considered that a review of analytical techniques is important. Blackbox approaches intend to replicate the information given by analytical models without information about the internal architecture of the converters. Therefore, knowing the different analytical models that have been proposed and their purpose is useful to improve or propose novel blackbox techniques. In fact, most of the blackbox approaches have an analytical counterpart, as two-port models or polytopic models. The blackbox approaches have been classified in three groups: linear, static nonlinear, and dynamic nonlinear. Due to the strong variability expected in dc microgrids, in this thesis dynamic nonlinear models are studied.

Finally, small and large-signal stability analysis methods have been reviewed. Smallsignal approaches are well-established in the literature and the industry. Their compromise between simplicity and accuracy makes them a very powerful tool for this kind of application. Besides, blackbox approaches can be readily used for this kind of analysis. However, the nonlinearities existing in dc microgrids due to the presence of CPLs, operating point dependent EPCs, or changes of operation mode of the controllers, limit the range of application of linear approaches. Consequently, in this thesis it will be studied the use of blackbox models for large-signal stability analysis.

The review presented in this chapter about the topics related with this research is one of the contributions of this thesis. 



\section{Part II}

\section{Large-signal blackbox modeling of electronic power converters in Microgrids}





\title{
Comparison of blackbox models of electronic power converters
}

\begin{abstract}
$\mathrm{T}$
his chapter compares the performance of the existing blackbox models for EPCs in dc microgrids. The three main approaches described in Section 2.3, namely linear, static nonlinear, and dynamic nonlinear models will be obtained analytically or applying identification techniques. Different converters in various conditions will be used as testbeds, based on the review about dc microgrids characteristics. The performance of the models will be compared with the switching model of the converters. This methodology is justified because the switching model of EPCs is considered a good approximation of the actual converters, therefore the goal is to obtain blackbox models which are able to reproduce the response of this kind of model. This approach facilitates the theoretical analysis of the blackbox models for a wide range of different cases, avoiding practical difficulties. The methodology to identify small-signal models of converters around different operating points has been studied in previous works and the description can be found in the literature, as reviewed in Section 2.3.1. Consequently, the focus of this thesis is in finding suitable modeling structures able to reproduce the large-signal behavior of EPCs from this information.
\end{abstract}

\subsection{Dependence of the converters on the operating point}

In the previous chapter the nonlinear nature of the EPCs (Section 2.2) was described. This section explicitly shows the dependency of the dynamic behavior of the converters on their operating point. In order to obtain a model using identification techniques, it is very convenient to perform small-signal perturbations around an operating point in order to obtain a linear model that describes the behavior of the converter. However, the accuracy of the model will depend on the proximity of the operating conditions to the point where the model was identified. In order to illustrate the dependency of the EPCs on the operating conditions, the analytical model of the basic topologies (buck, boost, and buck-boost) will be detailed in Appendix B and represented graphically in this section. Afterwards, the blackbox models will be derived or identified and their response will be compared with the switching model in different conditions. Notice that the models identified using smallsignal perturbations around an operating point are equivalent to a linearization around an operating point of the analytical average model of the converters. 


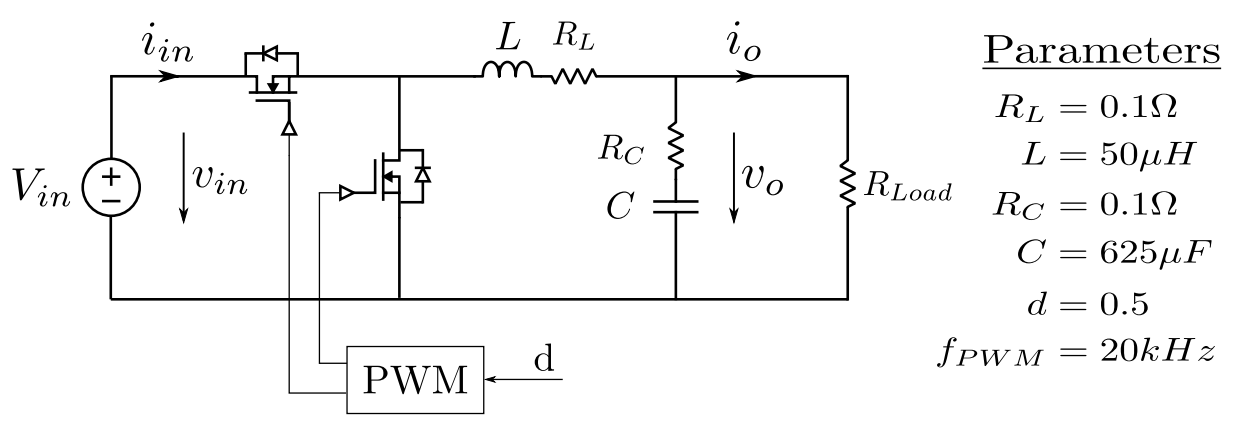

Figure 3.1: Synchronous buck converter circuit.

\subsubsection{Buck converter case}

This part studies the dynamic behavior of the buck converter in different conditions. First, a synchronous buck converter in open loop is considered. Then, the loop is closed with a PI regulator to control the output voltage. Finally, the low-side switch is substituted by a diode in order to consider continuous and discontinuous conduction modes.

\section{Synchronous buck converter in open loop}

The scheme of the synchronous buck converter implemented is depicted in Fig. 3.1. In this case the G-parameters model of this converter was obtained analytically in order to get insight into the influence of the operating conditions on the dynamic behavior of the converter. In Appendix B.1 the SSA representation of this converter is shown, as well as the derivation of the G-parameters from these equations. It can be seen that the state matrix is not affected by the duty cycle, hence the poles of the system are not affected either, as it can be seen in (B.11). The analytical expression of the G-parameters model shows that the duty cycle only modifies the gain of the transfer functions, except the output impedance which is not affected at all. This effect is shown graphically in Fig B.2, where the bode plots of the G-parameters models are presented for different values of the duty cycle.

This information suggests that a small-signal model should capture the dynamic behavior accurately as long as the duty cycle is kept constant, as the output variables do not appear in the equations.

The G-parameters model has two inputs, the input voltage and the output current. The input voltage is measured from the voltage source, however the output current depends directly on the output voltage and the load impedance. Therefore, the output voltage of the G-parameters model is imposed to the load with a controlled voltage source and the resulting output current is measured and fed back to the input of the model. Consequently, the input of the model instead of being the output current is the impedance of the load. This approach is very useful to interconnect different two-port models. Finally, the input current is the other output variable, which could be imposed in the input port with a controlled current source in case of interconnected systems.

In Fig. 3.2 the response of the switching model of the converter is compared with the G-parameters model, which was obtained with the equations shown in (B.11) evaluated with the parameters shown in Fig 3.1. It can be seen that the model captures the average dynamic behavior of the converter perfectly under a step in the input voltage at time $t=1 \mathrm{~ms}$, and a load step at time $t=6 \mathrm{~ms}$. 

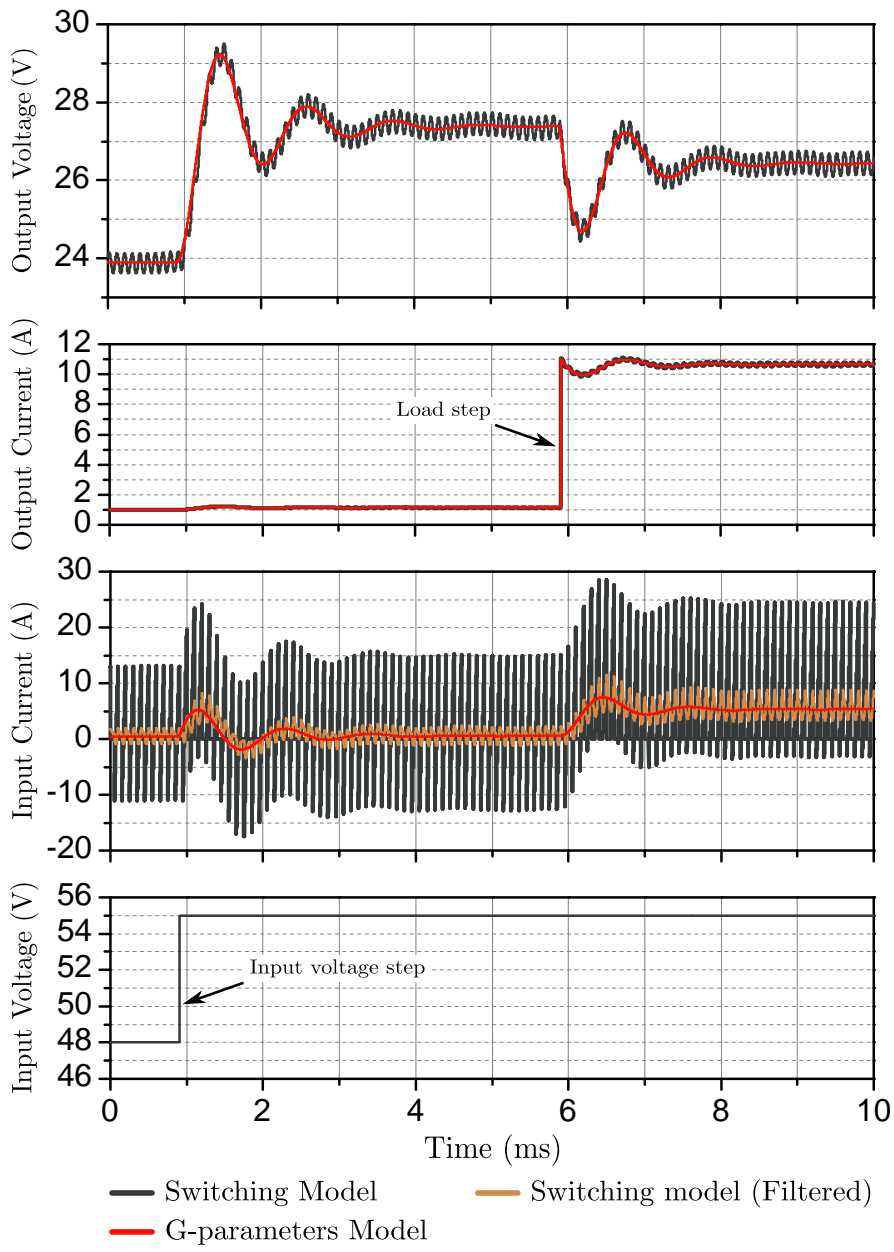

Figure 3.2: Comparison between switching model and G-parameters model of a synchronous buck converter in open loop.

\section{Voltage-controlled synchronous buck converter}

The next step is to close the loop and analyze the effect of the control loop in the dynamic behavior of the converter (Fig. 3.3). When the loop is closed, the duty cycle becomes another variable of the system, hence the product of the duty cycle by the variables of the system must be linearized around a particular value. In Appendix C.1 the SSA representation of a voltage-controlled synchronous buck converter is derived. In (C.6) it can be seen that the input voltage affects the state matrix, so the position of the poles of the system will depend on the operating point of this variable. The input and output matrices depend on the value of both input variables, so in general the dynamic of the converter will depend on the operating point.

In Fig. 3.4 the switching model is compared with the G-parameters model, the WienerHammerstein model, and the polytopic model. The G-parameters model was obtained analytically with the equations shown in (C.6). The Wiener-Hammerstein model was identified from the response of the converter to steps in the input variables. The structure used is the one shown in (2.10) and the parameters identified are presented in Table 3.1. The polytopic model consists of four G-parameters models obtained around the initial and final operating point, i.e. $V_{\text {in }}=\{40,48\}$ and $I_{\text {out }}=\{1,10\}$, the transfer functions derived are presented in Table. C.1 and Table. C.2 and their bode plots are represented in Fig. C.2. 


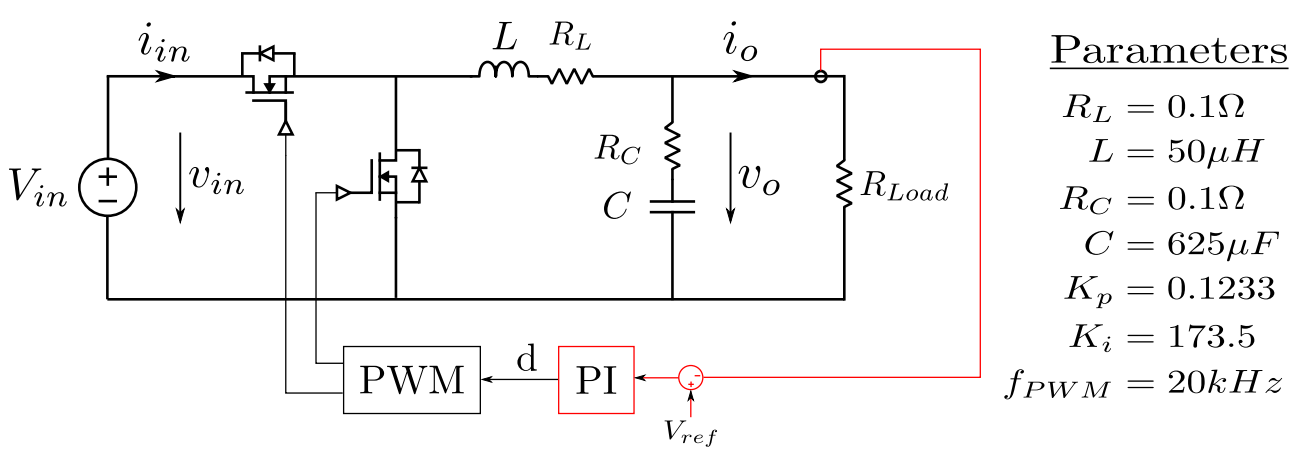

Figure 3.3: Voltage-controlled synchronous buck converter circuit.

Table 3.1: Parameters of the Wiener-Hammerstein model of the voltage-controlled synchronous buck converter

\begin{tabular}{|c|c||c|c||c|c|}
\hline \multicolumn{2}{|c||}{ Output filter } & \multicolumn{2}{c||}{ Input filter } & \multicolumn{2}{c|}{ Audiosuscentibility } \\
\hline \hline$L_{\text {out }}$ & $9.24 \mu \mathrm{H}$ & $L_{\text {in }}$ & $327 \mu \mathrm{H}$ & $L_{G}$ & $60 \mu \mathrm{H}$ \\
\hline$R_{L}$ & $2.8 \Omega$ & $R_{\text {in }}$ & $0.76 \Omega$ & $R_{G}$ & $0.05 \Omega$ \\
\hline$C_{\text {out }}$ & $580.2 \mu \mathrm{F}$ & $C_{\text {in }}$ & $15.6 \mu \mathrm{F}$ & $C_{G}$ & $0.2 \mathrm{~F}$ \\
\hline$R_{C}$ & $0.02 \Omega$ & & & & \\
\hline
\end{tabular}

Regarding the weighting functions, double sigmoids were used with a center in the middle between the two values considered for each variable, i.e. $V_{\text {center }}=44 \mathrm{~V}$ and $I_{\text {center }}=5.5 \mathrm{~A}$, and a slope of 2 (see (2.19)).

In Fig. 3.4 the comparison between the switching model and the three blackbox modeling approaches is presented. Besides, the error of the models in the estimation of the output variables is also shown, where the mean error of the models during each step is highlighted. The figure shows how the models with a linear dynamic (G-parameters and Wiener-Hammerstein) have errors in the amplitude and frequency of the oscillations during the step in the input voltage $(t=1 \mathrm{~ms})$, as it was expected from the equations. It can be seen that the mean error during the input voltage step of the polytopic model is a $41.3 \%$ lower than the G-parameters model and 34.1\% lower than the Wiener-Hammerstein model for the output voltage. Regarding the input current, the Wiener-Hammerstein and polytopic models have a similar performance with an error $24.2 \%$ lower than the G-parameters model. The G-parameters model has an additional deviation in the steady state value of the input current, whereas the Wiener-Hammerstein model captures the static value of the input current after each perturbation.

At time $t=6 \mathrm{~ms}$ a load step occurs. In this case, the G-parameters and WienerHammerstein models have a similar performance in terms of error, whereas the polytopic model is able to adapt the frequency of the oscillations, obtaining the best performance in the estimation of the dynamic behavior of the converter. In particular, an improvement around $55.7 \%$ is obtained by the polytopic model compared with the other two approaches for the output voltage.

A graphical comparison of different measures of the overall error (maximum and mean errors, and standard deviation) between the switching model and the blackbox models is given in Fig. 3.5. It can be seen that the polytopic model has clearly the lowest error in the estimation of the output voltage with an improvement between 30 and $50 \%$. Regarding the input current, the polytopic and Wiener-Hammerstein model have a similar performance, 

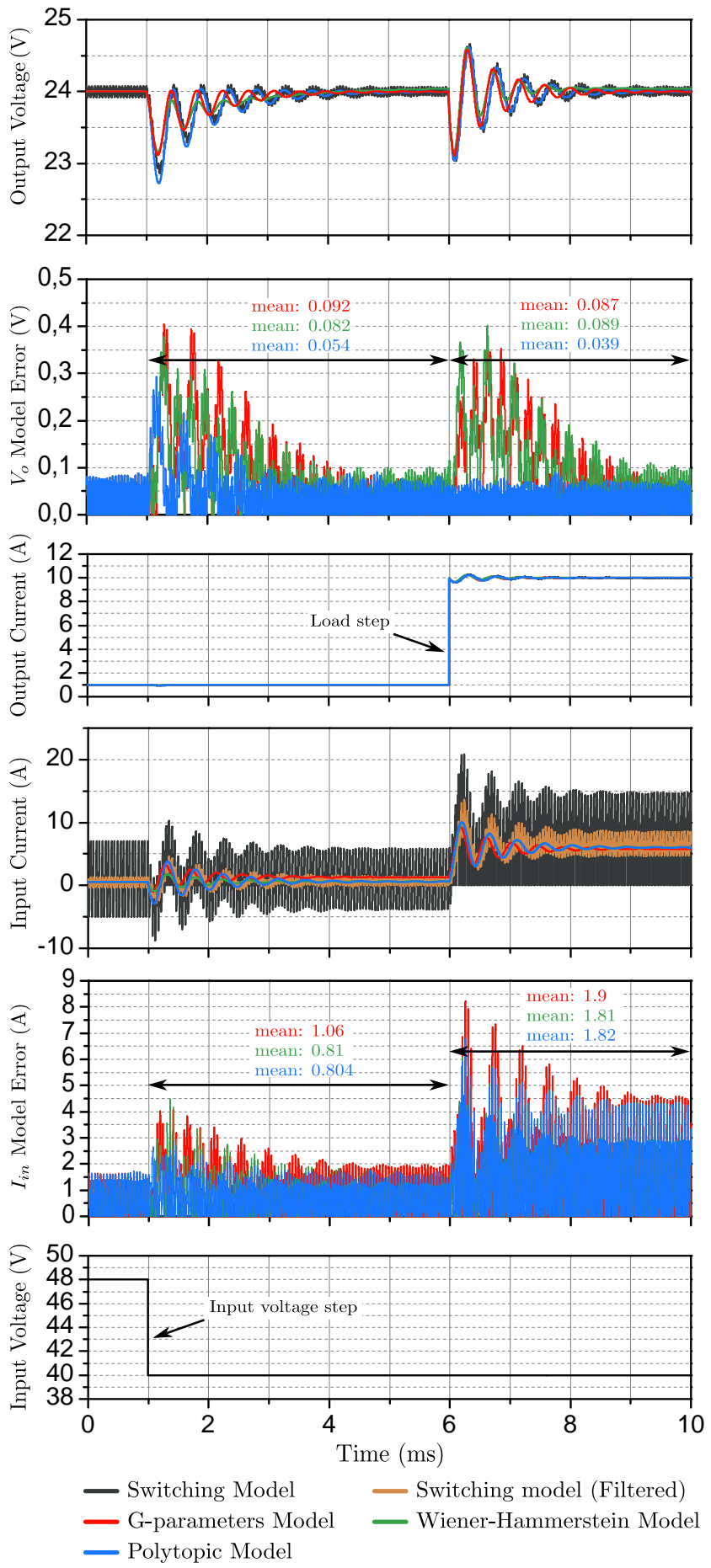

Figure 3.4: Comparison between switching model and the three types of blackbox structures of a voltagecontrolled synchronous buck converter. 

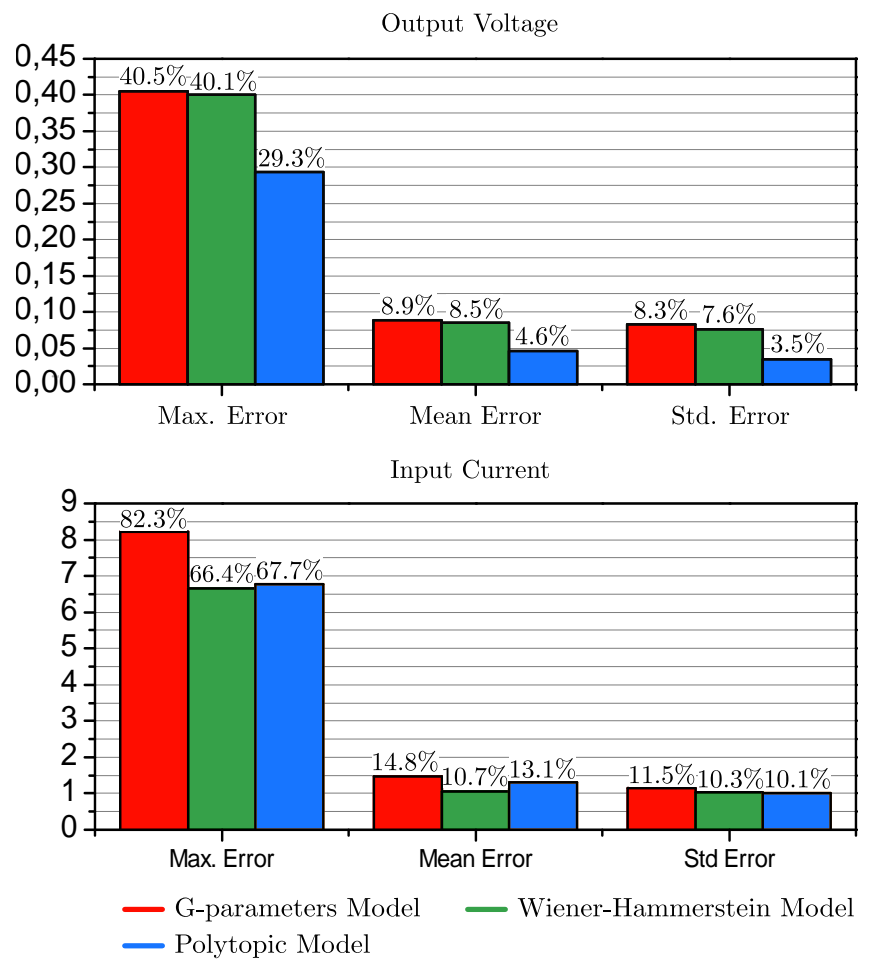

Figure 3.5: Error comparison between switching model and the three types of blackbox structures of a voltage-controlled synchronous buck converter.

which is around a $20 \%$ better than the G-parameters model. This difference is in part due to the errors in the steady state response of the G-parameters model, which are not present in the other two approaches.

Furthermore in this figure the relative error is presented, which is related with the maximum deviation of the switching model during the transient response. The maximum deviation in the output voltage is $1 \mathrm{~V}$, whereas the maximum deviation in the input current is $10 \mathrm{~A}$.

Finally, in terms of computational burden, the models are compared taking into account the time to complete the simulation shown. The simulation time of the switching model was $11 \mathrm{~s}$, whereas the G-parameters, Wiener-Hammerstein, and polytopic models took $2 \mathrm{~s}$, $1 \mathrm{~s}$, and $3 \mathrm{~s}$, respectively. In general, the computational burden of the switching model will be higher than the average blackbox models precisely due to the switching process. Consequently, the integration time of the simulation of the switching model must be between 10 and 100 times lower than the switching frequency, whereas the integration time of the blackbox models are only related with the averaged dynamic behavior of the system. In this case, the minimum integration time for the switching model was $0.1 \mu \mathrm{s}$, whereas for the blackbox models was $1 \mu s$.

\section{Voltage-controlled buck converter in continuous and discontinuous conduction modes}

In this case the low-side switch is replaced by a diode in order to study the dynamic of the converter in Discontinuous Conduction Mode (DCM) and Continuous Conduction Mode (CCM) (Fig. 3.6). It is well-known that with this configuration the inductor current cannot be negative. Therefore, in case the current ripple in the inductor reaches zero during a switching interval, the converter enters in discontinuous conduction mode. In this 


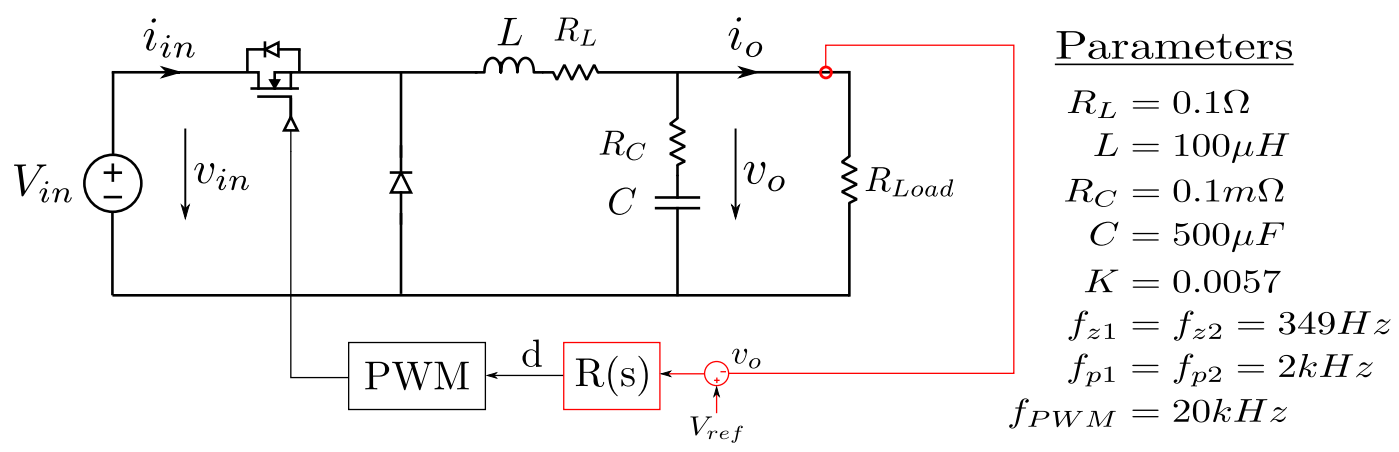

Figure 3.6: Non-synchronous buck converter circuit.

operation mode, the behavior of the converter depends on the amount of time that the inductor current is zero, which depends in turn on the load demand.

The converter regulates the output voltage using a type 3 regulator with the following transfer function:

$$
R(s)=K \frac{1+\frac{s}{2 \pi f_{z 1}}}{\frac{s}{2 \pi f_{z 1}}} \frac{1+\frac{s}{2 \pi f_{z 2}}}{\left(1+\frac{s}{2 \pi f_{p 1}}\right)\left(1+\frac{s}{2 \pi f_{p 2}}\right)}
$$

where the parameters of the regulator are shown in Fig. 3.6. The models were obtained using identification methods. For the sake of clarity in this case only the G-parameters and polytopic models will be compared, as the main concern will be in the estimation of the dynamic behavior of the converter. Besides, as the mode of operation (continuous or discontinuous) is mainly related to the load current, the focus of the comparison will be on the transfer functions related to these variables, i.e. the output impedance and the back current gain (see (2.8)).

Fig. 3.7 shows the frequency response of the converter for different values of the output current, $I_{o}=\{1,2,3,4\} A$. The amplitude of the sinusoidal signal used for the perturbation was $0.1 A$ for all the cases, which is a value big enough to generate a response higher than the switching ripple and small enough to obtain a linear behavior. The corresponding transfer functions were identified from these frequency responses using the System Identification Tool of Matlab. The response of the transfer functions identified for each of the smallsignal frequency responses is shown overlapping the measured signal. It can be seen how the identified transfer functions represent very accurately the small-signal frequency response of the converter around different operating points. Some differences can be observed in the high frequency range of the back current gain, however in this range the magnitude is below $-30 d B$, hence its effect in the dynamic response will be negligible. Table 3.2 shows the parameters of the identified transfer functions.

Finally, a polytopic model is generated using the transfer functions identified around the different values of the output current and integrating them together by means of double sigmoid weighting functions (see (2.19) and (2.20)). The values of the center of the sigmoid selected are the middle point among the values of the output current and the slope is equal to 10 in all the cases.

The converter works in DCM for values of the output current below $I_{O}=3 \mathrm{~A}$, whereas it works in CCM when the output current is above this value. Within the DCM the converter changes its dynamic behavior substantially depending on the value of the output current. 


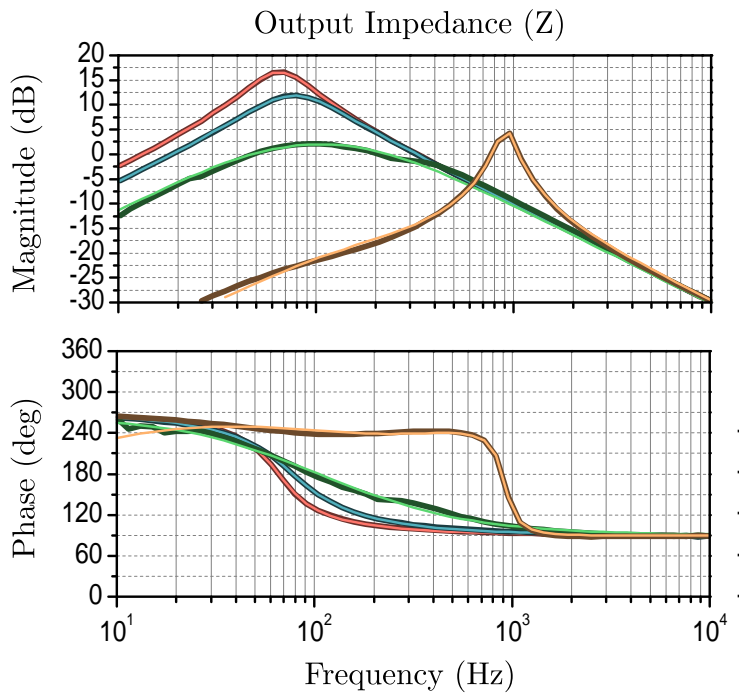

Frequency $(\mathrm{Hz})$
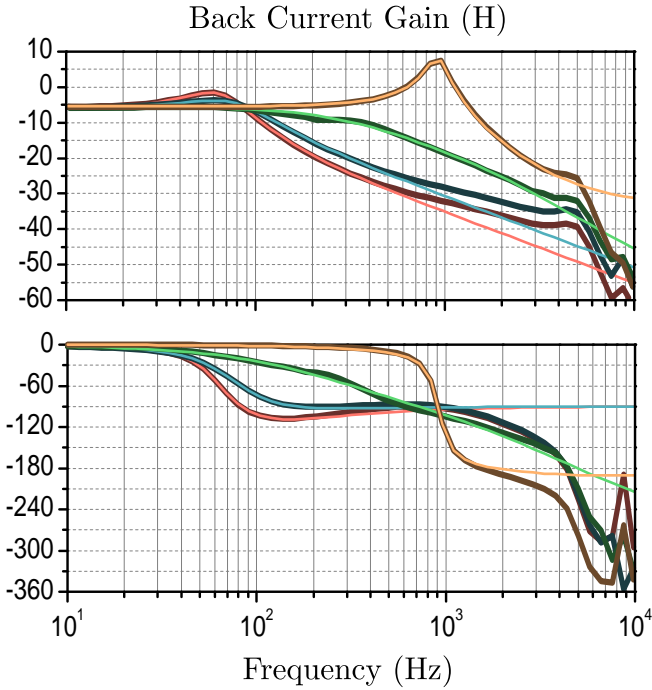

Frequency $(\mathrm{Hz})$

$$
\begin{array}{ll}
\text { - Switching model }\left(I_{o}=1 A\right) & \text { - Identified model }\left(I_{o}=1 A\right) \\
\text { - Switching model }\left(I_{o}=2 A\right) & \text { - Identified model }\left(I_{o}=2 A\right) \\
\text { - Switching model }\left(I_{o}=3 A\right) & \text { - Identified model }\left(I_{o}=3 A\right) \\
\text { - Switching model }\left(I_{o}=4 A\right) & \text { - Identified model }\left(I_{o}=4 A\right)
\end{array}
$$

Figure 3.7: Frequency response of the buck converter for different values of the output current. Comparison between the measured signal and the identified transfer functions.

Table 3.2: Parameters of the identified transfer functions of the voltage-controlled buck converter for different values of the output current.

\begin{tabular}{|c||c||c|}
\hline Output Current & Output impedance, Z(s) & Back current gain, $\mathbf{H}(\mathbf{s})$ \\
\hline \hline$I_{o}=1 A$ & $\frac{1983.8 s-544.5}{s^{2}+291.5 s+169732.1}$ & $\frac{109.4 s+85420.6}{s^{2}+293 s+168123.4}$ \\
\hline$I_{o}=2 A$ & $\frac{1982.4 s-832.4}{s^{2}+505.3 s+239063.6}$ & $\frac{182.3 s+117577.6}{s^{2}+505.6 s+230934}$ \\
\hline$I_{o}=3 A$ & $\frac{1914.4 s+5103.1}{s^{2}+1521.7 s+438243.6}$ & $\frac{-270.6 s+13941816.8}{s^{2}+18312.8 s+27096075.8}$ \\
\hline$I_{o}=4 A$ & $\frac{2086.7 s^{2}+4643 e 3 s+195 e 6}{s^{3}+2092.9 s^{2}+34 e 6 s+27.7 e 9}$ & $\frac{-20.2 e 3 s^{2}-209 e 6 s+15813 e 9}{s^{3}+889 e 3 s^{2}+1125 e 6 s+29524 e 9}$ \\
\hline
\end{tabular}


However, in the CCM the dependency of the dynamic behavior of the converter with the output current is much lower. Due to the strong differences in the dynamic behavior of the converter, this case is considered very interesting and the comparison among the smallsignal models, the polytopic model, and the switching model will be detailed thoroughly.

In Fig. 3.8 the response of the switching model of the converter is compared with each of the small-signal models and the polytopic model. The initial operating point is $I_{O}=$ $1 \mathrm{~A}$, whereas the input voltage will be kept constant and equal to $V_{\text {in }}=48 \mathrm{~V}$. In these conditions the converter works in DCM, as it can be confirmed looking at the inductor current (which reaches zero within the switching period). The load is varied by adding or removing resistors in parallel with the load. At time $t=1 \mathrm{~ms}$ a resistor is connected, increasing the output current to $I_{o}=1.2 \mathrm{~A}$. It can be seen that the small-signal model obtained around $I_{o}=1 \mathrm{~A}$ is the one that captures the dynamic behavior of the converter with better accuracy. Regarding the polytopic model, the weighting function related to the model obtained around $I_{o}=1 A$ is equal to 1 , so its accuracy is equally high.

At time $t=11 \mathrm{~ms}$ another resistor is added increasing the output current to $I_{o}=4.7 \mathrm{~A}$. This load step leads the converter to make a transition from DCM to CCM. It can be seen how none of the small-signal models are able to reproduce the dynamic response of the converter during this transition. Besides, the polytopic model has a very poor accuracy in this transition, as the weighting function of the model obtained around $I_{o}=4 \mathrm{~A}$ (where the converter works in CCM) suddenly reaches 1 following the sharp variation of the output current. However, the behavior of the switching model seems to make a transition among the small-signal models or, in other words, it seems to modify its dynamic behavior with a certain dynamic.

In order to check the accuracy of the small-signal model obtained in CCM, another resistor is added at time $t=26 \mathrm{~ms}$, increasing the output current to $I_{o}=5.3 \mathrm{~A}$. It can be observed that in this case the small-signal model obtained around $I_{o}=4 \mathrm{~A}$ is the one that approximates the average behavior of the converter with high accuracy, as does the polytopic model because the weighting function related to this small-signal model is the one equal to 1 for this operating point. Finally, at time $t=36 \mathrm{~ms}$, the last two resistors are disconnected and the output current goes down to $I_{o}=1.2 \mathrm{~A}$, leading the converter to make a transition from CCM to DCM. The comparison shows that none of the smallsignal models are able to follow the dynamic behavior of the switching model. Again, the polytopic model has a poor accuracy, adopting sharply the dynamic behavior of the smallsignal model around $I_{O}=1 \mathrm{~A}$, and then, making a transition between this small-signal model and the one obtained around $I_{o}=2 A$, according to the behavior of the output current. However, as in the previous transition between conduction modes, the converter seems to make a transition among the dynamic behavior of the small-signal models, but, in this case, with a different rate of change.

The analysis of the errors between the switching model and the different G-parameters models and the polytopic model is shown in Fig. 3.9. It can be seen that during the smallsignal steps $(t=1 \mathrm{~ms}$ and $t=26 \mathrm{~ms})$ the small-signal models around the corresponding operating points $\left(I_{o}=1 \mathrm{~A}\right.$ and $I_{o}=4 \mathrm{~A}$, respectively) have a very low error and, accordingly, so it does the polytopic model. However, during the large-signal steps $(t=11 \mathrm{~ms}$ and $t=36 \mathrm{~ms}$ ) none of the models are able to represent the transient behavior of the converter.

Finally, in Fig. 3.10 the measures of the overall error between the switching model and the blackbox models are presented. It can be seen that the maximum error of the models is even higher than the maximum deviation of the switching model during the transient response. The performance of the polytopic model is not the best in this case, which corresponds to the model obtained in $I_{o}=3 \mathrm{~A}$, for the output voltage and the model obtained in $I_{o}=2 A$ for the input current. 

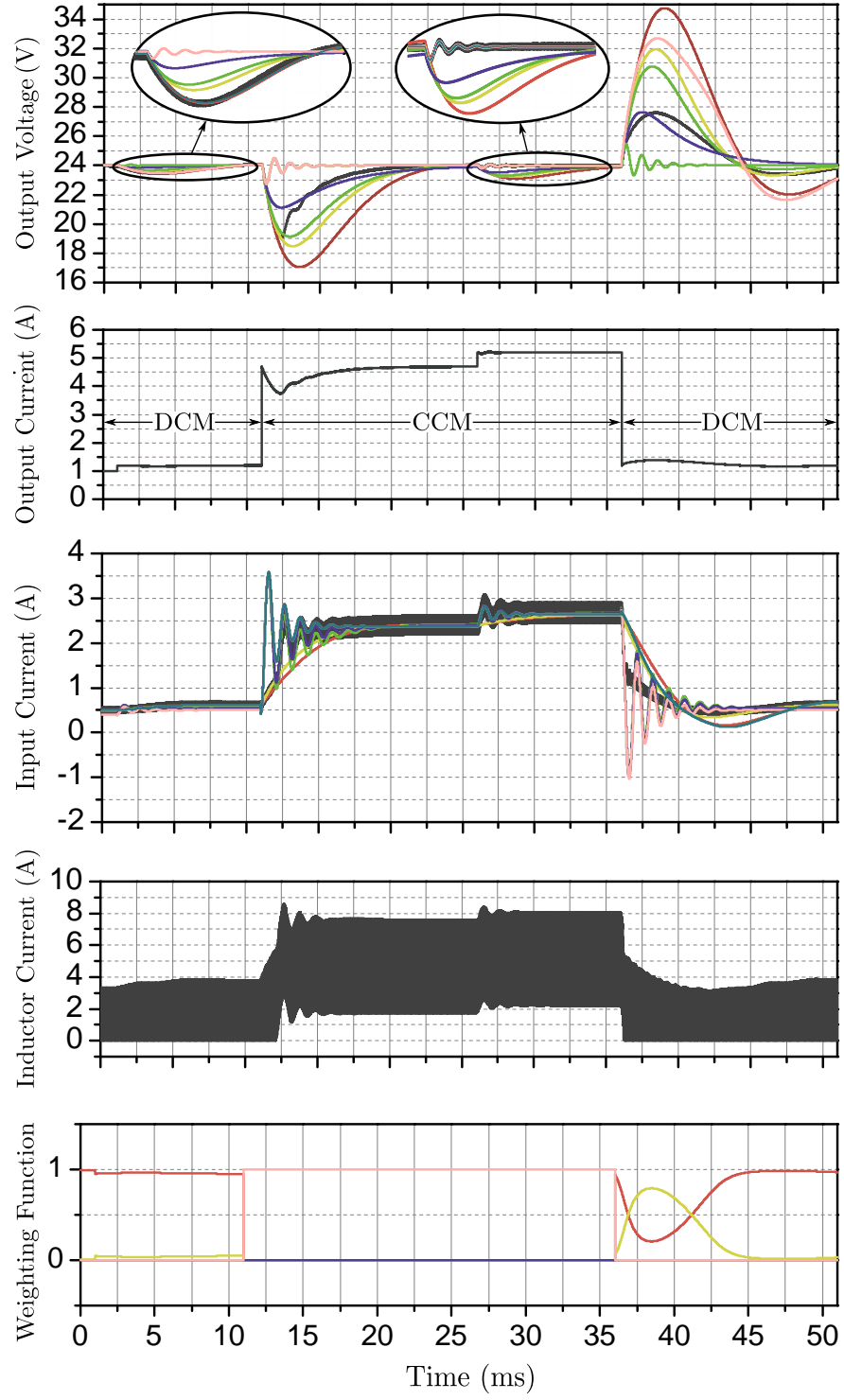
- Switching Model
- G-parameters (1A) Model
- G-parameters (2A) Model — G-parameters (2.75A) Model
- G-parameters (3A) Model — G-parameters (4A) Model
— Polytopic Model

Figure 3.8: Comparison between switching model and the three types of blackbox structures of a voltagecontrolled buck converter in DCM and CCM. 

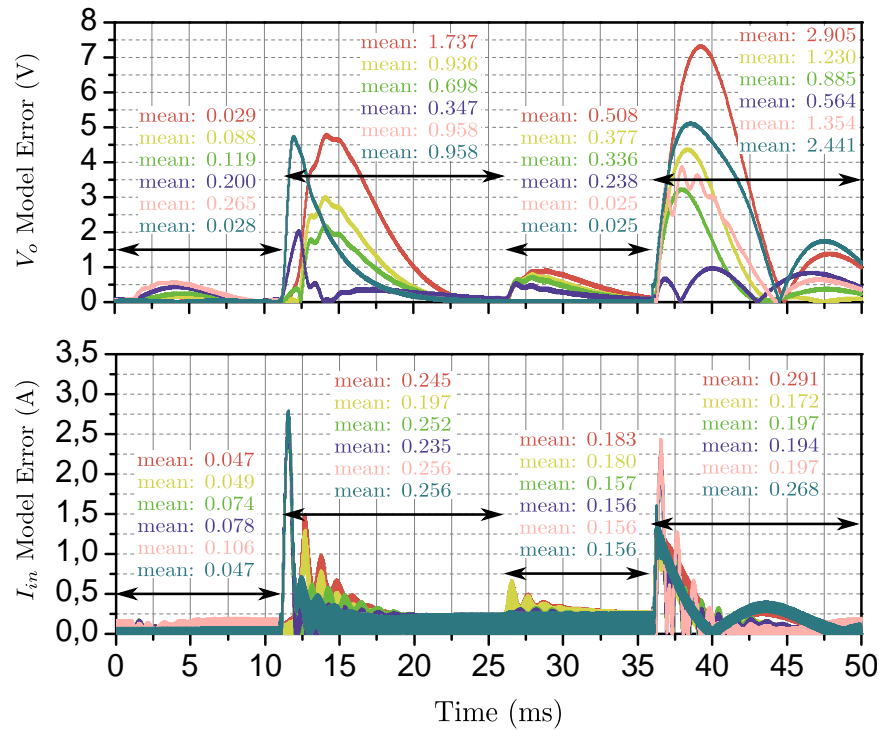

- G-parameters (1A) Model — G-parameters (2A) Model

- G-parameters (2.75A) Model — G-parameters (3A) Model

- Polytopic Model

- G-parameters (4A) Model

Figure 3.9: Error between the switching model and the three types of blackbox structures of a voltagecontrolled buck converter in DCM and CCM.
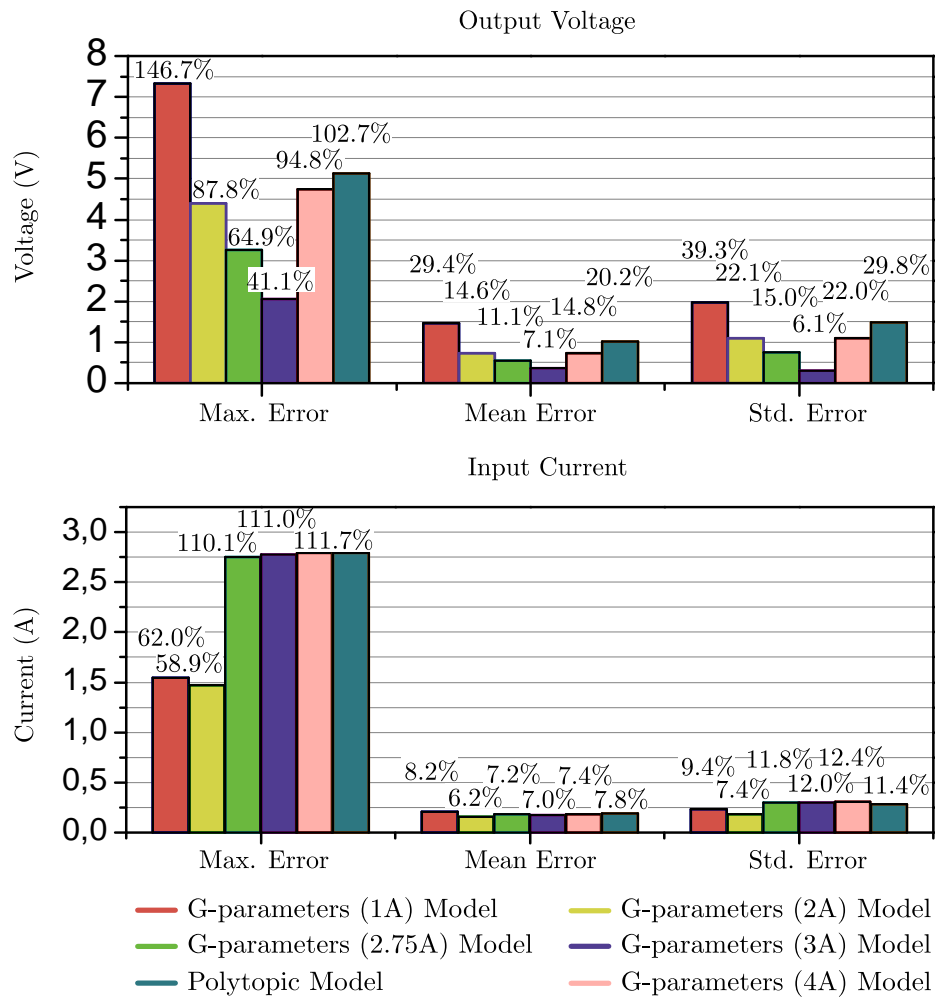

Figure 3.10: Error comparison between switching model and the three types of blackbox structures of a voltage-controlled buck converter in DCM and CCM. 
This example shows how, by making small perturbations around different operating points, it is possible to obtain small-signal models that represent accurately the behavior of the converter around these operating points. However, the accuracy of the model will depend on the variability of the dynamic behavior of the converter and the proximity of the operating conditions to the particular operating point where the converter was identified. The polytopic model is able to adapt its dynamic behavior according to the variations in the input variables of the converter, so it is able to capture the dynamic of the converter with small perturbations around different operating points. However, when a large-signal step occurs, the polytopic model fails to follow the dynamic of the converter. This effect was illustrated in the transitions from DCM to CCM and from CCM to DCM.

This case clearly shows that a new modeling approach is needed to represent converters with strong nonlinearities in their dynamic behavior, as it is the case of converters with continuous and discontinuous conduction modes.

\subsubsection{Boost converter case}

This part studies the dynamic behavior of the boost converter in open and closed loop. The boost converters dynamic is much more dependent on the operating point than in the case of buck converters, as it will be shown below. As in the previous case, the performance of the existing blackbox modeling approaches will be compared with the switching model response in order to assess their capabilities in different scenarios.

\section{Synchronous boost converter in open loop}

First the open loop characteristic of the converter is studied. Fig. 3.11 shows the scheme of the circuit implemented. As in the previous case, the converter has been analyzed analytically in order to study the influence of the operating points on its dynamic behavior. In Appendix B.2 the SSA representation of the boost converter in open loop is described and the G-parameters are derived. In this case, the duty cycle appears in the state matrix, hence the placement of the poles will depend on its value. The duty cycle also appears in the input and output matrices, but in this case multiplied by the parasitic resistance of the output capacitor, so it is expected to affect less the zeros of the transfer functions. These effects can be confirmed by checking the analytical G-parameters transfer functions ((B.17) and (B.18)), where the strong dependency of the natural frequency and the damping factor on the duty cycle is explicitly exposed. Besides the duty cycle also affects considerably the gain of the audiosusceptibility and the buck current gain. These conclusions are demonstrated graphically in Fig. B.4, where the bode plot of the G-parameters are presented for different values of the duty cycle.

The analytical G-parameters model has been evaluated with the parameters shown in Fig. 3.11 and its dynamic response to steps in the input variables has been compared with the switching model. Fig. 3.12 shows the responses of the switching model and the Gparameters model. At time $t=1 \mathrm{~ms}$ the input voltage has a negative step to $V_{o}=20 \mathrm{~V}$, whereas at time $t=6 \mathrm{~ms}$ the input voltage is restored with another step. At time $t=11 \mathrm{~ms}$ a resistor is added in parallel with the output of the converter, increasing the output current from $I_{o}=1 A$ to $I_{o}=4 A$. It can be seen how the G-parameters model reproduce perfectly the dynamic behavior of the converter.

As in the case of the buck converter in open loop, the nonlinearities in the system come from the product of the duty cycle with the variables of the converter. In the openloop simulations the duty cycle is constant, therefore the equations become linear and the dynamic behavior of the converters can be represented with transfer functions. 


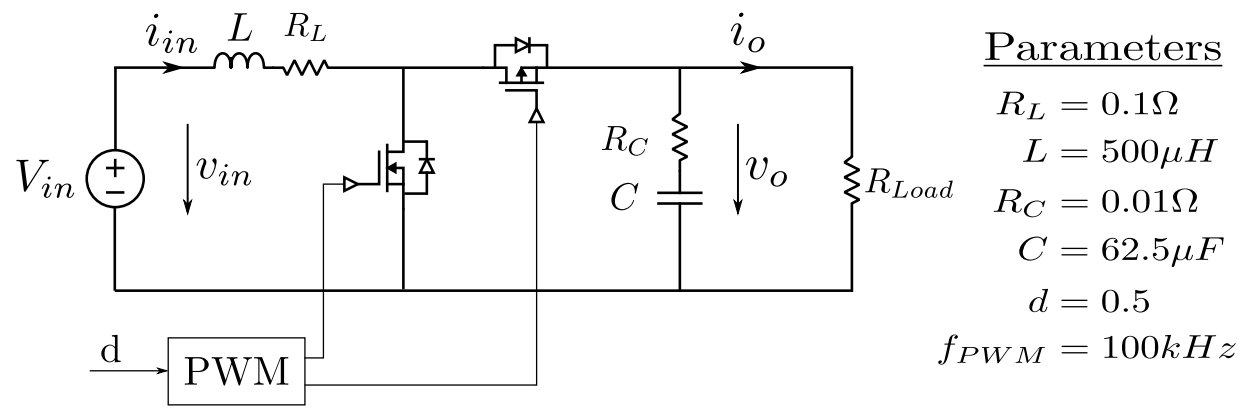

Figure 3.11: Synchronous boost converter circuit.
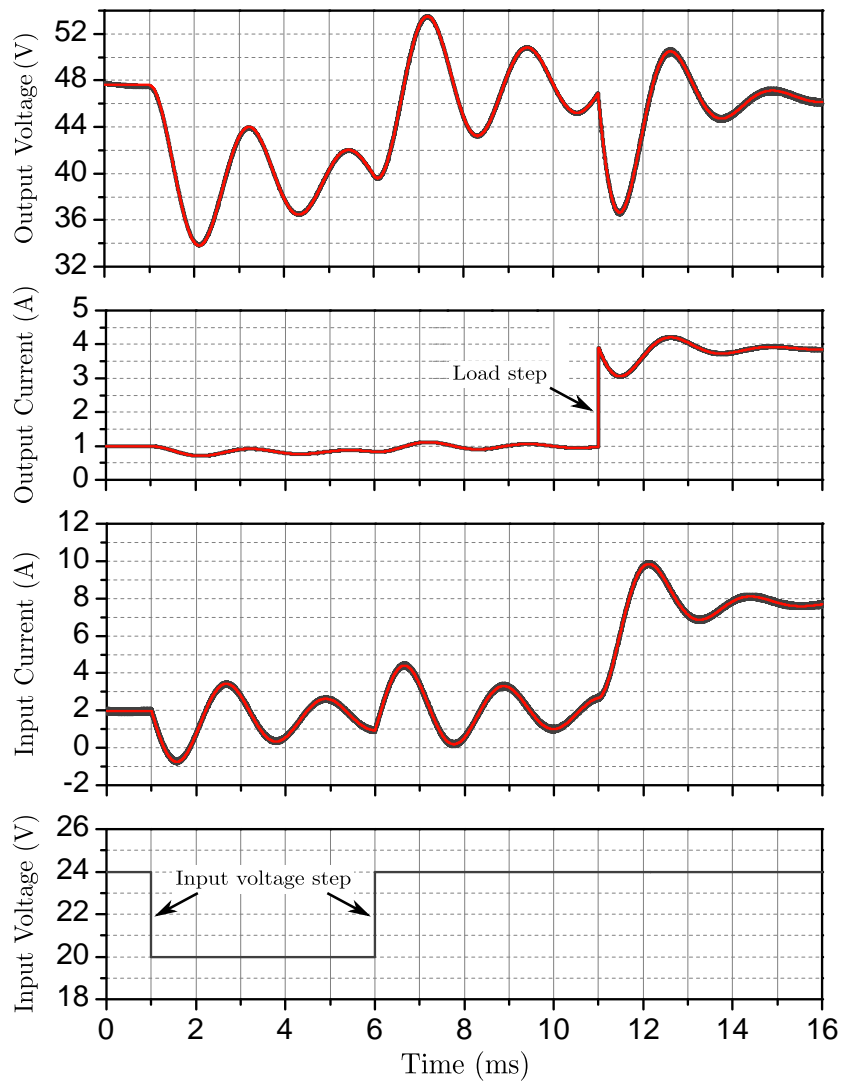

- Switching Model — G-parameters Model

Figure 3.12: Comparison between switching model and G-parameters model of a synchronous boost converter in open loop. 


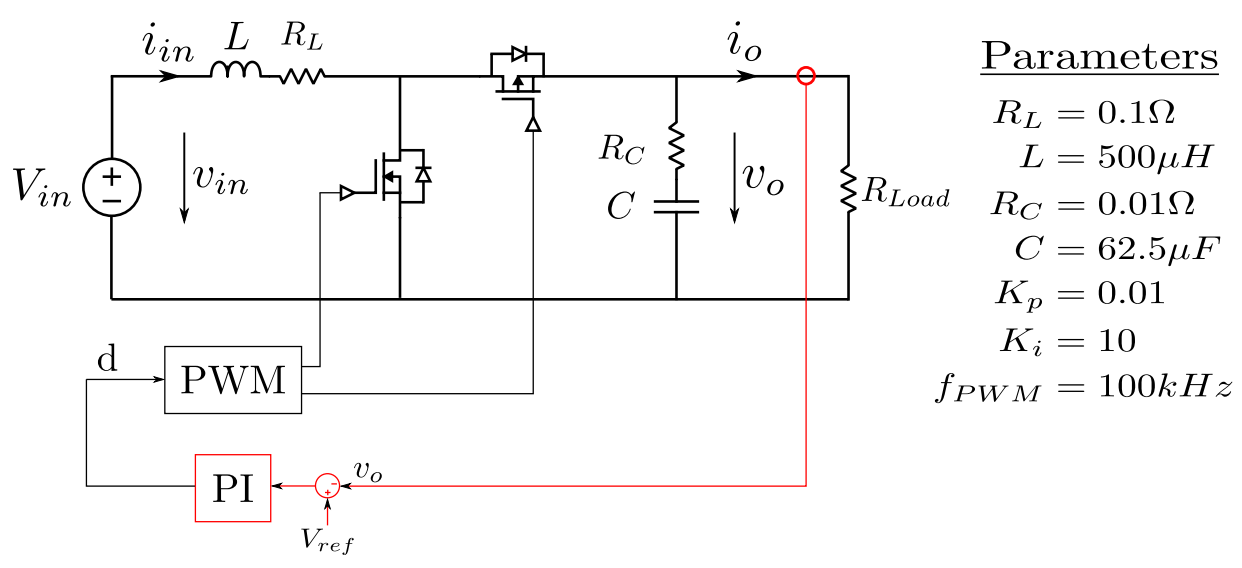

Figure 3.13: Voltage-controlled synchronous boost converter circuit.

\section{Voltage-controlled synchronous boost converter}

The next step is to close the loop, so the duty cycle varies and the system becomes nonlinear. Fig. 3.13 shows the scheme of the voltage-controlled synchronous boost converter regulated with a PI controller. In Appendix C.2 the SSA representation of the converter is derived. In (C.9) it can be seen that the state matrix is affected by the two inputs of the G-parameters model, so the operating point is expected to affect the placement of the poles of the system. Also, the input and output matrices depend on the operating point of the input of the model, but again, it is multiplied by parasitic resistances, so its effect on the zeros will be low. Again, these conclusions can be seen graphically in Fig. C.4, where the bode plots of the G-parameters are presented for different operating points.

The G-parameters models used in the comparison will be obtained using the analytical equations by changing the linearization point. Different operating points of the two inputs are considered. In particular the points selected are the nine combinations of $V_{\text {in }}=\{20,22,24\} V$ and $I_{\text {out }}=\{1,5,9\} A$. As small-signal model, the G-parameters model obtained for $V_{\text {in }}=24 \mathrm{~V}$ and $I_{\text {out }}=1 \mathrm{~A}$ is used. The nine small-signal models are integrated in a polytopic structure, using the middle points between operating points as center of the weighting functions and a slope of 4 for both variables (see (2.19) and (2.20)). The parameters of the transfer functions are shown in Table C.3 and Table C.4, which have been obtained evaluating the analytical equations with the parameters given in Fig. 3.13 and around the operating points mentioned. However, the Wiener-Hammerstein model was obtained using identification techniques from the response of the converter to steps in the input voltage and the output current. The output filter of the Wiener-Hammerstein model shown in Fig. 2.10 is of second order and it was not able to reproduce the response of the converter correctly, therefore an extra inductor $\left(L_{\text {out } 2}\right)$ was included in series with the resistor in parallel with the output inductance $\left(R_{L}\right)$. The parameters obtained in the identification are shown in Table 3.3.

Figure 3.14 shows the comparison between the switching model and the three models obtained. The initial operating point is the one selected for the small-signal G-parameters model, $V_{i n}=24 V$ and $I_{o}=1 \mathrm{~A}$. At time $t=1 \mathrm{~ms}$ the input voltage has a negative step to $V_{i n}=20 \mathrm{~V}$, where it can be seen that the G-parameters and the Wiener-Hammerstein models start losing accuracy, whereas the polytopic model is able to follow the dynamic behavior of the transition. In particular, the mean error of the polytopic model in this transition has a $68.4 \%$ less error than the G-parameters model and a $62.4 \%$ less error than the Wiener-Hammerstein model for the output voltage. Regarding the input current the improvements are of $46.9 \%$ and $49.4 \%$, respectively. 
Table 3.3: Parameters of the Wiener-Hammerstein model of the voltage-controlled synchronous boost converter

\begin{tabular}{|c|c||c|c||c|c|}
\hline \multicolumn{2}{|c||}{ Output filter } & \multicolumn{2}{c||}{ Input filter } & \multicolumn{2}{c|}{ Audiosuscentibility } \\
\hline \hline$L_{\text {out }}$ & $984.6 \mu \mathrm{H}$ & $L_{\text {in }}$ & $57.2 \mu \mathrm{H}$ & $L_{G}$ & $402.8 \mu \mathrm{H}$ \\
\hline$R_{L}$ & $1.92 \Omega$ & $R_{\text {in }}$ & $0.076 \Omega$ & $R_{G}$ & $0.9 \Omega$ \\
\hline$C_{\text {out }}$ & $302.6 \mu \mathrm{F}$ & $C_{\text {in }}$ & $1.2 \mathrm{mF}$ & $C_{G}$ & $0.22 \mathrm{~F}$ \\
\hline$R_{C}$ & $8.7 \mu \Omega$ & & & & \\
\hline$L_{\text {out } 2}$ & $1.2 \mathrm{mH}$ & & & & \\
\hline
\end{tabular}

At time $t=6 \mathrm{~ms}$ a resistor is connected in parallel with the output, making the output current increase to $I_{o}=9 \mathrm{~A}$. In this case the error of the G-parameters and WienerHammerstein models become higher in the transient response. Besides, the G-parameters model starts to fail also in the steady-state of the input current, whereas the WienerHammerstein model is able to track this value due to the nonlinear reference included in the input network (see Fig. 2.10). The polytopic model is more accurate, however some errors can be noticed at the beginning of the transition: in the output voltage the overshoot of the model is higher than the switching model; and in the input current there is an initial negative step. The reason for the initial negative step in the input current is that, due to the sharp step in the output current, the weighting functions change both sharply, changing abruptly the output of the model from the G-parameters model obtained in $V_{i n}=20 \mathrm{~V}$ and $I_{o}=1 \mathrm{~A}$ (the operating point before the load step) to the one obtained for $V_{i n}=20 \mathrm{~V}$ and $I_{o}=9 \mathrm{~A}$. As this second model has static errors in the input current, the initial point of this variable is not at the same value. The relative error analysis shows a reduction of error of the polytopic model of $58.7 \%$ and $55.5 \%$ compared with the G-parameters and WienerHammerstein models for the output voltage. Regarding the input current the improvement is $76.7 \%$ and $62.5 \%$ respectively.

Finally, at time $t=11 \mathrm{~ms}$ there is another step in the input voltage towards the initial value $V_{i n}=24 \mathrm{~V}$ and the results are similar, the G-parameters and the Wiener-Hammerstein models do not capture accurately the dynamic behavior, whereas the polytopic model does it better, but with errors in the initial part of the transitory response. In this case the error in the steady-state value of the input current of the G-parameters model is lower than before because the operating point is closer to the value where it was obtained. The relative error improvement in this case is $45.8 \%$ and $40.6 \%$ for the output voltage and $57.2 \%$ and $49.2 \%$ for the input current.

In Fig. 3.15 different measures of the overall error of the models compared with the switching model are presented. It can be clearly seen that the polytopic model has the best performance both for the output voltage and the input current. The error reduction obtained using the polytopic model is around $50 \%$ for both variables.

Regarding the simulation time, in this case the switching model takes $82 \mathrm{~s}$ to complete the simulation, whereas the G-parameters, Wiener-Hammerstein, and polytopic models take $2 \mathrm{~s}, 2 \mathrm{~s}$, and $6 \mathrm{~s}$, respectively. It can be seen that as the switching frequency increases, the reduction in computational burden of the average models is more evident, compared with the switching model. 

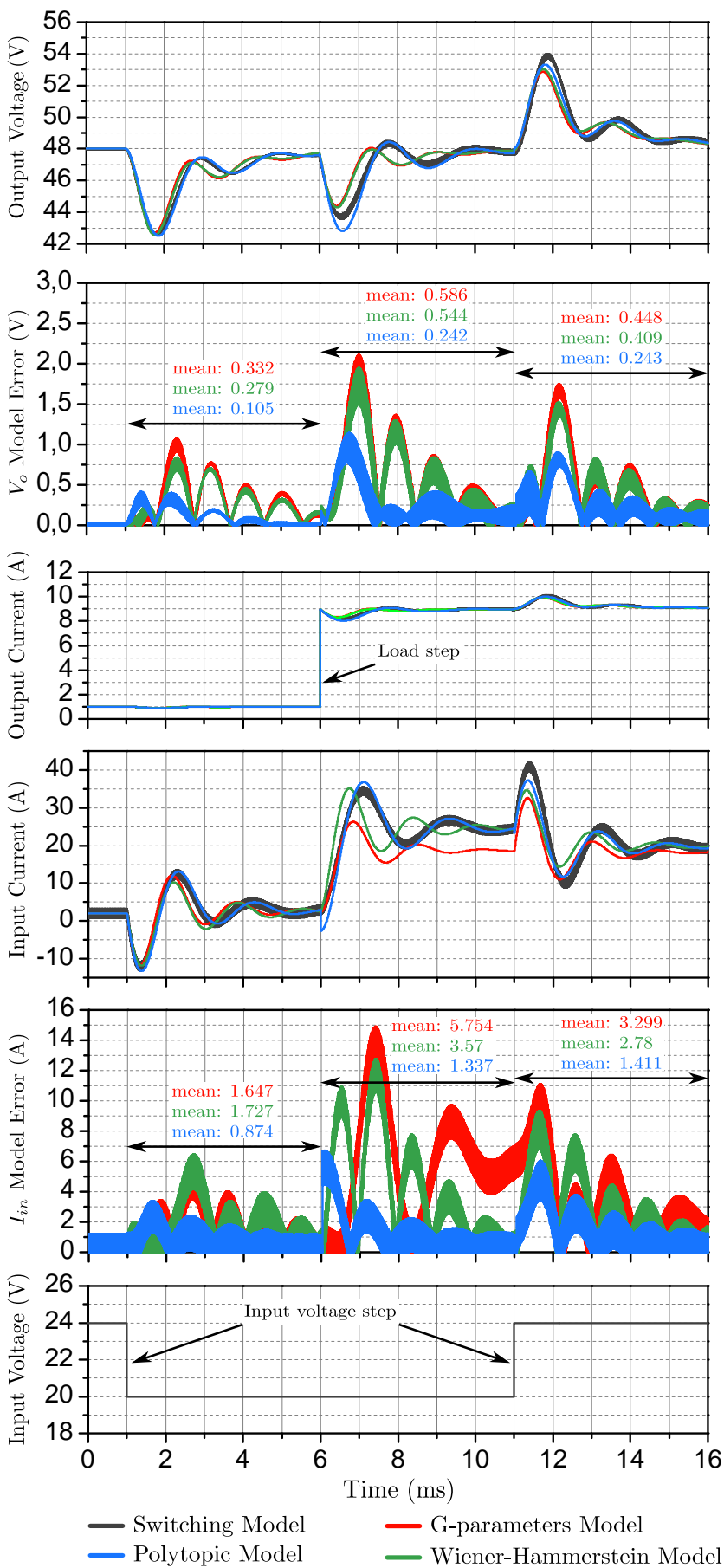

Figure 3.14: Comparison between the switching model and the three types of blackbox structures of a voltage-controlled synchronous boost converter. 

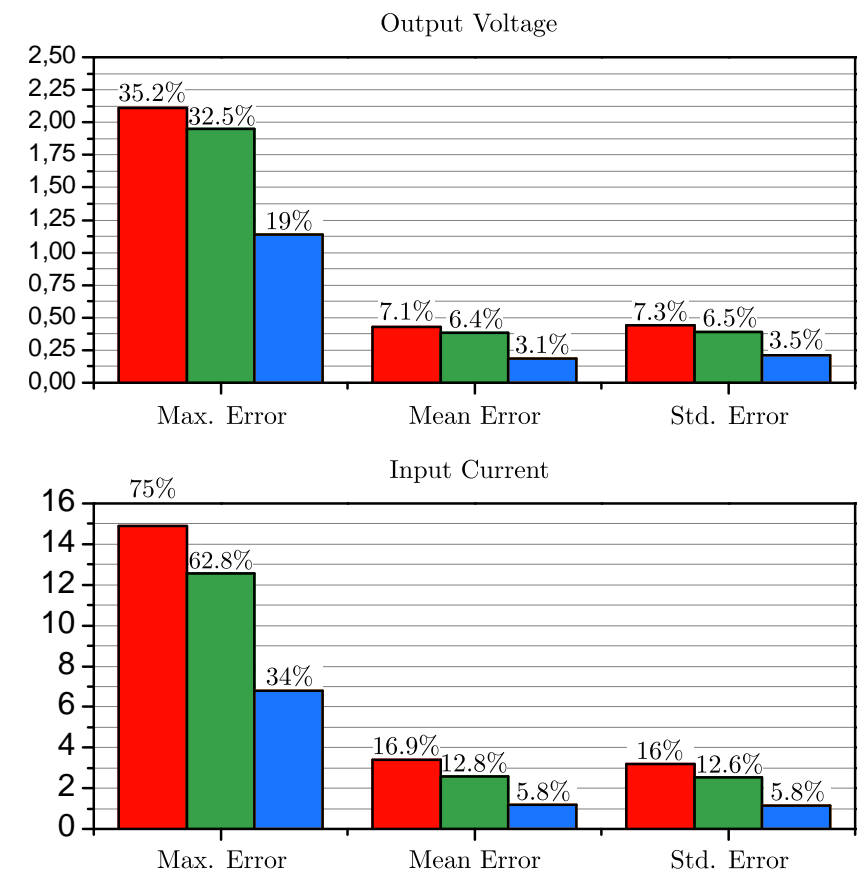

— G-parameters Model — Wiener-Hammerstein Model

- Polytopic Model

Figure 3.15: Error comparison between the switching model and the three types of blackbox structures of a voltage-controlled synchronous boost converter.

\subsubsection{Buck-boost converter case}

This part studies the dynamic behavior of the buck-boost converter in open and closed loop. The dynamic behavior of the buck-boost converters, similarly to the boost converter case, is much more dependent on the operating point than the dynamic of buck converters, as it will be shown below. As in the previous cases, the performance of the existing blackbox modeling approaches will be compared with the switching model response in order to assess their capabilities in different scenarios.

\section{Synchronous buck-boost converter in open loop}

First, the open loop characteristic of the converter is studied. Fig. 3.16 shows the scheme of the circuit implemented. As in the previous cases, the converter has been analyzed analytically in order to study the influence of the operating points on its dynamic behavior. In Appendix B.3 the SSA representation of the buck-boost converter in open loop is described and the G-parameters are derived. In this case, the duty cycle appears in the state matrix, hence the placement of the poles will depend on its value. The duty cycle also appears in the input and output matrices, but in this case multiplied by the parasitic resistance of the output capacitor, so it is expected to affect less the zeros of the transfer functions. This conclusions can be confirmed by checking the analytical G-parameters transfer functions ((B.23) and (B.24)), where the strong dependency of the natural frequency and the damping factor with the duty cycle is explicitly exposed. Besides the duty cycle also affects considerably the gain of the audiosusceptibility and the buck current gain. In Fig.B.6 the bode plots of the G-parameters models for different values of the duty cycle are presented.

The analytical G-parameters model has been evaluated with the parameters shown in Fig. 3.16 and its dynamic response to steps in the input variables has been compared with the switching model. Fig. 3.17 shows the response of both models. At time $t=1 \mathrm{~ms}$ a 


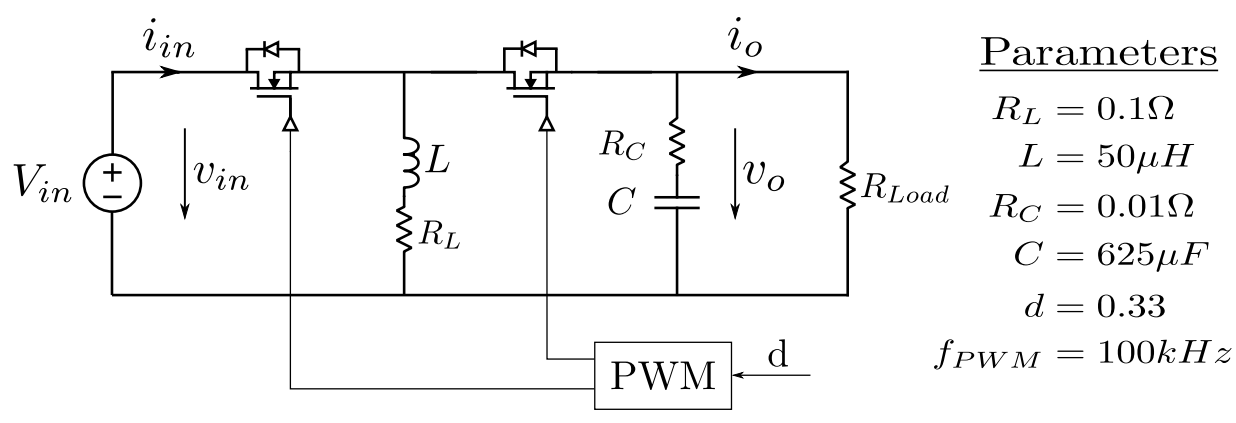

Figure 3.16: Synchronous buck-boost converter circuit.
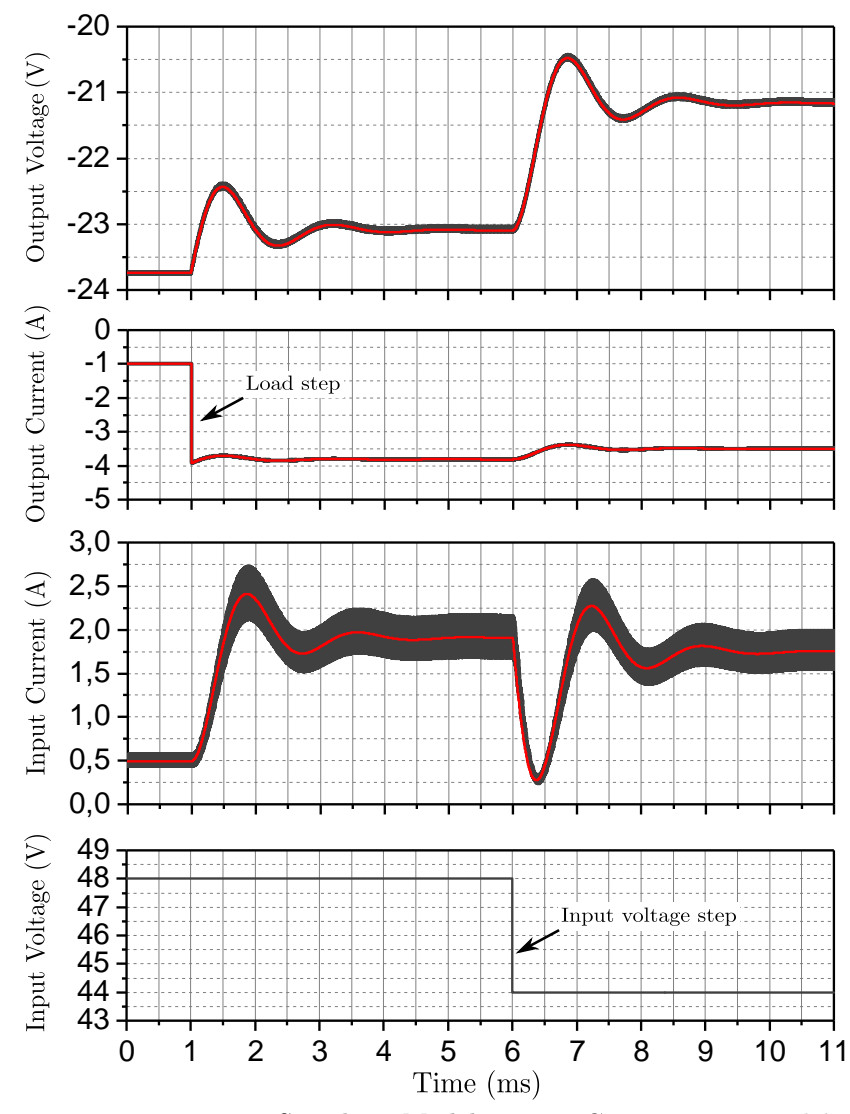

— Switching Model — G-parameters Model

Figure 3.17: Comparison between the switching model and the G-parameters model of a synchronous buck-boost converter in open loop.

resistor is added in parallel with the load, increasing the output current from $I_{o}=-1 \mathrm{~A}$ to $I_{o}=-4 \mathrm{~A}$, whereas at time $t=6 \mathrm{~ms}$ the input voltage is decreased with a step from $V_{i n}=48 \mathrm{~V}$ to $V_{i n}=44 \mathrm{~V}$. It can be seen how the G-parameters model represents the behavior of the converter neatly.

As in the cases of the buck and boost converters in open loop, the nonlinearities in the system comes from the product of the duty cycle with the variables of the converter. In the open-loop simulations the duty cycle is constant, therefore the equations become linear and the dynamic behavior of the converters can be represented with transfer functions. 


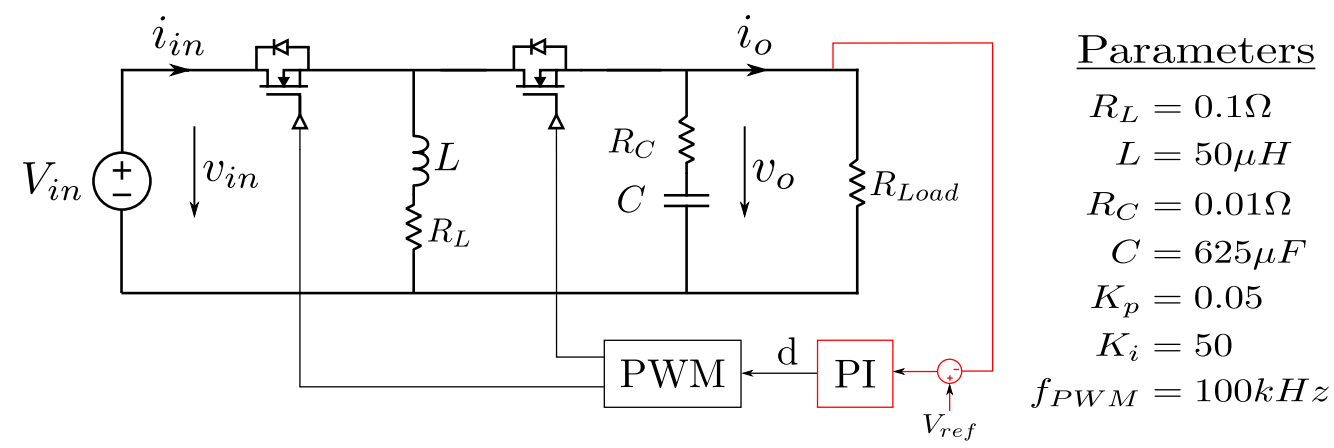

Figure 3.18: Voltage-controlled synchronous buck-boost converter circuit.

Table 3.4: Parameters of the Wiener-Hammerstein model of the voltage-controlled synchronous buck-boost converter.

\begin{tabular}{|c|c||c|c||c|c|}
\hline \multicolumn{2}{|c||}{ Output filter } & \multicolumn{2}{c||}{ Input filter } & \multicolumn{2}{c|}{ Audiosuscentibility } \\
\hline \hline$L_{\text {out }}$ & $24.2 \mu \mathrm{H}$ & $L_{\text {in }}$ & $995.3 \mu \mathrm{H}$ & $L_{G}$ & $166.3 \mu \mathrm{H}$ \\
\hline$R_{L}$ & $3.3 \Omega$ & $R_{\text {in }}$ & $1.96 \Omega$ & $R_{G}$ & $0.13 \Omega$ \\
\hline$C_{\text {out }}$ & $538.3 \mu \mathrm{F}$ & $C_{\text {in }}$ & $15.1 \mu \mathrm{F}$ & $C_{G}$ & $17.8 \mathrm{~F}$ \\
\hline$R_{C}$ & $26.8 \mathrm{~m} \Omega$ & & & & \\
\hline
\end{tabular}

\section{Voltage-controlled synchronous buck-boost converter}

The next step is to close the loop, so the duty cycle varies and the system becomes nonlinear. Fig. 3.18 shows the scheme of the voltage-controlled synchronous buck-boost converter regulated with a PI controller. In Appendix C.3 the SSA representation of the converter is derived. In (C.13) it can be seen that the state matrix is affected by the two inputs of the G-parameters model, so the operating point is expected to affect the placement of the poles of the system. Also, the input and output matrices depend on the operating point of the input of the model, therefore also the placement of the zeros will be affected by the operating point. In Fig. C.6 the bode plot of the G-parameters obtained around different operating points are represented.

The G-parameters models used in the comparison will be obtained using the analytical equations by changing the linearization point. Different operating points of the two inputs are considered. In particular the points selected are the nine combinations of $V_{\text {in }}=\{40,44,48\} V$ and $I_{\text {out }}=\{-1,-5.5,-10\} A$. As small-signal model, the G-parameters model linearized around $V_{\text {in }}=40 \mathrm{~V}$ and $I_{\text {out }}=-1 \mathrm{~A}$ is used. The nine small-signal models are integrated in a polytopic structure, using the middle points between operating points as center of the weighting functions and a slope of 10 for both variables (see (2.19) and (2.20)). The parameters of the transfer functions are shown in Table C.5 and in Table C.6, which have been obtained evaluating the analytical equations with the parameters given in Fig. 3.18 and linearized around the operating points mentioned before. However, the Wiener-Hammerstein model was obtained using identification techniques from the response of the converter to steps in the input voltage and the output current. The structure of the model is the one shown in Fig. 2.10. The parameters obtained in the identification are shown in Table 3.4.

Figure 3.19 shows the comparison between the switching model and the three models obtained. The initial operating point is $V_{i n}=48 \mathrm{~V}$ and $I_{o}=-1 \mathrm{~A}$. At time $t=1 \mathrm{~ms}$ the input voltage has a negative step to $V_{i n}=40 \mathrm{~V}$, which is the operating point where the 
G-parameters model was identified. It can be seen that none of the models are able to reproduce the transient behavior accurately. The G-parameters model has an initial higher overshoot, however in the final part of the transition the switching model approaches the behavior of this model. The Wiener-Hammerstein model has a lower overshoot and higher resonant frequency. Finally, the polytopic model has the same resonant frequency but a higher amplitude of the oscillations.

At time $t=6 \mathrm{~ms}$ a resistor is added in parallel with the load, increasing the output current demand to $I_{o}=-10 \mathrm{~A}$. In this case the polytopic model has the best accuracy, even though the initial part of the transitory has a slight lower overshoot and frequency than the switching model. As the operating point where the G-parameters and Wiener-Hammerstein model is now further away from the one in which the converter is working, the dynamic performance of these models start to be compromised, having considerable differences in frequency and amplitude of the response. It can be also seen that the estimation of the steady state value of the input current given by the G-parameters model is lower than the real one, whereas the Wiener-Hammerstein model is able to track the nonlinearity of the static part of this variable.

Finally, at time $t=11 \mathrm{~ms}$ the input voltage is restored to $V_{i n}=48 \mathrm{~V}$. In this case the initial part of the response of the polytopic model suffers a discontinuity both in the output voltage and the input current. Therefore, the initial performance of the model is poor, but it improves at the final part of the transitory response. The performance of the G-parameters and Wiener-Hammerstein models is better than in the previous step, because the operating point is closer to their nominal one, but still there are differences in the amplitude of the oscillations.

The analysis of the different measures of the overall error of the models compared with the switching model is presented in Fig. 3.20. In this case there is not a clear conclusion. For instance, the lower maximum error for the output voltage is obtained with the G-parameters model, whereas the polytopic model has the lower mean error and standard deviation. Regarding the input current, the lower error is obtained with the Wiener-Hammerstein model, however the differences among the models are not substantial.

However, comparing the characteristics of the transient responses, such as the overshoot, the settling time, frequency of the oscillations, etc. the polytopic model is the one that represents better the behavior of the converter, as it can be seen in Fig. 3.19. The reason why this improvement is not reflected in the error analysis is that the frequency of the oscillations in the polytopic model is slightly lower than in the switching model, which is strongly penalized when the difference between the signals is compared.

Nevertheless, as in the previous cases it can be seen that none of the models are able to track the large-signal average dynamic behavior of the converter with high accuracy. An accurate estimation of the response of the converter can be very useful to analyze the response of the interconnection of different converters, to design system-level controllers or to check the compatibility with standards. In these cases, the errors in the estimation of each converter can lead to poor overall designs. Consequently, the need for more accurate blackbox modeling approaches is again exposed.

Regarding the computational burden, the switching model takes $103 \mathrm{~s}$ to complete the simulation shown before, whereas the G-parameters, Wiener-Hammerstein, and polytopic models take $3 \mathrm{~s}, 3 \mathrm{~s}$, and $8 \mathrm{~s}$, respectively. As mentioned before, the integration time for the switching model must be higher than the switching frequency, in this case the minimum integration time was $10 \mathrm{~ns}$. On the other hand, the integration time of the blackbox models, as they only capture the average response, can be reduced to $1 \mu \mathrm{s}$, resulting in a substantial reduction in the computational burden. 

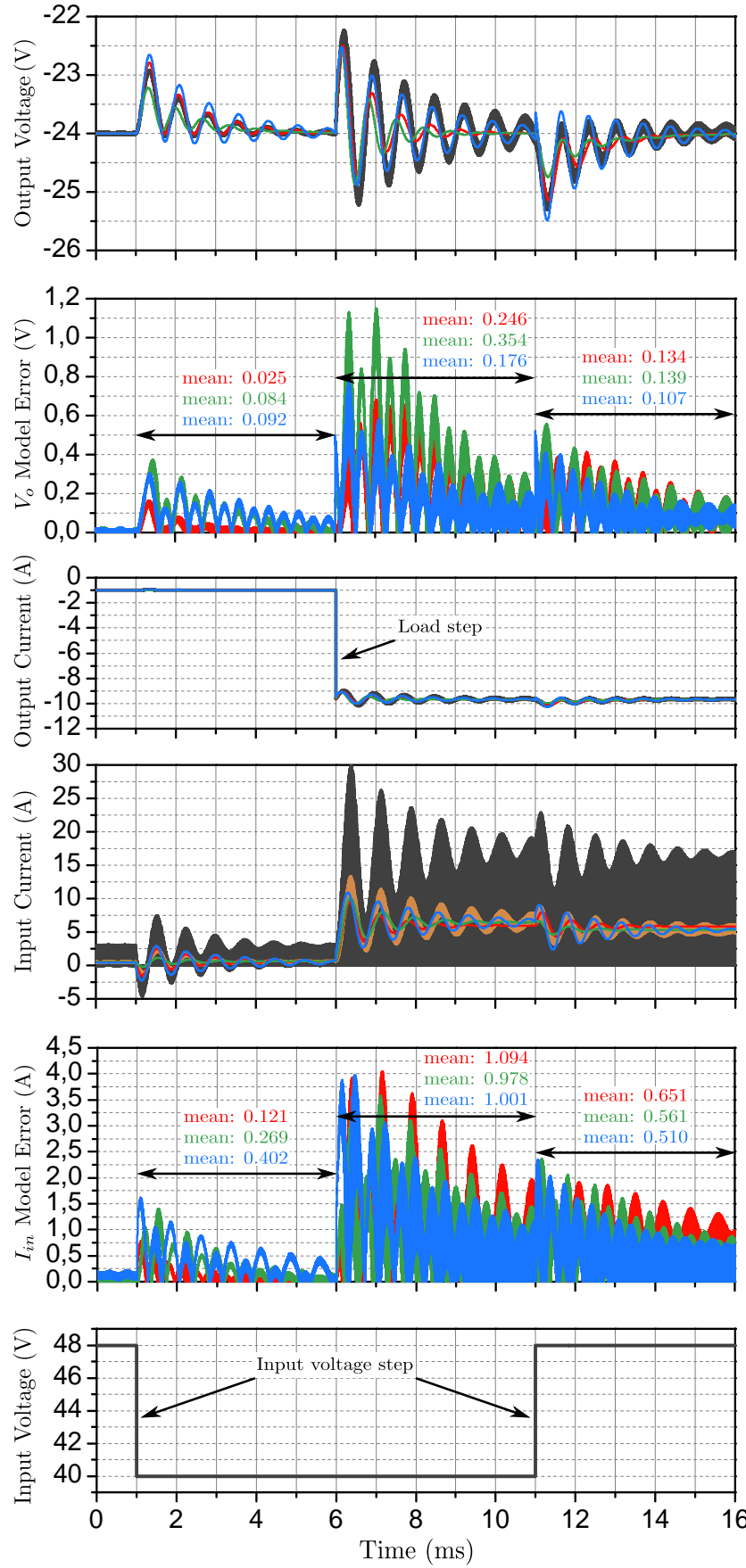

$$
\begin{array}{ll}
\text { — Switching Model } & \text { - Switching Model (Filtered) } \\
\text { — G-parameters Model } & \text { - Wiener-Hammerstein Model } \\
\text { — Polytopic Model } &
\end{array}
$$

Figure 3.19: Comparison between the switching model and the three types of blackbox structures of a voltage-controlled synchronous buck-boost converter. 

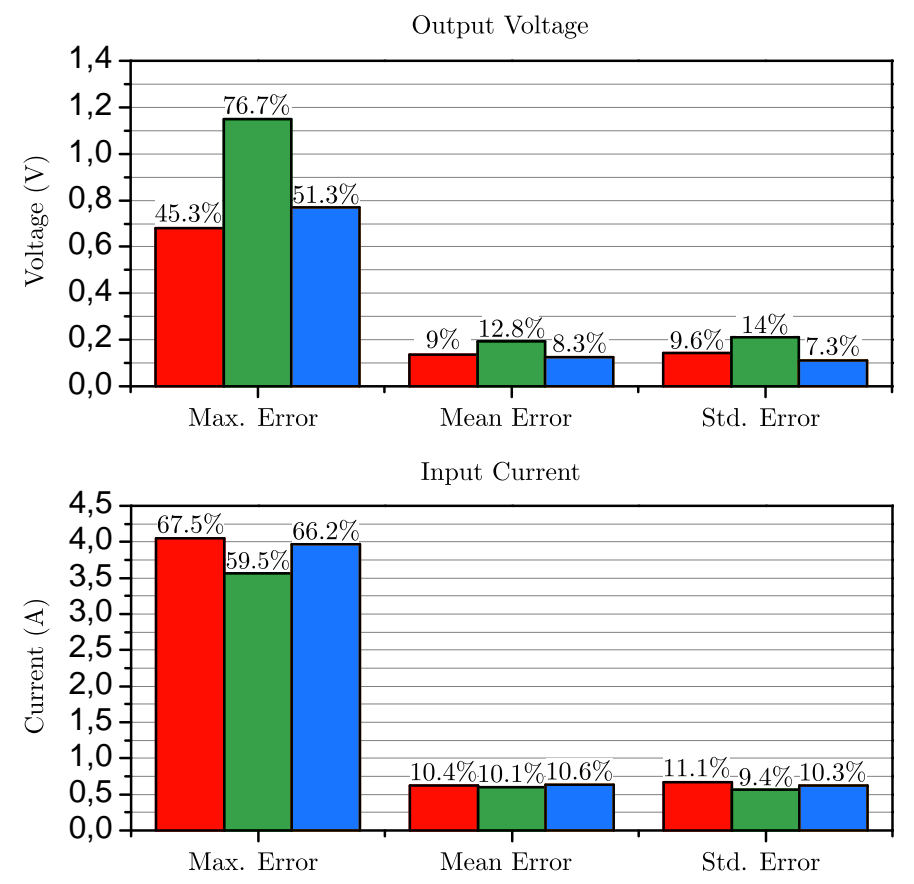

— G-parameters Model — Wiener-Hammerstein Model

- Polytopic Model

Figure 3.20: Error comparison between the switching model and the three types of blackbox structures of a voltage-controlled synchronous buck-boost converter.

\subsection{Converters with different operation modes}

In Section 2.1.2 the DBS control was introduced. It was explained how a strategy to avoid overloading the microgrid is to define certain regions in the bus voltage where the converters change their operation mode. As the converters regulating the bus voltage use droop control, the bus level depends on the power flow in the microgrid. If the power delivered by the sources (in general renewable sources working with MPPT control) is higher than the power that the microgrid can consume or storage, the bus voltage will increase due to the droop control strategy. When the voltage is at the level defined as maximum, the sources will change their operating mode from delivering maximum power to regulate the bus voltage, thus providing just the power that can be handled by the microgrid. Another example of change in the mode of operation of converters working in dc microgrids is when the converter is working in voltage mode and it reaches its maximum current level, in this case the converter will stop working in voltage mode and start working in current mode, providing maximum current.

In the following the latter case will be studied. Fig. 3.21 depicts a synchronous buck converter with a double-loop control. The inner loop regulates the inductor current with a PI controller. The current reference is given by the outer loop, which regulates the output voltage with another PI controller. Besides, in order to protect the converter, the current reference is limited to a determined value.

The focus of the analysis will be put in the performance of the different modeling approaches to account for the dynamic response of the converter in voltage and current mode, as well as during the transition from the voltage to current mode due to the current limitation. Consequently, the response of the converter to load steps will be studied. The G-parameters that represent the response of the output voltage and the input current when the output current is perturbed, $Z(s)$ and $H(s)$ respectively, are obtained in voltage and 


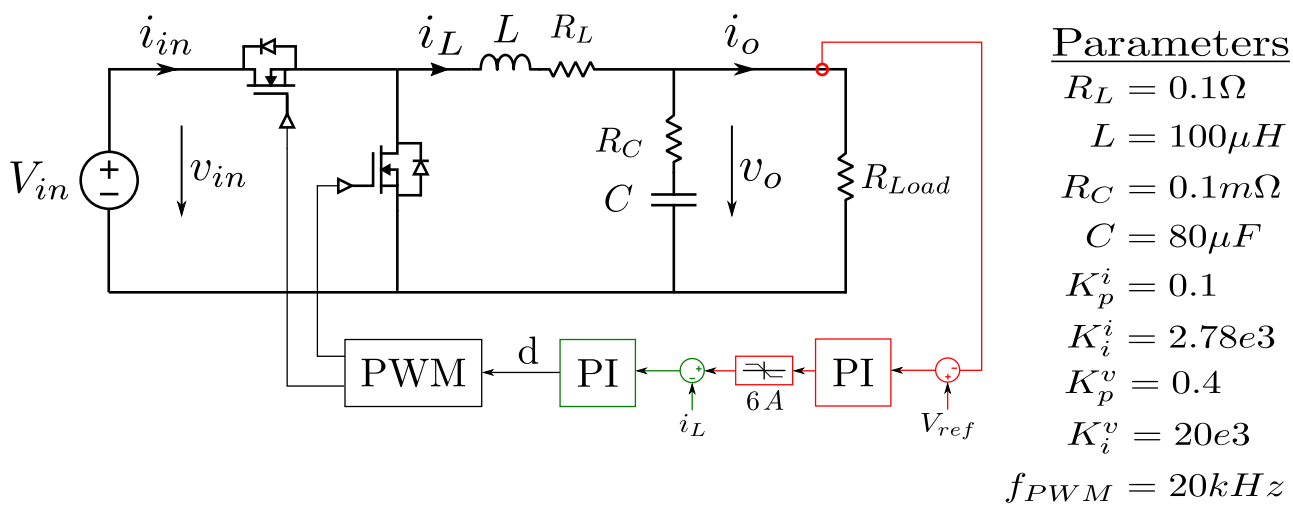

Figure 3.21: Voltage-controlled synchronous buck converter circuit with a double-loop control.

Table 3.5: Parameters of the identified transfer functions of the voltage-controlled buck converter with double-loop control in the two different modes of operation.

\begin{tabular}{|c||c||c|}
\hline Operation mode & Output impedance, Z(s) & Back current gain, H(s) \\
\hline \hline Voltage mode & $\frac{-1.25 e 4 s+6.34 e 4}{s^{2}+5084.7 s+2.52 e 8}$ & $\frac{2613.2 s+6.52 e 7}{s^{2}+5362.8 s+2.53 e 8}$ \\
\hline Current mode & $\frac{-11300.1}{s}$ & $\frac{0.054 s-1411.5}{s}$ \\
\hline
\end{tabular}

current mode. The transfer functions identified are shown in Table 3.5 and compared with the frequency response of the converter in Fig. 3.22. The operating point selected for the voltage and current mode was $I_{\text {out }}=3 \mathrm{~A}$ and $I_{\text {out }}=6.1 \mathrm{~A}$, respectively, and the amplitude of the perturbation was $0.1 A$ in both cases.

Regarding the Wiener-Hammerstein model, the parameters of the output filter were identified in time domain, using the response of the output voltage to a step in the output current. In order to account for the current mode, the combination of a voltage source, a MOSFET, and a current source is proposed, as depicted in Fig. 3.23. While the MOSFET is $\mathrm{ON}$, the voltage at the left side of the inductor is fixed to the voltage reference, so the current source does not affect the circuit. When the output current is higher than the maximum value, the MOSFET is turned OFF, but the voltage at the left of the inductor will be still fixed to the voltage reference until the inductor current reaches the maximum current value, because the difference is circulating through the diode of the MOSFET and, therefore, fixing the voltage. Once the inductor current reaches the maximum current value, the current reference will be the only one affecting the circuit. For the transition from current limitation to voltage mode, two conditions are imposed: the output current should be lower than the maximum value and the output voltage should be higher than the reference. The latter condition is included because if the voltage is lower than the voltage reference, there will be a voltage restoration process that will keep the current reference in the voltage loop in the maximum value.

Finally, the polytopic model is composed of the two G-parameters models obtained in voltage and current mode. A weighting function does not make sense in this kind of transition, because it is a sharp transition between two modes of operation with very 
Output Impedance $(\mathrm{Z})$
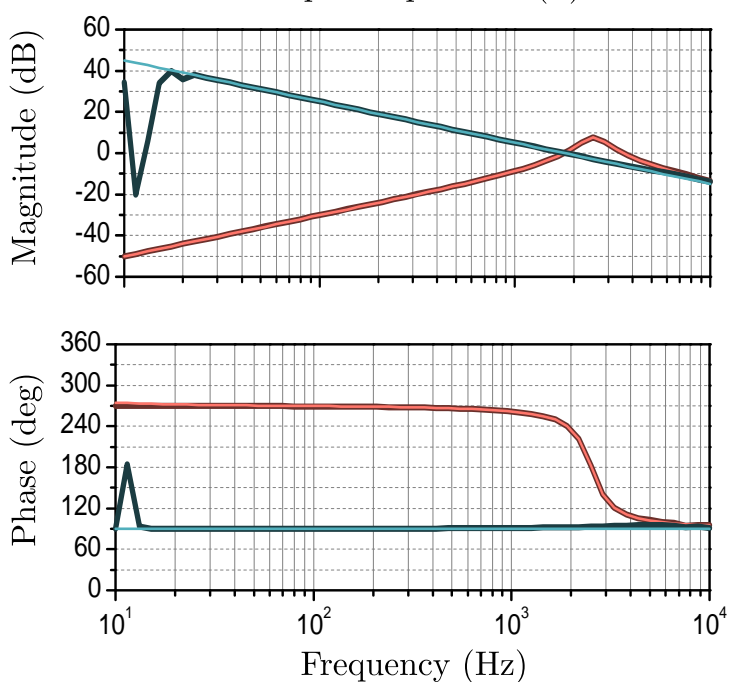

Back Current Gain (H)
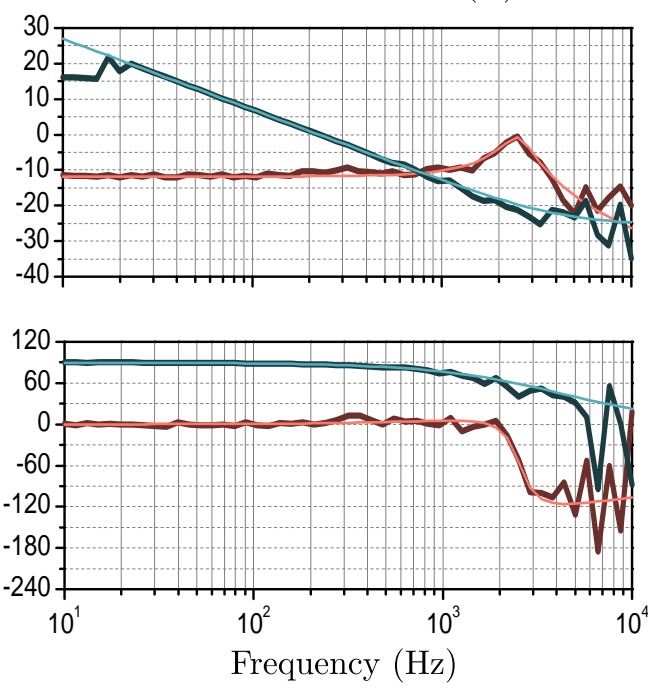

— Switching model (VM) Identified model (VM)

- Switching model $(\mathrm{CM})$ Identified model $(\mathrm{CM})$

Figure 3.22: Frequency response of the voltage-controlled buck converter with double-loop control in voltage mode (VM) and in current mode (CM). Comparison between the measured signal and the identified transfer functions.

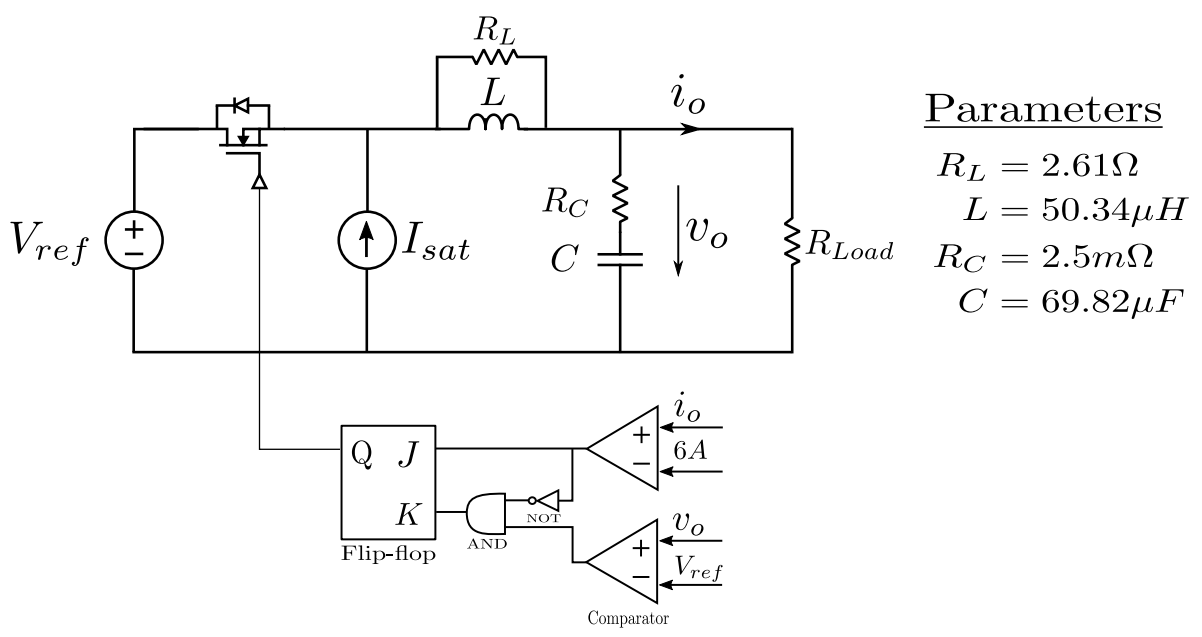

Figure 3.23: Proposed Wiener-Hammerstein model for voltage-controlled converters with double-loop control and current limitation. 

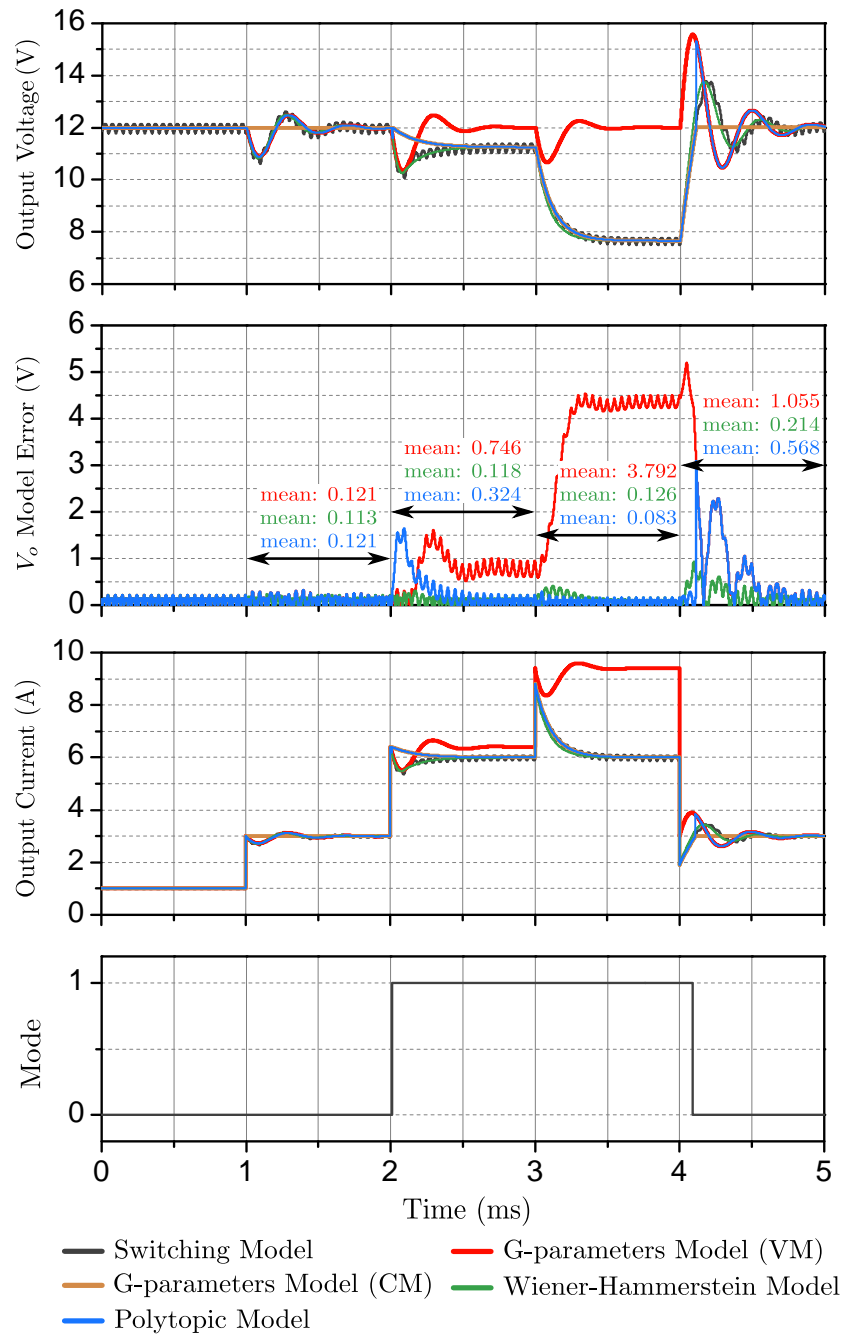

Figure 3.24: Comparison between switching model and the three types of blackbox structures of a voltagecontrolled synchronous buck converter with a double-loop control.

different behaviors. Therefore, the same conditions used to turn ON and OFF the MOSFET in the Wiener-Hammerstein model, are used to select what small-signal model is used in the polytopic model.

The comparison among the switching model and the different modeling structures is shown in Fig. 3.24. At time $t=1 \mathrm{~ms}$ a resistor is added in parallel with the load increasing the output current from the initial value $I_{\text {out }}=1 \mathrm{~A}$ to $I_{\text {out }}=3 \mathrm{~A}$. The G-parameters model identified in voltage mode, the Wiener-Hammerstein and the polytopic models reproduce the dynamic behavior of the converter correctly, whereas the G-parameters model in current mode is not active because it would have a high value of output voltage. At time $t=2 \mathrm{~ms}$ another resistor is added increasing the output current to its limit. This load step leads the converter to work in current mode, but the transition is not as sharp as the variation of the load current. It can be seen how the converter starts with the voltage mode behavior and changes to current mode at a certain point of the transition. The Wiener-Hammerstein model with the proposed reference is able to reproduce this transition accurately, as it does not start working in current mode until the inductor current has reached the limit value. The polytopic model uses the output current to change from voltage mode to current mode, so it switches instantly to current mode and its performance is poor. 


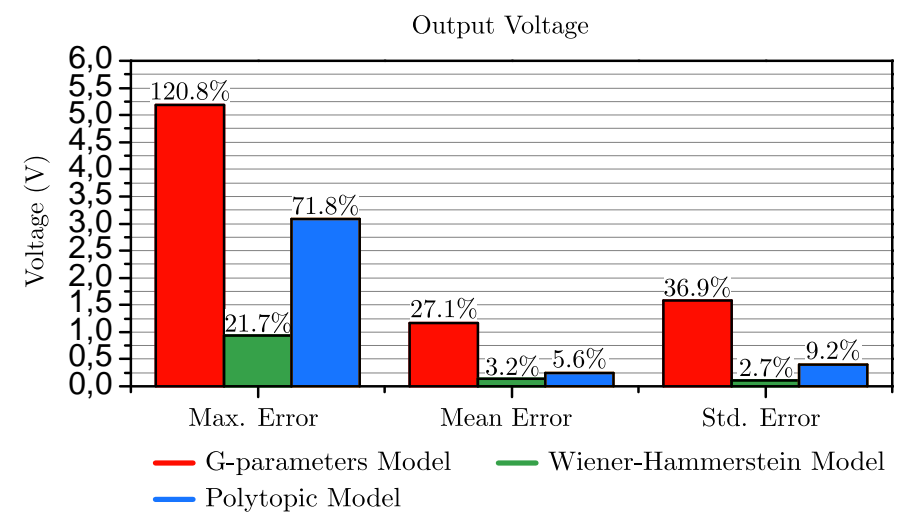

Figure 3.25: Error comparison between the switching model and the three types of blackbox structures of a voltage-controlled synchronous buck converter with a double-loop control.

At time $t=3 \mathrm{~ms}$ another resistor is added to the load, but as it is in current mode, the load step leads to a reduction of the output voltage. The G-parameters model identified in current mode follows the dynamic response of the converter precisely, as well as the Wiener-Hammerstein and polytopic model. Finally, at time $t=4 \mathrm{~ms}$ the last two resistors are disconnected and the output current goes back to $I_{\text {out }}=3 \mathrm{~A}$. The change in the output current is sharp, due to the use of resistors, but the internal reference of current is not reduced until the output voltage is restored to the nominal value. This effect leads the converter to have a current mode behavior until the output voltage is restored. This condition has been added to the switching between modes of the models, as shown in Fig. 3.23. The Wiener-Hammerstein model with the proposed reference follows the behavior of the converter with high accuracy, whereas the polytopic model only captures the dynamic behavior while the converter is working in current mode, the transition to voltage mode is sharp and the initial conditions are different, so the result is again poor.

In Fig. 3.25 different measures of the error between the switching model and the blackbox models are presented. It can be clearly seen that the Wiener-Hammerstein model with the proposed reference has a superior performance compared with the other two approaches.

\subsection{Conclusions}

In this chapter the three main blackbox modeling structures have been compared. Some illustrative examples have been selected to draw the following conclusions:

- The switching nature of the electronic power converters make them time-variant nonlinear systems. Analytically, it is possible to obtain a time-invariant model by describing the state-space equations in each of the switch configurations and weighting them by means of the duty cycle. This process generates a time-invariant nonlinear model, where the duty cycle can be found multiplying the variables of the system. If the duty cycle is constant, the switching nature of the converter will not lead to nonlinearities. However, in regulated converters the duty cycle is also a variable in the system that depends on the operating point in which the converter is working. The equations can be linearized around an operating point in order to obtain an LTI model.

- It is also possible to obtain a LTI model of the converter using a blackbox approach. The process consists on making the converter work in a specific operating point and perturbing the inputs of the model (in time or frequency domain). If the perturbation is high enough to excite the output variables of the converter so that its response is 
high compared with the switching ripple, but small enough so it does not show a nonlinear behavior, the response of the converter can be used to identify transfer functions that describe the behavior of the converter. This process is the equivalent to linearize the nonlinear equations around an operating point.

- The accuracy of the small-signal models, obtained either analytically or using identification methods, will depend on the proximity of the operating conditions to the operating point where the model was linearized. It also depends on how the dynamic of the converter changes for different values of the duty cycle, or the operating point in case of regulated converters. In this chapter, the three main non-isolated topologies of electronic power converters, i.e. buck, boost, and buck-boost, has been studied. In Appendix B the analytical expression of the G-parameters model of these converters were derived, whereas in Appendix $\mathrm{C}$ the state-space representation of these converters with a PI regulator was presented. These expressions show that the dynamic behavior of the buck converter has a much lower dependency on the operating point than boost and buck-boost converters. These facts are presented with some examples in this chapter.

- The polytopic model is a weighted combination of linearized models around different operating points. Therefore, it is able to follow accurately the dynamic response of the converter to small perturbation around any operating point. However, its performance to a large perturbation is not always satisfactory. In this chapter it has been shown how the accuracy of the model is lower during the initial part of the transitory, whereas it is better at its final part. This effect will be studied in the next chapter.

- The Wiener-Hammerstein model consist of linear networks that account for the dynamic of the converter and static references that can be nonlinear to adapt the static behavior of the model to possible nonlinearities in the converter. Due to its structure, it is not able to capture the changes in the dynamic behavior of the converter due to variation in the operating point. However, it is able to account for the static nonlinear behavior of the input current in voltage-controlled converters.

- A well-known strong nonlinearity in electronic power converters is the discontinuous conduction mode. It appears in certain converters when the inductor current reaches zero during a switching cycle and it cannot flow in the negative direction. In this mode of operation, the dynamic of the converter depends on the amount of time that the inductor current is zero, which depends on the load demand. In this chapter it has been shown that it is possible to obtain small-signal models around different values of the output current that define the behavior of the converter accurately around these operating points. Besides, the large variation of the frequency response of the converter for different values of the output current has been also presented. If only one of the small-signal models is used, the performance of the model is very poor when the output current is variable. The Wiener-Hammerstein model is not able to account for nonlinearities in the dynamic behavior of the converter, so it is not considered as an option for this kind of converters. The polytopic model is able to account for small perturbation around different values of the output current, however its performance for large load steps or transitions from discontinuous to continuous conduction modes is also poor. A strategy to improve the accuracy of the model for this kind of nonlinearities will be proposed in the next chapter.

- Another common source of dynamic nonlinearities in electronic power converters in dc microgrids are changes in the operation mode of the converters. In this chapter the effect of a current limitation included in the control strategy of a converter is studied. The converter is controlled with a double-loop control, where the inner loop regulates the inductor current and the outer loop regulates the output voltage 
by setting the reference current to the inner loop. It has been shown how it is possible to obtain a small-signal model in both voltage and current mode, which have very different dynamic behaviors. The polytopic model integrates this two models and the conditions to switch from voltage mode to current mode is just that the output current is higher that the limit value. However, the conditions to change from current mode to voltage mode is not only that the output current is lower than the limit value but also that the output voltage is higher than the nominal value, in order to account for the voltage restoration, which keeps the current reference to the limit value. Regarding the Wiener-Hammerstein model, a circuit is proposed for the reference of the output network, which integrates a voltage source, a MOSFET, and a current source. This approach is able to account for the dynamic behavior of the inductor current and, hence, it is able to follow accurately the transitions from voltage to current mode and vice-versa. On the other hand, the polytopic model is able to represent well the behavior of the converter in both modes of operation, but during the transitions between modes its performance is unsatisfactory. These phenomena will be also studied in the next chapter.

The comparison among the existing blackbox modeling techniques in different scenarios, highlighting their advantages and disadvantages, as well as their capabilities and limitations, is one of the contributions of this thesis. 


\title{
Polytopic model with dynamic weighting functions
}

\begin{abstract}
T he previous chapter presented a comparison among the different blackbox modeling structures for dc electronic power converters. Various kinds of nonlinearities were introduced for the basic power electronic topologies. Regarding the performance of the polytopic model, it was shown how it is able to reproduce neatly small perturbations around any operating point of the converters. However, in case of a large step the model starts to fail to reproduce the dynamic behavior of the system. These phenomena were presented for regulated power converters, converters with discontinuous and continuous conductions modes, and control strategies with different operation modes. In this chapter a methodology is proposed to improve the accuracy of the polytopic models when sharp and large steps occur in the input variables of the model.
\end{abstract}

\subsection{Dynamic weighting functions}

Analytical polytopic models are usually described with state space representations (2.18) [84]. This approach is very convenient because the nonlinearities can be function of state or input variables. However, using a blackbox approach the internal architecture of the systems is unknown, therefore the only variables available are the input and output variables. This is the reason why blackbox polytopic models are defined for input-output representations (see Fig. 2.11) [106]. In case the nonlinearities are function of the state variables, the steady-state value can be related with the input variables of the system, however their dynamic behavior will be generally different.

Regarding dc electronic power converters, the inputs of the system, i.e. the input voltage and output current in the case of the G-parameters model (see Fig. 2.6), can vary sharply, but the state variables, which are defined by their first derivative, will have a dynamic behavior related with the eigenvalues of the state matrix. As the blackbox polytopic model uses the input variables as inputs of the weighting functions, when a sharp step in the input variables occurs the model will change its dynamic behavior also sharply from the initial local model to the one corresponding to the final operating point. On the other hand, the system is actually modifying its dynamic behavior according to the state variables, which do not change sharply. This is the reason why in the previous chapter, the polytopic model was inaccurate at the initial part of the transitory responses, whereas at its final part 
the similarity improved. Consequently, it is reasonable to think that the accuracy of the polytopic model could be improved by defining the transition among the small-signal models in relation with the dynamic of the state variables. Mathematically, the state variables can be related with the input variables as follows:

$$
\begin{aligned}
\dot{x} & =A x+B u \\
s x & =A x+B u \\
(s I-A) x & =B u \\
x & =(s I-A)^{-1} B u
\end{aligned}
$$

or equivalently:

$$
x=\frac{1}{\operatorname{det}(s I-A)} \operatorname{adj}(s I-A) B u
$$

where $\operatorname{adj}(\cdot)$ represents the adjugate and $\operatorname{det}(\cdot)$ represents the determinant of a matrix. It can be seen how the matrix of transfer functions that relate the input variables with the state variables will have a common denominator, defined by the eigenvalues of the system, and different numerators, as a result of the product of the adjugate of the matrix $(s I-A)$ and the input matrix. The same denominator can be found in the relationship between the output and input variables, which are the transfer functions identified in the small-signal model (see (B.5)).

According to this analysis a possible method to improve the accuracy of the polytopic models is using dynamic weighting functions instead of the classical static ones. The idea is to add a transfer function between the input variables and the static weighting functions such that the dynamic transition among small-signal models is limited by the internal dynamic of the system, but the steady state remains unchanged, as by definition the local models give the best approximation around the operating point where they were identified.

The proposed transfer function will have the form:

$$
w(u(s), s)=w_{s}(u(s) \tau(s))
$$

where $w$ is the dynamic weighting function, $w_{s}$ is the classical static weighting function (see 2.19), $u(s)$ is the input variable, and $\tau(s)$ is a transfer function with the characteristics mentioned above, that can be defined as follows:

$$
\tau(s)=\frac{a_{0}}{\operatorname{det}(s I-A)}=\frac{a_{0}}{s^{n}+a_{n-1} s^{n-1}+\ldots+a_{1} s+a_{0}}
$$

where $a_{n-1}, \ldots, a_{1}, a_{0}$ are the parameters of the characteristic polynomial, which are already available in the poles of the identified transfer functions. The numerator is equal to $a_{0}$ in order to make the steady state value of the transfer function unitary, so it does not affect the static response of the model. This transfer function can be seen as the characteristic filter of the system, as it is based on the characteristic polynomial. It would be also possible to use just one of the poles of the system, approximate the dynamic of the characteristic filter with a first order transfer function, or use the natural frequency in case of complex poles.

It is important to have in mind the conditions that the weighting functions must satisfy, i.e. their value must be between 0 and 1 and their sum has to be always equal to 1 


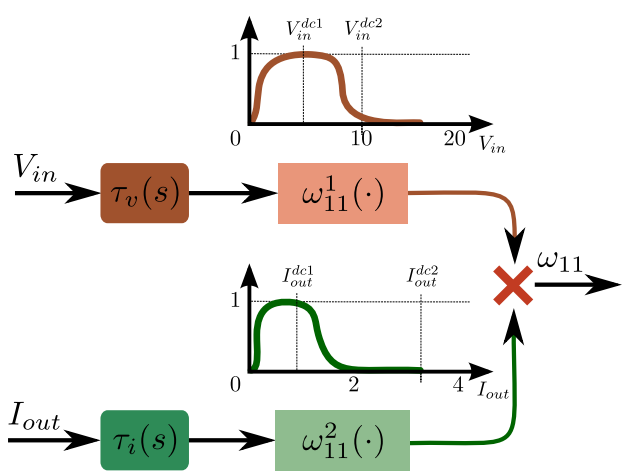

Figure 4.1: Scheme of the dynamic weighting function of one operating point defined by the input voltage and the output current.

(2.21). This fact imposes that all the static weighting functions related to one variable must have the same input signal. However, it is possible to use a different transfer function for every variable considered in the weighting functions, e.g. use one pole for a variable and a different pole for another variable. Using this approach the flexibility of the model is increased and, furthermore, the design of the dynamic weighting functions can be performed in a deterministic way, i.e. a limited and well-defined number of options can be implemented and compared with the behavior of the converter, so using a figure of merit as the mean squared error the best alternative can be selected. A scheme of the dynamic weighting functions with input voltage and output current as input variables is depicted in Fig. 4.1.

The examples of the previous chapter, where none of the existing blackbox modeling approaches could represent accurately the response of the electronic power converter, will be recovered in this chapter. The performance of the polytopic model with the classical static weighting functions and the proposed dynamic weighting functions will be compared for these cases.

\subsection{Voltage-controlled synchronous boost converter case}

The previous chapter showed that the voltage-controlled synchronous boost converter has a variable dynamic behavior depending on its operating point. The G-parameters and the Wiener-Hammerstein models use linear models to account the dynamic behavior of the converter, so they cannot account for this phenomenon. The polytopic model is able to adapt its dynamic response according to the operating point. However, it was shown that when a large step in the input variables occurs, the performance of the model at the initial part of the transitory was not accurate. In the previous section the use of dynamic weighting functions was proposed in order to approximate the rate of change in the dynamic behavior of the converter with the poles of the system. The operating points considered are all possible combinations of $V_{i n}=\{20,22,24\}$ and $I_{o}=\{1,5,9\}$. The parameters of the transfer functions obtained are presented in Table C.3 and Table C.4.

The static part of the weighting functions is the same as in the polytopic model used in the previous chapter (Section 3.1.2), i.e. the centers of the sigmoids are set in the middle of the operating points considered and the slope is 4 for the input voltage and the output current. The transfer function that provides dynamic to the weighting functions has been designed as described before, using the equation (4.4). The poles of the initial operating point, $V_{i n}=20 \mathrm{~V}$ and $I_{o}=1 \mathrm{~A}$, was selected and the resulting transfer function is:

$$
\tau(s)=\frac{624.47 e 7}{s^{3}+2082.72 s^{2}+116.67 e 5 s+624.47 e 7}
$$


The same transfer function is used for both the input voltage and the output current.

In Fig. 4.2 the response of the switching model of the voltage-controlled boost converter to steps in the input voltage and in the load is compared with the polytopic models with static and dynamic weighting functions. At time $t=1 \mathrm{~ms}$ a step in the input voltage occurs, reducing its value from $V i n=24 \mathrm{~V}$ to $\operatorname{Vin}=20 \mathrm{~V}$. The response of the polytopic model with static weighting functions is reasonably accurate, so only a modest improvement can be seen in the model using dynamic weighting functions. However, it is interesting to see the effect of using the dynamic weighting functions in the transition among small-signal models. The step in the input voltage is sharp, therefore the polytopic model with static weighting functions modifies instantly its dynamic behavior between the initial point, $V_{i n}=24 \mathrm{~V}$ and $I_{o}=1 \mathrm{~A}$, to the final point, $V_{i n}=20 \mathrm{~V}$ and $I_{o}=1 \mathrm{~A}$. On the other hand, the polytopic model with dynamic weighting functions filters the step in the input voltage (green line), hence the input to its static weighting functions is not sharp but has the dynamic imposed by the poles of the system. Consequently, the dynamic of the polytopic model with dynamic weighting functions makes a transition starting in the initial operating point, $V_{\text {in }}=24 \mathrm{~V}$ and $I_{o}=1 \mathrm{~A}$, then activating the dynamic of the next small-signal model towards the final operating point, $V_{i n}=22 V$ and $I_{o}=1 \mathrm{~A}$, and ultimately the final operating point, $V_{\text {in }}=20 \mathrm{~V}$ and $I_{o}=1 \mathrm{~A}$.

At time $t=6 \mathrm{~ms}$ a resistor is connected in parallel with the load, increasing the output current sharply from $I_{o}=1 A$ to $I_{o}=9 A$. Similarly to the previous step, the polytopic model with static weighting functions makes a sharp transition from the initial operating point, $V_{i n}=20 \mathrm{~V}$ and $I_{o}=1 \mathrm{~A}$, to the final one $V_{i n}=20 \mathrm{~V}$ and $I_{o}=9 \mathrm{~A}$, whereas the dynamic weighting functions experience a transition from the initial operating point $V_{\text {in }}=20 \mathrm{~V}$ and $I_{o}=1 \mathrm{~A}$, the next operating point towards the final one, $V_{i n}=20 \mathrm{~V}$ and $I_{O}=5 \mathrm{~A}$, and finally the last operating point, $V_{i n}=20 \mathrm{~V}$ and $I_{O}=9 \mathrm{~A}$. Regarding the performance of the models, in this case a considerable improvement can be seen in the initial part of the transition. The estimation of the voltage drop due to the load step of the polytopic model with static weighting functions is around $5 V$, whereas the actual value is $4 \mathrm{~V}$ corresponding to a mismatch of $25 \%$. Using the dynamic weighting functions the errors are contained to less than $5 \%$. Concerning the input current, the initial operating point is $I_{i n}=2.5 \mathrm{~A}$, however when the load step occurs the model with static weighting functions suddenly jumps to $I_{i n}=-2.5 \mathrm{~A}$ and then starts the transitory response towards the final value, $I_{i n}=25 \mathrm{~A}$. This undesired behavior is due to the nonlinearity in the dc gain of the back current gain (in Fig. C.4 it can be seen that the small-signal models have different dc gains for both the back current gain and the input admittance), and the sharp transition among small-signal models. The small-signal model corresponding to the final operating point, $V_{i n}=20 \mathrm{~V}$ and $I_{o}=9 \mathrm{~A}$, initially is at $-8 \mathrm{~A}$ from its nominal value. As this model has a higher dc gain than the small-signal model corresponding to the initial point, $V_{i n}=20 \mathrm{~V}$ and $I_{o}=9 \mathrm{~A}$, there is an error in the steady state value of the input current. As the polytopic model with static weighting functions change instantly to the final small-signal model, this error appears in the output of the model. Using the dynamic weighting functions, this change among small-signal models is gradual, therefore it starts from the correct dc value and reflects the transition with high accuracy.

Finally, at time $t=11 \mathrm{~ms}$ there is a step in the input voltage from $V_{\text {in }}=20 \mathrm{~V}$ to $V_{i n}=24 \mathrm{~V}$. As in the previous cases, the sharp transition of the polytopic model with static weighting functions does not reflect the dynamic behavior accurately during the first part of the transitory, having a lower estimation of the overshoot of both the output voltage and the input current. On the other hand, the use of the dynamic weighting functions allows the model to make a transition among small-signal models, which is demonstrated to be an accurate approximation of the response of the actual behavior of the electric power converter to sharp and large steps in the input variables. The error between the switching model and the two blackbox models obtained is shown in Fig. 4.3 and Fig.4.4. 

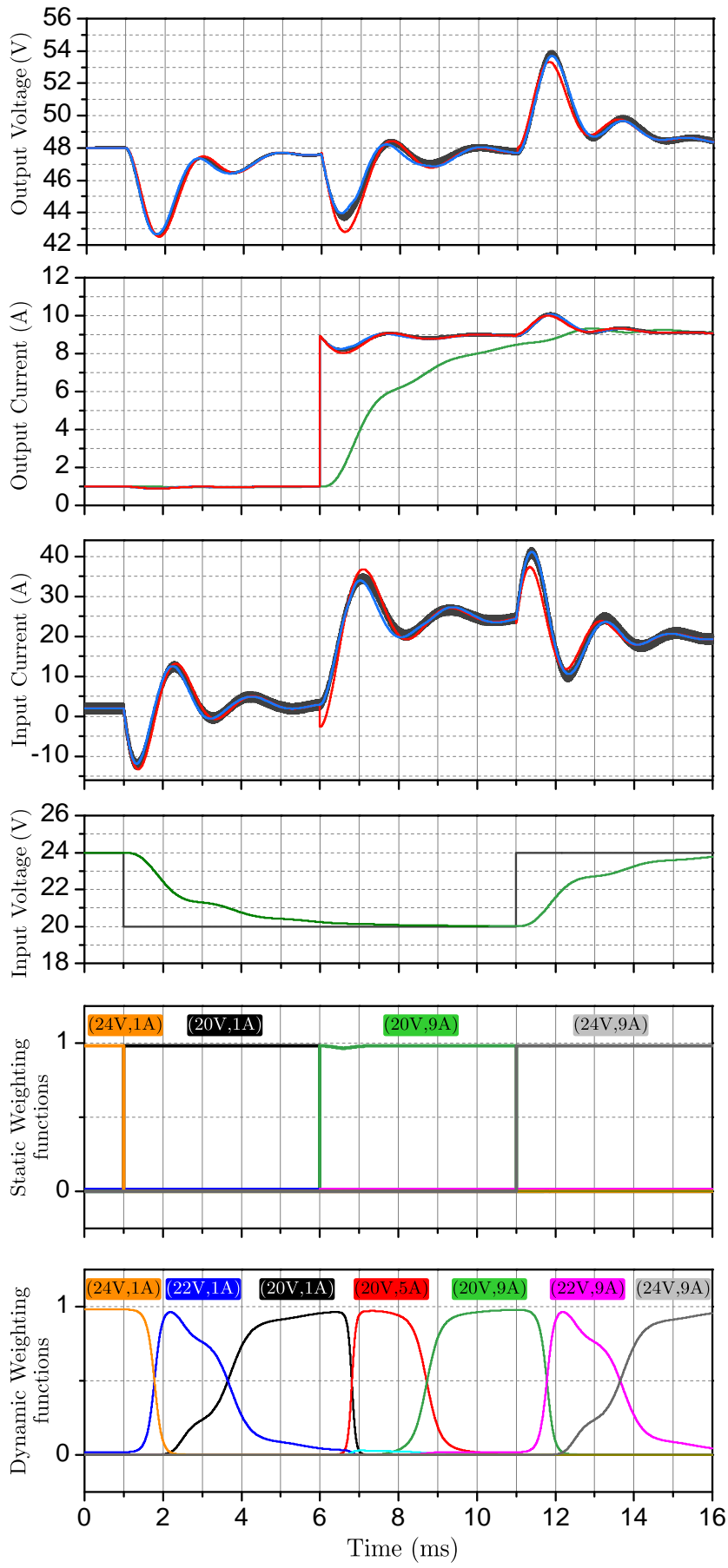

— Switching Model - Polytopic Model with SWF

— Polytopic Model with DWF — Filtered inputs

Figure 4.2: Comparison between the switching model and the polytopic model with the classical static weighting functions (SWF) and with the proposed dynamic weighting functions (DWF) for the case of a voltage-controlled boost converter. 

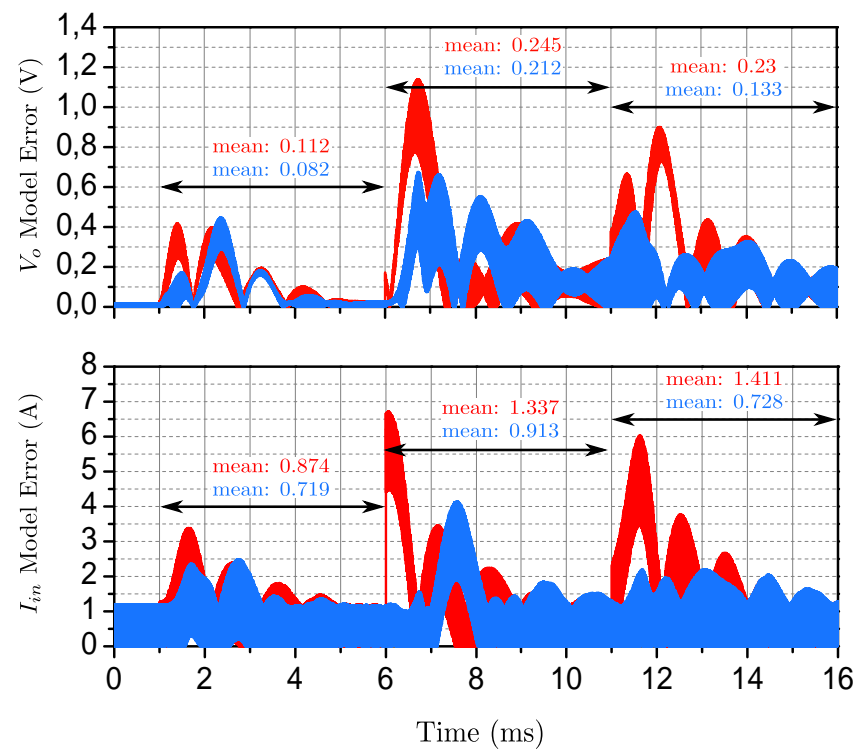

— Polytopic Model with SWF — Polytopic Model with DWF

Figure 4.3: Error signals between the switching model and the polytopic model with the classical static weighting functions (SWF) and with the proposed dynamic weighting functions (DWF) for the case of a voltage-controlled boost converter.
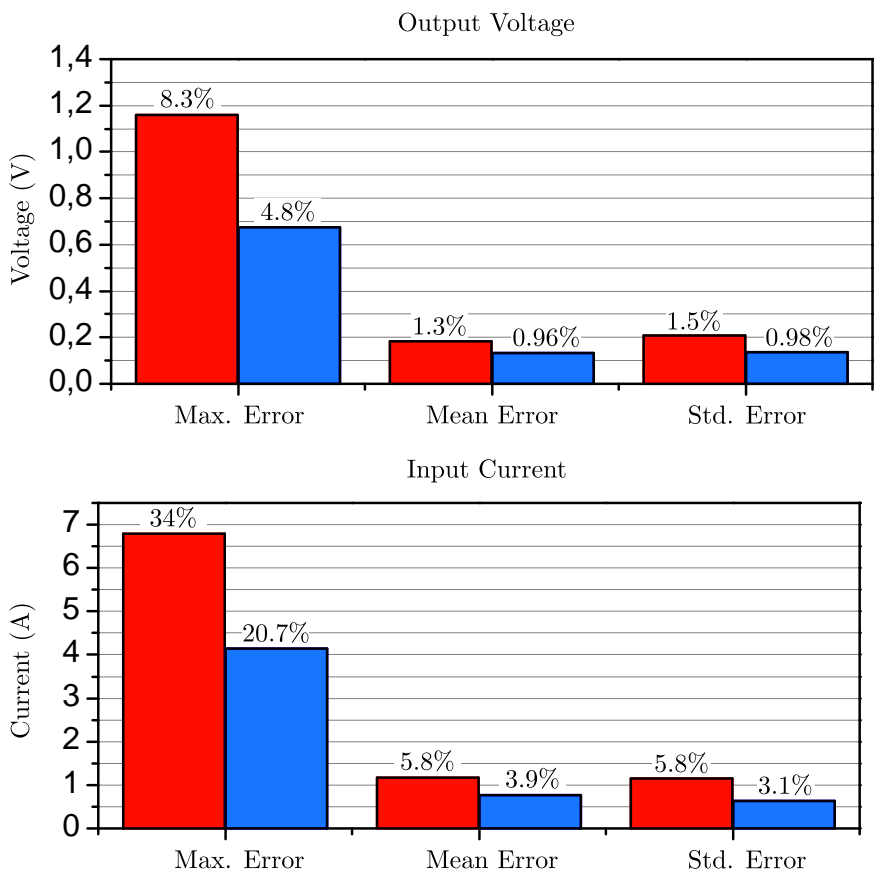

— Polytopic Model (SWF) — Polytopic Model (DWF)

Figure 4.4: Details about the performance of the polytopic model with the classical static weighting functions (SWF) and with the proposed dynamic weighting functions (DWF) for the case of a voltage-controlled boost converter. 


\subsection{Voltage-controlled buck converter in DCM and CCM case}

The case of the voltage-controlled buck converter with DCM is very challenging due to the strong variation of the dynamic behavior of the converter for different values of the output current (see Fig. 3.7). The modeling approaches that consider a linear dynamic behavior certainly will not be able to represent the dynamic behavior of the converter in DCM. The previous chapter showed that the polytopic model can represent the small-signal behavior of the converter around any operating point, but it provides a poor estimation of the dynamic behavior of the converter when a large step in the inputs occurs.

The same polytopic model that was detailed in Section 3.1.1 is used and the response of the model with static and dynamic weighting functions is compared. The transfer function that provides dynamic to the weighting function is designed using (4.4) and the poles of the small-signal model obtained around $I_{o}=3 \mathrm{~A}$. The resulting transfer function is:

$$
\tau(s)=\frac{438243.6}{s^{2}+1521.7 s+438243.6}
$$

This transfer functions is introduced between the output current (the only input variable in this case) and the input of the static weighting functions, as depicted in Fig. 4.1. In Fig. 4.5 the response of the polytopic model with static and dynamic weighting functions is compared with the switching model response to different load steps. Initially the load connected to the EPC demands an output current of $I_{o}=1 \mathrm{~A}$. At time $t=1 \mathrm{~ms}$ a new resistor is added in parallel to the load, increasing the output current to $I_{o}=2 \mathrm{~A}$. Because of the sharp step in the output current, the polytopic model with static weighting functions changes sharply to the small-signal model obtained around the final value of the output current, however the model using the dynamic weighting functions has a dynamic limitation in the rate of change among small-signal models related with the poles of the system, so the transition of the dynamic weighting functions is slower. The model with static weighting functions has a underestimation of the voltage drop of about $20 \%$, whereas the model with dynamic weighting functions follows the response of the converter very accurately during this step.

At time $t=31 \mathrm{~ms}$ another resistor is connected in parallel with the load increasing the output current to $I_{o}=4 \mathrm{~A}$. This load step leads the converter to change from DCM to CCM. As in the previous case, the static weighting functions change sharply from the initial to the final operating point, whereas the dynamic weighting function makes a transition from the initial point, $I_{o}=2 \mathrm{~A}$, to the intermediate point $I_{O}=3 \mathrm{~A}$, and ultimately to the final point $I_{o}=4 A$, which corresponds with the behavior of the converter in CCM. As the behavior of the converter is very different in DCM and CCM, and the converter does not change its dynamic behavior instantly, there is a substantial mismatch between the response of the converter and the model with static weighting functions. The model with dynamic weighting functions has an very good accuracy at the initial part of the transitory, but when the small-signal model corresponding to the CCM is activated there is a sharp transition that does not correspond to the real behavior of the converter.

At time $t=51 \mathrm{~ms}$ the resistor that was connected in the previous load step is disconnected, lowering the current demand down to $I_{o}=2 \mathrm{~A}$. First, it is interesting to notice the different dynamic behavior of the converter when it changes from DCM to CCM and when the transition is from CCM to DCM, as in this case. In this transition, none of the models are able to estimate the behavior of the converter. The model with static weighting functions uses directly the small-signal model corresponding to $I_{o}=2 \mathrm{~A}$, which overestimates the overshoot of the output voltage (with twice the voltage deviation). On the other hand, the model with dynamic weighting functions makes a transition among small-signal models that do not reflect the response of the converter. 

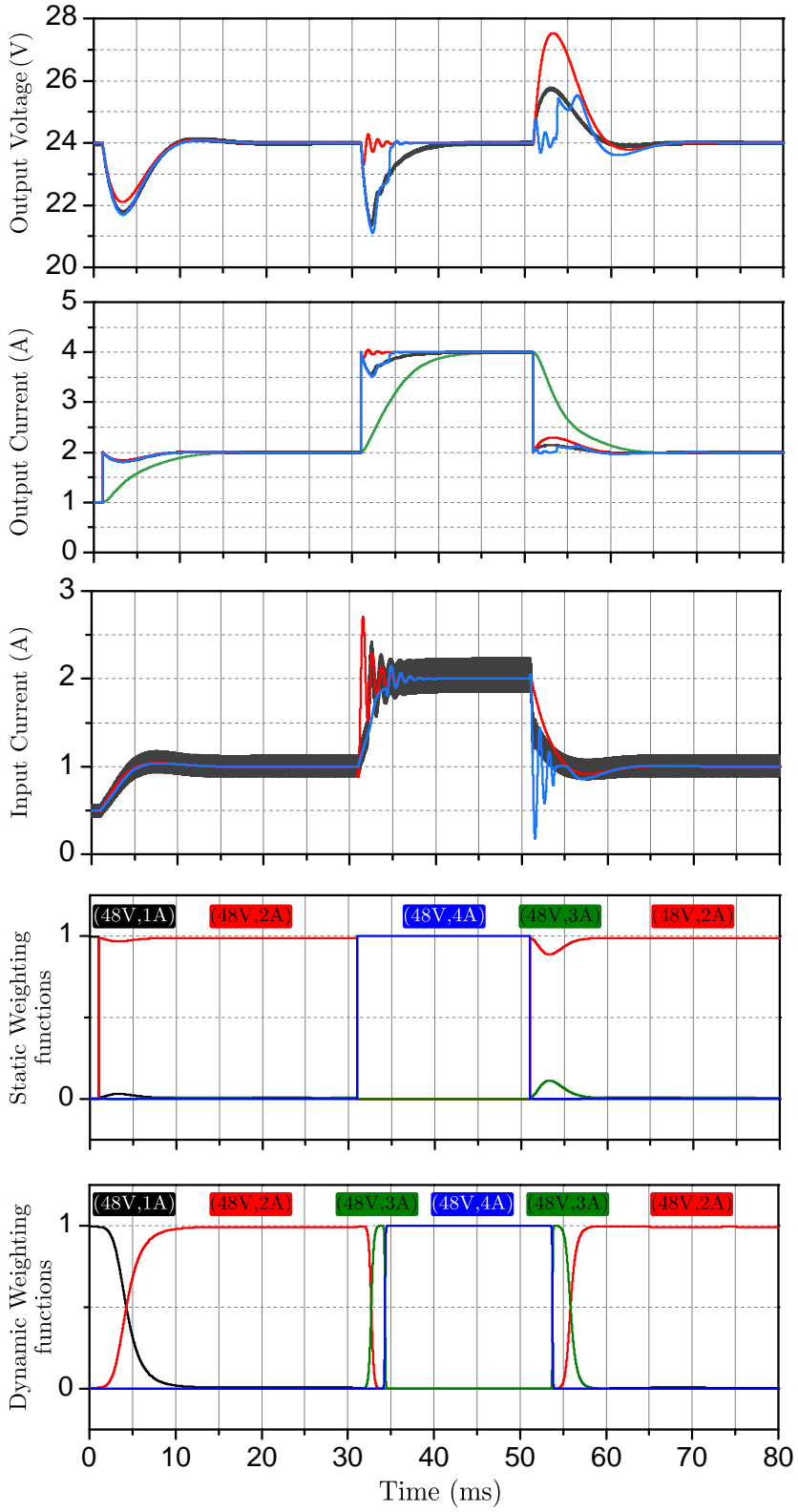

— Switching Model
— Polytopic Model with DWF — Filytopic Model with SWF
— Fits

Figure 4.5: Comparison between the switching model and the polytopic model with the classical static weighting functions (SWF) and with the proposed dynamic weighting functions (DWF) for the case of a voltage-controlled buck converter in DCM and CCM.

The error between the switching model and the two blackbox models obtained is shown in Fig. 4.6 and Fig.4.7. In this case, the proposed blackbox model improves the estimation of the behavior of the converter, however it is not able to reproduce accurately the transition from DCM to CCM and vice-versa. In the next chapter a new modeling approach will be proposed in order to account for strong nonlinearities in the dynamic behavior of the EPCs, such as the one presented in this case. 

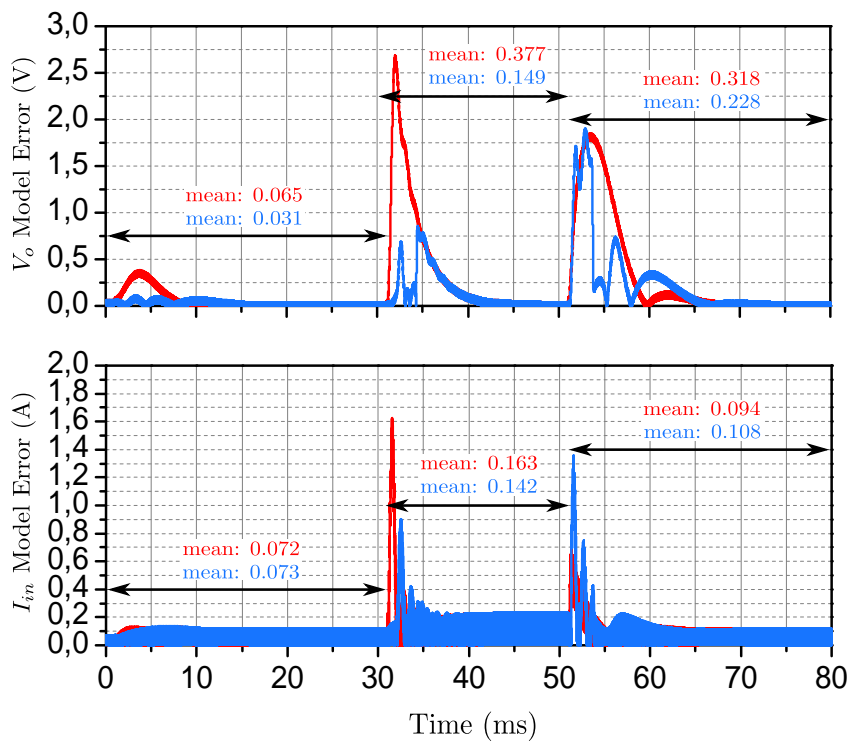

— Polytopic Model with SWF — Polytopic Model with DWF

Figure 4.6: Error signals between the switching model and the polytopic model with the classical static weighting functions (SWF) and with the proposed dynamic weighting functions (DWF) for the case of a voltage-controlled buck converter in DCM and CCM.

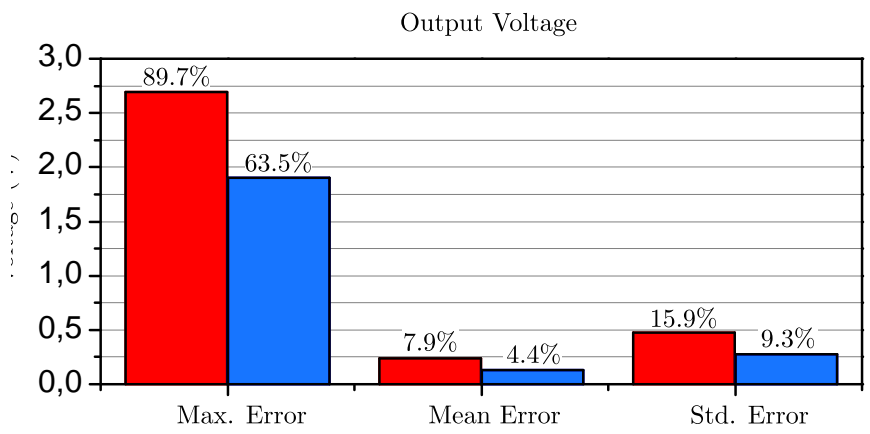

Input Current

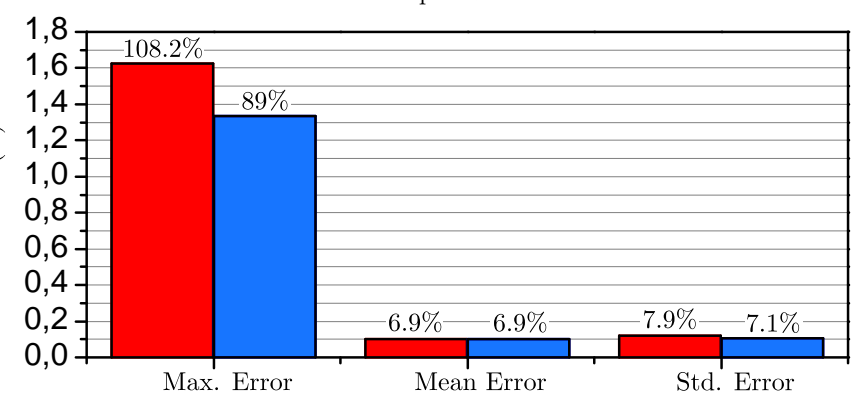

- Polytopic Model (SWF) _ Polytopic Model (DWF)

Figure 4.7: Details about the performance of the polytopic model with the classical static weighting functions (SWF) and with the proposed dynamic weighting functions (DWF) for the case of a voltage-controlled buck converter in DCM and CCM. 


\subsection{Voltage-controlled synchronous buck converter with double-loop control and current saturation case}

The previous chapter showed that the G-parameters model was not able to account for changes in the operation mode of the converters, as the case of a voltage-controlled synchronous buck converter with double-loop control and current limitation. The polytopic model was able to approximate the behavior of the converter in both operation modes, but it is not able to provide a good estimation of the dynamic behavior during the transition from voltage to current mode or vice-versa. A modification to the reference of the Wiener-Hammerstein model was proposed in order to capture the dynamic behavior of the converter in all conditions, providing an accurate estimation. In Section 3.2 it was shown that for changes in the operation mode of converters it is more convenient to establish the conditions to change from one mode to another sharply, instead of using double sigmoids as weighting functions. However, in this section the variables of the model will be filtered with the characteristic filter shown in (4.4).

The goal of this approach is to approximate the dynamic behavior of the state variables using the input-output variables available and the filter mentioned before. The performance of the polytopic model using directly the input-output variables and the approximated state variables will be compared in this section for the example shown in the previous chapter. The use of the input variables filtered with the characteristic filter and the conditions to change from voltage to current mode is what it will be named dynamic weighting function in this case, whereas the use of input-output variables will be named static weighting functions.

The characteristic filter is obtained using the poles of the transfer functions that were identified when the converter is working in voltage mode. Using the transfer functions identified, shown in Table 3.5, and the definition of characteristic filter shown in (4.4), the corresponding transfer functions is:

$$
\tau(s)=\frac{2.52 e 8}{s^{2}+5084.7 s+2.52 e 8}
$$

For the sake of clarity, only the output impedance will be considered in this case. Three options will be compared: the polytopic model using the input-output variables and constant initial conditions; the polytopic model using the DWF and constant initial conditions; and the polytopic model using the DWF and a initial condition for the voltage of the model obtained in current mode that depends on the output voltage value of the model in voltage mode in the instant in which the change occurs. A scheme of the three models is depicted in Fig 4.8, where the superscript $v$ correspond to the elements related with the converter in voltage mode, and the superscript $i$ corresponds to the elements related with the converter in current mode.

The conditions considered for the change between operation modes are the same that the ones detailed in Section 3.2. The model with static weighting functions uses directly the output current measured in the load for the comparison with the limit current. The model with dynamic weighting functions includes the characteristic filter and uses the filtered output current for the comparison with the limit current. Finally, the model with variable initial conditions uses a sample\&hold block that sets the value of the model in voltage mode, in the instant when the conditions for the change are met, as nominal output voltage of the model in current mode. Notice that this strategy can be applied to the current mode because the converter does not regulate the output voltage, in case it is applied to the converter in current mode, the operating point must be the current reference. Finally, all the models keep the output current of the current mode model in the limit value $I_{o}=6 \mathrm{~A}$ when it is not activated, otherwise the initial value of the output voltage of this model in 
the instant of change between modes would not be the nominal voltage, which results in mismatches during the transition.

The comparison between the three models and the switching model is presented in Fig. 4.9. Initially there is a resistor connected in the load demanding $I_{O}=1 \mathrm{~A}$. At time $t=1 \mathrm{~ms}$ a resistor is connected in parallel with the load increasing the output current to $I_{o}=3 \mathrm{~A}$. As the converter has not reached the current limit $\left(I_{o}=6 \mathrm{~A}\right)$, all the models work in voltage mode and their performance is the same.

At time $t=2 \mathrm{~ms}$ another resistor is connected to the load increasing the load demand above $I_{o}=6 \mathrm{~A}$. Since the current demanded is above the current limit, the converter enters in current mode during this load step. However, as shown in the previous chapter, the transition is not instantaneous. The polytopic model with static weighting functions changes sharply to current mode and its dynamic behavior is substantially different from the response of the converter. The model with dynamic weighting functions and constant initial conditions for the output voltage of the model in current mode has an initial behavior equal to the model (it keeps working in voltage mode), but after the transition to current mode (approximately $t=2.15 \mathrm{~ms}$ ) the model jumps to $V_{o}=12 \mathrm{~V}$ and the accuracy of the estimation is lost from that point on. Finally, the model with dynamic weighting functions and a variable initial condition for the nominal voltage of the model in current mode is able to track the dynamic response of the converter with high accuracy during the transient response.

At time $t=3 \mathrm{~ms}$ a resistor is added to the load, but as the converter is in current mode it causes a voltage drop, which is well reflected by the three models. Finally, at time $t=4 \mathrm{~ms}$ the last two resistors are disconnected, leading the converter back to voltage mode. Again, the transition is not instantaneous. In this case, the output voltage has to be restored before changing from current to voltage mode, which is taken into account in the conditions to switch between operation modes. After the output voltage has reached the nominal value, $V_{o}=12 \mathrm{~V}$, all the models change to voltage mode, but due to the different initial conditions of the model working in voltage mode, there is a discontinuity in the response and none of the models is able to accurately track the response of the converter. As it was mentioned before, the operating point of the models in voltage mode cannot be modified because the converter is regulating this value, that is, the output impedance has a steady state value of zero, so if the operating point is changed the voltage reference will change, which is not acceptable.

The error between the switching model and the three blackbox models taken into account is included in Fig. 4.9, where it can be seen that the only differences are in the transitions between operation modes. It can be clearly noticed that the model proposed is able to account for the transition from voltage mode to current mode, where the error is practically at the same level as the switching ripple. However, none of the models considered is able to reproduce the transition from current mode to voltage mode. In the next chapter this case will be analyzed in more detail and it will be used to test the new modeling approach proposed therein.

Finally, in Fig. 4.10 the different measures of the overall error of the models are shown. In this case, the overall error analysis is not very illustrative because the difference among the models is restricted to only one of the steps. Besides, the maximum error of the models is in the transition from current mode to voltage mode, in which the three approaches have the same behavior. Nevertheless, it has been included for the sake of completeness. 
Models

SWF with constant IC
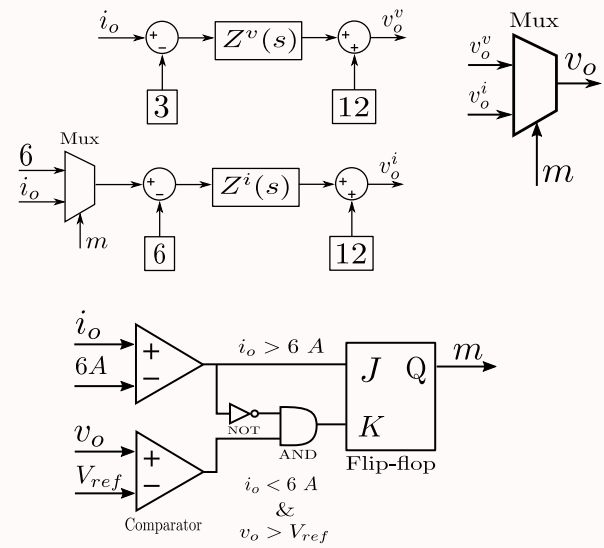

DWF with constant IC

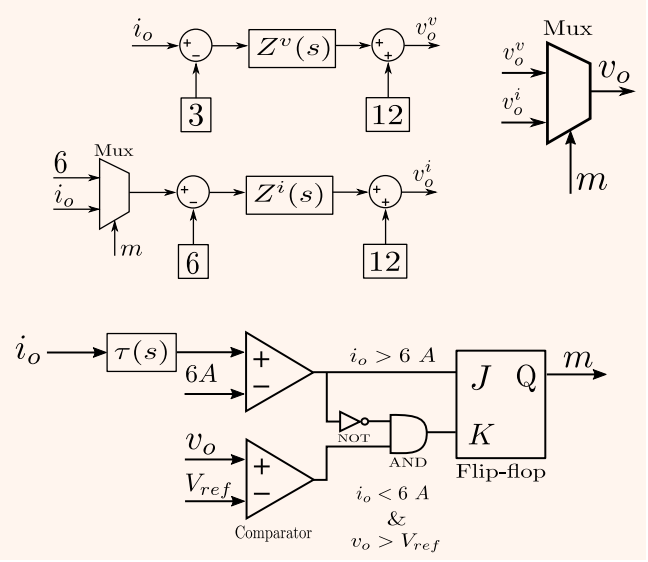

DWF with variable IC

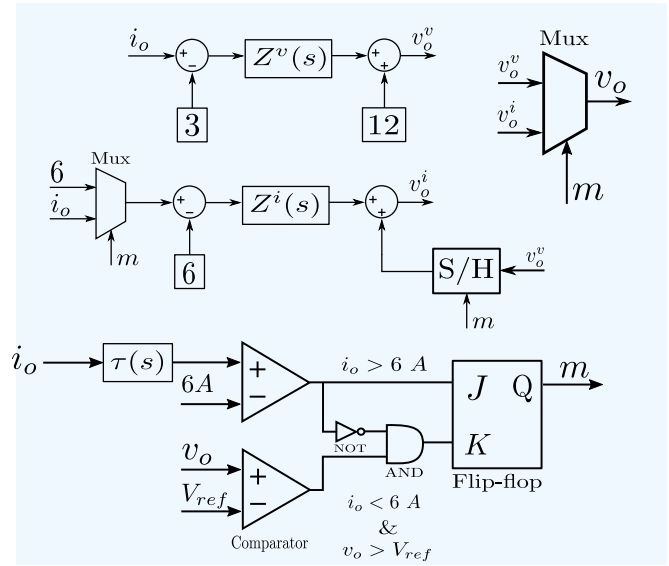

Figure 4.8: Scheme of the polytopic models with input-output variables and constant initial conditions (IC), DWF and constant initial conditions, and DWF and variable initial conditions.

\section{Load}

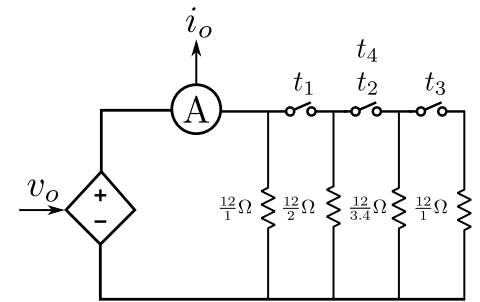



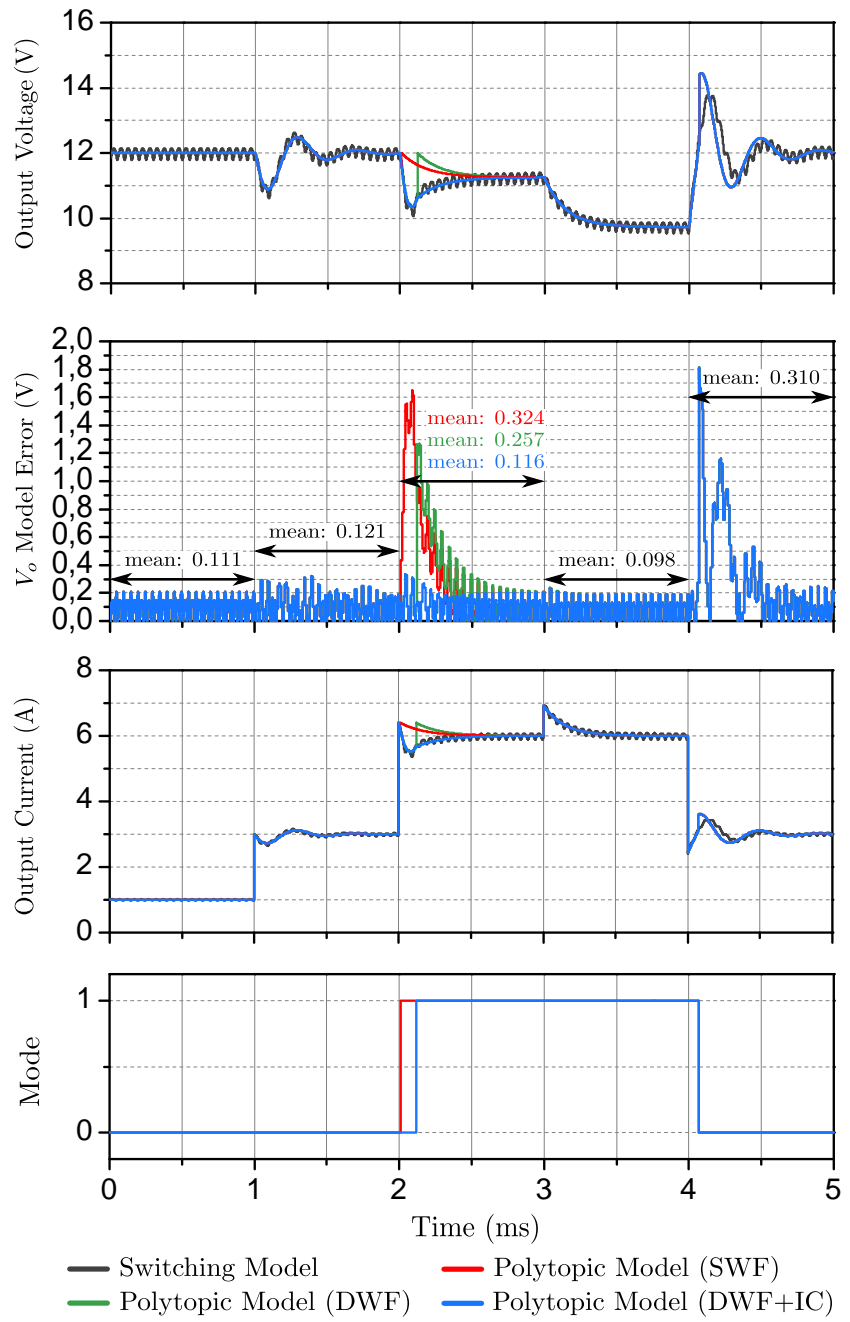

Figure 4.9: Comparison between the switching model and the polytopic model with the classical static weighting functions (SWF), with the dynamic weighting functions (DWF), and with the dynamic weighting functions plus a variable initial condition of the output voltage for the model in current mode for the case of a voltage-controlled synchronous buck converter with a double-loop control.

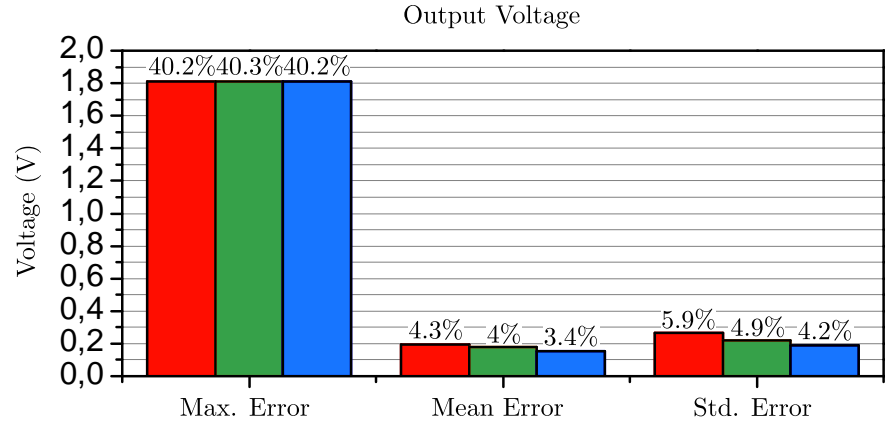

- Polytopic Model (SWF) - Polytopic Model (DWF)

- Polytopic Model (DWF+IC)

Figure 4.10: Details about the performance of the polytopic model with the classical static weighting functions $(\mathrm{SWF})$, with the dynamic weighting functions (DWF), and with the dynamic weighting functions plus a variable initial condition of the output voltage for the model in current mode for the case of a voltage-controlled synchronous buck converter with a double-loop control. 


\subsection{Conclusions}

In this chapter the concept of dynamic weighting function has been introduced. This method aims to approximate the dynamic of the state variables by filtering the inputoutput variables of the model with a transfer function designed from the information of the poles of the system. This transfer function has a unitary gain to avoid the distortion of the variables in the steady state. The rationale of this proposal is that the nonlinear behavior in EPCs can be due to nonlinearities related with the state variables, which are not available in blackbox models. By using what it has been named as the characteristic filter, the input to the traditional static weighting functions is not directly the input-output variable, which can change sharply, but these variables filtered by the mentioned characteristic filter. This causes that the transition among the small-signal models which integrated the polytopic model is necessarily as sharp as the step in the input variables, but it is limited by the dynamic of the poles of the system, which is related with the dynamic of the state variables.

It has been demonstrated with different examples that the dynamic behavior of operating point dependent EPCs can be approximated with better accuracy when they are subjected to large and sharp steps in the input variables. The polytopic model with dynamic weighting functions makes a transition through all the small-signal models between the initial operating point and the final operating point, whereas the polytopic model with static weighting functions change abruptly and directly to the final operating point when the step in the input variables is sharp. This limitation in the rate of change among smallsignal models, that is, in the rate of change in the dynamic behavior of the EPCs, has been proved to be a better approximation of the real behavior of switching converters.

Furthermore, the dynamic of EPCs with discontinuous and continuous conduction modes is improved using this method. However, when the converter has a very different dynamic behavior depending on the operating point, as it is the case in converters in DCM and CCM, the transition from one to another is still not accurate.

Similarly, the estimation of the dynamic behavior of converters with different operation modes, as the case of a synchronous buck converter with a double-loop control and current limitation, is improved by using the dynamic weighting functions. In this case, the dynamic of inductor current is well captured by using the characteristic filter, therefore the delay between the load step that reaches the current limit and the actual moment when the converter enters in current mode is well modeled. Notice that during this transition the converter starts behaving with the dynamic of the voltage mode and during the transient time it changes to current mode. By giving the initial value of the output voltage to the model in current mode in the instant when the transition occurs, the response of the converter is represented neatly. However, the transition from current mode to voltage mode is not well reflected by this model, as the initial condition of the output voltage when the mode changes cannot be given to the model in voltage mode, as it was discussed above.

The proposal of the concept of dynamic weighting functions to improve the transition among local models in polytopic models is an important contribution of this thesis.

In the next chapter the cases where the polytopic model with dynamic weighting functions is not able to represent accurately the dynamic response of the EPC will be analyzed. 


\section{Parameter varying transfer functions with dynamic weighting functions}

\footnotetext{
$C$

hapter 3 showed that the polytopic model with static weighting functions is able to represent accurately the response of EPCs with variable dynamic behavior around any operating point as long as the perturbations in the input variables are small. In Chapter 4 the dynamic weighting functions were proposed to improve the performance of the polytopic model for large and sharp steps in the input variables of the model, which effectively increased the accuracy of the polytopic model during the transient response. However, in case of strong dynamic variations in the response of the converter, as it was the case of the buck converter with discontinuous and continuous conductions modes and the synchronous buck converter with a double-loop control and current saturation, the model was not able to represent correctly all the transition from one mode to the other.

The reason why the polytopic model with dynamic weighting functions is not able to represent accurately the behavior of the converter with strong dynamic changes is that this model consists of different small-signal models which are simulated in parallel and the overall model is a weighted combination of these responses or, in case of a sharp transition, the model switches between the response of one small-signal model to the other. When the converter has very different dynamic responses for different operating points, the initial conditions during the transient response become very important. For instance, if the dynamic of the model in the initial operating point is much slower than the dynamic at the final operating point, the latter model will have already reached the steady state in the moment in which its weighting functions are activated, whereas the previous models will be in the middle of the transitory leading to a discontinuity in the response of the model that do not correspond with the actual behavior of the converter (see the transition from DCM to CCM in Fig. 4.5 and the transition from current to voltage mode in Fig. 4.9). Similarly, if the dynamic of the model in the initial operating point is much faster than the dynamic of the model in the final operating point, the model will reach the steady state before the weighting function of the next model is activated, which will be at some point of the transitory, leading to a very poor approximation (see the transition from CCM to DCM in Fig. 4.5).
} 
In this chapter a different approach to modify the dynamic of the model is proposed in order to be able to represent EPCs with very different dynamic behaviors and the transient response from one mode of operation to the other.

\subsection{Parameter varying transfer functions}

In Section 2.2.2 it was shown that the polytopic model is a kind of LPV model. These models are characterized by having time-varying parameters that account for the variation in the dynamic response of the converters. This method is commonly applied to the parameters of the state space matrices [93]. One advantage of using one model with variable parameters, instead of a weighted combination of local models, is that the problem of initial conditions is avoided, i.e. when a parameter is varied it modifies the dynamic behavior of the model from the point in which the system was in the previous instant.

Using a blackbox approach, it is more convenient to use input-output models due to the lack of details about the internal architecture of the EPCs. This is the reason why the blackbox polytopic model is represented with transfer functions instead of in the state space. Therefore, the goal of this section is to use a parameter varying approach with transfer functions, where the variation of the parameters is performed using the dynamic weighting functions introduced in the previous chapter. Combining these two strategies it is expected to avoid the errors due to different initial conditions of the local models and to keep the capability of the dynamic weighting functions of approximating the rate of change of the parameters to the dynamic of the state variables.

First, a practical method to represent Parameter Varying Transfer Functions (PVTF) is detailed. In order to follow this approach it is more convenient to use discrete time systems. The goal is to express the output variable as a combination of a set of parameters that multiply the current value of the input and the past values of the input and output variables. Consequently, the general second order transfer function:

$$
\frac{y(z)}{u(z)}=G(z)=\frac{a_{1} z^{2}+a_{2} z+a_{3}}{z^{2}+b_{1} z+b_{2}}
$$

can be expressed as:

$$
y(z)\left(z^{2}+b_{1} z+b_{2}\right)=u(z)\left(a_{1} z^{2}+a_{2} z+a_{3}\right)
$$

which can be represented as:

$$
y(z)\left(1+b_{1} z^{-1}+b_{2} z^{-2}\right)=u(z)\left(a_{1}+a_{2} z^{-1}+a_{3} z^{-2}\right)
$$

so the expression of the output variable can be represented as:

$$
y(z)=u(z)\left(a_{1}+a_{2} z^{-1}+a_{3} z^{-2}\right)-y(z)\left(b_{1} z^{-1}+b_{2} z^{-2}\right)
$$

rearranging terms it can be expressed as:

$$
y(z)=a_{1} u(z)+a_{2} u(z) z^{-1}+a_{3} u(z) z^{-2}-b_{1} y(z) z^{-1}-b_{2} y(z) z^{-2}
$$

or equivalently: 


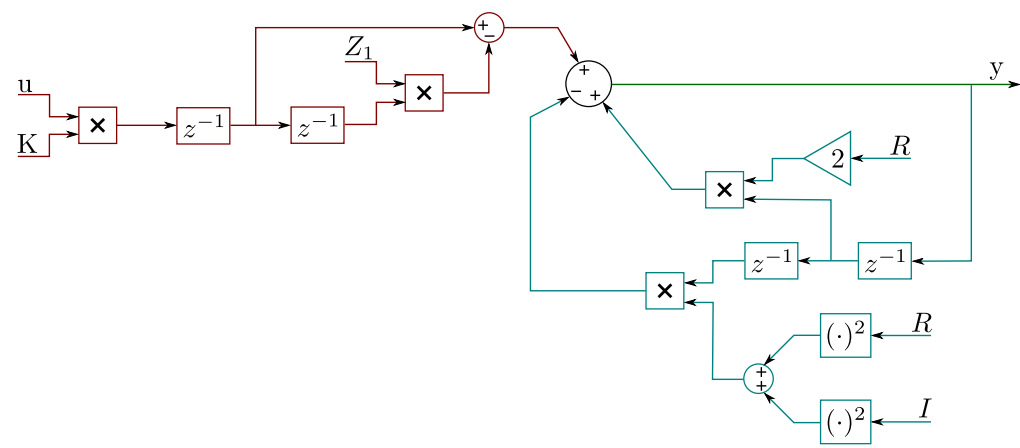

Figure 5.1: Block diagram of a parameter varying transfer function with one zero and a pair of complexconjugate poles.

$$
y(n)=a_{1} u(n)+a_{2} u(n-1)+a_{3} u(n-2)-b_{1} y(n-1)-b_{2} y(n-2)
$$

This expression is very convenient to represent parameter varying transfer functions, as it is a combination of parameters that multiply present and past values of the input and output signals. These parameters can be easily changed in each time instant, affecting the next value of the output variable and considering the past values of the input and output signals. In order to work with meaningful information about the system, the parameters considered can be expressed as a function of the gain, zeros, and poles of the transfer function. For instance, the transfer function:

$$
\frac{y(z)}{u(z)}=G(z)=\frac{K\left(z-Z_{1}\right)}{(z-R+j I)((z-R-j I))}
$$

corresponding to a system with gain $K$, one zero, $Z_{1}$, and a pair of complex-conjugate poles with real part $R$ and imaginary part $I$, can be expressed as:

$$
y(z)=K u(z) z^{-1}-Z_{1} K u(z) z^{-2}+2 R y(z) z^{-1}-\left(R^{2}+I^{2}\right) y(z) z^{-2}
$$

or equivalently:

$$
y(n)=K u(n-1)-Z_{1} K u(n-2)+2 R y(n-1)-\left(R^{2}+I^{2}\right) y(n-2)
$$

These expressions can be represented in a block diagram that can be implemented in most of the commercial simulators. The block diagram of the example (5.7) is depicted in Fig. 5.1. In case the small-signal models have a different number of poles and zeros, the most complex structure should be implemented. This scheme shows the output of one transfer function. The G-parameters model will consists of four parameter varying transfer functions that can be implemented in the same manner.

In the polytopic model, the outputs of each independent local model were introduced to the weighting functions. In this model the value of the parameters are the ones weighted according to the operating point in which the system is working. Similarly, dynamic weighting functions can be used to control the rate of change of the parameters.

Finally, the output of the model can control a controllable source connected to the load and the output current can be feedback to the model. Some examples of the construction of this model will be shown in the following for the cases where none of the previous modeling approaches were able to represent the dynamic of the EPC in all the possible scenarios. 


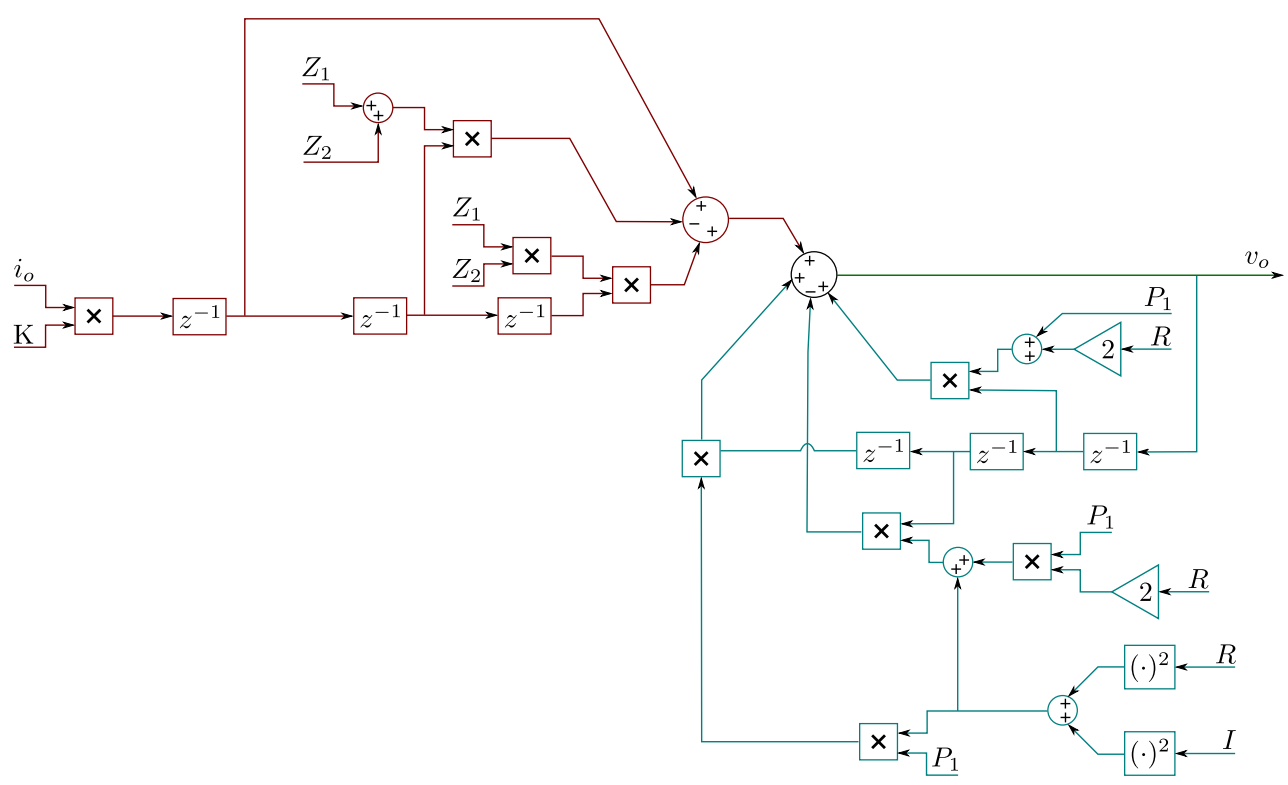

Figure 5.2: Block diagram of the parameter varying transfer function of the output impedance for the voltage-controlled buck converter in DCM and CCM.

\subsection{Voltage-controlled buck converter in DCM and CCM case}

The previous chapters showed that none of the modeling structures available, nor the proposed polytopic model with dynamic weighting functions, were able to represent the dynamic of the voltage-controlled buck converter in DCM and CCM. The main reason is the strong variability of its dynamic behavior that depends on the value of the output current. The polytopic model with dynamic weighting functions was able to improve the estimation of the dynamic response of the converter for some of the transitions, but in the others the errors were considerable due to the large differences in the settling time of the different small-signal models and the corresponding mismatch of initial conditions in the moment in which the transition between local models occurs. In this section the performance of the proposed parameter varying transfer functions with DWF will be compared with the response of the switching model and the polytopic model with DWF.

In Table 3.2 the four local models identified for different values of the output current of this converter were presented. For the sake of clarity only the output impedance will be studied, as it is considered the most relevant transfer function in this example. However, in the simulation both output variables will be shown. It can be seen that the transfer functions of the output impedance has one zero and a pair of complex-conjugate poles when the converter is in DCM, and two zeros and one real pole plus two complex-conjugate poles when the converter is in CCM. Therefore, a structure that includes the latter case will be implemented as it is the most general case, which is depicted in Fig. 5.2.

The transfer functions in $\mathrm{z}$ domain are represented in Table 5.1. From this representation it is straightforward to extract the parameters that will be used in the parameter varying transfer functions. The block diagram of the weighted combination of these parameters is represented in Fig. 5.3.

The variation in the dynamic response of the converter will be considered also in the design of the dynamic weighting function. Instead of a traditional transfer function with the poles of one of the local models, a parameter varying transfer function will be used. The parameters of the characteristic filter will also change with the dynamic weighting functions. In order to simplify the model, a first order transfer function will be used as 
Table 5.1: Parameters of the identified transfer functions of the voltage-controlled buck converter for different values of the output current.

\begin{tabular}{|c|c|}
\hline Output Current & Output impedance, $Z(z)$ \\
\hline \hline$I_{o}=1 \mathrm{~A}$ & $\frac{19.836 e-4(z-1)}{(z-0.99985+3.852 e-4 i)(z-0.99985-3.852 e-4 i)}$ \\
\hline$I_{O}=2 \mathrm{~A}$ & $\frac{19.819 e-4(z-1)}{(z-0.99974+4.185 e-4 i)(z-0.99974-4.185 e-4 i)}$ \\
\hline$I_{o}=3 \mathrm{~A}$ & $\frac{22.476 e-4(z-1)(z-0.9991)}{(z-0.9978)(z-0.99962+2.914 e-4 i)(z-0.99962-2.914 e-4 i)}$ \\
\hline$I_{o}=4 \mathrm{~A}$ & $\frac{20.869 e-4(z-1)(z-0.9978)}{(z-0.9992)(z-0.99936+57.04 e-4 i)(z-0.0 .99936-57.04 e-4 i)}$ \\
\hline
\end{tabular}

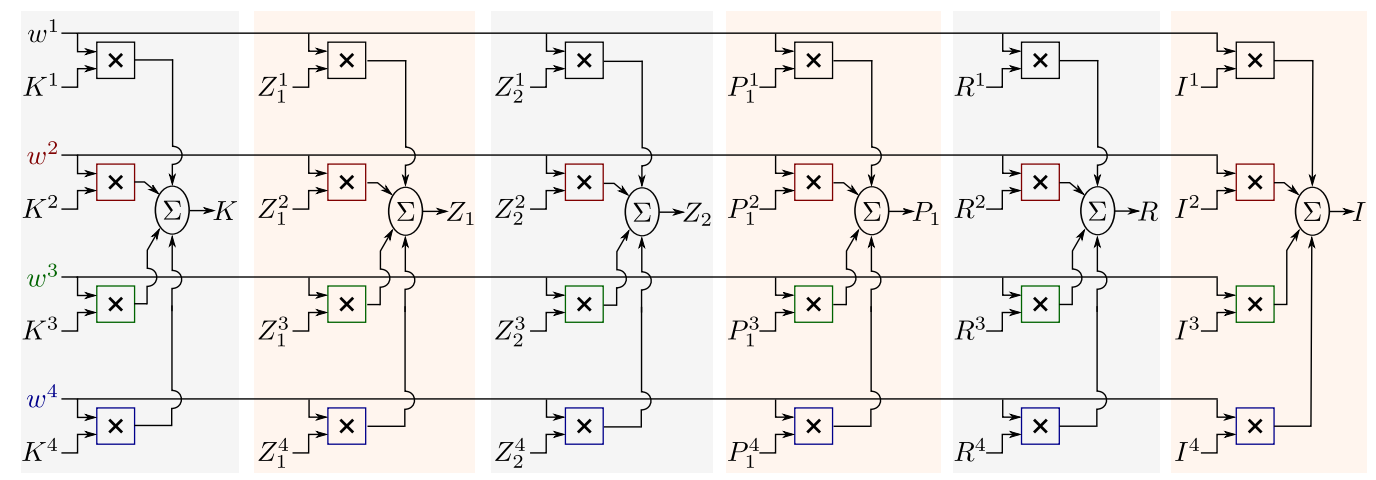

Figure 5.3: Block diagram of the weighted combination of each parameter for the output impedance parameter varying transfer function.

characteristic filter, where the poles correspond to the natural frequency of the complexconjugate poles of the different local models. The four corresponding transfer functions are represented in Table 5.2. Those parameters will be used in the parameter varying transfer function that will be included in the dynamic weighting function. The block diagram of the dynamic weighting functions designed with a parameter varying transfer function is depicted in Fig. 5.4, where $w_{s}$ corresponds to the static weighting function presented in Table 5.3. A block diagram of the model is depicted in Fig. 5.5.

The performance of the model is compared with the switching model and the polytopic model with DWF in Fig. 5.6. The converter is initially delivering an output current of $I_{o}=1 \mathrm{~A}$. At time $t=1 \mathrm{~ms}$ a resistor is connected in parallel with the load increasing the output current demand to $I_{O}=2 A$. It can be seen that both models reproduce the behavior of the converter within the DCM with high accuracy, however the suitable weighting functions for the polytopic model are slower than the ones that works better with the parameter varying transfer function. This is explained because the change in the parameter is faster than its effect of the output response of the transfer functions. At time $t=31 \mathrm{~ms}$ another resistor is connected in parallel with the load increasing the load demand to $I_{o}=4 \mathrm{~A}$, which leads to a transition from DCM to CCM. It can be seen how the 
Table 5.2: Parameters of the identified transfer functions of the voltage-controlled buck converter for different values of the output current.

\begin{tabular}{|c||c||c|}
\hline Output Current & Characteristic filter, $\tau(s)$ & Characteristic filter, $\tau(z)$ \\
\hline \hline$I_{o}=1 A$ & $\frac{437.3}{s+437.3}$ & $\frac{43.72 e-5}{(z-0.9996)}$ \\
\hline$I_{o}=2 A$ & $\frac{758}{s+758}$ & $\frac{75.77 e-5}{(z-0.9992)}$ \\
\hline$I_{O}=3 A$ & $\frac{1133}{s+1133}$ & $\frac{11 e-4}{(z-0.9989)}$ \\
\hline$I_{O}=4 A$ & $\frac{2503}{s+2503}$ & $\frac{25 e-4}{(z-0.9975)}$ \\
\hline
\end{tabular}
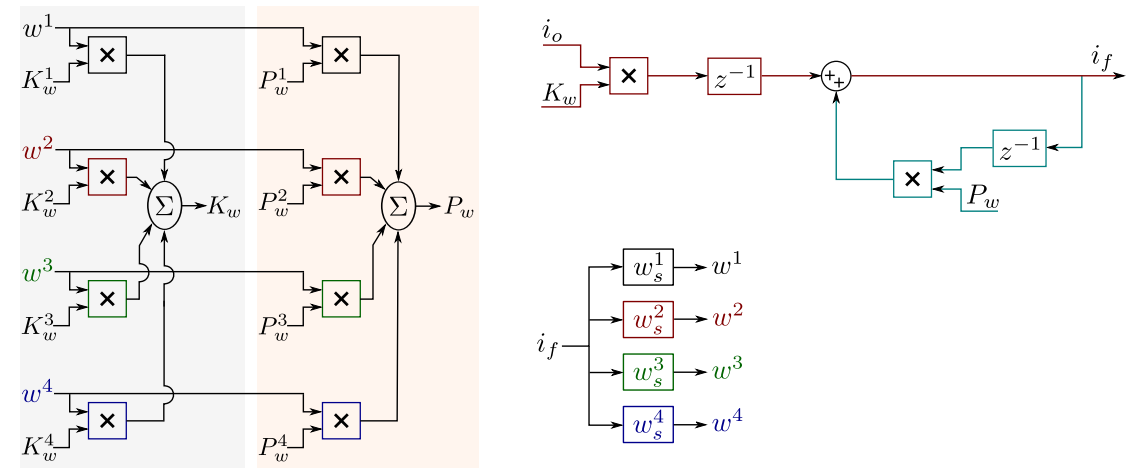

Figure 5.4: Block diagram of the dynamic weighting functions using a parameter varying transfer function.

Table 5.3: Parameters of the static weighting functions (SWF) implemented for the parameter varying transfer function model of the voltage-controlled buck converter in DCM and CCM.

\begin{tabular}{|c||c||c|}
\hline SWF & Slopes & Centers \\
\hline \hline$w_{s}^{1}$ & $m_{1}=30, m_{2}=30$ & $c_{1}=-\infty, c_{2}=1.3$ \\
\hline$w_{s}^{2}$ & $m_{1}=30, m_{2}=50$ & $c_{1}=1.3, c_{2}=2.6$ \\
\hline$w_{s}^{3}$ & $m_{1}=50, m_{2}=150$ & $c_{1}=2.6, c_{2}=3$ \\
\hline$w_{s}^{4}$ & $m_{1}=150, m_{2}=150$ & $c_{1}=3, c_{2}=\infty$ \\
\hline
\end{tabular}




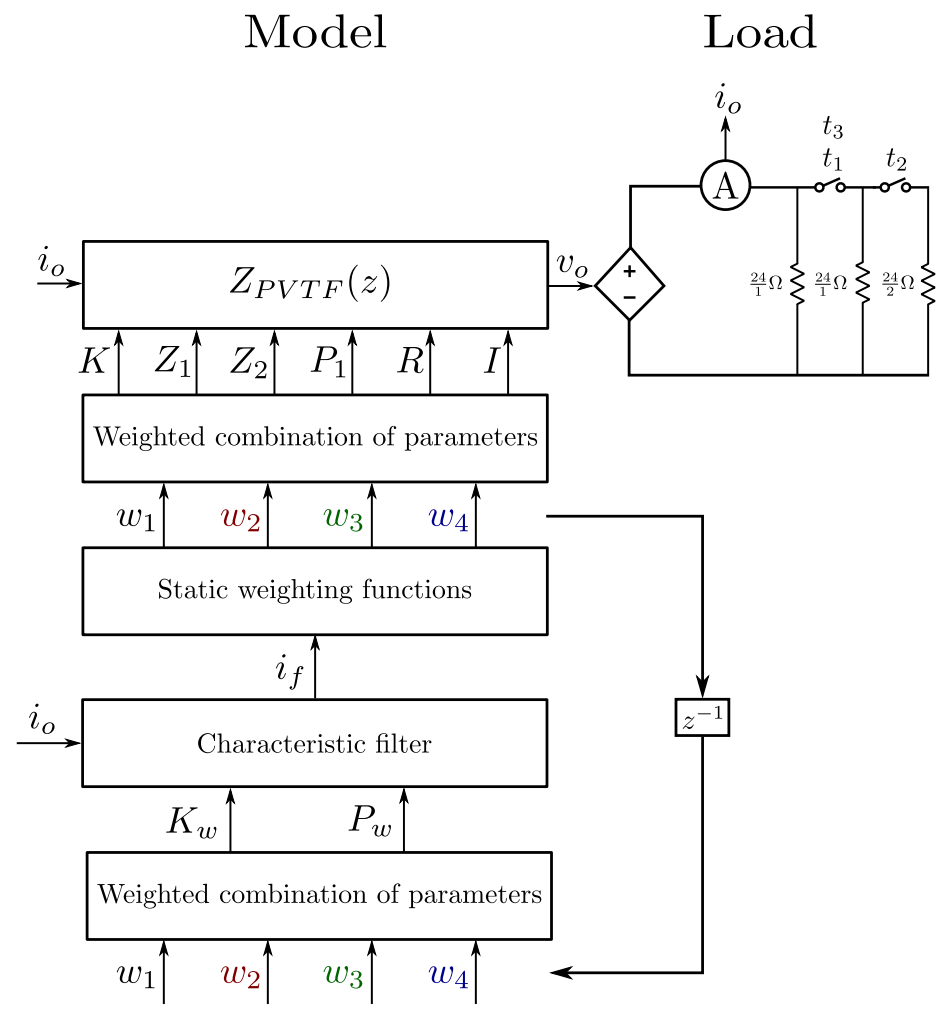

Figure 5.5: Block diagram of the parameter varying transfer functions model with DWF for the case of a voltage-controlled buck converter in DCM and CCM.

accuracy of the transition is substantially improved using the new approach. Besides, in the parameter varying transfer function model the discontinuity due to the activation of the model in CCM is avoided, resulting in a more appropriate result. Finally, at time $t=51 \mathrm{~ms}$ the last resistor is disconnected and a transition from CCM to DCM occurs. In this case the parameter varying transfer function model provides a highly accurate estimation, whereas the polytopic model with dynamic weighting functions has a very poor performance. This example shows that, overall, the parameter varying transfer function model provides a very good estimation of the response of a converter with strong dynamic variations. As it was justified before, this model avoids the effect of different initial conditions during the transition among small-signal models that affects the polytopic models. This approach changes the parameters of the transfer functions, affecting its dynamic but considering the previous values of the input and output signals.

In Fig. 5.7 the error between the switching model and the two blackbox modeling approaches considered is presented. The reduction of the error in both output variables is evident, with a error reduction of $52 \%$ in the transition from DCM to CCM and of $83 \%$ in the transition from CCM to DCM.

Finally, in Fig. 5.8 the different measures of the overall error of the models is shown. The reduction of the error is between $60 \%$ and $80 \%$ in the output voltage and between $12 \%$ and $70 \%$ in the input current. 

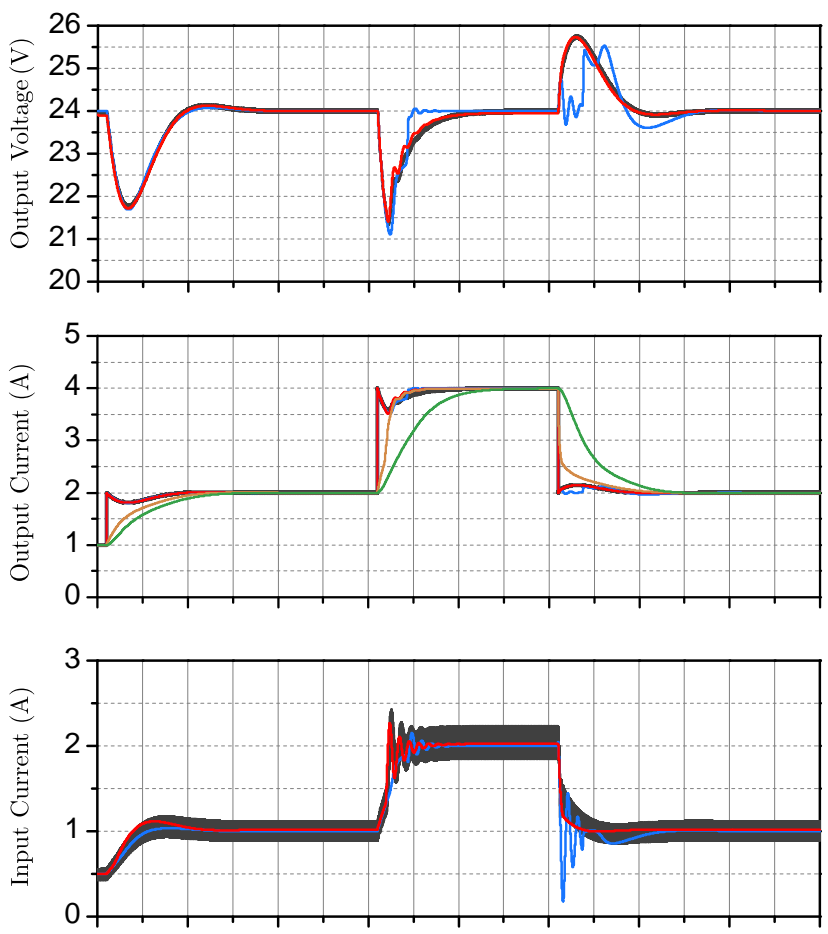

Polytopic Model with DWF

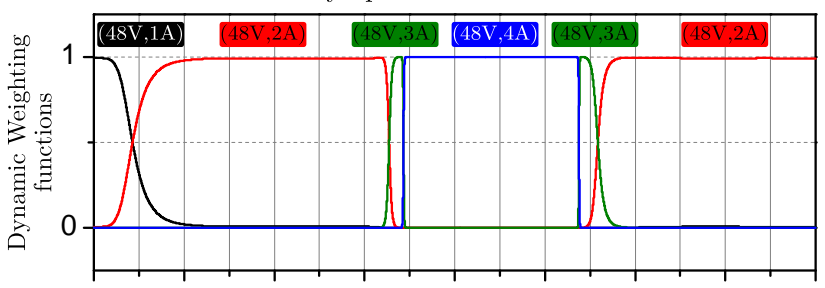

Variable Transfer Function Model with DWF

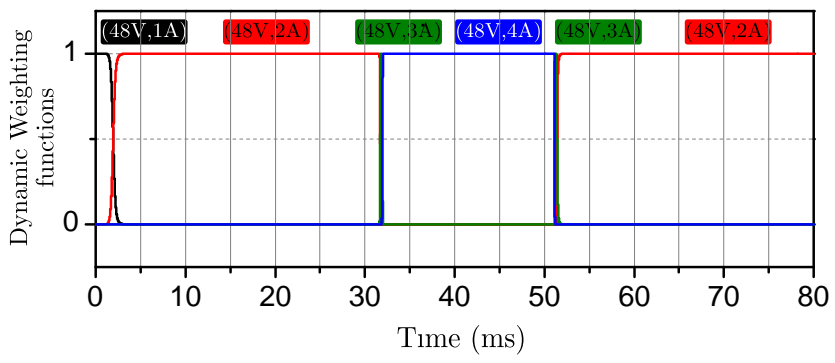

- Switching Model

- Polytopic Model with DWF

- Parameter Varying Transfer Function Model with DWF

— Filtered inputs (DWF) — Filtered inputs (PVTF)

Figure 5.6: Comparison between the switching model and the polytopic model with dynamic weighting functions (DWF) and the parameter varying transfer functions model with DWF for the case of a voltagecontrolled buck converter in DCM and CCM. 

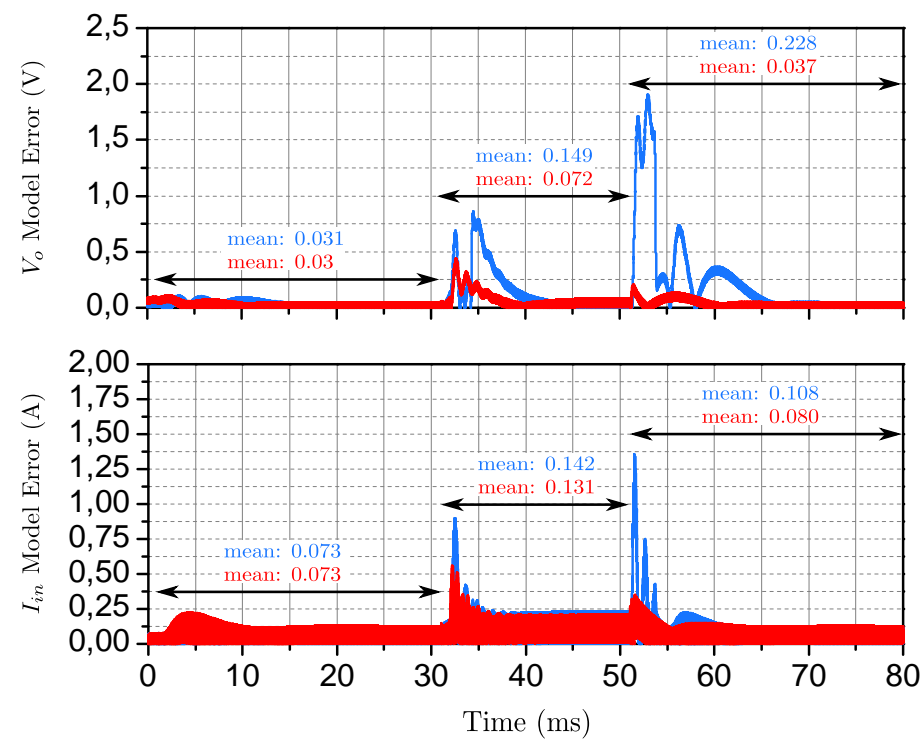

- Polytopic Model with DWF

- Parameter Varying Transfer Function Model with DWF

Figure 5.7: Error between the switching model and the polytopic model with dynamic weighting functions (DWF) and the parameter varying transfer functions model with DWF for the case of a voltage-controlled buck converter in DCM and CCM.
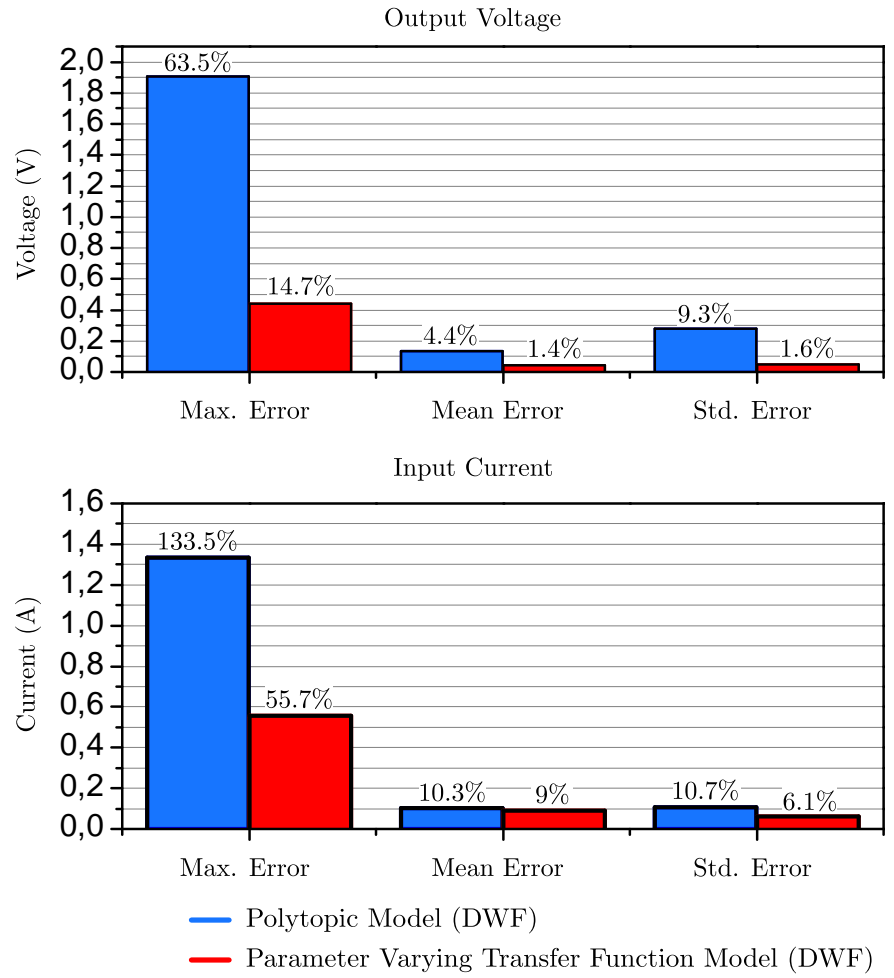

Figure 5.8: Details about the performance of the polytopic model with dynamic weighting functions (DWF) and the parameter varying transfer functions model with DWF for the case of a voltage-controlled buck converter in DCM and CCM. 


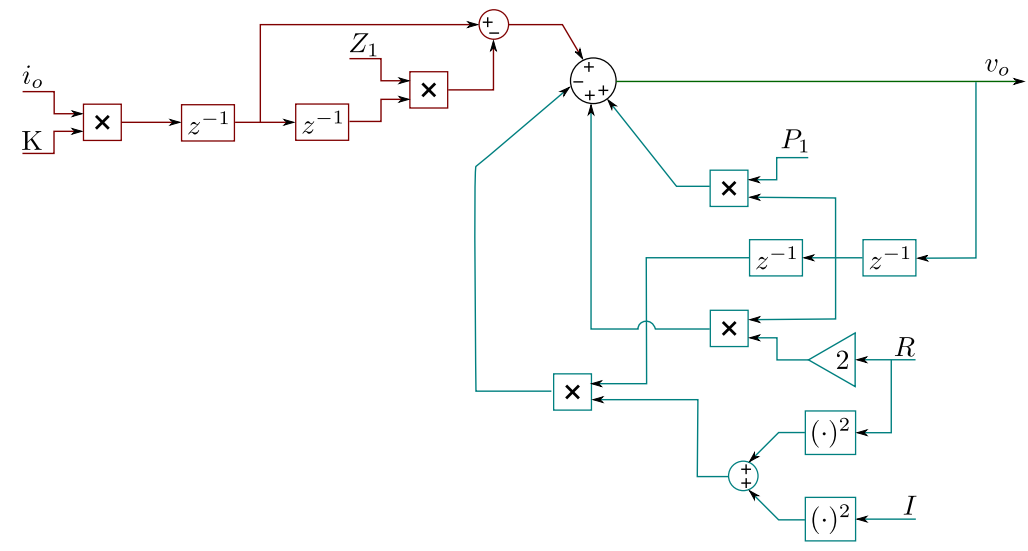

Figure 5.9: Block diagram of the parameter varying transfer function with for the synchronous buck converter with double-loop control with current saturation.

\subsection{Voltage-controlled synchronous buck converter with double-loop control and current saturation case}

In Section 3.2 it was shown that the polytopic model was able to reproduce the behavior of converters with different operation modes, but not the transitions from one to the other. In that section it was shown that the Wiener-Hammerstein was the only model able to reproduce with a good accuracy the response of the converter in all conditions. In Section 4.4 it was shown that the polytopic model with dynamic weighting functions was able to have a good estimation of the transition from voltage to current mode by setting the operating point of the output voltage of the model in current mode to the value that the model in voltage mode has in the moment of the transition. However, this approach is not possible for the transition from current to voltage mode because the latter model regulates the output voltage, so the nominal operating point cannot be modified. In this section a parameter varying transfer function model will be designed and compared with the polytopic model with dynamic weighting functions.

First, the transfer functions of the output impedance in voltage and current mode identified (see Table 3.5) are represented using the z-transform:

$$
\begin{gathered}
Z^{v}(z)=\frac{-12.53 e-3(z-1)}{(s-0.9973+0.0156 i)(s-0.9973-0.0156 i)} \\
Z^{i}(z)=\frac{-11.3 e-3}{z-1}
\end{gathered}
$$

Second, the characteristic filter used in the previous chapter for the polytopic model is represented using the z-transform:

$$
\tau(z)=\frac{12.578 e-5(z+0.9983)}{(s-0.9973+0.0156 i)(s-0.9973-0.0156 i)}
$$

The parameter varying transfer function that is able to account for the output impedance in both modes is depicted in Fig. 5.9. Finally, the conditions to change from voltage to current mode are the same as the ones used in Fig. 4.8, but instead of switching from small-signal models, the values of the parameter varying transfer function are modified. A block diagram of the complete model is depicted in Fig. 5.10. 


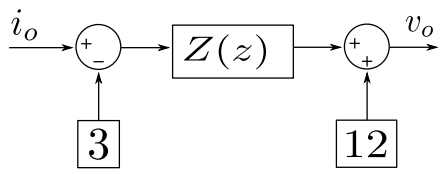

\section{Load}

\section{Models}
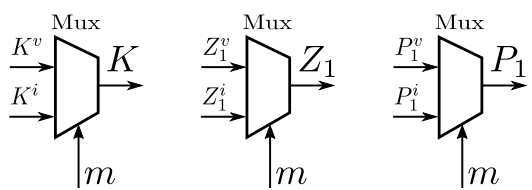
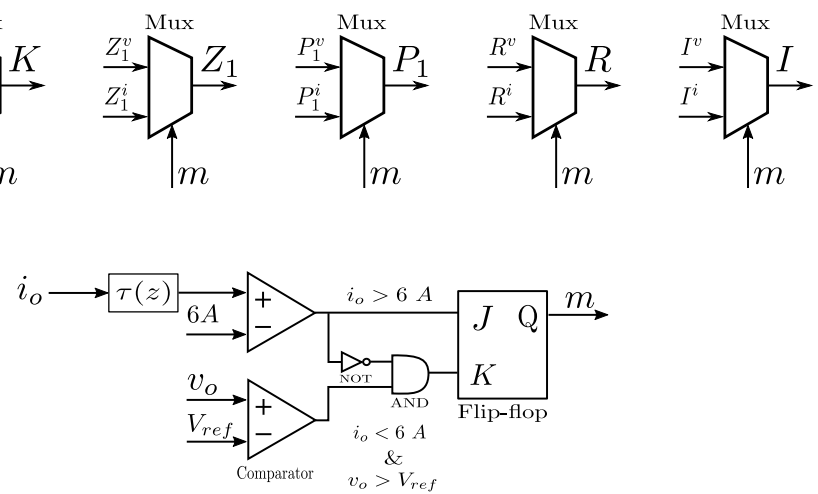

Figure 5.10: Block diagram of the parameter varying transfer functions model with DWF for the case of a voltage-controlled buck converter with a double-loop control and current saturation.

The performance of this model is compared with the switching model and the polytopic model with dynamic weighting functions and a variable operating point for the output voltage of the small-signal model in current mode, which was the most accurate model in the previous chapter (Section 4.4). The result is shown in Fig. 5.11. The converter is initially delivering an output current of $I_{o}=1 \mathrm{~A}$, so the converter is working in voltage mode. At time $t=1 \mathrm{~ms}$ a resistor is connected in parallel with the load increasing the output current to $I_{o}=3 \mathrm{~A}$. As this value is lower than the current limit the converter is still working in voltage mode and both models represent its dynamic behavior correctly.

At time $t=2 \mathrm{~ms}$ another resistor is connected in parallel with the load, increasing the output current to a value above the current limit $I_{O}=6 \mathrm{~A}$. This load step leads the converter to make a transition from voltage to current mode. As detailed in previous chapters, the converter does not switch from voltage to current mode instantly, it starts the transition with the dynamic of the voltage mode and, when the inductor current reaches the current limit, it starts behaving with the dynamic of the current mode. This effect can be approximated accurately with the use of the dynamic weighting function. The parameter varying transfer function does not need to use a variable reference for the output voltage, because it naturally uses the past values of the input and output signals. Therefore, both models are able to represent this transition neatly. At time $t=3 \mathrm{~ms}$ another resistor is connected in parallel with the load, leading the converter to reduce its output voltage, because of the current limitation. It can be seen that both models represent the dynamic response of the converter in current mode accurately.

Finally, at time $t=4 \mathrm{~ms}$ the last two resistors are disconnected and the converter performs a transition from current to voltage mode. As it has been described before, the converter must restore the output voltage level so the maximum current is maintained until the output voltage reaches $V_{o}=12 \mathrm{~V}$. Once the output voltage is restored the converter starts working in voltage mode. This effect is taken into account in the condition to make 

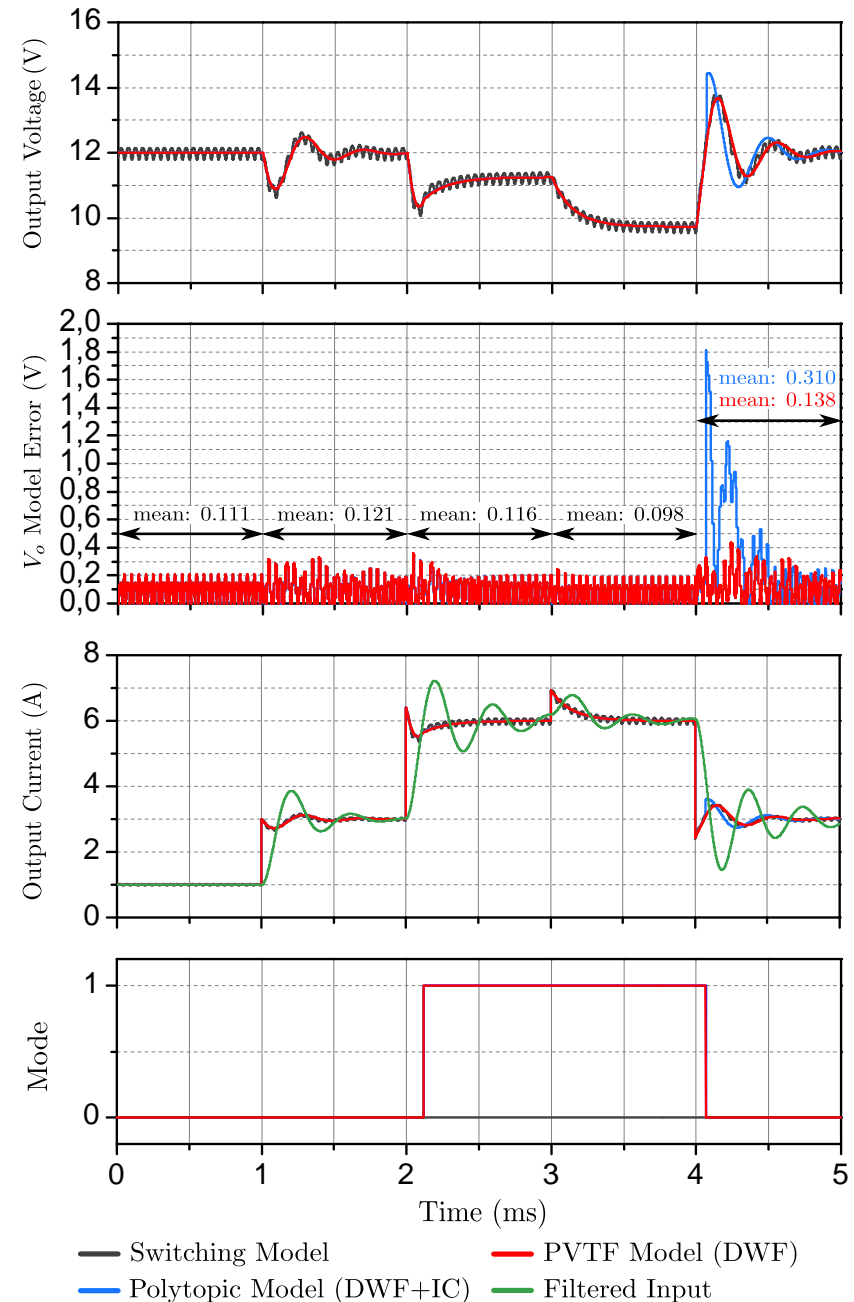

Figure 5.11: Comparison between the switching model and the dynamic weighting functions plus a variable initial condition of the output voltage for the model in current mode and the parameter varying transfer functions (PVTF) model with dynamic weighting functions for the case of a voltage-controlled synchronous buck converter with a double-loop control.

the transition from current to voltage mode. Both models have an accurate result while the converter is in current mode, but in the moment of transition the polytopic model switches to the small-signal model identified in voltage mode, which has a different initial condition. This leads to a discontinuity in the response of the model and, hence, to a poor estimation of the dynamic behavior of the converter during the transition. On the other hand, the parameter varying transfer function model takes advantage of its inherent capability of taking into account the past values of the input and output signals and it just changes the parameters of the transfer function, which affect the dynamic behavior of the model from the initial conditions in which the transition takes place. Consequently, the performance of this model is excellent in all conditions.

The error between the switching model and the blackbox models considered also represented in Fig 5.11, where it can be seen that the parameter varying transfer functions model keep the error very close to the switching ripple level in all the transitions.

Finally, in Fig. 5.12 the different measures of the overall error of the models in presented. This time, a reduction of $76 \%$ of the maximum error is achieved with the PVTF model. 


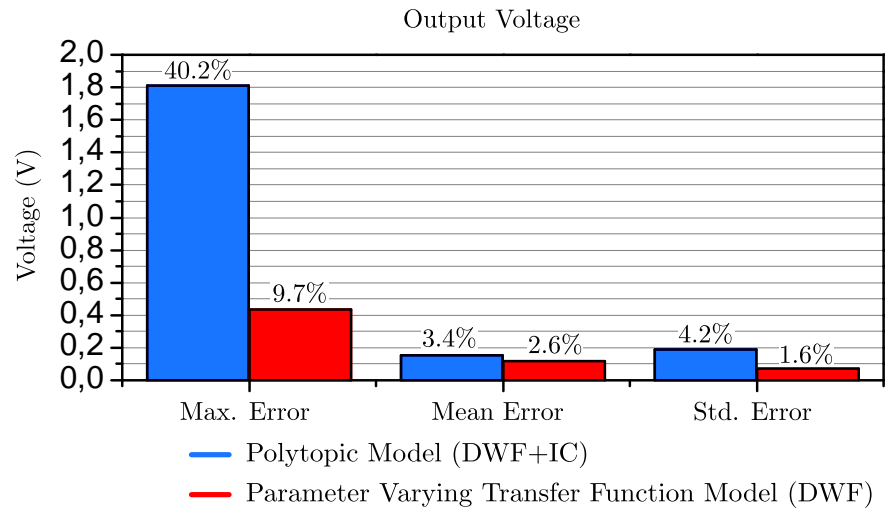

Figure 5.12: Details about the performance of the dynamic weighting functions plus a variable initial condition of the output voltage for the model in current mode and the parameter varying transfer functions (PVTF) model with dynamic weighting functions for the case of a voltage-controlled synchronous buck converter with a double-loop control.

\subsection{Conclusions}

In Chapter 4 the polytopic model with dynamic weighting functions was proposed in order to improve the performance of the polytopic model with the classical static weighting function for large and sharp variations in the input variables of the model. It was shown that its performance for converters with soft variation in its dynamic response the model was able to improve the estimation compared with the polytopic model with static weighting functions. However, it was also shown that when the variation in the dynamic response of the converter was considerable, the performance of the polytopic model with dynamic weighting functions was not acceptable.

In this chapter the reason for this inaccurate result was described. In the case of converters with highly variable dynamics, the use of a weighted combination of small-signal models working in parallel can lead to a problem of initial conditions, i.e. a transition from fast to slow models or vice versa creates discontinuities in the model response, because they do not take into account the values of the output variables in the moment of the transition. In order to account for this effect a new modeling approach is proposed, the parameter varying transfer functions model. In this approach, instead of applying the weighting functions to the output of the individual small-signal models, it is applied to the parameters of the transfer function of a single small-signal model. Therefore, what it is changed is the dynamic of the transfer function, which takes into account the past values of the input and output signals.

The two illustrative examples studied in previous chapters corresponding to converters with highly variable dynamic behaviors, i.e. a buck converter in DCM and CCM and a buck converter with different operation modes, were used to validate the proposed modeling approach. The results endorse the hypothesis that the actual behavior of the converter can be better reproduced with transfer functions that change their parameters than with a weighted combination of small-signal models. The parameter varying transfer function model was able to achieve a high accuracy for these converters in all the scenarios under consideration.

Finally, the examples described are relevant for dc microgrids because they take into account the main characteristics of this kind of systems, i.e. large variation of the operating points of the variables of the system and different operation modes of the converters, that can be caused by current limitations in the control to protect the devices or the DBS control strategy that protects the system from overloading in case the microgrid is not able 
to absorb all the energy produced by the sources (see Section 2.1.2).

The proposal of using parameter varying transfer functions models, instead of polytopic models, with dynamic weighting functions in order to obtain very accurate blackbox models for highly nonlinear EPCs is an important contribution of this thesis. 


\section{Large-signal blackbox stability analysis}

In

In Section 2.4 the different stability analysis approaches to study the interconnection of EPCs were reviewed. They can be classified in small and large signal methods. The most widely used small-signal stability criteria are based on the analysis of the minor loop gain. This method assures that, provided that the EPCs are individually stable, the stability of the interconnection can be studied by just applying the Nyquist criterion to the minor-loop gain, which consists of the output impedance of the source converter and the input admittance of the load converter (considering a constant direction of the power flow). Various criteria have been proposed to define the robustness of the stability, namely Middlebrook, gain and phase margin, ESAC, opposing argument, and Maximum Peak Criterion (MPC) criteria. These methods define a forbidden region within the s-plane that the minor-loop gain must avoid to assure a stable behavior of the interconnected system. All these immittance-based methods can be readily applied using blackbox techniques, as the information needed is available, for instance in the G-parameters model (see Section 2.3.1).

However, the immittance-based criteria are subjected to the small-signal limitations, i.e. the information obtained by this analysis will be accurate for small-signal perturbations around the local model considered. In case the converters have nonlinear behaviors, it is unknown the range of operating points, around the one considered, for which the result obtained is valid. The Lyapunov method can be applied in order to obtain an estimation of the region of attraction around a particular equilibrium point. In dc microgrids a typical example is the CPL. This effect is produced when an EPC supplies another tightly regulated converter connected to a resistive load. In this situation the Point-of-Load (POL) converter works as a constant power load, which is seen by the source converter as a negative impedance, this effect is depicted in Fig. 6.1. This negative impedance behavior has a well-known destabilizing effect, hence many works have been devoted to the analysis of this kind of system [115-117].

In this chapter a first approach towards a blackbox large signal stability analysis will be studied. With this objective a large signal analysis of a series connection of a source converter supplying a POL converter connected to a resistive load, as the one depicted in Fig. 6.2, will be assessed from a blackbox perspective. The goal is to estimate the ROA of an equilibrium point using the local models that can be obtained using identification techniques. In order to have a reference for comparison, the example shown in [118] will be 


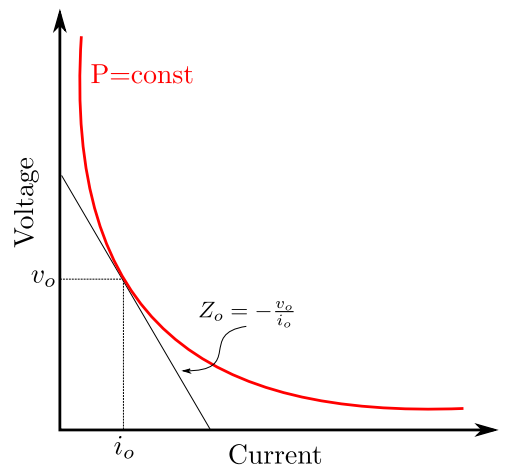

Figure 6.1: Negative impedance behavior of regulated POL converters.

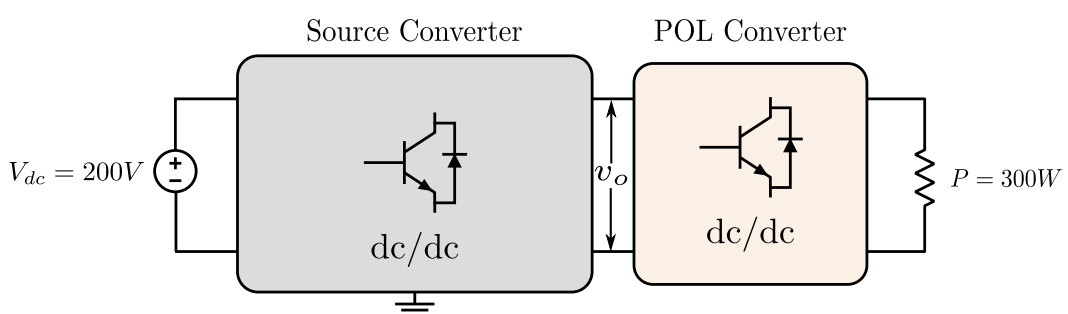

Figure 6.2: Scheme of a source converter supplying a POL converter connected to a resistive load.

used. The goal is to achieve the same ROA obtained with analytical method, i.e. with the knowledge of the equations of the system and the value of its parameter, using a blackbox approach. The results presented in this chapter are based on the paper [119] published by the authors of this thesis.

\subsection{Application of the Lyapunov method to blackbox polytopic structures}

In Section 2.4.2 a review of different methods to obtain Lyapunov candidates for EPCs was presented. From this analysis it was justified that the multi-model approach is very suitable for blackbox stability analysis as it does not rely on the knowledge of the internal architecture of the converters and it is based on local models, which can be obtained using identification methods. This method consists in selecting a collection of local models around an equilibrium point and applying the Lyapunov stability condition. If a Lyapunov candidate that complies the condition for all local models exists, the region defined by the operating points considered belong to the ROA of this equilibrium point, therefore the objective is to find the biggest set of operating points around the equilibrium point that complies with the Lyapunov condition. As the Lyapunov candidate can be regarded as a measure of the energy of the system, the idea behind these methods is that if the system loses energy in every local model around the equilibrium point, it will eventually end in the equilibrium point.

This method requires the state-space equations of the system. Using a blackbox approach, a state-space representation can be obtained, however the main difficulty is that the state variables of the system are unknown. Therefore, the equations obtained are just a mathematical expression, where the only identifiable physical variables are the inputs and outputs of the system. The solution proposed to overcome this problem is to make a similarity transformation to the state-space representation such that the output matrix is equal to the identity matrix. With this transformation a state-space representation is obtained where 
the state variables are the outputs of the system. Therefore, the ROA obtained will be referred to the output variables, which are the only information available about the system. This solution is valid for systems with equal or less state variables than the number of output signals, or systems that can be reduced to an order equal to the number of outputs. The similarity transformation can be expressed as:

$$
\begin{aligned}
\dot{x} & =C_{i} A_{i} C_{i}^{-1} x+C_{i} B_{i} u \\
y & =C_{i} C_{i}^{-1} x+D_{i} u
\end{aligned}
$$

where the subindex $i$ refers to the local models obtained around different operating points.

\subsubsection{Coincident polytopic models}

Another difficulty of using a blackbox approach is how to obtain coincident polytopic models. Coincident polytopic models refer to local models around the equilibrium point that share the same equilibrium point. In case this condition is not met, the idea in which this method is based, i.e. if the system loses energy in each local model it will end in the equilibrium point, will not mean that the system is stable, because if there are more than one equilibrium point, the system could oscillate among them.

If the homogeneous system is considered, the relationship between local models and coincident models is:

$$
A_{n}\left(x_{o}-x_{e}\right)=\left(A_{o} x_{o}-A_{e} x_{e}\right) \frac{1}{n}
$$

where $n$ is the order of the nonlinear term. Isolating the term $A_{n}$ the following equation is derived:

$$
A_{n}=\left(A_{o} x_{o}-A_{e} x_{e}\right) \frac{1}{n\left(x_{o}-x_{e}\right)}
$$

In order to prove the relation (6.3), it is considered a general system with a nonlinearity of the kind:

$$
\dot{x}=x^{n}+C
$$

where the constant term can be related with the equilibrium point, imposing the condition that its derivative is zero:

$$
\dot{x}=x^{n}-x_{e}^{n}
$$

It is possible to define its state space as:

$$
\dot{x}=\frac{x^{n}-x_{e}^{n}}{x-x_{e}}\left(x-x_{e}\right)
$$

therefore its coincident model around the operating point $x_{o}$ is:

$$
\dot{x}=\frac{x_{o}^{n}-x_{e}^{n}}{x_{o}-x_{e}}\left(x-x_{e}\right)
$$


Notice how the state matrix:

$$
A_{n}=\frac{x_{o}^{n}-x_{e}^{n}}{x_{o}-x_{e}}
$$

is variable and depends on the operating point considered, whereas the equilibrium point is always constant and equal to $x_{e}$.

The linearization of the system (6.5) around an operating point, $x_{o}$, would be:

$$
y^{\prime}(x)=n x_{o}^{n-1} x
$$

therefore the local state matrix is:

$$
A_{o}=n x_{o}^{n-1}
$$

which is what it can be identified from a perturbation around this operating point.

Finally, using (6.3), $A_{n}$ is obtained:

$$
A_{n}=\left(n x_{o}^{n-1} x_{o}-n x_{e}^{n-1} x_{e}\right) \frac{1}{n\left(x_{o}-x_{e}\right)}=\frac{x_{o}^{n}-x_{e}^{n}}{x_{o}-x_{e}}
$$

The system with CPL behavior will have a nonlinearity of the kind:

$$
\dot{x}=\frac{1}{x}+C
$$

where the constant term can be related with the equilibrium point, as it was described for the general case:

$$
\dot{x}=\frac{1}{x}-\frac{1}{x_{e}}
$$

rearranging terms the following expression is obtained:

$$
\dot{x}=\frac{-\left(x-x_{e}\right)}{x x_{e}}
$$

Therefore, the state matrix of the system is:

$$
A_{n}=\frac{-1}{x x_{e}}
$$

Using a blackbox approach, performing perturbations around different equilibrium points, a linearization around the equilibrium points considered are obtained referred to the origin. For this nonlinearity the following expression would be obtained:

$$
\dot{x}=\frac{-1}{x_{o}^{2}} x
$$




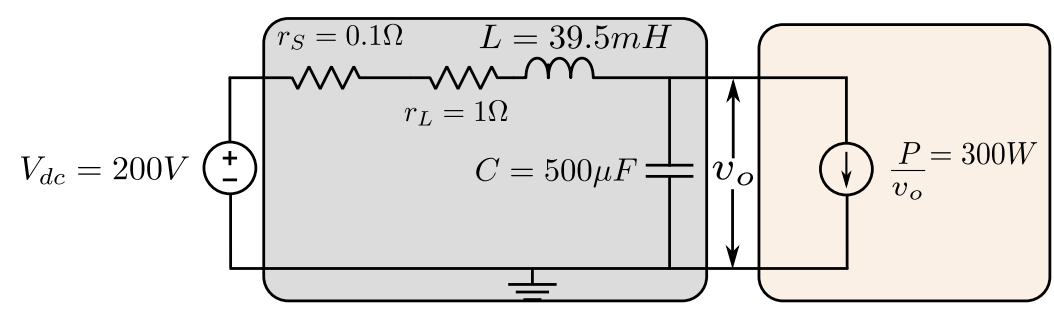

Figure 6.3: Scheme of the simplified circuit representing a cascade connection of a source converter supplying a POL converter that has a CPL behavior.

where $x_{o}$ corresponds to the operating point where the identification was performed. Applying (6.3) the following equation is obtained:

$$
\left(-\frac{1}{x_{o}^{2}} x_{o}+\frac{1}{x_{e}^{2}} x_{e}\right) \frac{1}{(-1)\left(x_{o}-x_{e}\right)}=\frac{x_{o}-x_{e}}{x_{o} x_{e}} \frac{-1}{x_{o}-x_{e}}=\frac{-1}{x x_{e}}
$$

which is equal to $A_{n}$.

\subsection{Constant power load example}

The method detailed above will be applied to the example shown in [118], so the ROA obtained analytically can be compared with the one obtained using a blackbox approach. The system considered is depicted in Fig. 6.3, where $r_{s}$ represents the output impedance of the source, $r_{L}, L$, and $C$ are the parameters of a buck converter, which is simplified by its output filter, and $P$ is the power delivered to the load. The POL converter is simplified by a CPL. is:

First the analytical model will be derived. The autonomous state-space representation

$$
\begin{aligned}
& \dot{x}_{1}=-\frac{r_{s}+r_{L}}{L} x_{1}-\frac{1}{L} x_{2}+\frac{1}{L} V_{d} c \\
& \dot{x}_{2}=\frac{1}{C} x_{1}-\frac{P}{C x_{2}}
\end{aligned}
$$

where $x_{1}$ and $x_{2}$ are the inductor current and the capacitor voltage respectively. In order to see the behavior around the equilibrium point, the change of coordinates $\tilde{x}_{i}=x_{i}-x_{e}$ is implemented, so the system becomes:

$$
\begin{aligned}
& \dot{\tilde{x}}_{1}=-\frac{r_{s}+r_{L}}{L} \tilde{x}_{1}-\frac{1}{L} \tilde{x}_{2} \\
& \dot{\tilde{x}}_{2}=\frac{1}{C} \tilde{x}_{1}+\frac{P}{C x_{e}} \frac{\tilde{x}_{2}}{\tilde{x}_{2}+x_{e}}
\end{aligned}
$$

Therefore the $A_{n}$ matrix is:

$$
A_{n}=\left(\begin{array}{cc}
-\frac{r_{s}+r_{L}}{L} & -\frac{1}{L} \\
\frac{1}{C} & \frac{P}{C x_{2 e} x_{2 o}}
\end{array}\right)
$$


Using a blackbox approach, a linearization of the state matrix (6.18) will be identified:

$$
A_{\text {lin }}=\left(\begin{array}{cc}
-\frac{r_{s}+r_{L}}{L} & -\frac{1}{L} \\
\frac{1}{C} & \frac{P}{C x^{2}}
\end{array}\right)
$$

Now using (6.3) the coincident model is obtained:

$$
\begin{aligned}
A_{n}= & \left(\left(\begin{array}{cc}
-\frac{r_{s}+r_{L}}{L} & -\frac{1}{L} \\
\frac{1}{C} & \frac{P}{C x_{2 o}^{2}}
\end{array}\right)\left(\begin{array}{c}
x_{1 o} \\
x_{2 o}
\end{array}\right)-\left(\begin{array}{cc}
-\frac{r_{s}+r_{L}}{L} & -\frac{1}{L} \\
\frac{1}{C} & \frac{P}{C x_{2 e}^{2}}
\end{array}\right)\left(\begin{array}{c}
x_{1 e} \\
x_{2 e}
\end{array}\right)\right) . \\
& \cdot\left(\begin{array}{ll}
\frac{1}{x_{1 o}-x_{1 e}} & \frac{1}{x_{2 o}-x_{2 e}} \\
\frac{1}{x_{1 o}-x_{1 e}} & \frac{-1}{x_{2 o}-x_{2 e}}
\end{array}\right)
\end{aligned}
$$

which results in:

$$
A_{n}=\left(\begin{array}{cc}
-\frac{r_{s}+r_{L}}{L}\left(x_{1 o}-x_{1 e}\right) & -\frac{1}{L}\left(x_{2 o}-x_{2 e}\right) \\
\frac{1}{C}\left(x_{1 o}-x_{1 e}\right) & \frac{P}{C}\left(\frac{1}{x_{2 o}}-\frac{1}{x_{2 e}}\right)
\end{array}\right)\left(\begin{array}{cc}
\frac{1}{x_{1 o}-x_{1 e}} & \frac{1}{x_{2 o}-x_{2 e}} \\
\frac{1}{x_{1 o}-x_{1 e}} & \frac{-1}{x_{2 o}-x_{2 e}}
\end{array}\right)
$$

and finally:

$$
A_{n}=\left(\begin{array}{cc}
-\frac{r_{s}+r_{L}}{L} & -\frac{1}{L} \\
\frac{1}{C} & \frac{P}{C x_{2 e} x_{2 o}}
\end{array}\right)
$$

which is the same as the one obtained from the nonlinear equations (6.20).

\subsubsection{Blackbox model}

The two converters of the system shown in Fig. 6.3 will be identified individually and then combined in order to obtain the state space representation of the whole system. The first converter is an unregulated buck converter so, as showed in Section 3.1.1, a G-parameters model can represent its dynamic accurately. Besides, it has been simplified with its output filter, following the approach shown in [118] and justified by the mentioned linear behavior of this kind of converters. The POL converter will have a nonlinear response due to the CPL behavior of the converter. Actually, it has been simplified with a CPL, also following the approach of the cited work. This simplification approximates the response of the converter by its low frequency behavior, where the nonlinearity is reflected. However, it is important to notice that an additional impedance based stability analysis should be also performed in order to assure that there is no high frequency dynamic interaction between the source and 

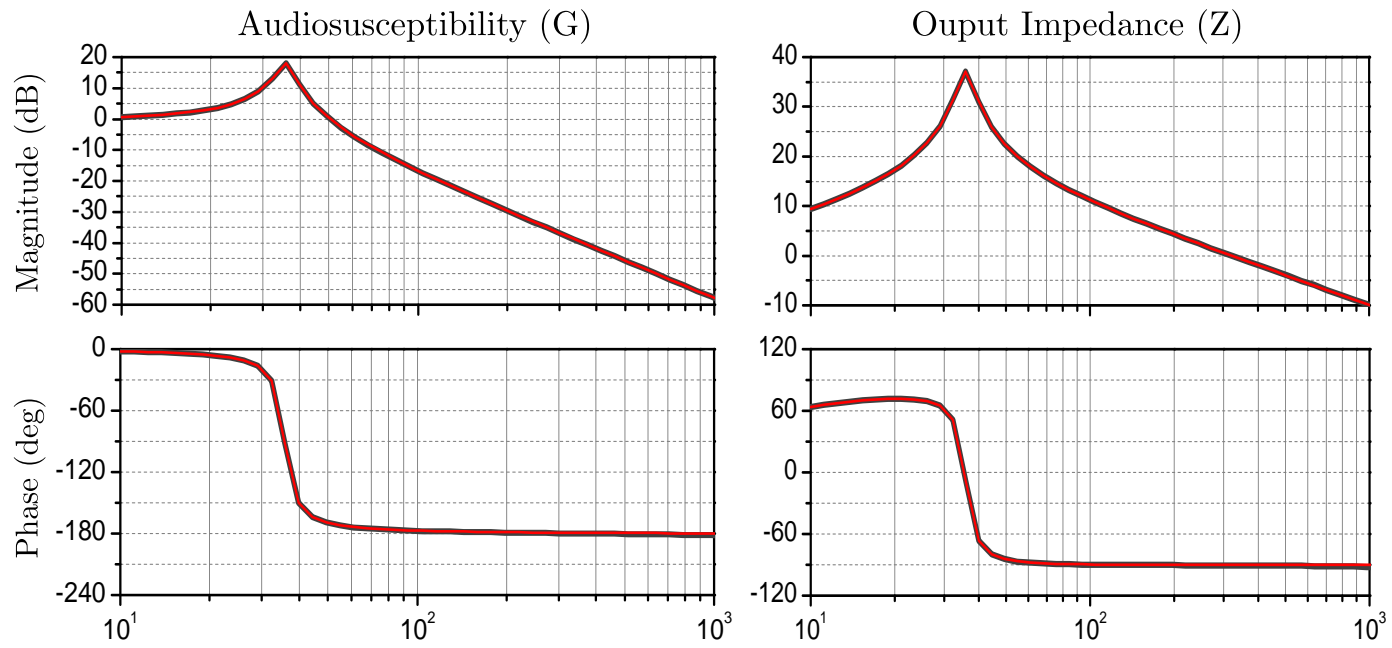

Frequency $(\mathrm{Hz})$
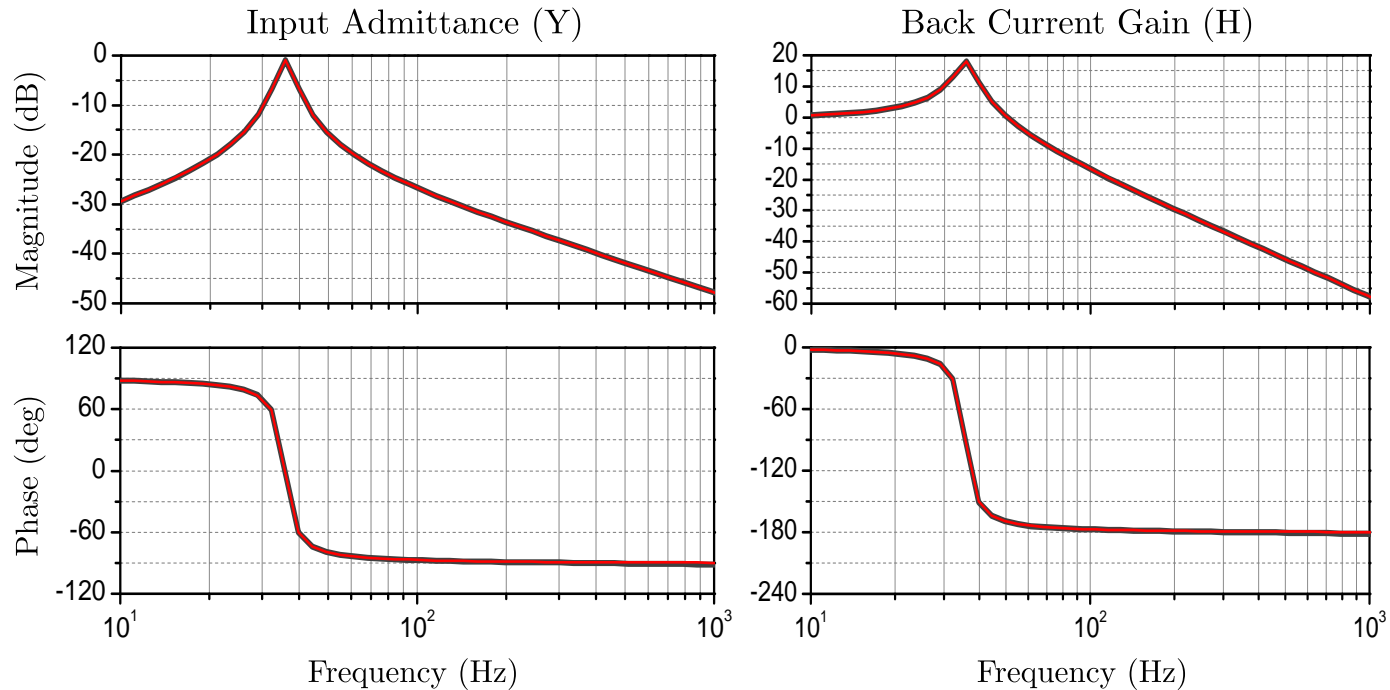

— Switching Model — Identified Model

Figure 6.4: Comparison between the frequency response of the source converter and the identified transfer functions.

load converters. Therefore, this large-signal stability analysis would be part of a second stage of the stability assessment.

The frequency response of the simplified source converter, when it is subjected to the tests to identify the G-parameters model, and the comparison with the identified transfer functions are presented in Fig. 6.4. The expressions of the identified transfer functions are shown in Table 6.1.

Regarding the simplified POL converter, its model is reduced to the input admittance. This simplification would correspond to the low-frequency response of a POL converter with constant output current. The expression of the input admittance could be identified from measurements, but it actually can be simplified with the following expression: 
Table 6.1: Parameters of the identified transfer functions of the source converter, corresponding to a unregulated synchronous buck converter simplified with its output filter.

\begin{tabular}{|c||c|}
\hline G-parameter & Transfer function \\
\hline \hline$G(s)$ & $\frac{50635}{s^{2}+28.0037 s+50629}$ \\
\hline$Z(s)$ & $\frac{2001.3 s+55919}{s^{2}+27.9872 s+50646}$ \\
\hline$Y(s)$ & $\frac{25.3199 s+4.3303}{s^{2}+27.9987 s+50627}$ \\
\hline$H(s)$ & $\frac{50677}{s^{2}+27.9883 s+50647}$ \\
\hline
\end{tabular}

$$
Y_{P O L}=-\frac{P}{v_{o}^{2}}
$$

Using this expression, the local models for different values of the output voltage are presented in Table 6.2. However, in order to confirm this approximation, the CPL has been tested and the identified input admittance is also shown in the Table. The resulting transfer function is of first order, where the pole and the zero are in very high frequencies, so they are actually negligible. The dc gain of the transfer function is coincident with the approximation performed.

Following the approach shown in Appendix A, the input-output expression of the interconnected system can be represented as:

$$
\left(\begin{array}{c}
v_{o} \\
i_{i}
\end{array}\right)=\left(\begin{array}{c}
\frac{G(s)}{1+Z(s) Y_{P O L}^{i}(s)} \\
Y(s)+\frac{H(s) G(s) Y_{P O L}^{i}(s)}{1+Z(s) Y_{P O L}^{i}(s)}
\end{array}\right) v_{i}
$$

where the superscript $i$ represents the different operating points where $Y_{P O L}$ has been identified. The evaluation of (6.26) using the identified transfer functions presented in Table 6.1 and Table 6.2 is shown in Table 6.3. Actually, the evaluation of the interconnected model results in high order transfer functions, so model order reduction methods have been applied to reduce the order of the transfer functions. In particular, the Matlab functions balreal and modred have been applied for this purpose. The balreal function provides the Hankel singular values of the states, which correspond to a measure of their influence in the dynamic behavior of the system. Therefore, states with small values can be removed using the function modred.

The input-output models presented in Table 6.3 can be easily represented in the state space using the controllable canonical form. If a generic transfer function is considered:

$$
T(s)=\frac{b_{0} s^{n}+b_{1} s^{n-1}+\ldots+b_{n-1} s+b_{n}}{s^{n}+a_{1} s^{n-1}+\ldots+a_{n-1} s+a_{n}}
$$


Table 6.2: Evaluation of the input admittance of the simplified POL converter for different values of the output voltage of the source converter and a constant output power, $P=300 \mathrm{~W}$.

\begin{tabular}{|c||c||c|}
\hline Output voltage & $Y_{P O L}$, identified & $Y_{P O L}$, simplified \\
\hline \hline$V_{o}=100 \mathrm{~V}$ & $\frac{0.0349 s-41756}{s+1391610}$ & -0.03 \\
\hline$V_{o}=120 \mathrm{~V}$ & $\frac{0.0228 s-38138}{s+1830582}$ & -0.02083 \\
\hline$V_{o}=150 \mathrm{~V}$ & $\frac{0.0162 s-19001}{s+1425025}$ & -0.013 \\
\hline$V_{o}=200 \mathrm{~V}$ & $\frac{0.0109 s-120550}{s+16074595}$ & -0.0075 \\
\hline$V_{o}=250 \mathrm{~V}$ & $\frac{0.0058 s-6814.3}{s+1419658}$ & -0.0048 \\
\hline$V_{o}=280 \mathrm{~V}$ & $\frac{0.0041 s-6979}{s+1823681}$ & -0.0038 \\
\hline$V_{o}=300 \mathrm{~V}$ & $\frac{0.0042 s-4843}{s+1452917}$ & -0.0033 \\
\hline
\end{tabular}

its state-space matrices in the controllable canonical form are:

$$
\begin{gathered}
A=\left[\begin{array}{ccccc}
0 & 1 & 0 & \cdots & 0 \\
0 & 0 & 1 & \cdots & 0 \\
: & : & : & \ddots & : \\
0 & 0 & 0 & \cdots & 1 \\
-a_{n} & -a_{n-1} & -a_{n-2} & \cdots & a_{1}
\end{array}\right] \quad B=\left[\begin{array}{c}
0 \\
0 \\
: \\
0 \\
1
\end{array}\right] \\
C=\left[\left(b_{n}-a_{n} b_{o}\right)\left(b_{n-1}-a_{n-1} b_{o}\right) \cdots\left(b_{2}-a_{2} b_{o}\right)\left(b_{1}-a_{1} b_{o}\right)\right] \quad D=b_{0}
\end{gathered}
$$

The state-space representation of the models around different operating points are obtained using the relation shown in (6.28). Next, the similarity transformation shown in (6.1) is applied in order to relate the state variables with the outputs of the system. The resulting state matrices of the different local models are presented in Table 6.4, which correspond with the evaluation of (6.21) around the different operating points. Finally, applying (6.3) to each local state matrix, the coincident state matrices can be derived, which are also presented in Table 6.4. It can be confirmed that the expression of the identified local coincident state matrices are equivalent to the evaluation of the analytical expression shown in $(6.20)$.

\subsubsection{Linear stability analysis}

The linear stability analysis of the interconnection of the source and load converters can be performed applying the Nyquist criterion to the minor-loop gain. Using the identified transfer function of the output impedance of the source converter (Table 6.1) and the input admittance of the POL converter (Table 6.2) for the different values of the output voltage, 
Table 6.3: Parameters of the interconnected system using the identified G-parameters models of the converters.

\begin{tabular}{|c|c|c|}
\hline Output Voltage & \multicolumn{2}{|c|}{ Input-output Model } \\
\hline$v_{o}=100 \mathrm{~V}$ & $\frac{1}{s^{2}-32.02 s+4.89 e 4}$ & $\begin{array}{c}-23.3 e-3 s+5063.8 \\
25.3 s-1518.7\end{array}$ \\
\hline$v_{o}=120 \mathrm{~V}$ & $\frac{1}{s^{2}-13.7 s+4.95 e 4}$ & $\begin{array}{c}-33.6 e-3 s+5061.5 \\
25.3 s-1052.7\end{array}$ \\
\hline$v_{o}=150 \mathrm{~V}$ & $\frac{1}{s^{2}+1.3 s+4.99 e 4}$ & $\begin{array}{c}-44.1 e-3 s+5059.1 \\
25.3 s-672.11\end{array}$ \\
\hline$v_{o}=200 \mathrm{~V}$ & $\frac{1}{s^{2}+12.99 s+5.02 e 4}$ & $\begin{array}{c}-21.7 e-3 s+5066.1 \\
25.3 s-380.81\end{array}$ \\
\hline$v_{o}=250 \mathrm{~V}$ & $\frac{1}{s^{2}+18.4 s+5.04 e 4}$ & $\begin{array}{c}-14.3 e-3 s+5067.6 \\
25.3 s-243.88\end{array}$ \\
\hline$v_{o}=280 \mathrm{~V}$ & $\frac{1}{s^{2}+20.35 s+5.04 e 4}$ & $\begin{array}{c}-11.5 e-3 s+5068 \\
25.3 s-194.44\end{array}$ \\
\hline$v_{o}=300 \mathrm{~V}$ & $\frac{1}{s^{2}+21.34 s+5.04 e 4}$ & $\begin{array}{c}-10.1 e-3 s+5068.1 \\
25.3 s-169.39\end{array}$ \\
\hline
\end{tabular}

the Nyquist diagram of the minor-loop gain can be represented for different operating points. Fig. 6.5 shows the Nyquist diagram of the interconnected system for three different values of the output voltage $v_{o}=\{100,150,300\} \mathrm{V}$. According to this analysis the system is close to instability when the output voltage is $v_{o}=150 \mathrm{~V}$. For values of the output voltage lower than this value the system is instable, whereas for values of the output voltage higher than this value the margin of stability increases.

The conclusion that can be extracted from this result is that beyond $v_{o}=150 \mathrm{~V}$ the system is stable and the equilibrium point has a certain, unknown, region of stability around it. However, this method does not give information about the size of the region of attraction, i.e. how far can the initial conditions be from the equilibrium point and still return to it, or how large can be the perturbations in the input variables without destabilizing the system.

Considering the equilibrium point of the output voltage $v_{o}=200 \mathrm{~V}$, from the Nyquist diagram it would be reasonable to think that, for initial conditions of the output voltage higher than this value, the system should be stable, as the margin of stability increases. Besides, as the nonlinearity is only reflected in the output voltage, this analysis does not consider the effect of the input current, so no conclusions could be extracted about the 
Table 6.4: Parameters of the local state matrices obtained from the transfer functions identified after the similarity transformation.

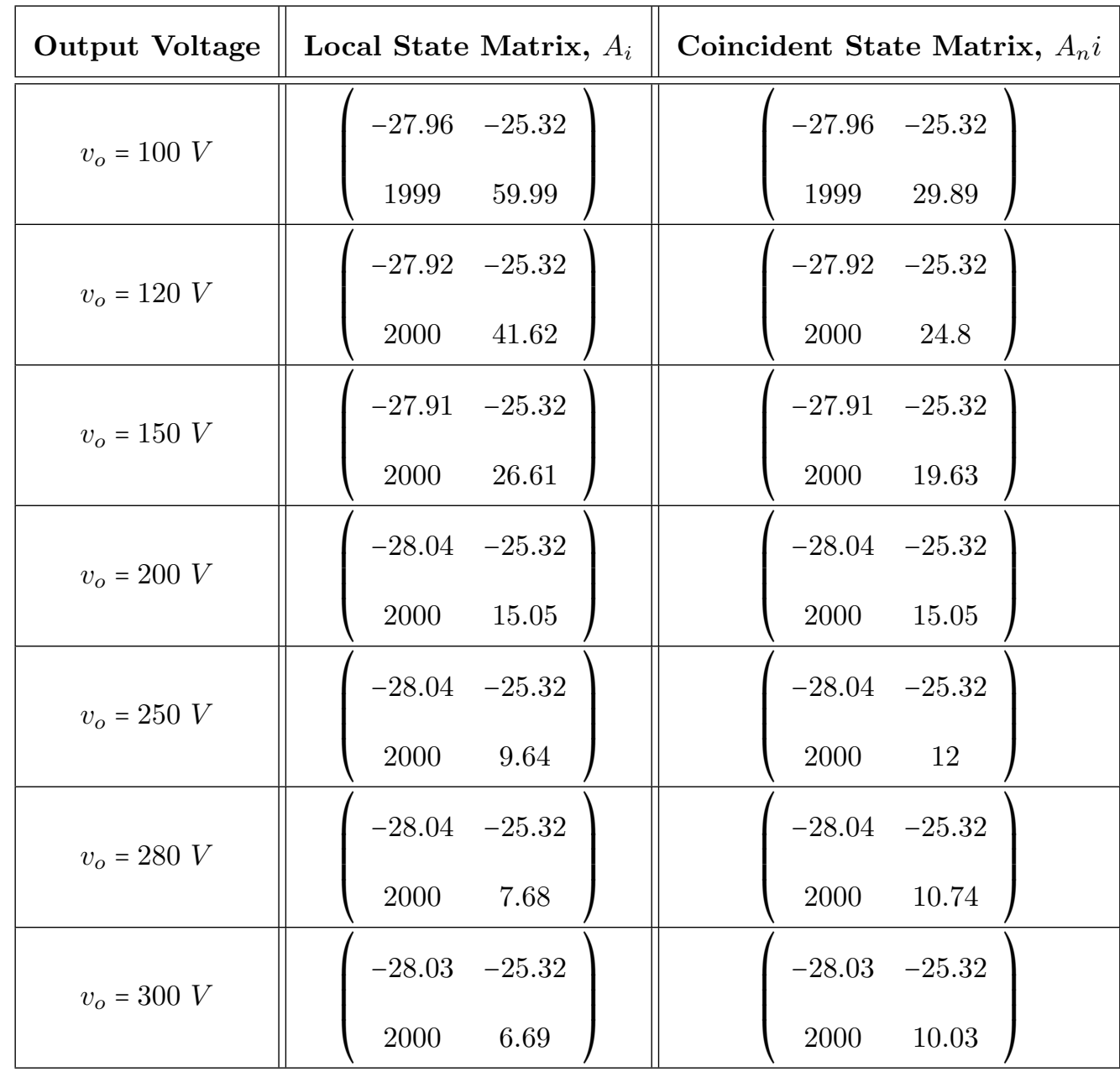

initial conditions of this variable.

\subsubsection{Large signal stability analysis}

Using the local coincident state matrices identified for different values of the output voltage (Table 6.4), the multimodel Lyapunov stability condition can be applied (2.45). This condition corresponds to a LMI problem, which can be solved, for instance, using the Matlab LMI toolbox. The approach is to solve this problem for the matrix obtained in the operating points nearby the equilibrium, $x_{e} \pm \delta$. In case the problem is feasible, operating points further away from the equilibrium are evaluated until the problem becomes unfeasible. The last value of $\delta$ will be a limit of the region of stability around this equilibrium point. In the two-dimensional case, the quadratic Lyapunov candidate described before defines an ellipse (6.29), characterized by the obtained matrix $P$ and a constant that can be evaluated with one of the variables maximum value.

$$
P(1,1) \cdot x_{1}^{2}+P(2,2) \cdot x_{2}^{2}+2 \cdot P(2,1) \cdot x_{1} \cdot x_{2}=a
$$




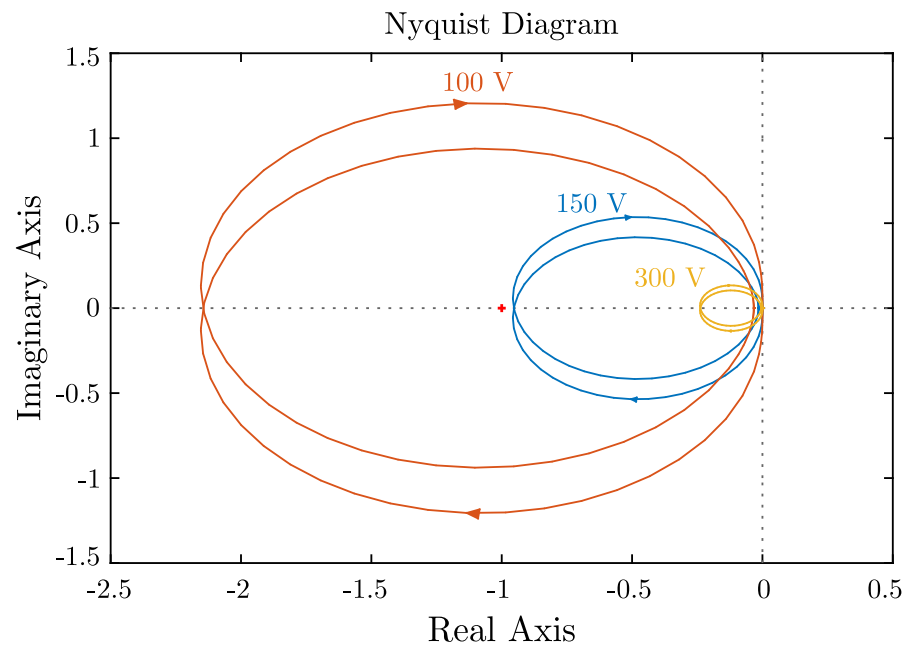

Figure 6.5: Nyquist diagram of the minor-loop gain of the interconnected converter using the different local values of the input admittance of the POL converter.

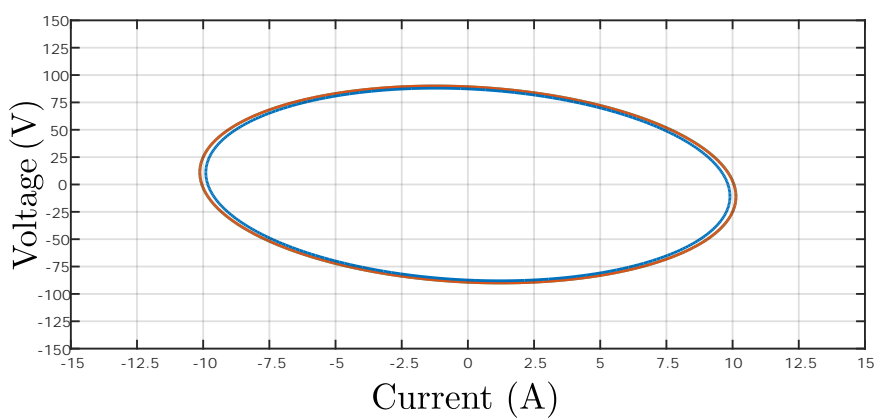

Figure 6.6: Regions of stability around the equilibrium point obtained using the whitebox (red) and blackbox (blue) approaches.

In Fig. 6.6 the region of stability obtained using the proposed blackbox and the analytical methods are shown. The results for this example show that using the proposed method the region of stability is quite similar to the one obtained from the whitebox approach. Therefore, nonlinear stability analysis of this kind of systems could be performed even if no details about the internal architecture of the converters is known, and the results seem to be an accurate approximation of the ones obtained using the described analytical method.

It can be also seen that there is an upper bound for the deviations from the equilibrium point for which the stability can be assured. Using the admittance criterion we were not able to identify this upper bound, therefore if we would have tried to extrapolate a region of stability, the result would be incorrect. In order to validate these results, the response of the system is depicted in Fig. 6.7 with different initial conditions. It is shown that the system becomes unstable when the initial value of the output voltage is $V_{o}=350 \mathrm{~V}$, and that for a corner point in the region of stability, initial conditions $V_{o}=250 \mathrm{~V}$ and $i_{i}=6.5$ $A$, the system is still asymptotically stable. However, as it is well-known, the Lyapunov method can provide conservative results. In [112] the difference between the obtained region of stability is compared with the real one, obtained numerically. 


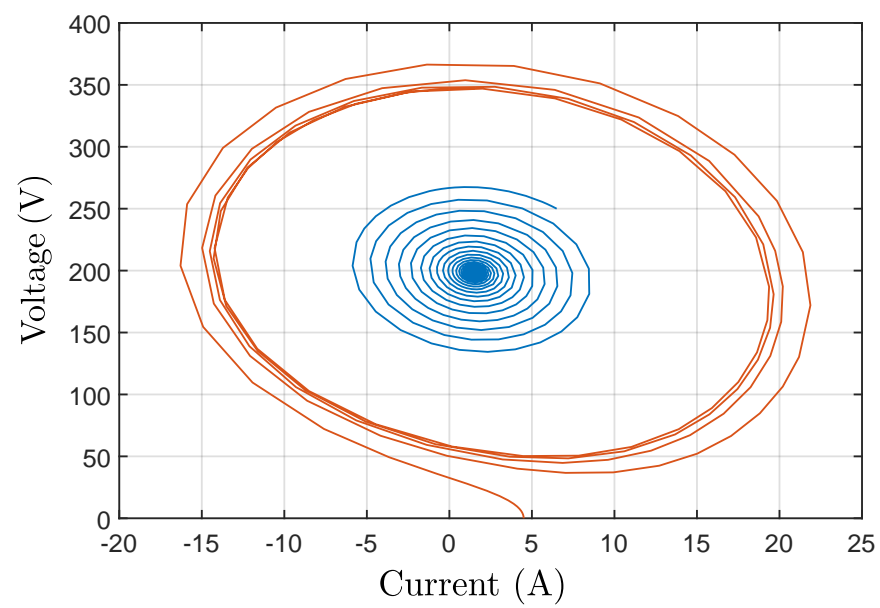

Figure 6.7: State variables response with different initial conditions.

\subsection{Conclusions}

In this chapter a first approach to perform large-signal stability analysis using blackbox models has been proposed. To the best of the author's knowledge, in the literature there are no works that apply nonlinear stability analysis using identified models. The method is based on the identification of local models around different operating points of the different converters involved in the analysis. These models can be interconnected creating a overall model of the interconnected system. This process often requires the use of model order reduction techniques, as it results in artificial high order systems. From the interconnected input-output model the state space representation can be derived.

A similarity transformation is proposed in order to relate the state variables with the output variables of the model, which are known. Therefore, the conclusions extracted will be related to physical variables which provide meaningful information. Besides, it is proposed a method to obtain coincident models from the different local models identified. The local models identified have different equilibrium points, as the operating point of the converter must be varied in order to perform the identification tests. However, in order to perform the multimodel Lyapunov method, the local models must share the same equilibrium point, as it was detailed in this chapter. The methodology to obtain coincident models from local models obtained around different operating points, was mathematically demonstrated for a general nonlinearity system of the kind $x^{n}$, as it is the case of the systems with CPLs, which has a nonlinearity of the kind $x^{-1}$.

In order to test the methodology, an example from a published work, which was solved analytically, has been used for comparison purposes. The methodology to obtain coincident models from local models was tested mathematically with this example. Furthermore, the switching model of the simplified circuit of the interconnection of a source converter with a POL converter that behaves as a CPL was implemented, and the transfer functions were identified from this model. From this information, a linear analysis was performed and the proposed methodology was implemented to perform a nonlinear stability analysis.

The region of stability obtained using the identified models was practically coincident with the one obtained analytically. Besides, it was shown that the results obtained from the linear analysis cannot be extrapolated to define a ROA around a stable operating point. The resulting ROA was tested by checking the trajectories of the state variables, using the nonlinear equations, for different initial conditions. It was shown that the ROA actually has an upper bound, i.e. if the initial condition of the voltage is $v_{o}=350 \mathrm{~V}$ the system 
becomes unstable, which is not evident from the linear analysis because the linear systems around both $v_{o}=\{200,350\} V$ are stable.

The proposed methodology for large-signal stability analysis has been based on an example with a source converter connected to a CPL. In the future work, this methodology should be tested with more complex systems in order to validate its suitability for more general structures. Furthermore, the Lyapunov method is a sufficient condition, but not necessary. Therefore this method can result in conservative estimations, which depend on the Lyapunov candidate selected. In this work a quadratic structure is used, however in the future work other structures, as the ones shown in Section 2.4.2 should be considered, if possible, and the conservativeness of the results compared.

Even if the method would result only valid to study nonlinearities of the kind of the CPLs and the conclusions might be conservative, this method is considered of interest because of these two reasons: first, in dc microgrids the CPL behavior of the POL converters is one of the main source of instability in the system; second, to the best of the author's knowledge the proposed method is the first that attempts to perform a large-signal analysis using a blackbox approach, i.e. without the knowledge about the internal details of the converters, therefore it can provide a first estimation for system consisting of COTS converter, which otherwise would have to rely exclusively on small-signal methods.

This first approach towards a methodology to perform blackbox nonlinear stability analysis of EPC based systems is a contribution of this thesis. 


\section{Microgrid modeling}

$\mathrm{I}$

In Chapter 3 the different blackbox modeling structures were compared in different scenarios in order to identify their capabilities and their limitations. In Chapter 4 the integration of dynamic weighting functions within the polytopic model structure was proposed. It improves the accuracy of the model when the input variables change with large and sharp steps and the converter identified presented nonlinearities depending on the operating point. Finally, in Chapter 5 a new modeling approach was proposed in order to account for converters with highly variable dynamic behaviors, which is based on parameter varying transfer functions, where the parameters of the transfer functions are the ones modified through dynamic weighting functions.

According to the conclusions extracted from previous chapters, in Fig.7.1 the taxonomy of the suitability of the different blackbox modeling approaches for the EPCs according to their dynamic behavior is depicted. They are classified depending on their complexity from left to right. In general the more complex models are able to represent the converter with the characteristics located at their left side, however the simplest solution is to consider the most appropriate as they will be able to represent the dynamic behavior of the converter accurately with the lower computational burden.

In this chapter, the strategy to interconnect the individual blackbox models of the different EPCs will be detailed. Besides, some examples of dc microgrids with different control strategies will be presented. Finally, an experimental validation will be described.

\subsection{Interconnection of blackbox models}

The goal of this section is to provide a strategy to interconnect the blackbox models of the EPCs, which are identified independently. With this approach, the different models can be interconnected shaping any desired architecture. The Wiener-Hammerstein model (Fig. 2.10) can be readily used, as it consists of electrical elements. The method to interconnect two-port models, polytopic modes, or parameter varying transfer function models consists in setting the outputs of the model as inputs of controllable sources and measuring the inputs variables from the terminals of the system. A scheme of the interconnection strategy detailed is depicted in Fig. 7.2. It should be noted that other elements as line impedances or external filters can be included between the models in order to study their effect on the system performance or to improve it if necessary. 


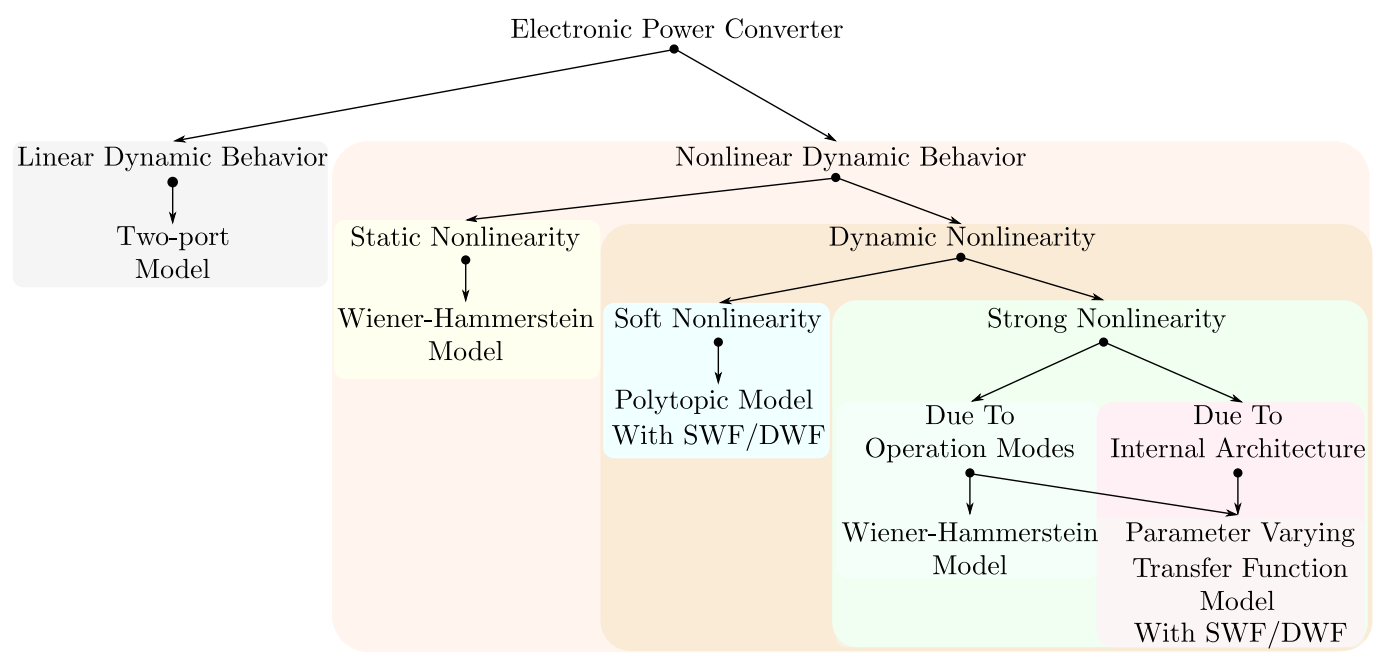

Figure 7.1: Taxonomy of the suitability of the different modeling approaches according to the dynamic behavior of the electronic power converters.

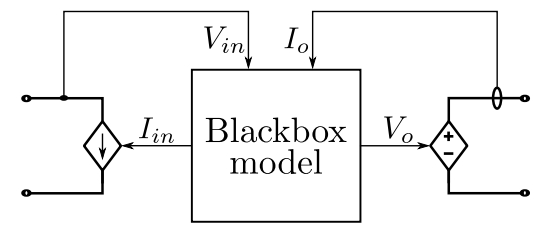

Figure 7.2: Scheme of the interconnection strategy among individual blackbox models.

In the following sections this interconnection strategy will be used to create the models of dc microgrids in different scenarios.

\subsection{DC microgrid with de bus signaling control}

\subsubsection{Inclusion of system-level control actions in the modeling structure}

In Section 2.1.2 it was described how an extended approach to deal with the parallel connection of sources controlling the bus voltage is to use droop controllers. This control strategy reduces the voltage reference of the converters according to their output current, therefore acting as a virtual output resistance. This technique allows a current sharing among the source converters, which can be related to their rated power. It is also possible to include upper level controllers that can act on the voltage reference in order to provide ancillary services like a controlled current sharing or voltage restoration to avoid the bus voltage deviation caused by the droop controllers.

In the Wiener-Hammerstein structure, this effect should be included in the static voltage reference. The proposed method to include the action of these control strategies into the blackbox two-port models is to include the voltage reference as a new input of the system. Therefore, two extra transfer functions must be introduced, in relation with the two outputs of the model (output voltage and input current in the case of the G-parameters model). In order to identify these two new transfer functions, a perturbation should be included in the voltage reference and the response of the two output variables measured, keeping the other two input variables constant. With this structure it is straightforward to include the effect of the droop control, where the droop parameter could be even variable, as represented in Fig. 7.3. 


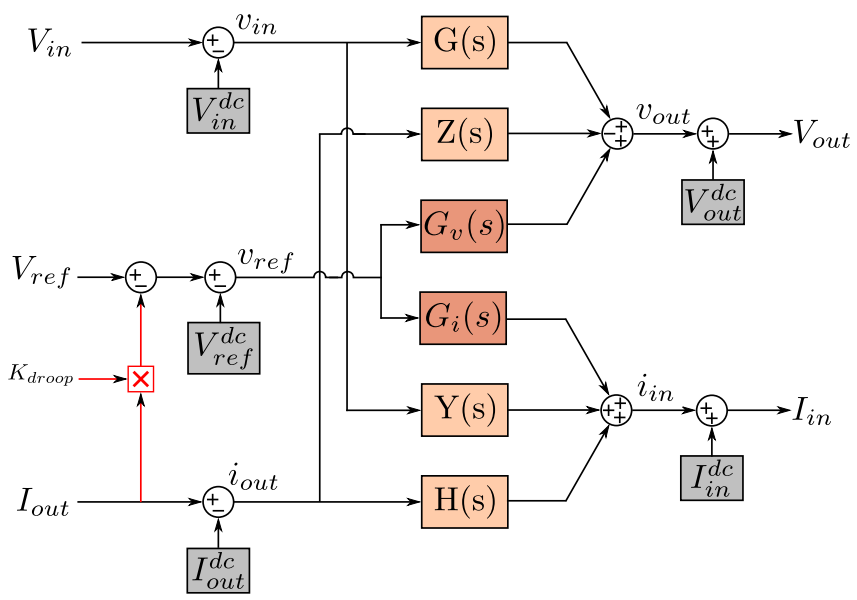

Figure 7.3: Scheme of the G-parameters model with the voltage reference as a new input and the inclusion of the droop control action.

In some cases, the dynamic due to the variation of the voltage reference is negligible compared with the dynamic related with the other input variables. In those cases, it is possible to simplify the model, avoiding the inclusion of the two extra transfer functions, by just considering the effect in the static part. The approach consists in modifying directly the operating point of the output voltage according to the droop control action:

$$
V_{\text {ref }}=V_{\text {out }}^{d c}-I_{\text {out }} K_{\text {droop }}
$$

where $V_{\text {ref }}$ is the new operating point for the output voltage. Furthermore, $K_{d r o o p}$ can be modified freely, allowing both testing and designing high level control strategies. Finally, as the dc value of the output voltage has been modified, the dc value of the input current should be adjusted accordingly to keep the power balance. Assuming the efficiency does not change, which is usually realistic considering the maximum output voltage variation is often limited to $5 \%$, the input current can be modified as follows:

$$
I_{\text {in }}^{2}=I_{\text {in }}^{1}\left(\frac{V_{\text {out }}-I_{\text {out }} \cdot K_{\text {droop }}}{V_{\text {out }}}\right)
$$

where the superscript 1 represents the operating point in which the model was identified and the subscript 2 represents the modified operating point taking into account the action of the droop controller.

\subsubsection{DC microgrid sources working with droop control and current limitation}

A dc microgrid switching model has been developed aiming to validate the application of blackbox models for its system-level design. A voltage droop control has been implemented in the sources that share the bus. The studied microgrid (Fig. 7.5) consists of a $24 \mathrm{~V}$ bus supplied by two isolated dc/dc converters: a full-bridge is connected to $380 \mathrm{~V}$, whereas a flyback is connected to $250 \mathrm{~V}$, which includes an inner current loop with a limited reference of $4 \mathrm{~A}$ and an outer output voltage loop with droop control. Two variable loads are supplied from the bus through regulated point-of-load converters. Finally, they are integrated by means of several line impedances. 


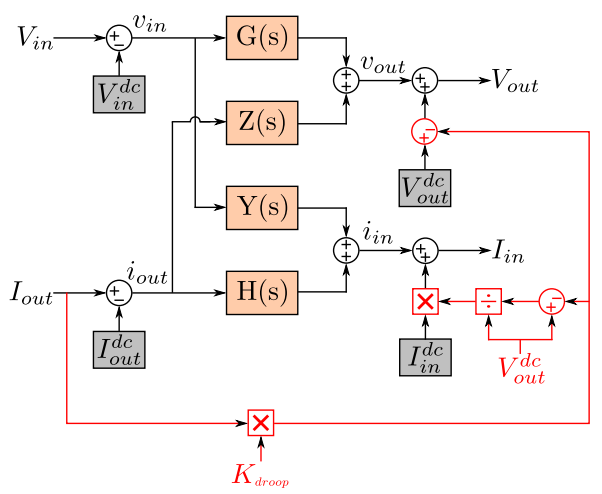

Figure 7.4: Scheme of the G-parameters model including the effect of the droop control in the static part of the model.

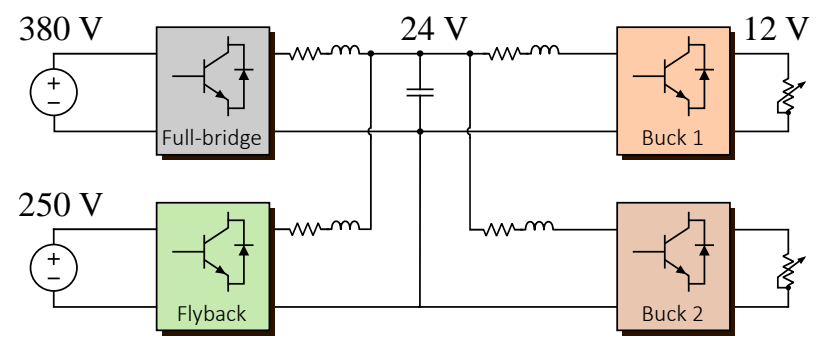

Figure 7.5: Studied dc nanogrid scheme.

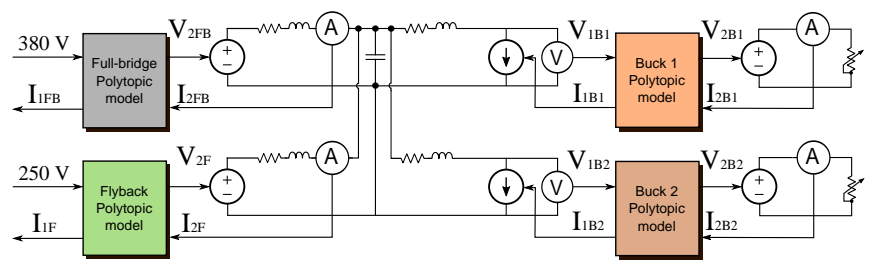

Figure 7.6: DC microgrid polytopic model scheme.

The proposed model takes advantage of the relation between voltage and current, established by the load impedance, to reduce the inputs of the model to those actually needed in real conditions, as detailed in Section 7.1. In Fig. 7.6 the scheme of the blackbox structure is presented, where only two inputs are necessary to simulate the microgrid.

First, the performance of the blackbox model of some of the converters is shown and compared with small-signal structures. The first comparison represents a slow load step to the POL converters, which are regulated buck converters. In order to capture the transition from DCM to CCM, which occurs around $I_{o}=4 \mathrm{~A}$ when $V_{i n}=24 \mathrm{~V}$, the output current is varied from $I_{o}=1.5 \mathrm{~A}$ to $I_{o}=5.5 \mathrm{~A}$, whereas the input voltage remains constant. In Fig. 7.7 the polytopic model, along with the linear G-parameters models implemented, is compared with the switching model. Small signal models around $I_{O}=3.5 \mathrm{~A}$ and $I_{o}=4.5 \mathrm{~A}$ have been included. The different linear models show relatively large differences in performance, e.g. the output voltage variation is 10 times higher in the case of the small signal model around $I_{O}=1 \mathrm{~A}$ than the model around $I_{O}=6 \mathrm{~A}$. This comparison shows how, with a slow load step, the polytopic model adjusts the weights of the different linear models according to the output current variation, resulting in a reasonably good approximation of the transition from DCM to CCM of the switching model.

Nevertheless, in dc distribution systems sharp load steps are not unusual. In case the 

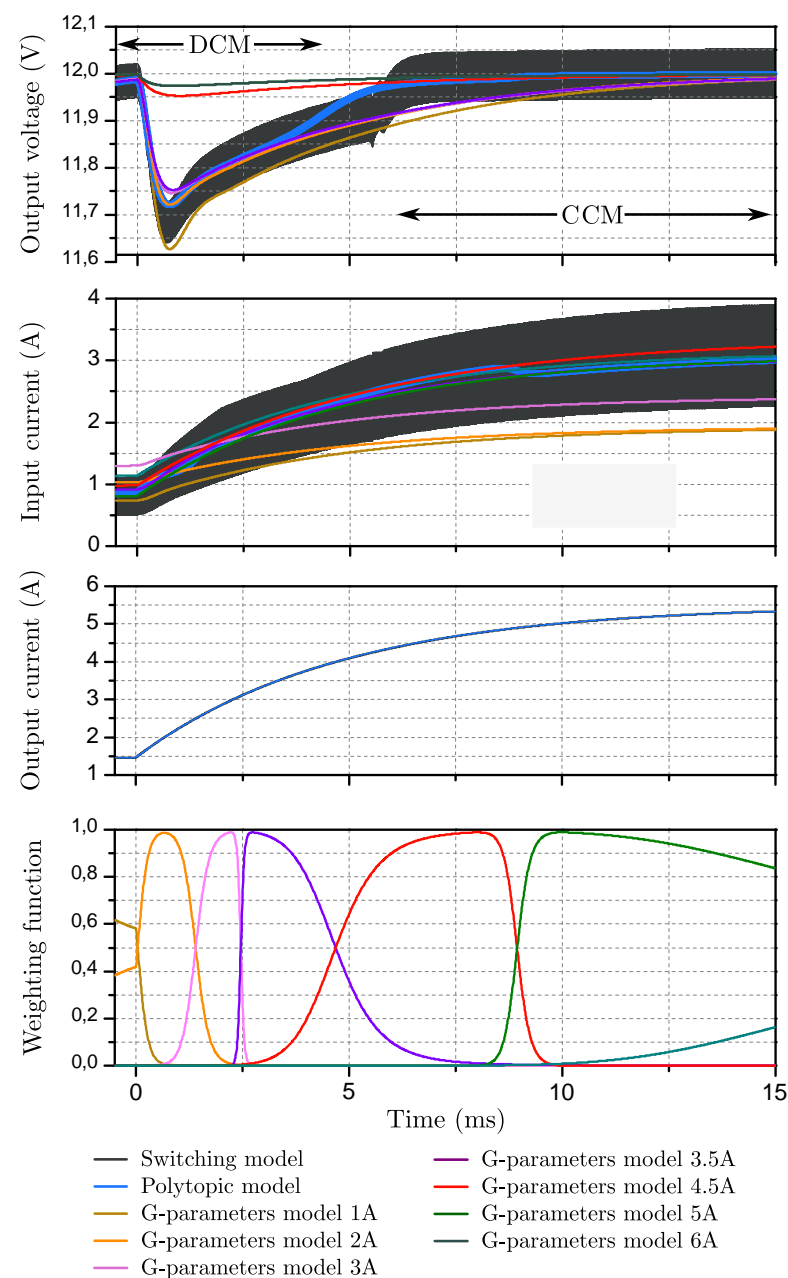

Figure 7.7: Polytopic model performance for a regulated synchronous buck converter with a slow step from DCM to CCM.

polytopic model uses the output current as an input parameter, the sudden load step will cause an abrupt change between the linear models involved, as studied in Section 3.1.1. In Fig. 7.8 this situation is represented. In this case, the steady state is still well represented, whereas noticeable differences are found in the transient behaviors. This can be explained by the dynamic of the regulator, which is ignored by the polytopic model. In order to overcome this problem, the polytopic model with DWF is implemented, as studied in Section 4.3. The improvement in the transient behavior is also depicted in Fig. 7.8, where the light blue signal represents the polytopic model using the DWF. Also the filtered signals which are used as inputs for the scheduling functions are depicted in gray.

As for changes in the power converter control mode, the linear model of the converter working in constant current mode must be obtained and, instead of using weighting functions for the transition, perform an abrupt change to this linear model when the proper conditions are met, as detailed in Section 3.2.

In order to test these modeling approaches, a full-bridge switching model has been designed using a double control loop, the inner is a fast current control, whereas the outer is a slow voltage control loop. The change of control mode is studied by means of a current limitation, imposing a 6 A reference to the inner loop when the outer loop sets a reference higher than $6.2 \mathrm{~A}$. 


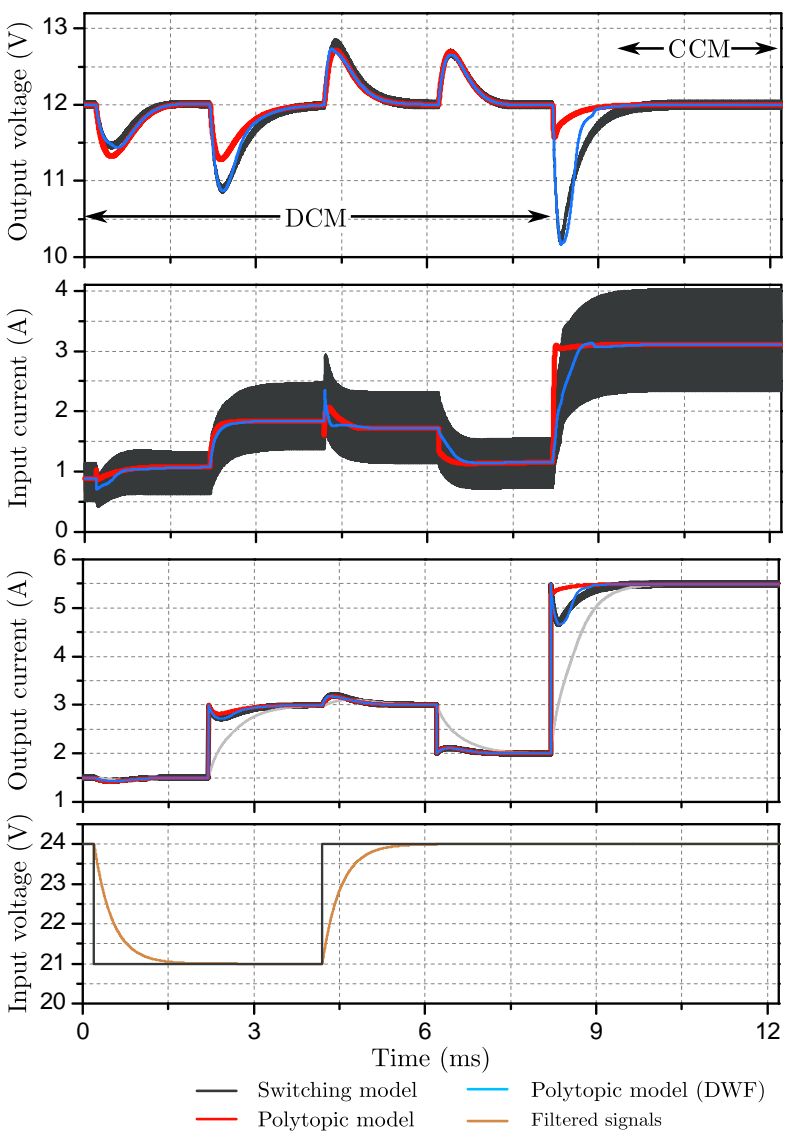

Figure 7.8: Polytopic model performance for a synchronous buck converter with sharp steps.

The condition used in the polytopic model for switching to the current limited linear model is the same, output current higher than $6.2 \mathrm{~A}$, however to go back to the voltage controlled models two conditions are included: the output current must be less than $6 \mathrm{~A}$ and the output voltage of the current limited model must be higher than the output voltage of the voltage controlled models. The latter considers the transient when the output voltage recovers its controlled value. Indeed, while the output voltage is lower than the reference, the output current of the voltage controlled linear models will be higher than 6.2 A and an oscillation between current limited and voltage controlled models would arise without the mentioned condition. Finally, notice that the switching converter measures the inductor current, while the polytopic model uses the output current, hence in this case it is particularly important to use the dynamic limitation imposed by the DWF for the input of the scheduling functions.

The performance of the black-box model under these phenomena is presented in Fig. 7.9. The droop control behavior is represented with a sharp load step, where the output current changes from $I_{o}=1.5 \mathrm{~A}$ to $I_{o}=5.5 \mathrm{~A}$, whereas the change in the control mode occurs when a second load step attempts to rise the output current beyond 6 A. Notice how the delay in the transition from voltage to current control has been well described owing to the dynamic limitation, which is also depicted in gray along with the output current. Finally, both the transient and the steady state after the change of control of the black-box model are in good agreement with the switching model. One of the G-parameters models has been included to compare changes in transient and steady state behavior from small-signal to polytopic model. 

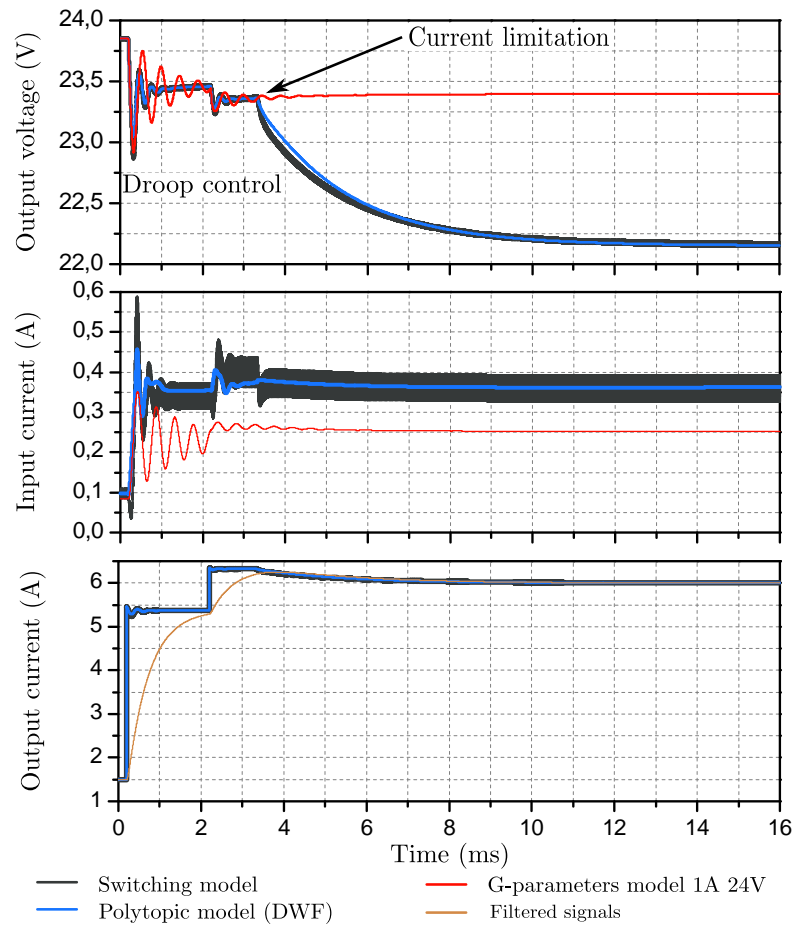

Figure 7.9: Polytopic model performance for a full-bridge converter using droop control and current limitation.

The blackbox models have been developed according to the methodology previously detailed. Afterwards, these models were integrated in the microgrid and both responses were compared under different conditions. In Fig. 7.10 the comparison between switching model and blackbox model signals is presented.

The results demonstrate the capability of the blackbox polytopic models with DWF to reproduce the dynamic behavior of the converters. Additionally, the change of the control mode in the flyback, from voltage control to current control, is well reproduced. Furthermore, a load disconnection that makes the flyback converter work with droop control again has been included. Notice how this transition causes a peculiar phenomenon in the output current of the flyback model due to the bus voltage recovery, which is in good agreement in both models. Finally, the interactions between converters are also accurately reflected by this structure.

\subsubsection{DC microgrid sources working with droop control, MPPT control, and system-level control strategies}

In this section a microgrid consisting of sources controlling the bus voltage in parallel through droop controllers is presented. The converter interfacing a renewable source should work with a MPPT control, unless the microgrid is not able to absorb all the energy available. In that case the converter will change to droop control to provide only the necessary power and avoid overloading the microgrid. Finally, some system-level control strategies are included to control the current sharing among the source converters and to restore the bus voltage.

Figure 7.11 depicts the microgrid scheme composed of three source converters that feed a $24 \mathrm{~V}$ bus: one connected to a renewable source, one connected to the grid or a higher level bus, and one connected to a battery. In addition, some POL converters are connected 

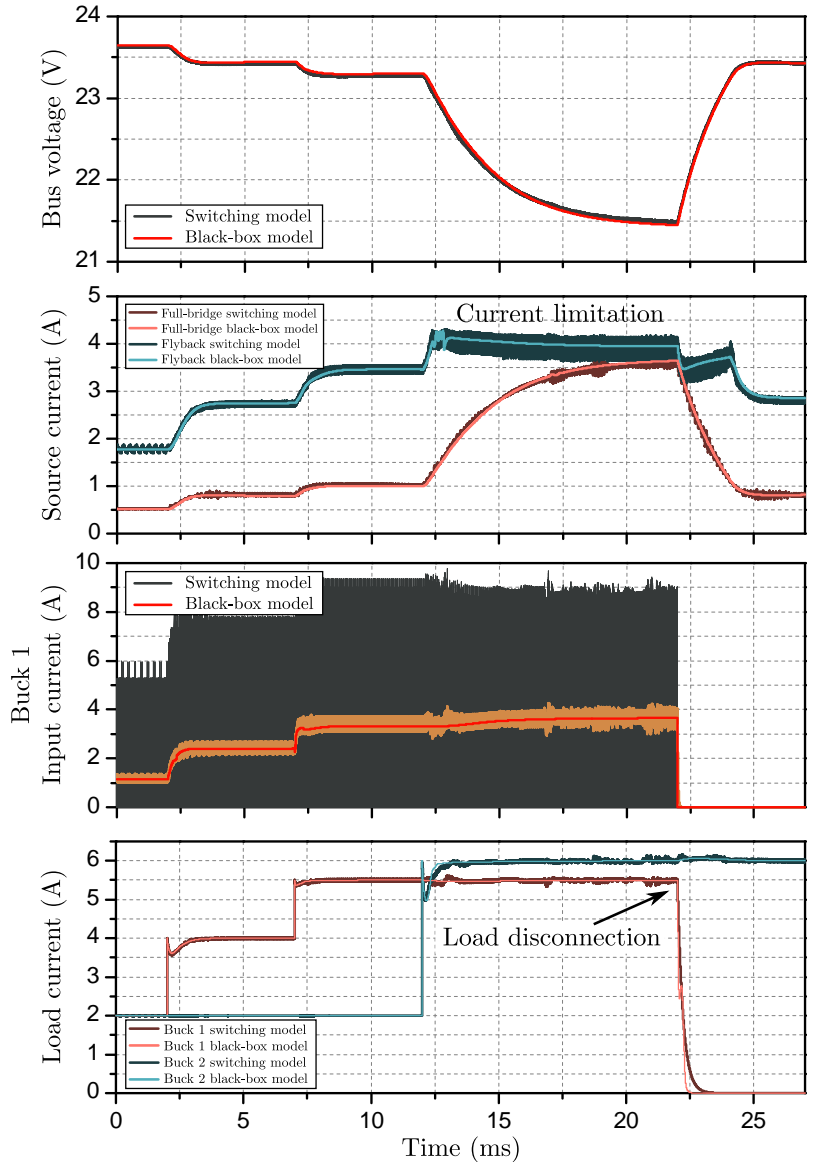

Figure 7.10: Polytopic model performance for a dc microgrid with droop control, current limitation and load disconnection.

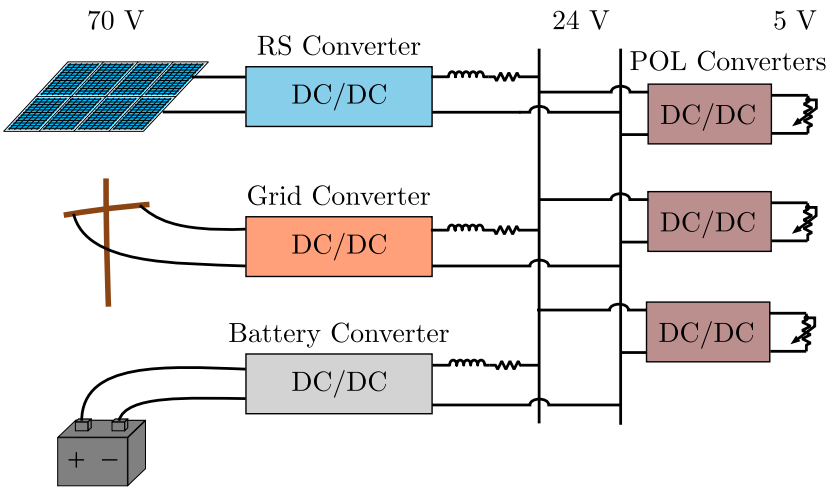

Figure 7.11: Microgrid scheme.

to the low voltage bus demanding variable power.

First, the blackbox models of the EPCs will be described.

\section{Renewable source converter}

The converters interfacing RS should use MPPT control in order to utilize all the energy available. However, in case the microgrid is not able to absorb this amount of power, they 


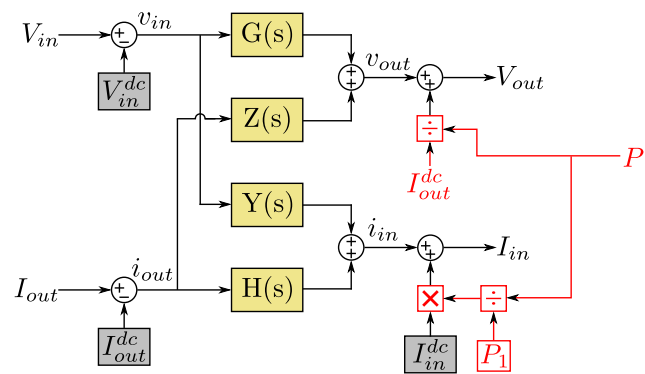

Figure 7.12: Small-signal model including the MPPT control effect to consider a variable power delivered by the RS.

must change their control strategy to deliver just the power that is needed. Therefore, in those cases the converter will work regulating the bus voltage by means of the droop control technique (see Fig. 2.4).

In order to model a converter with different control strategies, it is necessary to obtain a model for each of them. In general a polytopic model of the converter working with each control strategy will be obtained, which will consider as many small-signal models as needed to model the behavior of the converter around the operating points of interest.

Modeling a converter with droop control was considered in [120]. G-parameters were used as the two-port small-signal models so the input voltage and output current were used as variables. Strictly speaking, the droop parameter should be another input variable, however, in order to reduce the number of signals taken into account, its effect was approximated by modifying the dc value of the output voltage according to the droop equation (see Fig. 7.4).

Similarly, G-parameters can be used for the converter with MPPT control and the behavior of different power levels can be approximated by defining the dc output voltage as a function of the RS power:

$$
V_{\text {out } D C}=\frac{P}{I_{D C}}
$$

Accordingly, the input current, the other output variable, must be adjusted. Assuming the efficiency does not change considerably, the new input current could be modified as follows:

$$
I_{i n D C 2}=I_{i n D C 1} \cdot \frac{P_{2}}{P_{1}}
$$

where $I_{i n D C 2}$ is the input current with the new power level, $P_{2}$, and $I_{i n D C 1}$ is the input current with the power level at which the model was obtained, $P_{1}$, (Fig. 7.12).

The transition between these two control techniques can be performed with weighting functions or by switching according to suitable conditions. The former is usually more appropriate considering the transition nature. The operating point around which the switch occurs, $p_{1}$ of Fig. 7.13, will be determined by the intersection of the droop line and the constant power hyperbola in the V-I graph:

$$
V_{D C}-K_{\text {droop }} \cdot I_{\text {cross }}=\frac{P}{I_{\text {cross }}}
$$




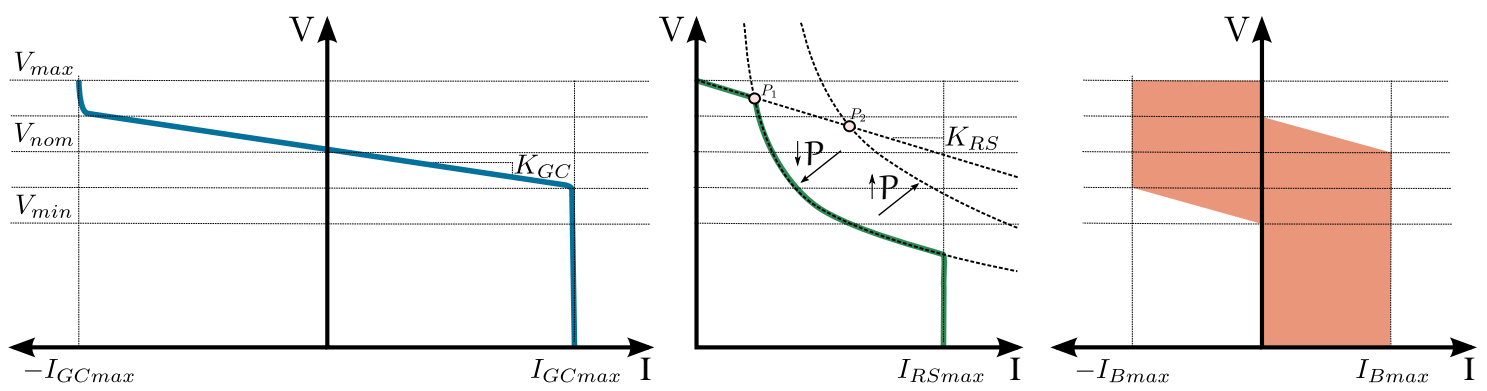

Figure 7.13: DC bus signaling strategy scheme. (a) Grid converter, (b) Renewable source converter, (c) Battery converter.

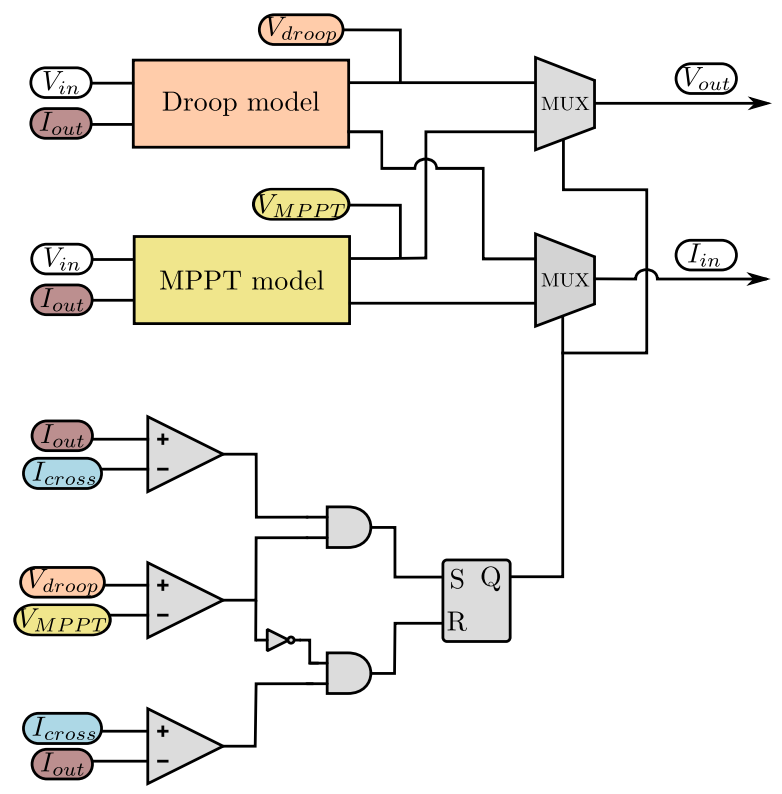

Figure 7.14: Implemented conditions to switch from the response of droop to MPPT controlled polytopic models.

$$
I_{\text {cross }}=\frac{\frac{V_{d c}}{K_{\text {droop }}}-\sqrt{\left(\frac{V_{d c}}{K_{d r o o p}}\right)^{2}-4 \cdot \frac{P}{K_{d r o o p}}}}{2}
$$

where $V_{D C}$ is the nominal voltage of the bus, $K_{\text {droop }}$ is the droop parameter, and $P$ is the power delivered by the RS. Besides, in the moment of the switch, the output voltage of the constant power model must be lower than the droop model to change from droop to MPPT and the opposite in the other direction. These conditions are depicted in Fig. 7.14.

A buck converter using the previous detailed control technique has been designed in order to test the proposed modeling strategies, using $V_{d c}=24 \mathrm{~V}, K_{\text {droop }}=0.15$ and $P=$ $150 \mathrm{~W}$. Two events are of special interest in this case, the transition from droop control to MPPT control and power changes.

Fig. 7.15 focuses on the first situation. Three load steps are performed: after the first the converter still works using voltage droop control, the second causes the transition from droop to MPPT, and with the third it remains using MPPT. At the expense of clearness, in this figure some of the small-signal responses are depicted as well to illustrate the nonlinear 

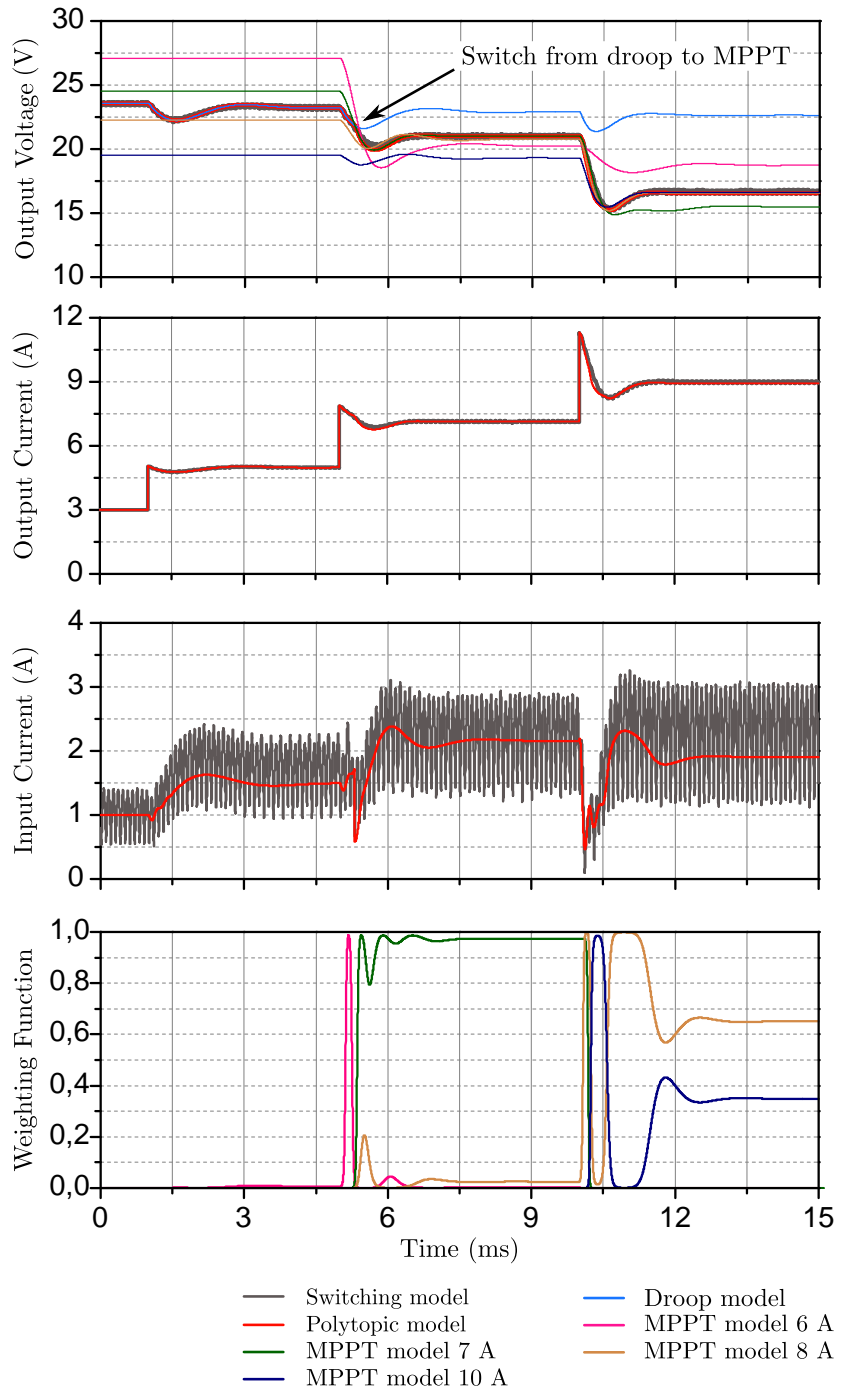

Figure 7.15: Performance of the proposed polytopic model for a buck converter using droop-MPPT control strategies.

behavior of the converter. It can be seen how the polytopic model smoothly changes among the different small-signal responses to accurately represent the converter behavior in this situation.

The second event is shown in Fig. 7.16. This time two different experiments are represented together, only changing the maximum power level from $P_{1}=150 \mathrm{~W}$, to $P_{2}=200 \mathrm{~W}$. The same two load steps are performed in both tests. After the first, in the case of $P=150 \mathrm{~W}$ the converter enters in MPPT control mode, whereas in the case of $P=200 \mathrm{~W}$ the converter stays using droop control. Finally, with the second load step the converter with $P=200 \mathrm{~W}$ also moves to MPPT control. The proposed model is shown to be in good agreement with the switching model behavior, representing the dependence of the transition from droop to MPPT with the power delivered by the RS, and also the steady-state and dynamic behavior with precision. 

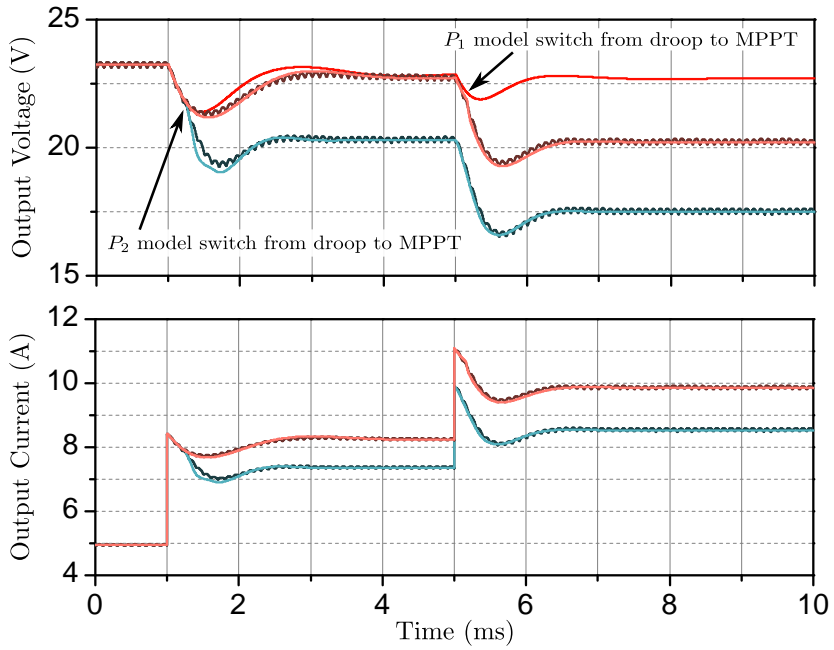

$\begin{array}{lll}\text { Switching model for } P_{1} & - \text { Switching model for } P_{2} \\ \text { Polytopic model for } P_{1} & - \text { Polytopic model for } P_{2} \\ \text { Droop model } & \end{array}$

Figure 7.16: Performance of the proposed simplified model to take into account changes in the maximum power from $P_{1}=150 \mathrm{~W}$ to $P_{2}=200 \mathrm{~W}$.

(a)

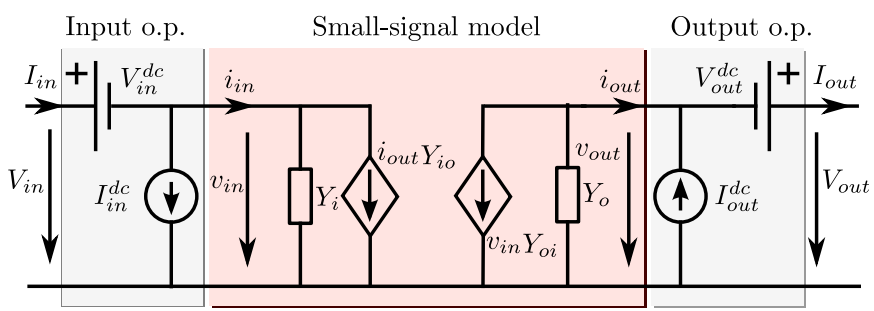

(b)

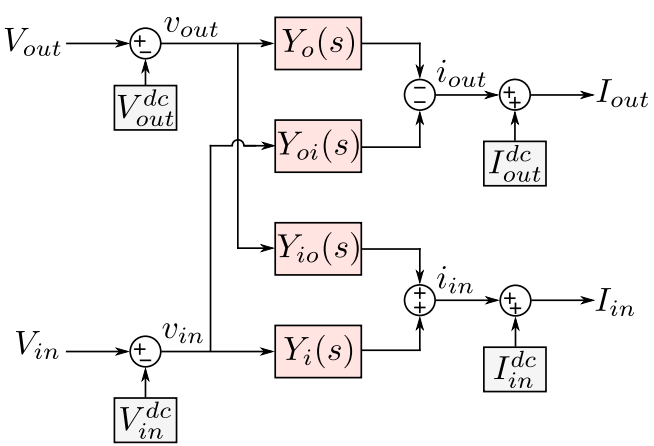

Figure 7.17: Admittance (Y) parameters scheme. (a) Equivalent circuit, (b) block diagram representation.

\section{Battery converter}

The converters interfacing batteries will, in general, work with constant current or droop control. The more suitable two-port model for current controlled converters is the Yparameters (admittance parameters). It considers a Norton equivalent model both for the input and the output of the converter (Fig. 7.17).

In case the output current is variable, the dynamic behavior of the converter under changes in the current reference should be included. In order to do so, two new transfer 
functions have to be included:

$$
\left(\begin{array}{c}
i_{o} \\
i_{i}
\end{array}\right)=\left(\begin{array}{ccc}
Y_{o}(s) & Y_{o i}(s) & H_{o r e f}(s) \\
Y_{i o}(s) & Y_{i}(s) & H_{\text {iref }}(s)
\end{array}\right)\left(\begin{array}{c}
v_{o} \\
v_{i} \\
i_{\text {ref }}
\end{array}\right)
$$

where $H_{\text {oref }}(s)$ and $H_{\text {iref }}(s)$ can be obtained analyzing the output and input current small-signal response with current reference perturbations, when both the input and output voltages are kept constant.

In the following these models will be used for system-level modeling. Also the integration of secondary-level control techniques into the polytopic model of the different converters will be discussed.

\section{Microgrid simulation}

This microgrid has been designed using buck converters working with the control strategies previously detailed. Following the methodology described in Chapter 4, a polytopic model of this microgrid has been obtained using a blackbox approach. The comparison can be seen in Fig. 7.18. In this simulation both grid and RS converters have a $K_{\text {droop }}=0.15$. The solar panel is providing a power $P=150 \mathrm{~W}$ and the battery is recharging using constant current.

First, the RS converter is working in parallel with the grid converter using droop control. Despite having the same droop parameter, $K_{\text {droop }}$, their output current is different because they have different line resistances. At $t=5 \mathrm{~ms}$ there is a load step and the sources share the increase of current according to the droop control strategy. However, at $t=15 \mathrm{~ms}$ two simultaneous load steps cause that the RS converter switches to MPPT control. After, at $t=20 \mathrm{~ms}$ the battery increases the demanded current, when the RS converter is providing maximum power and the bus voltage is controlled by the grid converter. Finally, at $t=30 \mathrm{~ms}$ there is another load step and an increase in the current demanded by the battery. It can be seen how the extra current is provided by the grid converter and the RS converter output current is adapted according to the deviation of the bus voltage, in order to keep its constant power. The polytopic models represent nicely all these effects.

Following, the inclusion of the secondary level techniques in the polytopic model is studied.

\section{Voltage restoration}

A possible disadvantage of the droop control is the voltage deviation from the nominal value, which is used to share the current among the converters controlling the bus voltage in parallel. In case this deviation is troublesome, it can be restored using low-bandwidth frequency communication. The idea is to measure the voltage deviation of the bus and add this value, using a PI regulator, to all the voltage references of the converters working with droop control.

$$
\begin{aligned}
\delta v & =K_{p}\left(V_{D C}-V_{b u s}\right)+K_{i} \int\left(V_{D C}-V_{b u s}\right) \\
V_{\text {refi }} & =V_{D C}-K_{d r o o p i} \cdot I+\delta v
\end{aligned}
$$

where $\delta v$ is the bus voltage deviation, $K_{p}$ and $K_{i}$ are the proportional and integral gains of the PI regulator, $V_{D C}$ is the nominal voltage of the bus, $V_{b u s}$ is the bus voltage, and $V_{\text {refi }}$, 

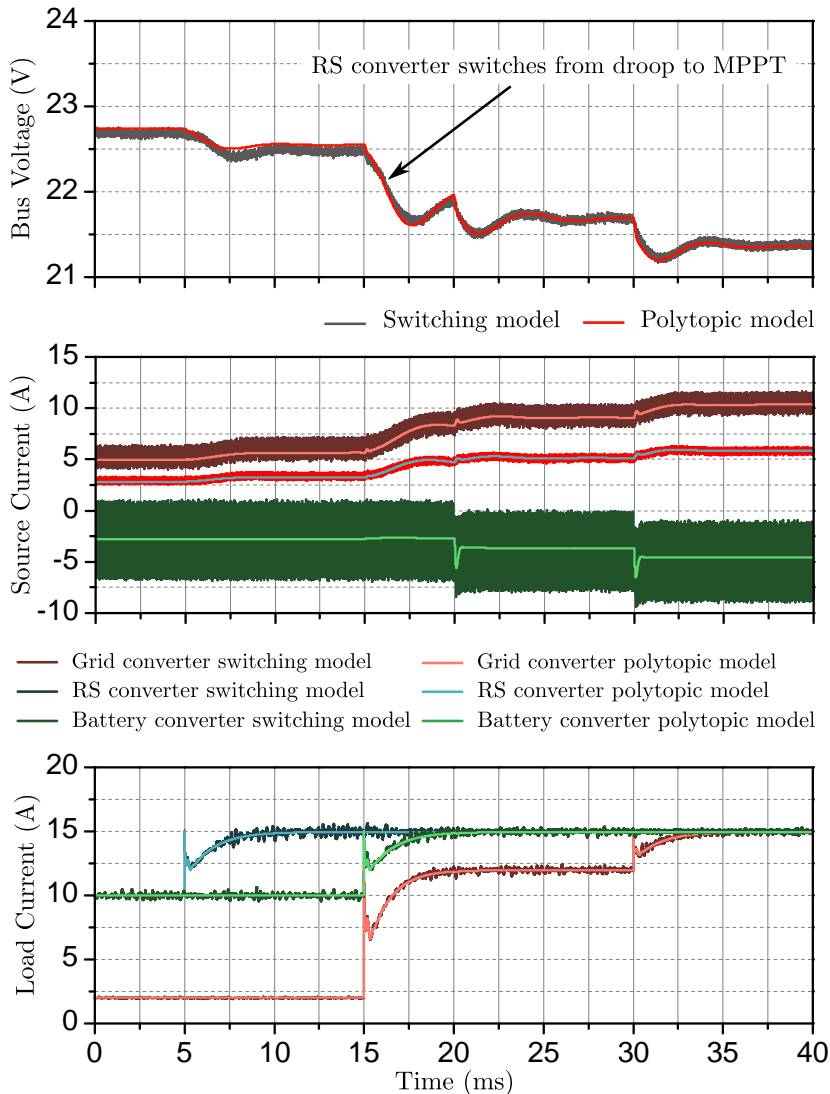

$\begin{array}{lll}\text { — POL converter } 1 \text { switching model } & \text { POL converter } 1 \text { polytopic model } \\ \text { — POL converter } 2 \text { switching model } & \text { POL converter } 2 \text { polytopic model }\end{array}$

Figure 7.18: Performance of the proposed polytopic model for a system-level simulation with changes in the operation mode of the converters.

$K_{d r o o p i}$ are the voltage reference and the droop parameter of each of the converters using droop control.

In order to include this technique into the polytopic model, the dc output voltage of the small-signal models can be defined as the voltage reference. In case this approximation does not provide accurate enough results, a new transfer function should be included to consider the dynamic behavior of the converter under a change in the voltage reference, as shown in Fig. 7.3. The input-output equations of the converter would be as follows:

$$
\left(\begin{array}{c}
v_{o} \\
i_{i}
\end{array}\right)=\left(\begin{array}{ccc}
G(s) & -Z(s) & G_{r e f}(s) \\
Y(s) & H(s) & Y_{\text {ref }}(s)
\end{array}\right)\left(\begin{array}{c}
v_{i} \\
i_{o} \\
v_{r e f}
\end{array}\right)
$$

This technique has been applied to the previous test and the results are shown in Fig. 7.19. It can be seen how the output voltage of the converters controlling the bus voltage is increased to restore the bus voltage after each perturbation. Consequently, the output current of the sources is lowered. The comparison shows how these effects and the dynamic behavior is well represented by the polytopic models. 

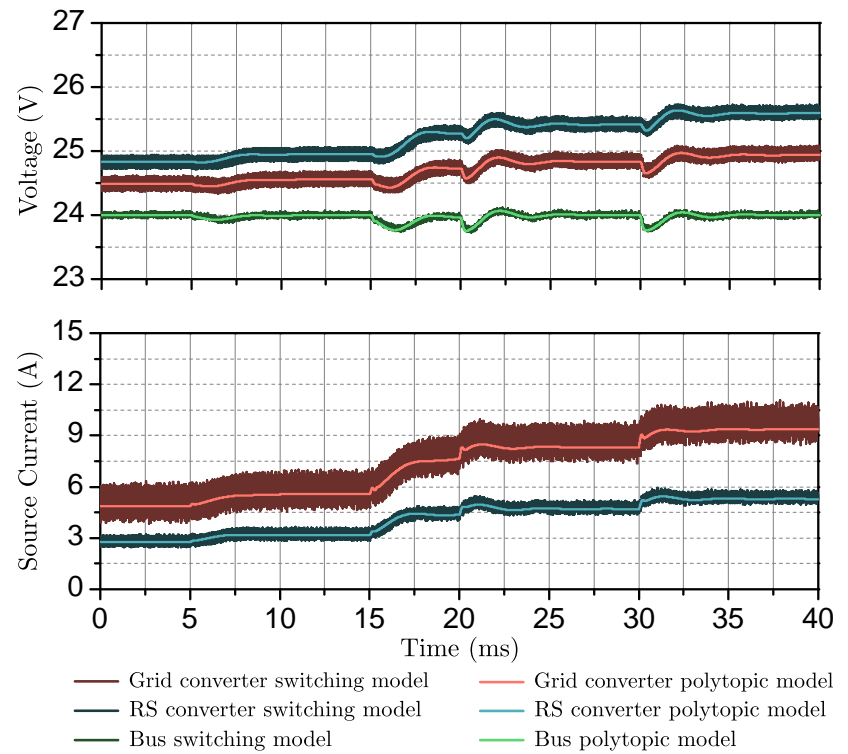

Figure 7.19: Performance of the proposed polytopic model to simulate the effect of the voltage restoration control technique.

\section{Current sharing}

The other possible drawback is the effect of the line impedances on the current sharing among the converters regulating the bus in parallel. As these impedances are not taken into account, they can unbalance the current delivered by each converter.

Again, by using low-bandwidth frequency communication it is also possible to deal with this problem. A possible solution is to measure the output current of each converter, obtain the arithmetic average value and modify the voltage reference of the converters with the error by means of a PI regulator.

$$
\begin{aligned}
i_{\text {avg }} & =\frac{\sum_{j=1}^{n} i_{j}}{n} \\
\delta i_{i} & =K_{p}\left(i_{\text {avg }}-i_{i}\right)+K_{i} \int\left(i_{\text {avg }}-i_{i}\right) \\
V_{\text {refi }} & =V_{D C}-K_{\text {droopi }} \cdot I+\delta i_{i}
\end{aligned}
$$

where $i_{\text {avg }}$ is the average value of the output currents of the converter, $i_{j}$ is the output current of each converter, $n$ is the total number of converters that should have the same output current, and $\delta i_{i}$ is the output current error of each converter. The incorporation of this strategy in the polytopic model can be performed in a similar manner as explained before for voltage restoration.

The previous simulation is repeated, but this time, at $t=10 \mathrm{~ms}$, the current sharing control is activated. The comparison is depicted in Fig. 7.20. It can be seen how the converter providing more current decreases its output voltage and vice-versa until their output currents are equalized. However, at $t=20 \mathrm{~ms}$ the RS converter switches to MPPT control and the controls are not able to get both objectives due to the maximum power limitation.

These simulations show how the proposed modifications to the polytopic model can be useful to study the effect of system-level control strategies from a blackbox perspec- 

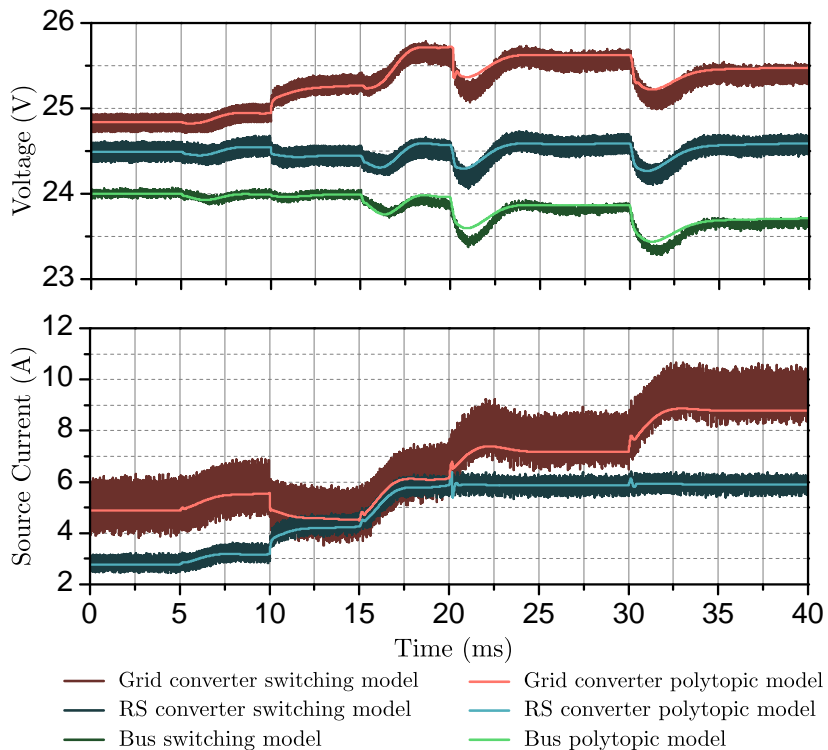

Figure 7.20: Performance of the polytopic model to simulate the current sharing control technique.

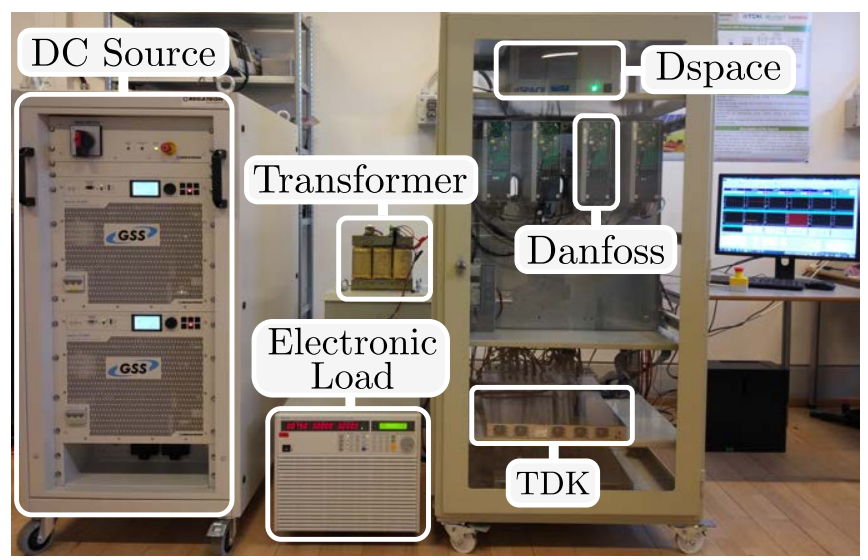

Figure 7.21: Experimental setup implemented.

tive. Therefore, commercial converters can be tested individually in order to obtain their polytopic models and, afterwards, the techniques proposed can be added to the model in order to be able to simulate how they would behave together in a microgrid. Also, the secondary control could be adjusted before the real microgrid has been assembled, avoiding unexpected failures.

\subsection{Experimental validation: microgrid with droop control and system-level control strategies}

An experimental setup (see Fig. 7.21) has been assembled in order to validate the blackbox modeling techniques described above to predict the dynamic behavior of dc microgrids. In this section it will be described how the system has been arranged and how it has been identified. 


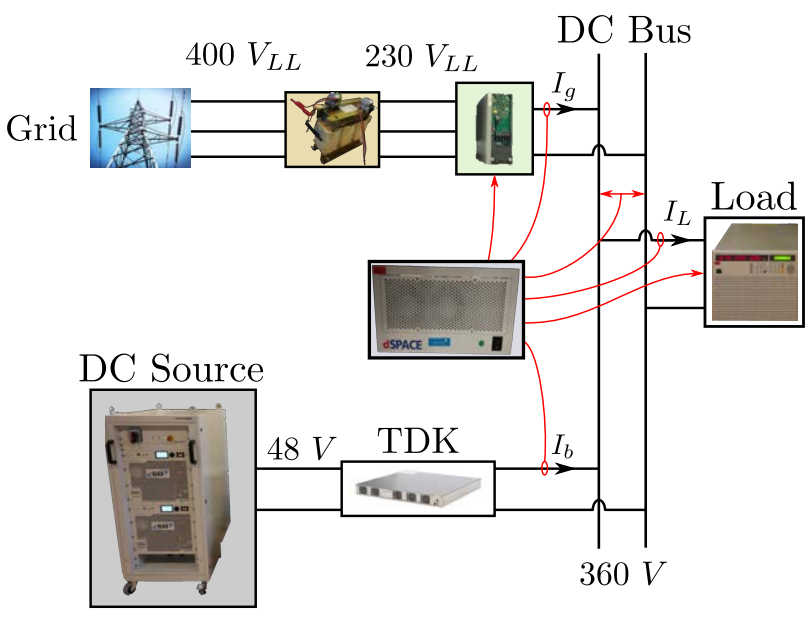

Figure 7.22: Scheme of the experimental setup.

\section{System Description}

The system consists basically of two sources controlling in parallel the bus voltage and an electronic load. It contains the minimum number of elements that allow checking the phenomena of interest, i.e. parallel and series connection of power converters, parallel connection of voltage source converters, secondary-level control strategies and load variations.

One of the sources is a rectifier connected to the electric grid, which will represent the generation units of the ship. A stepdown transformer was introduced to reduce the line to line voltage below the desired bus voltage and to provide galvanic isolation. The bus voltage was set to $360 \mathrm{~V}$ in order to keep it safely within the COTS converter rating values. The other source is connected to a $48 V_{d c}$ power source, which represents a storage unit. These source converters and their blackbox models will be further detailed below. Finally, a Chroma dc electronic load (model 63204) feeds from the dc bus, working as a controlled current load. A hardware-in-the-loop device (Dspace version DS1006) has been used to perform the control of the rectifier, to set the waveform of the current demanded by the electronic load and to capture the currents and voltages of interest. Fig. 7.22 depicts the scheme of the system described.

\section{Rectifier Model}

The rectifier converter has been implemented with a Danfoss FC-302 IP20 $2.2 \mathrm{~kW}$ [121]. This converter has a three-phase IGBT bridge and an EMC filter in the dc side. In the ac side a LCL filter was externally added. The control has been integrated by means of the Dspace HiL device. The control consists of a PLL to synchronize with the grid frequency and an inner input current control and an outer output voltage control to regulate the bus voltage. The inner controller is the one proposed in [122], where a detailed description of the control and its design can be found. The voltage control includes droop control and the possibility to add a secondary control for current sharing or voltage restoration purposes. Fig. 7.23 depicts the structure described above.

The focus of this work is on the dc part of the rectifier, so no perturbations are considered in the grid voltage. Under this assumption, only the output impedance from the G-parameters (2.8) is needed to characterize the behavior of the output voltage. Furthermore, the transfer function from the control to the output voltage was identified, in order to be able to account for the effect of secondary controllers.

The output impedance of the rectifier was identified with the grid as a source and the 


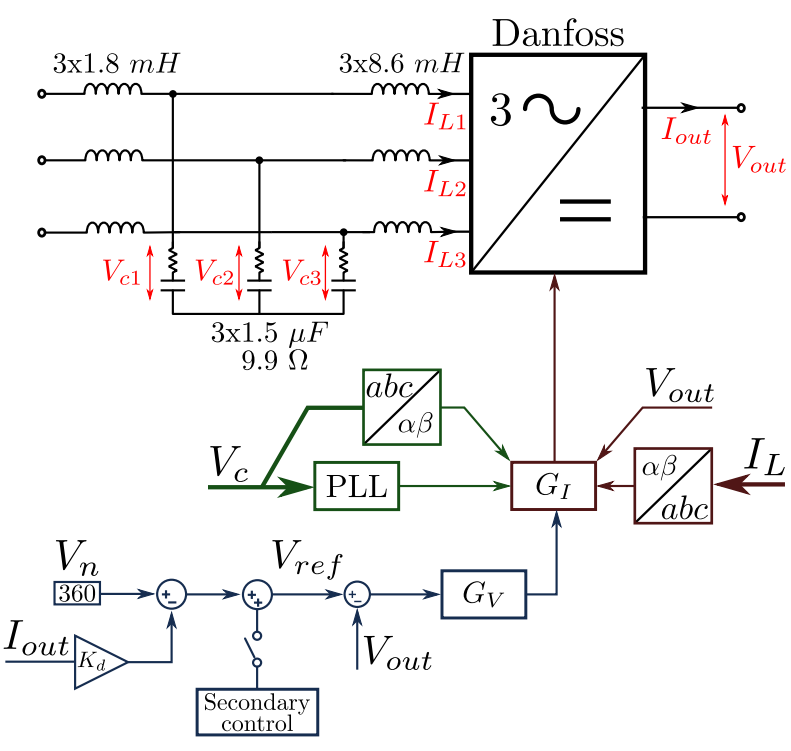

Figure 7.23: Scheme of the rectifier and its control structure.

electronic load as the load. The voltage reference was set to $360 \mathrm{~V}$, the droop parameter was set to 0 and no secondary controls were included. The identification was performed in time domain, as the electronic load used is able to provide enough slew rate for the load steps. Notice that in time domain, a high slew rate is necessary to excite high frequencies:

$$
\text { slew rate } \geq 2 \pi f A_{p k}
$$

where $f$ is the frequency of a sinusoidal signal with a peak value of $A_{p k}$. The maximum slew rate of the electronic load is $25 \mathrm{~A} / \mu \mathrm{s}$. Assuming that a $0.5 A_{p k}$ sinusoidal is well above the noise level, the maximum observable frequency is around $8 \mathrm{MHz}$, which is much higher than the switching frequency of the converter $(10 \mathrm{KHz})$. With this configuration, the electronic load in current mode was set to different current values and steps of $10 \%$ of these values were performed to obtain the dynamic behavior of the converter. This value was selected as a good compromise between high enough to avoid the effect of the noise and small enough to ensure a small-signal behavior.

From these tests it was observed that the converter has a different dynamic behavior at light load, $I_{\text {out }}$ from 0 to $0.5 A(0-180 \mathrm{~W})$, compared to the behavior at higher values of the load. For values of the output current above $1 A$, it was observed that the dynamic behavior did not vary considerably. The data of the response of the output variables to the input perturbations was used in the System Identification toolbox of Matlab in order to obtain the transfer functions. According to the observed behavior of the converter, two transfer functions were obtained: one at $I_{\text {out }}=0.1 \mathrm{~A}$ and another at $I_{\text {out }}=1 \mathrm{~A}$. Subsequently, a polytopic model with dynamic weighting functions was designed selecting the poles of the transfer functions that better approximate the transition between smallsignal models. Similarly, the $G_{c}$ transfer functions, described in (2.12), were obtained at the two operating points mentioned before. Finally, the blackbox model was assembled as sketched in Fig. 7.24 and the transfer functions obtained are presented in Table 7.1. The static weighting functions used are the classical double sigmoids: 


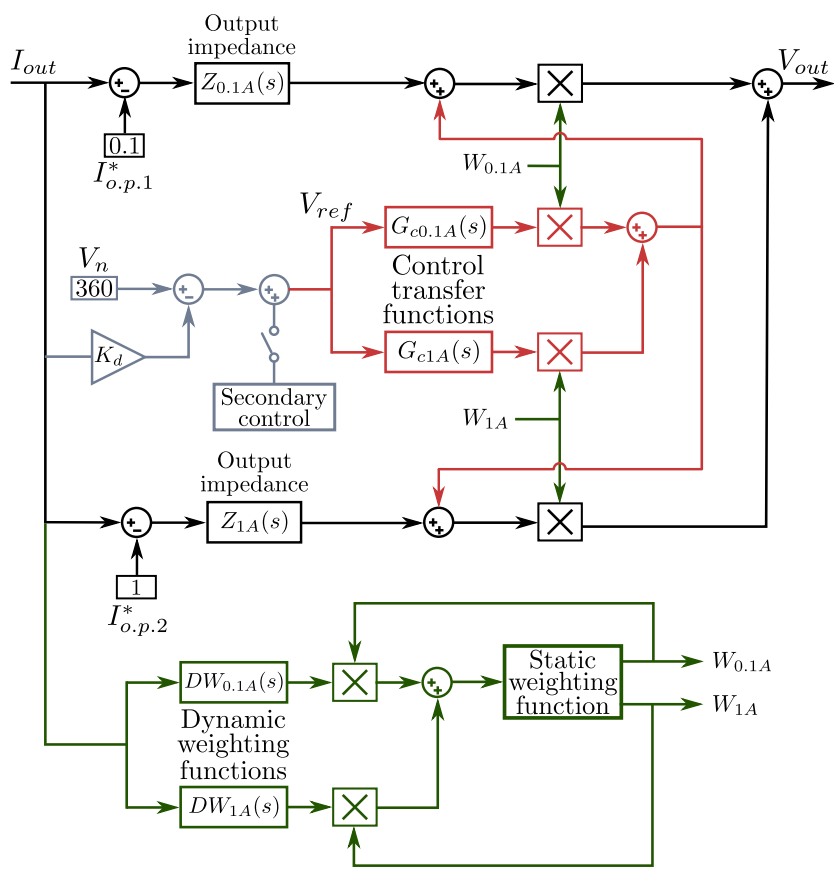

Figure 7.24: Sketch of the blackbox model of the rectifier.

$$
\begin{aligned}
\omega_{i}\left(I_{\text {out }}\right)= & \left(\frac{1}{1+e^{-m_{i}\left(I_{\text {out }}-c_{i}\right)}}\right)- \\
& \left(\frac{1}{1+e^{-m_{i+1}\left(I_{\text {out }}-c_{i+1}\right)}}\right)
\end{aligned}
$$

where $\omega_{i}\left(I_{\text {out }}\right)$ are the weighting functions of the small-signal models $i$, which depend on the output current, $I_{\text {out }}, m_{i}$ and $c_{i}$ are the slope and the center of the rising edge of the sigmoid and $m_{i+1}$ and $c_{i+1}$ are the slope and the center of the falling edge. In this case, the two models have one interface interval with a slope $m=20$ and a center in $c=0.4 \mathrm{~A}$. These parameters were tuned with the response of the system to a step from one operating point to the other. As mentioned earlier, the identification process of the polytopic models with dynamic weighting functions has been defined in such a way that algorithms can be applied for the automation of the model obtainment. From the response of the system to small and large-signal steps perturbations in the input variables, the program should be able to select the number of operating points to be considered, the slope and center of the weighting functions, and the poles to be included in the transfer functions of the dynamic weighting functions. These decisions are subjected to a trade-of between accuracy and complexity of the overall model.

\section{DC-DC Converter Model}

The dc-dc converter has been implemented with a TDK-Lambda EZA $25002.5 \mathrm{~kW}$ bidirectional dc/dc converter 320/48 V (300-380 / 36-60 V) [123]. This converter was used with its own controller and no external components were added. The control includes droop and it can be modified by means of serial communication RS-485. In order to make the identification it was connected to the dc source, a Regatron TopCon TC.GSS, and the electronic load. Once more, the load was set to current mode and $10 \%$ steps were performed at different current values. No substantial changes in the dynamic response were observed with the load variation; therefore a small-signal model was enough to represent the converter in the 


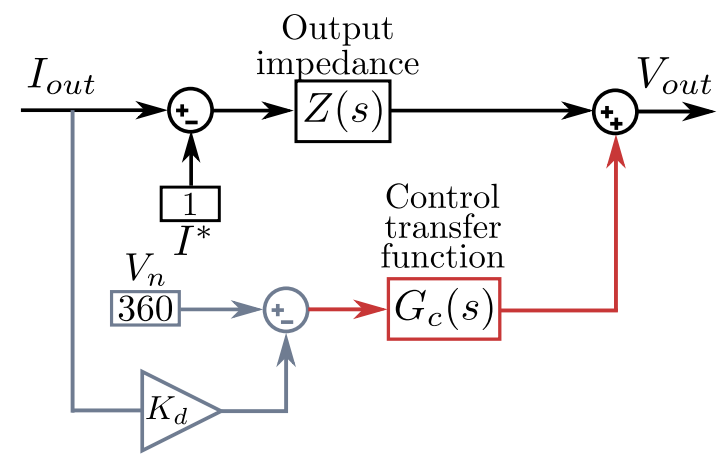

Figure 7.25: Sketch of the blackbox model of the dc-dc converter.

Table 7.1: Identified data of the blackbox models.

$$
\begin{array}{cc}
Z_{0.1 A}(s)= & Z_{1 A}(s)= \\
\frac{932.7 s+3.62 e-10}{s^{2}+10 s+1190} & \frac{802 s+6.64 e-10}{s^{2}+20.1 s+1129}
\end{array}
$$

\begin{tabular}{cc}
$G_{c 0.1 A}(s)=$ & $G_{c 1 A}(s)=$ \\
$\frac{1507 s+2.05 e 4}{s^{3}+29.15 s^{2}+139 s+2.05 e 4}$ & $\frac{2674 s+5.32 e 4}{s^{3}+61.7 s^{2}+212 s+5.32 e 4}$ \\
\hline$D W_{0.1 A}(s)=$ & $D W_{1 A}(s)=$ \\
$\frac{10}{s+10}$ & $\frac{5.14}{s+5.14}$ \\
\hline$Z(s)=$ & $G_{c}(s)=$ \\
$\frac{1402 s^{2}+3.03 e 5+2 e-3}{s^{3}+424 s^{2}+1.03 e 5 s+1.01 e 6}$ & $\frac{1.8 e 7}{s^{3}+619 s^{2}+1.6 e 5 s+1.8 e 7}$
\end{tabular}

conditions proposed. The integration of the series communication with the HiL program will be considered in future work. In this case, fixed values of the droop parameter were considered. The structure of the blackbox model identified is represented in Fig. 7.25 and the data is shown in Table 7.1.

In the next section the interconnection of the blackbox described above will be detailed and their performance will be analyzed in different scenarios.

\section{Case studies}

An interesting advantage of two-port models is that they can be combined in different ways to create more complex structures. In this section it will be shown how the interconnection of the individual blackbox models can be used to predict the interaction among the converters and their secondary controls, see Section 7.2. Finally, the simulation results will be validated by comparing them with the real equipment in the same situations. 


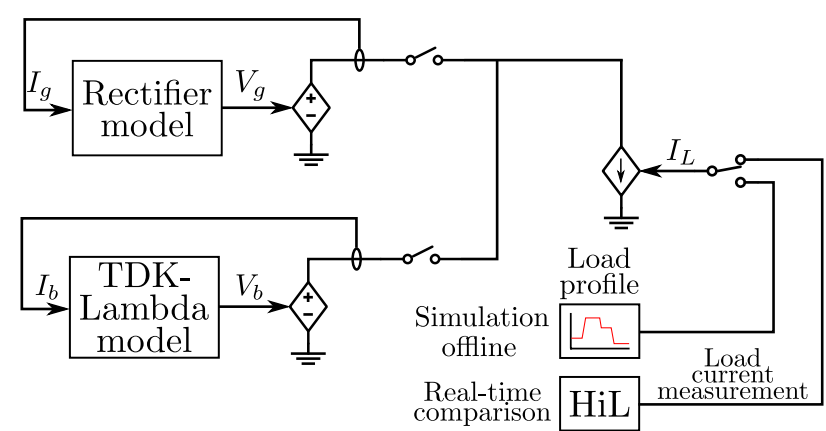

Figure 7.26: Interconnection of the blackbox models.

\section{Simulation Results}

The previous sections detailed how to obtain the blackbox models of the different converters used in this work. Now the goal is to use them to be able to simulate the behavior of the system shown in Fig. 7.22. The idea is to use the outputs of the models as references for controlled sources, which will be connected together to supply the loads. The input of the models, the output current in this case, will be fed back with a current measurement during the simulation. Thus, the only input of the model is the current demanded by the load. A sketch of the interconnection of the blackbox models identified is shown in Fig. 7.26. In this work the focus is on the dc bus, however it would be straightforward to extend this strategy to include the input port of the converters, using current sinks governed by the input current given by the G-parameters model, and measuring the input voltage to feed it back to the model. Those transfer functions have been excluded from this work for purpose of clearness. In addition, the series or parallel connection of further converters is also simple following the same idea.

\section{Interaction Among Converters}

The interconnected model detailed before will be used to study the behavior of the system under different circumstances. In Fig. 7.27, the dynamic of the signals is compared when only the grid interfacing converter supplies the load and when the battery interfacing converter is connected in parallel. This load profile will be used throughout all the experiments. It represents the different power requirements of a drilling vessel under various conditions as detailed in [124]. The power levels have been scaled to the power ratings of the converters available in the laboratory. The results show how the bus voltage experiences much higher oscillations when only the grid connected converter is supplying the load. At time $=8 \mathrm{~s}$, the second converter is connected and the bus voltage reduces considerably its fluctuation under load steps.

In this example the reference voltage of the battery converter was reduced to $345 \mathrm{~V}$, so it delivers current when the load is high and it stores energy when the load level is low. The droop parameters, $k_{d}$ were set to 5 and 9.3 for the rectifier and the TDK-Lambda, respectively. From this detailed information about the system dynamics, it would be simple to extract a estimation of the $\mathrm{SoC}$ (State of Charge) of the battery, as represented in the results, and the amount of energy that could be extracted during the transients of the system, which are commonly wasted in resistors banks. In this example, if we consider that $1 s$ corresponds to $1 h$, the battery shown would have a capacity of $2.4 \mathrm{kWh}$. The coulomb county method was used for the SoC estimation, which was considered to start at $50 \%$. The fluctuation of the $\mathrm{SoC}$ can be predicted based on the dynamic behavior of the system, which is a valuable information in order to calculate an optimal battery capacity. 

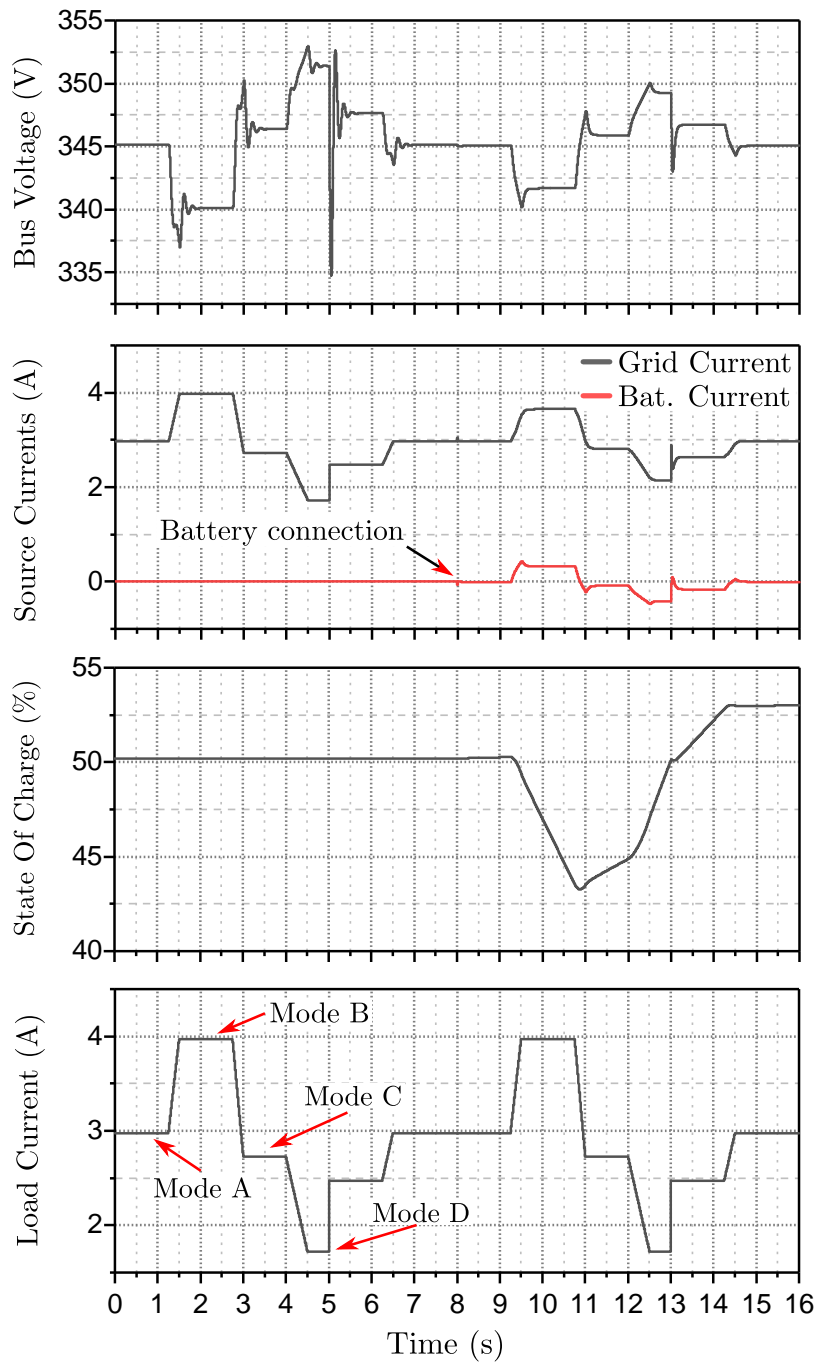

Figure 7.27: Simulation of how the microgrid dynamic behavior is affected by the parallel connection of the battery and grid interfacing converters.

\section{Effect of Secondary Control}

The next simulation shows how the behavior of the microgrid is affected when a secondary control is added. In this case, the secondary control shown in Fig. 7.24 is activated as a current sharing control. A PI controller is added to regulate the output current to be equal to the average of both source converters (see $(7.11)$ ). The proportional gain is set to $K_{p}=1$ and the integral gain $K_{i}$ is varied to three different values. In Fig. 7.28 it is shown that for $K_{i}=100$ the transitions are slow and without oscillations. For $K_{i}=1000$ the dynamic is faster and some oscillations start to appear and for $K_{i}=2000$ the oscillations become considerable. Notice that the model not only shows the expected higher oscillatory behavior when the gain is increased, but also a precise estimation of the actual behavior of the interconnected system, as it will be illustrated in the experimental validation. Therefore, the suitability of this model to design secondary controllers, as well as the possibility of checking the behavior of the converters arranged in different configurations and working in various conditions, is exposed. 

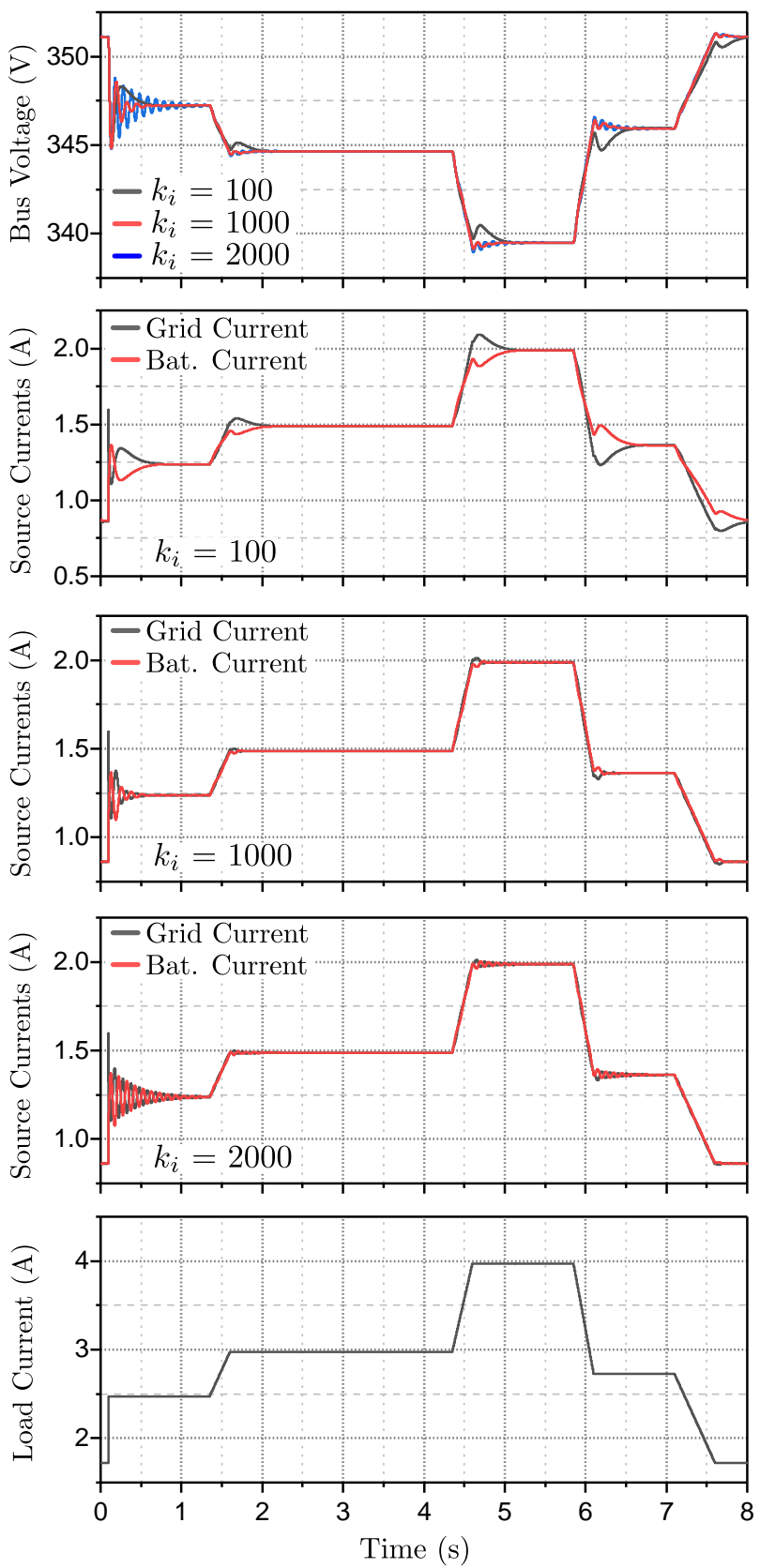

Figure 7.28: Simulation of how the microgrid dynamic behavior is affected by the design of the current sharing secondary control. 


\section{Experimental Validation}

The previous simulations results have been validated comparing the model behavior with the real hardware in similar conditions. In order to perform the comparison, the blackbox model was integrated in the HiL real-time simulator. The load profile was set into the electronic load and the only input of the blackbox model, $I_{L}$ from Fig. 7.26, was included as a measurement of the physical load current. Then the rest of the physical measurements are compared with the model results.

\section{Comparison between small-signal and large-signal models}

The first part of the validation compares the response of the small-signal models and the large-signal model with the setup response. These experiments show that the behavior of the system at low power differs from the behavior at medium and high power.

The first experiment depicts the response of the system when the current sharing control is activated at low power, see Fig. 7.29. It can be seen that the large-signal model follows the small-signal model identified at low power conditions and it is a good estimation of the setup behavior. Compared with the small-signal model identified at high power conditions there is a difference of $25 \%$ in the overshoot and twice the settling time of the bus voltage. Also the output currents of the battery and grid interface converters are compared with the small-signal models, but these comparisons are made separately for clarity.

The second experiment is performed in similar conditions but with a higher power demand from the load, see Fig. 7.30. Again the small-signal and large-signal models are compared with the measured signals from the setup when the current sharing control is activated. In this case, the large-signal model follows the small-signal model identified in high power conditions and it replicates the setup response with a good accuracy. Similarly, the setup behavior differs from the small-signal model obtained in low power conditions in around $25 \%$ of overshoot and half the settling time of the bus voltage. As in the previous case, the comparison of the output currents is made separately.

Having shown the suitability of the large-signal model to simulate the system, the next set of experiments will be focused on the capability of the model to include the effect of different system-level controllers and its comparison with the setup response.

\section{Droop Control}

The first comparison was made without a secondary control. As the dc source was unable to absorb energy, the voltage references of both converters were set to the same value $(360 \mathrm{~V})$. The droop parameters, $K_{d}$ were set to 5 and 9.3 for the grid and battery converters respectively. Fig 7.31 shows how the model is able to represent precisely the measurements.

\section{Current Sharing}

The next comparison was made adding a current sharing secondary control level (see (7.11)). The $k_{i}$ was set to 1000 , which was found as a good compromise between fast and low oscillations in the previous simulations. In Fig. 7.32 the comparison is shown, which includes the activation of the current sharing control loop. The results show how the activation of the current sharing provokes a drop in the bus voltage and it makes the system more oscillatory, as it was expected from the previous simulations. The agreement between model and measurements is very high.

\section{Voltage Restoration}

Finally, a voltage restoration secondary control was added instead of the current sharing control. A PI regulator has been used, comparing the bus voltage with the $360 \mathrm{~V}$ reference 

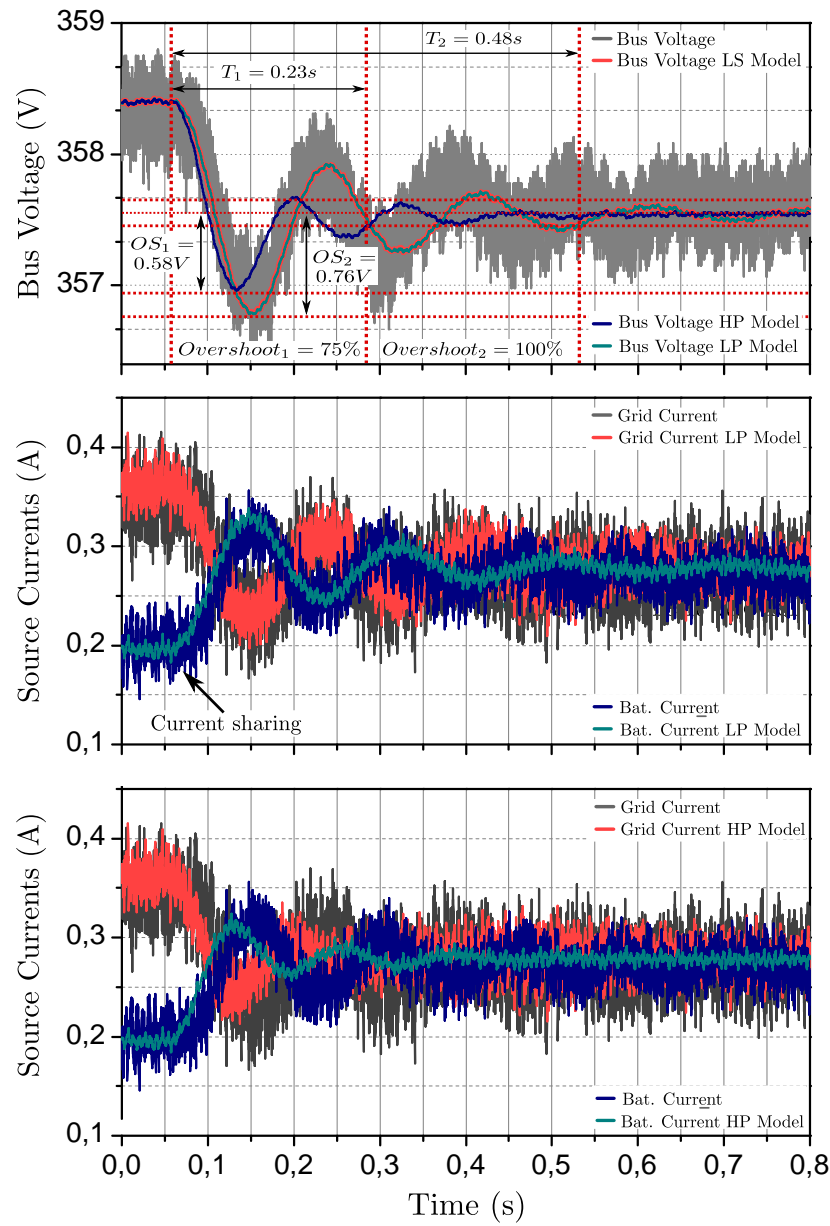

Figure 7.29: Comparison between the measured signals and the small-signal and the large-signal models response when the current sharing control is activated in low power conditions. LS: large-signal, HP: high power, LP: low power, OS: overshoot

(see (7.8)). The proportional gain was set to 1 and the integral gain was set to 10 . In Fig. 7.33 the activation of the voltage restoration is represented. As this control is only applied to the grid connected converter, most of the load current is supplied by this converter while using this strategy. Once more the model follows the measurements in all conditions. In the future work, the RS485 communication with the TDK converter will be integrated with the Dspace, so both converters can have the secondary control and share the load while performing voltage restoration.

\subsection{Conclusions}

In this chapter the blackbox modeling structures described in this thesis have been applied to converters which are integrated in a dc microgrid structure. First, the different models structures are classified according to their capability to account for different kind of nonlinearities in the dynamic behavior of the converter, which is a useful scheme for a system designer, who can select the simplest model able to follow accurately the dynamic behavior of the COTS converters. Second, the methodology to integrate individual blackbox models in any desired dc microgrid architecture is described. This approach uses controllable sources managed by the output variables of the models and measures the input variables resulting from the response of the rest of the system. This approach allows a straightfor- 

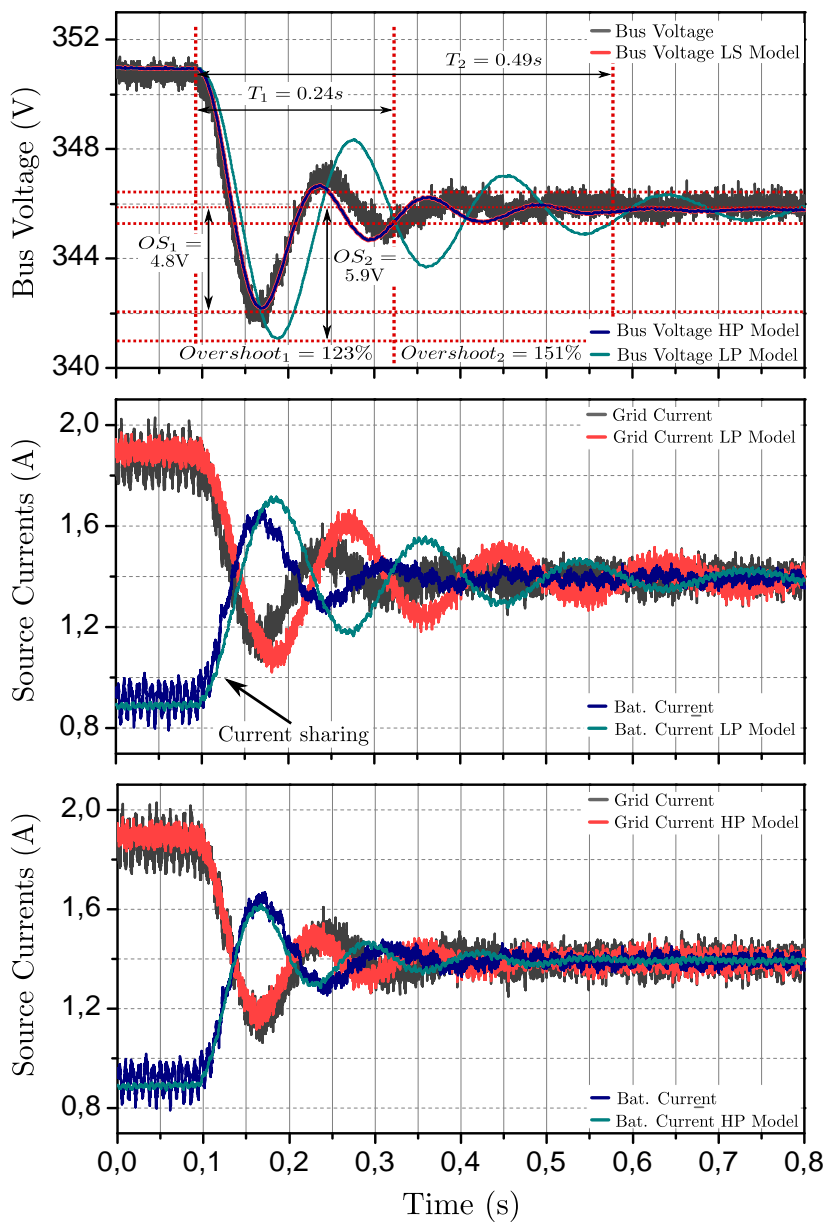

Figure 7.30: Comparison between the measured signals and the small-signal and the large-signal models response when the current sharing control is activated in medium power conditions. LS: large-signal, HP: high power, LP: low power, OS: overshoot.

ward inclusion of line impedances or external filters among the EPCs, so their effect can be analyzed or designed at simulation level. This strategy is considered interesting as it gives the designer an accurate estimation of the dynamic behavior of the system before it is physically implemented.

Afterwards, some examples of dc microgrids are presented. First the dynamic response of a dc microgrid that integrates sources converter regulating the bus voltage in parallel using droop controllers is shown. Additionally, the source converters include current limitations, which lead to strong nonlinearities in the dynamic behavior of the interconnected system. These sources supply POL converters connected to resistive loads. The approach to include droop controllers into the blackbox modeling structure is described. Simulation results are presented to validate the suitability of the proposed models to account for the phenomena detailed in a dc microgrid example.

Another dc microgrid example is included focusing on source converters with different control characteristics. Converters interfacing the grid, a renewable source, and a battery supplies three POL converters connected to variable resistive loads. The converter connected to the grid always works with droop control. The converter interfacing the renewable source has two modes of operation according to the power state of the microgrid, MPPT and droop controls. Finally, the converter connected to the battery works in cur- 

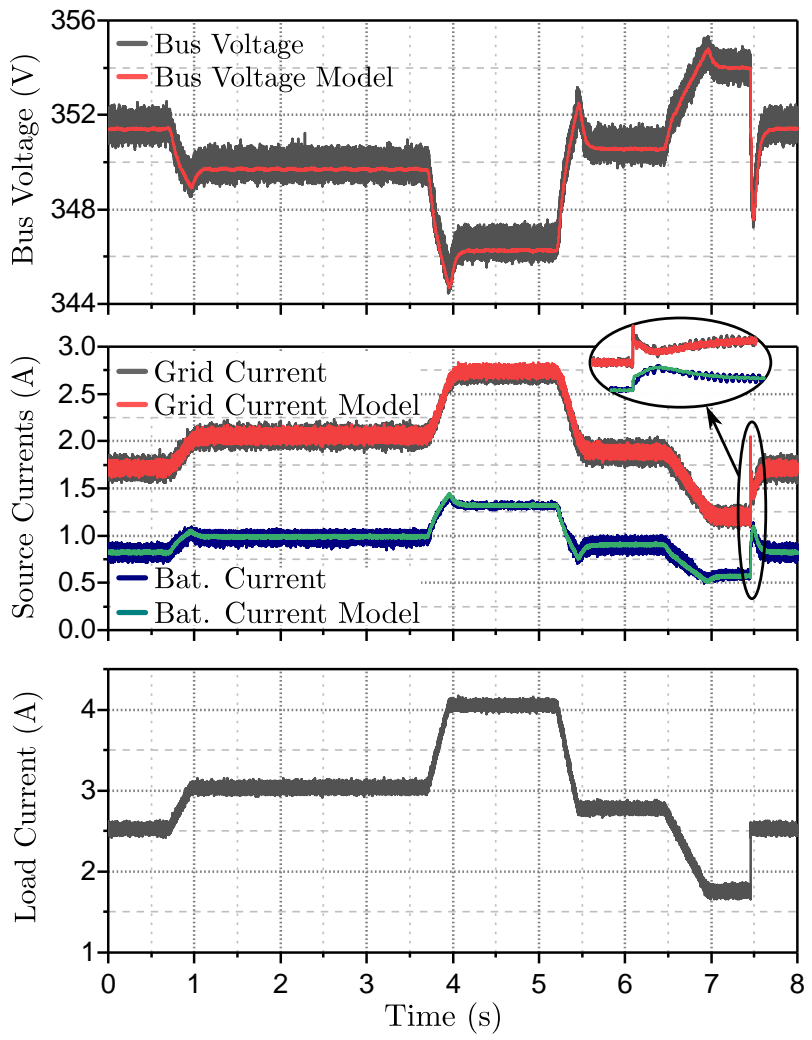

Figure 7.31: Experimental validation of the blackbox model of the microgrid using droop control.

rent mode. The blackbox models able to represent the dynamic behavior of the converter are first described individually and then interconnected in the dc microgrid. Furthermore, the inclusion of system-level controllers to control the current sharing and restore the bus voltage is also described and validated.

Finally, an experimental setup was implemented in order to validate the proposed blackbox modeling approach. The system integrates two parallel sources supplying an electronic load. First, the system is described and the identification of the models is detailed. Then, the interconnection of the blackbox models is described, as well as the strategy to perform simulations and to perform comparisons in real time with the real equipment. The inclusion of system-level controllers is also described and validated.

The results show the comparison between the real equipment and the blackbox model when the source converter works with droop control, when the current sharing control is activated, and when the voltage restoration control is applied. The dynamic behavior of the interconnected system when the load is changing in all modes is accurately estimated, as well as the dynamic behavior due to the activation of the system-level control strategies. It is worth noticing that the blackbox modeling approach is a very useful tool to design and tune system-level controllers. It has been shown that the parameters of the regulators are changed and the blackbox model estimation of its effect on the interconnected system is in very good agreement with the real system.

The proposed methodology to include the effect of system-level controllers in the large-signal blackbox models of the EPCs is an important contribution of this thesis. 

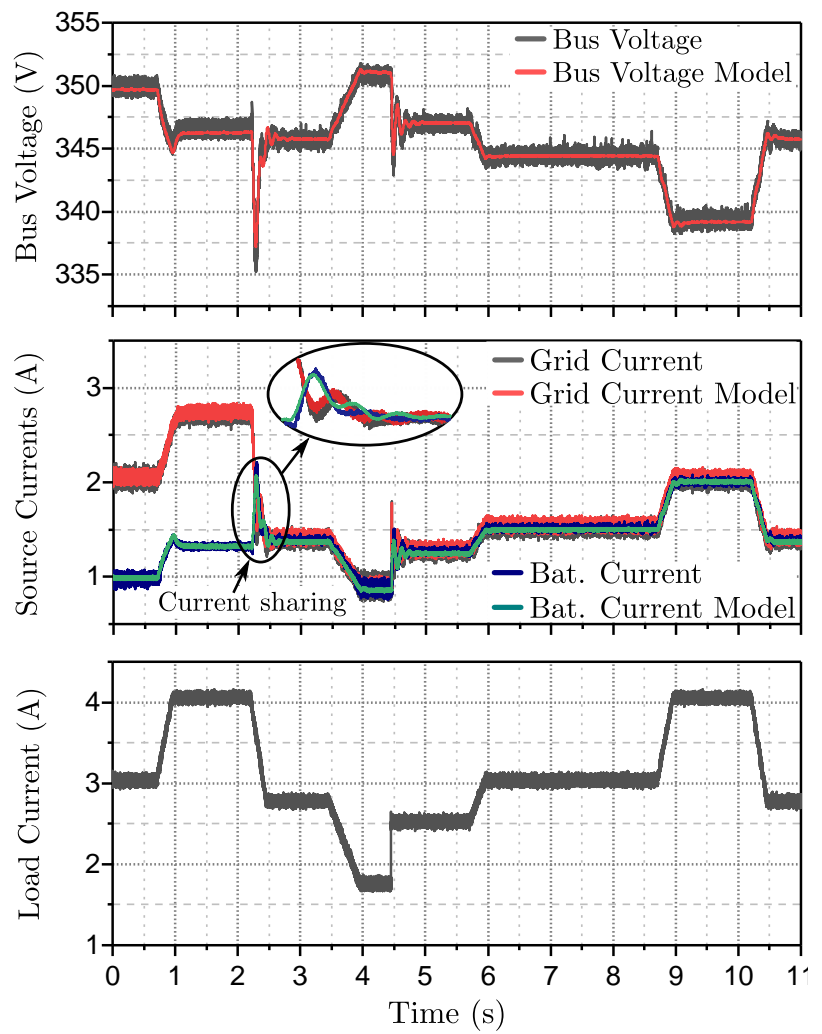

Figure 7.32: Experimental validation of the blackbox model of the microgrid in the activation of the current sharing. 

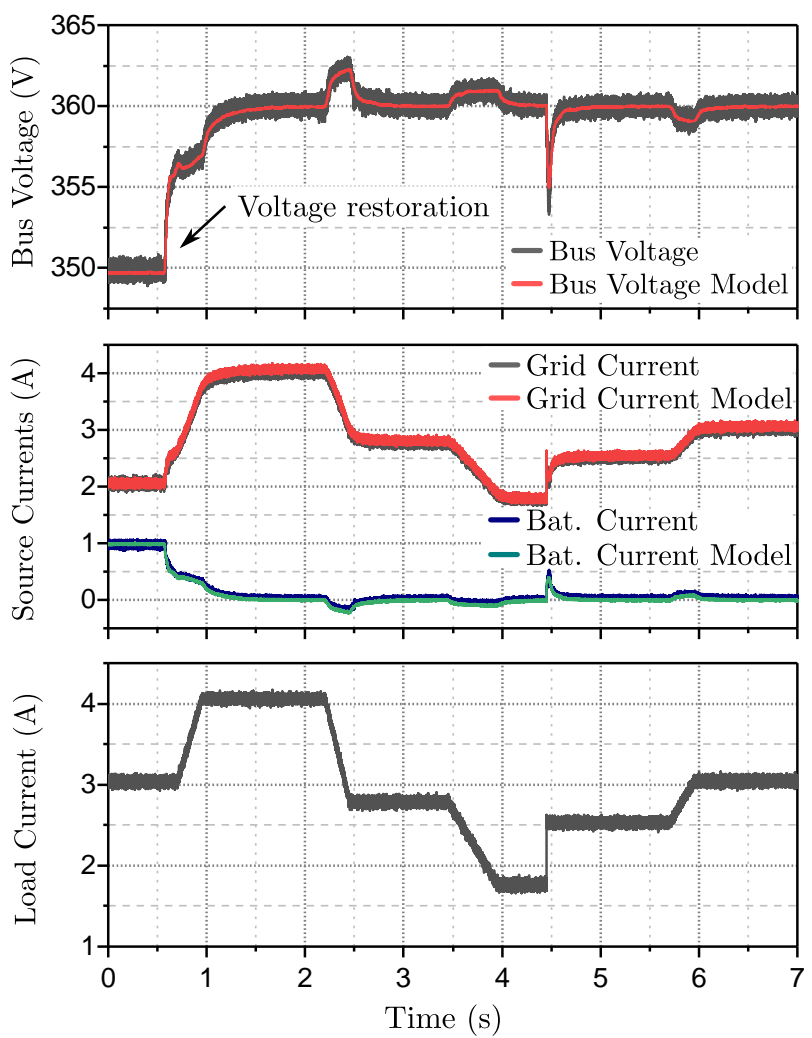

Figure 7.33: Experimental validation of the blackbox model of the microgrid in the activation of the voltage restoration. 



\section{Conclusions and Outlook}

$\mathrm{T}$

he main contribution of this thesis has been to provide a methodology to obtain blackbox models of electronic power converters with the capability of accounting for the particular phenomena characteristic of dc microgrids.

This thesis consists of two parts. The first part studies the background of the topic, including the introduction and the study of the state of the art. The second part assesses the large-signal blackbox modeling of electronic power converters in microgrids. First, the different blackbox modeling structures that can be found in the literature are compared. Second, a modified polytopic model is proposed in order to improve its accuracy. Third, a new blackbox modeling approach is proposed in order to account for the phenomena that the studied modeling approaches cannot reproduce. Fourth, a first approach towards a blackbox large signal stability analysis is proposed. Finally, the models analyzed are used to simulate dc microgrids in different scenarios and an experimental validation is presented.

In the introduction the motivation for the use of dc microgrids was detailed. Furthermore, the need for blackbox models able to reproduce the large-signal behavior of electronic power converters was justified. Also the reasons for the focus on a dc distribution were described.

The study of the state of the art included the architectures and control strategies proposed for dc microgrids. This analysis was used afterwards to identify the particular phenomena characteristic of dc microgrids that the models should be able to reproduce. Also the different modeling approaches for electronic power converters were reviewed, including both analytical and blackbox approaches. Despite the fact that the focus of this thesis is in blackbox approaches, it is considered that a review of analytical models is relevant because this kind of models have been extensively studied, so it is important to know them in order to have tools that could be implemented with a blackbox approach. Besides, some blackbox modeling techniques are based on their analytical counterparts, therefore knowing them can be also helpful to propose improvements to the blackbox structures. Finally, methodologies to assess the dynamic interactions among power converters and stability analyzes of this kind of systems were reviewed. The possibility of performing these analyzes using blackbox models has been studied in this work. This comprehensive review of the state of the art is considered the first contribution of this thesis.

In Chapter 3 the performance of the modeling approaches available in the literature are compared with different illustrative examples. This comparison includes the main char- 
acteristics of dc microgrids: wide range of operating points and controls with different operation modes. The second contribution of this thesis has been to identify the capabilities and limitations of the different modeling approaches. Furthermore, a modification of the Wiener-Hammerstein model has been proposed in order to account for converters with different operation modes. This analysis has been used to identify the cases that none of the models were able to reproduce, which are used as reference for the proposed models in the next chapters.

Chapter 4 proposes the use of dynamic weighting functions in the polytopic models. This approach improves the accuracy of the polytopic models with the classical weighting functions by taking into account the internal dynamic limitation of the converters to change their dynamic behavior. The proposed weighting functions use the poles of the system to limit the rate of change among small-signal models, which has been demonstrated to be a useful approach to model the converter when the input variables change with large and sharp steps. These steps are not unusual in dc microgrids due to the presence of resistive loads and sharp variation of the power delivered by renewable sources. However, it was also shown that in case the converters have strong nonlinearities this method can also result in a poor accuracy.

In order to account for the cases where the converters present strong nonlinearities and the input variables change sharply, a new modeling approach was proposed in Chapter 5. It consists in using parameter varying transfer functions, where instead of making a weighting combination of parallel small-signal models, it uses a single model with variable parameters. The variation of these parameters is also made with weighting functions depending on the operating points. The dynamic weighting functions are also useful in this modeling approach. This technique has the advantage that it takes into account the past values of the input and output variables. This approach was demonstrated to be able to account for the phenomena that none of the existing blackbox modeling approaches were able to reproduce with acceptable accuracy.

In Chapter 6 a first approach towards a blackbox large signal stability analysis was proposed. The use of small-signal stability analysis is very useful, but they are limited to linear systems or to perturbations around an operating point. As dc microgrids can work in a wide range of operating points and nonlinear behavior can be present, large signal stability analysis are relevant, for instance to estimate regions of attraction around equilibrium points. To the best of the author's knowledge, the blackbox large-signal stability analysis of dc microgrid-like system has not been studied in the literature. An important source of instability in microgrids is the constant power load behavior of tightly regulated point-ofload converters. This example has been taken into consideration and the proposed blackbox large signal stability method has been implemented and compared with the analytical result of a work found in the literature, with similar results. The method consists of using the local models to obtain a state-space representation of the system. A similarity transformation is used to refer the state variables with the output variables, which are the only information available about commercial converters. Then coincident models are derived, which share the same equilibrium point. Finally, the multi-model Lyapunov stability method is applied to the state matrices obtained to estimate the region of attraction around an equilibrium point.

Finally, in Chapter 7 the modeling of dc microgrids is studied. First, the methodology to interconnect individually identified blackbox models into a dc microgrid structure is detailed. Then methods to include droop and system-level controllers into the blackbox modeling structures are proposed. With these tools and the modeling structures studied in previous chapters, different dc microgrids are considered. The first example considers source converters regulating in parallel the bus voltage by means of droop controllers and the effect of the current limitation implemented in the control of the source converters. 
The second example focuses on the control characteristics of the converters interfacing different elements, as renewable sources, the grid, and batteries. These converters work with droop controllers, maximum-power-point-tracking control, and current controls. Besides, the inclusion of system-level controllers is studied. The methodology to account for these phenomena is detailed and validated.

Afterwards, an experimental validation is presented. The system consists of two sources regulating the bus voltage in parallel with droop controllers and a electronic load with a variable load profile. The system has been described and the identified models detailed. Furthermore, the inclusion of system-level controllers is studied. The system-level control is designed using the blackbox models and then implemented in the real system. The blackbox model is compared with the real system in real time, using a hardware-in-theloop equipment, and the performance of the model to reproduce the dynamic behavior of the interconnected model in all cases is highly accurate.

In summary, this thesis has identified the characteristics of dc microgrids and the different modeling approaches have been compared and their capability to account for these particular phenomena has been evaluated. From this analysis the limitations of the existing modeling structures have been identified and new blackbox modeling approaches have been proposed, which are able to reproduce these cases. Besides, a first approach towards a large signal stability analysis has been proposed. Finally, methods for the interconnection of blackbox models and the inclusion of system-level controllers have been proposed and validated with dc microgrid examples both at simulation and experimental levels.

As it has been justified along the thesis, this methodology provides the system designer of dc microgrids the possibility to perform simulations of the dynamic response of dc power distribution architectures consisting of commercial-off-the-shelf converters. These models are able to account for the characteristic phenomena of the dc microgrids. Hence, they can be used to check the compliance of the system with the standards, to design external filters if necessary, to check the effect of line impedances, to design protections and system-level controllers, among other possibilities. This possibility is important to reduce the risk of unexpected failures in the physical installation and to reduce the time of the experimental tests. Consequently, the commissioning time and cost is reduced.

\subsection{Summary of contributions}

The contributions of this thesis are:

- A thorough review about the topics related to this thesis, such as: architectures and control techniques for dc microgrids; analytical and blackbox modeling techniques for dc EPCs as well as for complex systems such as dc microgrids; and dynamic interaction and stability analysis of dc EPCs and dc distribution systems.

- A comparison among the existing blackbox modeling techniques for dc EPCs through various illustrative examples, including the three basic topologies of non-isolated dc EPCs. Their advantages and disadvantages and their capabilities and limitations have been discussed in detail.

- The concept of dynamic weighting functions has been proposed in order to improve the accuracy of the polytopic models. This technique takes into account the dynamic of the transition among local models, which has been related with the poles of the system.

- A new modeling approach has been proposed in order to obtain very accurate blackbox models of EPCs with strong nonlinearities. This approach is based on the polytopic 
model, but instead of using a collection of local models, parameter varying transfer functions are implemented. The main advantage of this model is that it takes into account the previous values of the input and output signals, avoiding the problem of initial conditions of the polytopic models.

- A first approach towards a methodology to perform blackbox nonlinear stability analyzes of dc microgrids. A system with a constant power load has been considered and the region of asymptotic stability around an equilibrium point was obtained using the Lyapunov method for multimodel systems. The result was equivalent to the one obtained analytically in a reference paper.

- Different alternatives to include the effect of droop controllers in the dynamic response of the EPCs: the inclusion of its static effect and the inclusion of its static and dynamic effect.

- A methodology to account for the dynamic behavior of converters with different control modes using blackbox models. Converters with current limitations, which change from voltage to current mode; and converters which changes from MPPT control to voltage mode according to their output voltage (DBS control technique) have been considered.

- A methodology to include the effect of system-level controllers in the dynamic behavior of dc microgrids. Secondary control strategies, such as current sharing or voltage restoration, have been implemented in the source converters of dc microgrids both at simulation level and experimentally, resulting in very accurate estimations of the response of the system.

\subsection{Future Work}

The line of research started with this thesis will be continued focusing in different aspects. First, other kinds of nonlinearities could be considered as converters with nonlinear regulators, nonlinear loads, or converters with other topologies. Besides, a more extensive experimental validation, considering more complex systems or converters with higher power levels could be performed. Also, the effect of the start-up strategies or more detailed protection systems can be further studied. Furthermore, the use of the blackbox models to monitor the dynamic behavior of the system during operation in order to anticipate failures could be also considered. Another possible line of research is to study the feasibility of performing an on-line identification of the system, i.e. obtaining a model of the system which is under operation. The application of the proposed blackbox modeling techniques to ac microgrids is also a possible research line.

Another interesting line of research is the automation of the identification process. The idea is to create a system able to perform the tests to the converters around different operating points, according to the information provided by the user. From the response of the converter to these perturbations identify the transfer functions of the model. Afterwards, integrate these transfer functions in the large-signal modeling structures studied in this thesis. Finally, the system should compare the performance of the model with the response of the converter to different steps. In case the model is not accurate enough, the system should modify it according to a predefined strategy. In this thesis the methods have been proposed with a deterministic approach, so they are very suitable to be implemented in a automated process. Indeed, as a result of the work performed in this thesis, a new project was proposed and accepted to research the possibility of creating this automated system able to generate blackbox models of commercial-off-the-shelf converters.

Finally, the methodology to perform large-signal stability analysis using identified models will be tested in more complex scenarios. The analysis of a similar system with more 
than one constant power load could be the first approach, whereas the addition of more than one source and different operation modes could be a next step. Also the study of other types of Lyapunov functions aiming to reduce the conservativeness of this approach can be a future research line.

\subsection{Dissemination}

\subsubsection{Direct Contributions}

\section{Articles}

- A. Frances, R. Asensi, O. Garcia, R. Prieto and J. Uceda, "Modeling Electronic Power Converters in Smart DC Microgrids - An Overview," in IEEE Transactions on Smart Grid.

- A. Frances, A. Anvari-Moghaddam, E. Rodriguez-Diaz, J. C. Vasquez, J. M. Guerrero and J. Uceda, "Dynamic Assessment of COTS Converters-based DC Integrated Power Systems in Electric Ships," in IEEE Transactions on Industrial Informatics.

\section{Conference papers}

- A. Frances, R. Asensi, O. Garcia, R. Prieto and J. Uceda, "A black-box modeling approach for DC nanogrids," 2016 IEEE Applied Power Electronics Conference and Exposition (APEC), Long Beach, CA, 2016, pp. 1624-1631.

- A. Frances, R. Asensi, O. Garcia and J. Uceda, "A blackbox large signal Lyapunovbased stability analysis method for power converter-based systems," 2016 IEEE 17th Workshop on Control and Modeling for Power Electronics (COMPEL), Trondheim, 2016, pp. 1-6.

- A. Frances, R. Asensi, O. Garcia, R. Prieto and J. Uceda, "The performance of polytopic models in smart DC microgrids," 2016 IEEE Energy Conversion Congress and Exposition (ECCE), Milwaukee, WI, 2016, pp. 1-8.

- A. Frances, R. Asensi, O. Garcia, R. Prieto and J. Uceda, "How to model a DC microgrid: Towards an automated solution," 2017 IEEE Second International Conference on DC Microgrids (ICDCM), Nuremburg, 2017, pp. 609-616.

\subsubsection{Other Works and Collaborations}

\section{Conference papers}

- G. Guarderas, A. Frances, R. Asensi and J. Uceda, "Large-signal black-box behavioral modeling of grid-supporting power converters in AC microgrids," 2017 IEEE 6th International Conference on Renewable Energy Research and Applications (ICRERA), San Diego, CA, 2017, pp. 153-158.

- K. Martin, M. R. Rogina, A. Vazquez, D. G. Lamar, A. Frances, R. Asensi, J. Sebastian, J. Uceda, "Distribucion con Nano-Redes de Corriente Continua en el Hogar", 2016 XXIII Seminario Anual de Automática, Electrónica Industrial e Instrumentación (SAAEI'16), Elche (España). 


\section{Projects}

- Arquitecturas de nano-redes inteligentes de energía eléctrica en edificios (ANRI). Supported by the Spanish Ministry of Economy and Competitiveness, under the research grant no: DPI2013-47176-C2-1-12.

- Sistema de identificación automática de modelos no lineales en caja negra de convertidores electrónicos de potencia en micro-redes inteligentes de corriente continua (IDENMRED). Supported by the Spanish Ministry of Economy and Competitiveness, under the research grant no: DPI2016-78644-P. 
Part III

Appendices 



\section{Series connection of electronic power converters}

The two-port models of the individual EPCs (Fig. A.1) are defined as:

$$
\begin{gathered}
v_{o}^{1}=G_{1} v_{i n}^{1}-Z_{1} i_{o}^{1} \\
i_{i n}^{1}=Y_{1} v_{i n}^{1}+H_{1} i_{o}^{1} \\
v_{o}^{2}=G_{2} v_{i n}^{2}-Z_{2} i_{o}^{2} \\
i_{i n}^{2}=Y_{2} v_{i n}^{2}+H_{2} i_{o}^{2}
\end{gathered}
$$

In order to obtain the equations of the series-connected system the following relationships are considered:

$$
\begin{aligned}
v_{o}^{1} & =v_{i n}^{2} \\
i_{o}^{1} & =i_{i n}^{2}
\end{aligned}
$$

The resulting equation for the voltage at the interconnection interface is:

$$
v_{o}^{1}=G_{1} v_{i n}^{1}-Z_{1}\left(Y_{2} v_{o}^{1}+H_{2} i_{o}^{2}\right)
$$

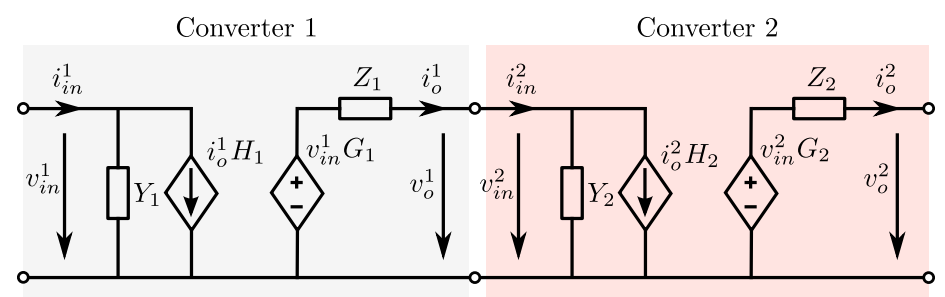

Figure A.1: Individual G-parameters models of two converters connected in series. 
Rearranging terms the following expression is obtained:

$$
v_{o}^{1}=\frac{G_{1}}{1+Z_{1} Y_{2}} v_{i n}^{1}-\frac{Z_{1} H_{2}}{1+Z_{1} Y_{2}} i_{o}^{2}
$$

Now the expression of the output voltage of the load converter as a function of the input voltage of the sources converter and the output current of the load converter would be as follows:

$$
v_{o}^{2}=G_{2} v_{o}^{1}-Z_{2} i_{o}^{2}
$$

which, using A.5 and rearranging terms, results in:

$$
v_{o}^{2}=\frac{G_{1} G_{2}}{1+Z_{1} Y_{2}} v_{i n}^{1}-\frac{Z_{2}+Z_{1}\left(Y_{2} Z_{2}+H_{2} G_{2}\right)}{1+Z_{1} Y_{2}} i_{o}^{2}
$$

Similarly, the expression of the input current of the source converter as a function of the input voltage of the source converter and the current of the load converter would be:

$$
i_{i n}^{1}=Y_{1} v_{i n}^{1}+H_{1} i_{i n}^{2}
$$

and using A.2 and A.3 the following equation is obtained:

$$
i_{i n}^{1}=Y_{1} v_{i n}^{1}+H_{1}\left(Y_{2} v_{o}^{1}+H_{2} i_{o}^{2}\right)
$$

Now using A.5 and rearranging terms the following expression can be derived:

$$
i_{i n}^{1}=\frac{Y_{1}+Y_{2}\left(Y_{1} Z_{1}+H_{1} G_{1}\right)}{1+Z_{1} Y_{2}} v_{i n}^{1}+\frac{H_{1} H_{2}}{1+Z_{1} Y_{2}} i_{o}^{2}
$$

Therefore the two-port model of the series-interconnected system is:

$$
\left(\begin{array}{c}
v_{o}^{2} \\
i_{\text {in }}^{1}
\end{array}\right)=\left(\begin{array}{cc}
\frac{G_{1} G_{2}}{1+Z_{1} Y_{2}} & -\frac{Z_{2}+Z_{1}\left(Y_{2} Z_{2}+H_{2} G_{2}\right)}{1+Z_{1} Y_{2}} \\
\frac{Y_{1}+Y_{2}\left(Y_{1} Z_{1}+H_{1} G_{1}\right)}{1+Z_{1} Y_{2}} & \frac{H_{1} H_{2}}{1+Z_{1} Y_{2}}
\end{array}\right)\left(\begin{array}{c}
v_{i n}^{1} \\
\\
i_{o}^{2}
\end{array}\right)
$$




\section{Analytical G-parameters model}

The G-parameters model is an input-output representation of a two-port system where the input variables are the input voltage and the output current, and the output variables are the output voltage and the input current. It consists of four transfer functions that can be derived from the state-space representation of the system:

$$
\begin{aligned}
& \dot{x}(t)=A x(t)+B u(t) \\
& y(t)=C x(t)+D u(t)
\end{aligned}
$$

where $x$ is the state vector, $A$ is the state matrix, $B$ is the input matrix, $u$ is the input vector, $y$ is the output vector, $C$ is the output matrix, and $D$ is the feed-through matrix. In order to obtain the input-output representation, the Laplace transform can be applied to isolate $x$ as a function of the input vector, and substitute it in the second equation:

$$
\begin{aligned}
s x(s) & =A x(s)+B u(s) \\
(s I-A) x(s) & =B u(s) \\
x(s) & =(s I-A)^{-1} B u(s)
\end{aligned}
$$

where $I$ is the identity matrix. Substituting $x(s)$ in the second equations of (B.1) the input-output representation is:

$$
\begin{aligned}
& y(s)=C(s I-A)^{-1} B u(s)+D u(s) \\
& y(s)=\left(C(s I-A)^{-1} B+D\right) u(s)
\end{aligned}
$$

therefore, the relationship between the state space matrices and the G-parameters transfer functions is:

$$
\left(\begin{array}{cc}
G(s) & -Z(s) \\
Y(s) & H(s)
\end{array}\right)=C(s I-A)^{-1} B+D
$$

or equivalently, using the relationship of the inverse of a matrix with its adjugate: 


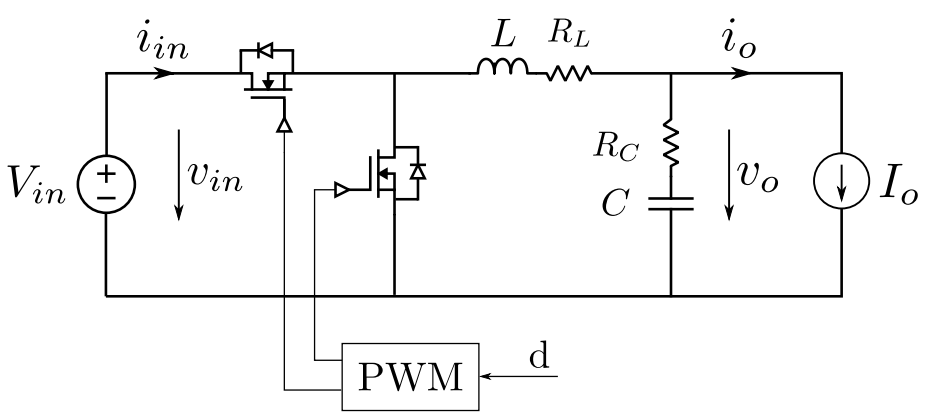

Figure B.1: Synchronous buck converter circuit in open loop connected to a constant current load.

$$
\left(\begin{array}{cc}
G(s) & -Z(s) \\
Y(s) & H(s)
\end{array}\right)=\frac{1}{\operatorname{det}(s I-A)} \operatorname{Cadj}(s I-A) B+D
$$

where $\operatorname{adj}(\cdot)$ represents the adjugate of a matrix and $\operatorname{det}(\cdot)$ represents the determinant of a matrix.

\section{B.1 Synchronous buck converter}

In this section the G-parameters model of the buck converter shown in Fig. B.1 as a function of the state-space matrices is represented. The state space representation of this converter in open loop is:

$$
\begin{aligned}
& \left(\begin{array}{c}
\frac{d v_{c}}{d t} \\
\frac{d i_{L}}{d t}
\end{array}\right)=\left(\begin{array}{cc}
0 & \frac{1}{C} \\
\frac{-1}{L} & \frac{-\left(R_{L}+R_{c}\right)}{L}
\end{array}\right)\left(\begin{array}{c}
v_{c} \\
i_{L}
\end{array}\right)+\left(\begin{array}{cc}
0 & \frac{-1}{C} \\
\frac{D}{L} & \frac{R_{c}}{L}
\end{array}\right)\left(\begin{array}{c}
v_{i n} \\
i_{o}
\end{array}\right) \\
& \left(\begin{array}{c}
v_{o} \\
i_{i n}
\end{array}\right)=\left(\begin{array}{cc}
1 & R_{c} \\
0 & D
\end{array}\right)\left(\begin{array}{c}
v_{c} \\
i_{L}
\end{array}\right)+\left(\begin{array}{cc}
0 & -R_{c} \\
0 & 0
\end{array}\right)\left(\begin{array}{c}
v_{i n} \\
i_{o}
\end{array}\right)
\end{aligned}
$$

The characteristic polynomial given by $\operatorname{det}(s I-A)$ will define the poles of the transfer functions of the four G-parameters:

$$
\operatorname{det}(s I-A)=s^{2}+\frac{R_{L}+R_{c}}{L} s+\frac{1}{L C}
$$

The zeros of the G-parameters will be given by:

$$
\operatorname{Cadj}(s I-A) B+\operatorname{Ddet}(s I-A)
$$

where

$$
\operatorname{adj}(s I-A)=\left(\begin{array}{cc}
s+\frac{R_{L}+R_{c}}{L} & \frac{1}{C} \\
\frac{-1}{L} & s
\end{array}\right)
$$


Substituting (B.9) and (B.7) in (B.8), the zeros of the G-parameters can be expressed as:

$$
\left(\begin{array}{cc}
1 & R_{c} \\
0 & d
\end{array}\right)\left(\begin{array}{cc}
s+\frac{R_{L}+R_{c}}{L} & \frac{1}{C} \\
\frac{-1}{L} & s
\end{array}\right)\left(\begin{array}{cc}
0 & \frac{-1}{C} \\
\frac{d}{L} & \frac{R_{c}}{L}
\end{array}\right)+\left(\begin{array}{cc}
0 & -R_{c} \\
0 & 0
\end{array}\right)\left(s^{2}+\frac{R_{L}+R_{c}}{L} s+\frac{1}{L C}\right)
$$

Finally, evaluating (B.10) and (B.7) in (B.5) the transfer functions of the G-parameters can be expressed as:

$$
\begin{aligned}
& G(s)=\frac{D}{L C} \frac{R_{c} C s+1}{s^{2}+\frac{R_{L}+R_{c}}{L} s+\frac{1}{L C}} \\
& Z(s)=\frac{R_{c} s^{2}+\left(\frac{L+R_{c} L C\left(R_{L}+R_{c}\right)-R_{c}^{2}}{L C}\right) s+\frac{R_{L}}{L C}}{s^{2}+\frac{R_{L}+R_{c}}{L} s+\frac{1}{L C}} \\
& Y(s)=\frac{D^{2}}{L} \frac{s}{s^{2}+\frac{R_{L}+R_{c}}{L} s+\frac{1}{L C}} \\
& H(s)=\frac{D}{L C} \frac{R_{c} C s+1}{s^{2}+\frac{R_{L}+R_{c}}{L} s+\frac{1}{L C}}
\end{aligned}
$$

The natural frequency, $\omega_{n}$, and the damping coefficient, $\xi$, can be expressed as:

$$
\begin{aligned}
\omega_{n} & =\frac{1}{\sqrt{L C}} \\
\xi & =\frac{R_{L}+R_{c}}{2} \sqrt{\frac{C}{L}}
\end{aligned}
$$

In Fig. B.2 the frequency response of the G-parameters for different values of the duty cycle is presented. The parameters evaluated are the ones specified in Fig. 3.1.

\section{B.2 Synchronous boost converter}

In this section the G-parameters model of the boost converter shown in Fig. B.3 as a function of the state-space matrices is represented. The state space representation is of this converter in open loop is: 

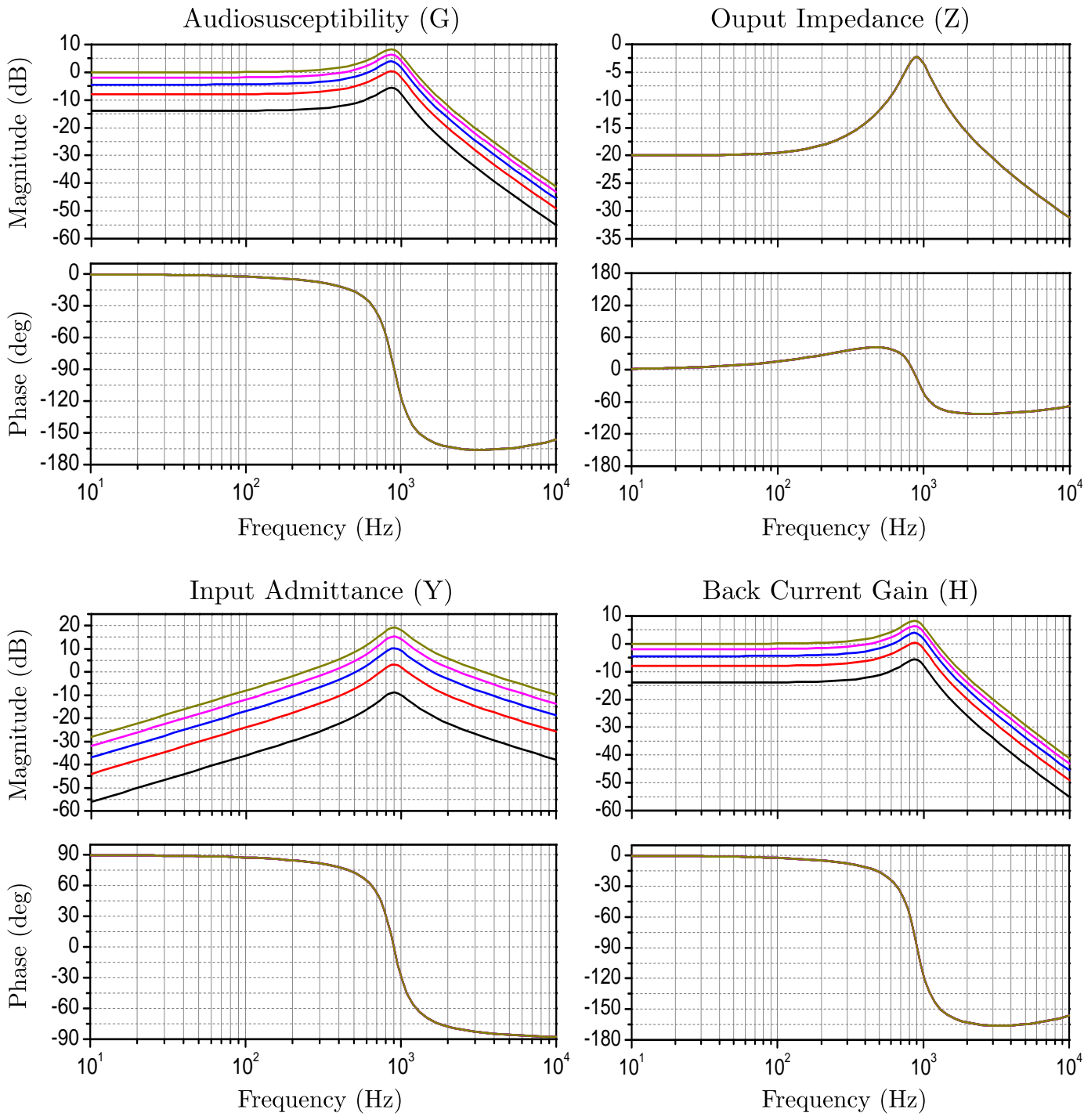

$$
-\mathrm{d}=0.2
$$

$-\mathrm{d}=0.4$

$-\mathrm{d}=0.6$

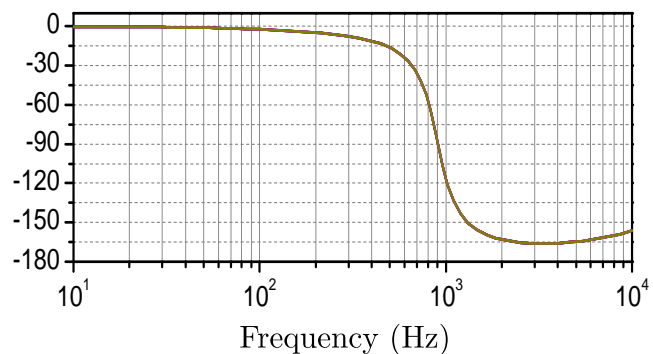

Figure B.2: Variation of the frequency response of the G-parameters of the synchronous buck converter for different values of the duty cycle.

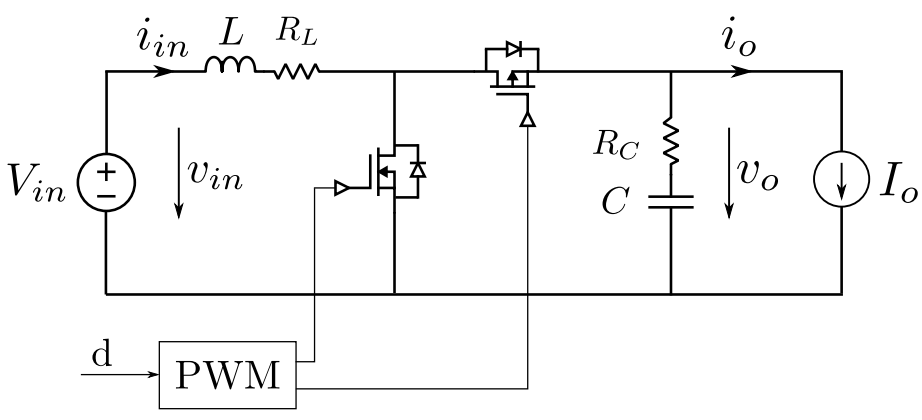

Figure B.3: Synchronous boost converter circuit. 


$$
\begin{aligned}
\left(\begin{array}{c}
\frac{d v_{c}}{d t} \\
\frac{d i_{L}}{d t}
\end{array}\right)=\left(\begin{array}{cc}
0 & \frac{1-D}{C} \\
\frac{-(1-D)}{L} & \frac{-\left(R_{L}+(1-D) R_{c}\right)}{L}
\end{array}\right)\left(\begin{array}{c}
v_{c} \\
i_{L}
\end{array}\right)+\left(\begin{array}{cc}
0 & \frac{-1}{C} \\
\frac{1}{L} & \frac{(1-D) R_{c}}{L}
\end{array}\right)\left(\begin{array}{c}
v_{\text {in }} \\
i_{o}
\end{array}\right) \\
\left(\begin{array}{c}
v_{o} \\
i_{\text {in }}
\end{array}\right)=\left(\begin{array}{cc}
1 & (1-D) R_{c} \\
0 & 1
\end{array}\right)\left(\begin{array}{c}
v_{c} \\
i_{L}
\end{array}\right)+\left(\begin{array}{cc}
0 & -R_{c} \\
0 & 0
\end{array}\right)\left(\begin{array}{c}
v_{i n} \\
i_{o}
\end{array}\right)
\end{aligned}
$$

The characteristic polynomial given by $\operatorname{det}(s I-A)$ will define the poles of the transfer functions of the four G-parameters:

$$
\operatorname{det}(s I-A)=s^{2}+\frac{R_{L}+(1-D) R_{c}}{L} s+\frac{(1-D)^{2}}{L C}
$$

The zeros of the G-parameters are given by (B.8), where:

$$
\operatorname{adj}(s I-A)=\left(\begin{array}{cc}
s+\frac{R_{L}+(1-D) R_{c}}{L} & \frac{1-D}{C} \\
\frac{-(1-D)}{L} & s
\end{array}\right)
$$

Substituting (B.15) and (B.14) in (B.8), the zeros of the G-parameters can be expressed as:

$$
\begin{aligned}
& \left(\begin{array}{cc}
1 & (1-D) R_{c} \\
0 & 1
\end{array}\right)\left(\begin{array}{cc}
s+\frac{R_{L}+(1-D) R_{c}}{L} & \frac{1-D}{C} \\
\frac{-(1-D)}{L} & s
\end{array}\right)\left(\begin{array}{cc}
0 & \frac{-1}{C} \\
\frac{1}{L} & \frac{(1-D) R_{c}}{L}
\end{array}\right)+ \\
& +\left(\begin{array}{cc}
0 & -R_{c} \\
0 & 0
\end{array}\right)\left(s^{2}+\frac{R_{L}+(1-D) R_{c}}{L} s+\frac{(1-D)^{2}}{L C}\right)
\end{aligned}
$$

Finally, evaluating (B.16) and (B.14) in (B.5) the transfer functions of the G-parameters can be expressed as: 


$$
\begin{aligned}
& G(s)=\frac{1-D}{L C} \frac{R_{c} C s+1}{s^{2}+\frac{R_{L}+(1-D) R_{c}}{L} s+\frac{(1-D)^{2}}{L C}} \\
& Z(s)=\frac{R_{c} s^{2}+\frac{L+R_{c} C\left(R_{L}+R_{c}(1-D) D\right)}{L C} s+\frac{R_{L}+R_{c}(1-D) D}{L C}}{s^{2}+\frac{R_{L}+(1-D) R_{c}}{L} s+\frac{(1-D)^{2}}{L C}} \\
& Y(s)=\frac{1}{L} \frac{R^{2}}{s^{2}+\frac{R_{L}+(1-D) R_{c}}{L} s+\frac{(1-D)^{2}}{L C}} \\
& H(s)=\frac{1-D}{L C} \frac{R_{c} C s+1}{L}
\end{aligned}
$$

The natural frequency, $\omega_{n}$, and the damping coefficient, $\xi$, can be expressed as:

$$
\begin{aligned}
\omega_{n} & =\frac{1-D}{\sqrt{L C}} \\
\xi & =\frac{1}{2}\left(\frac{R_{L}}{1-D}+R_{c}\right) \sqrt{\frac{C}{L}}
\end{aligned}
$$

In Fig. B.4 the frequency response of the G-parameters for different values of the duty cycle is presented. The parameters evaluated are the ones specified in Fig. 3.11.

\section{B.3 Synchronous buck-boost converter}

In this section the G-parameters model of the buck-boost converter shown in Fig. B.5 as a function of the state-space matrices is represented. The state space representation of this converter in open loop is:

$$
\begin{aligned}
& \left(\begin{array}{c}
\frac{d v_{c}}{d t} \\
\frac{d i_{L}}{d t}
\end{array}\right)=\left(\begin{array}{cc}
0 & \frac{-(1-D)}{C} \\
\frac{1-D}{L} & \frac{-\left(R_{L}+(1-D) R_{c}\right)}{L}
\end{array}\right)\left(\begin{array}{c}
v_{c} \\
i_{L}
\end{array}\right)+\left(\begin{array}{cc}
0 & \frac{-1}{C} \\
\frac{D}{L} & \frac{-(1-D) R_{c}}{L}
\end{array}\right)\left(\begin{array}{c}
v_{i n} \\
i_{o}
\end{array}\right) \\
& \left(\begin{array}{c}
v_{o} \\
i_{\text {in }}
\end{array}\right)=\left(\begin{array}{cc}
1 & -(1-D) R_{c} \\
0 & D
\end{array}\right)\left(\begin{array}{c}
v_{c} \\
i_{L}
\end{array}\right)+\left(\begin{array}{cc}
0 & -R_{c} \\
0 & 0
\end{array}\right)\left(\begin{array}{c}
v_{i n} \\
i_{o}
\end{array}\right)
\end{aligned}
$$

The characteristic polynomial given by $\operatorname{det}(s I-A)$ will define the poles of the transfer functions of the four G-parameters: 

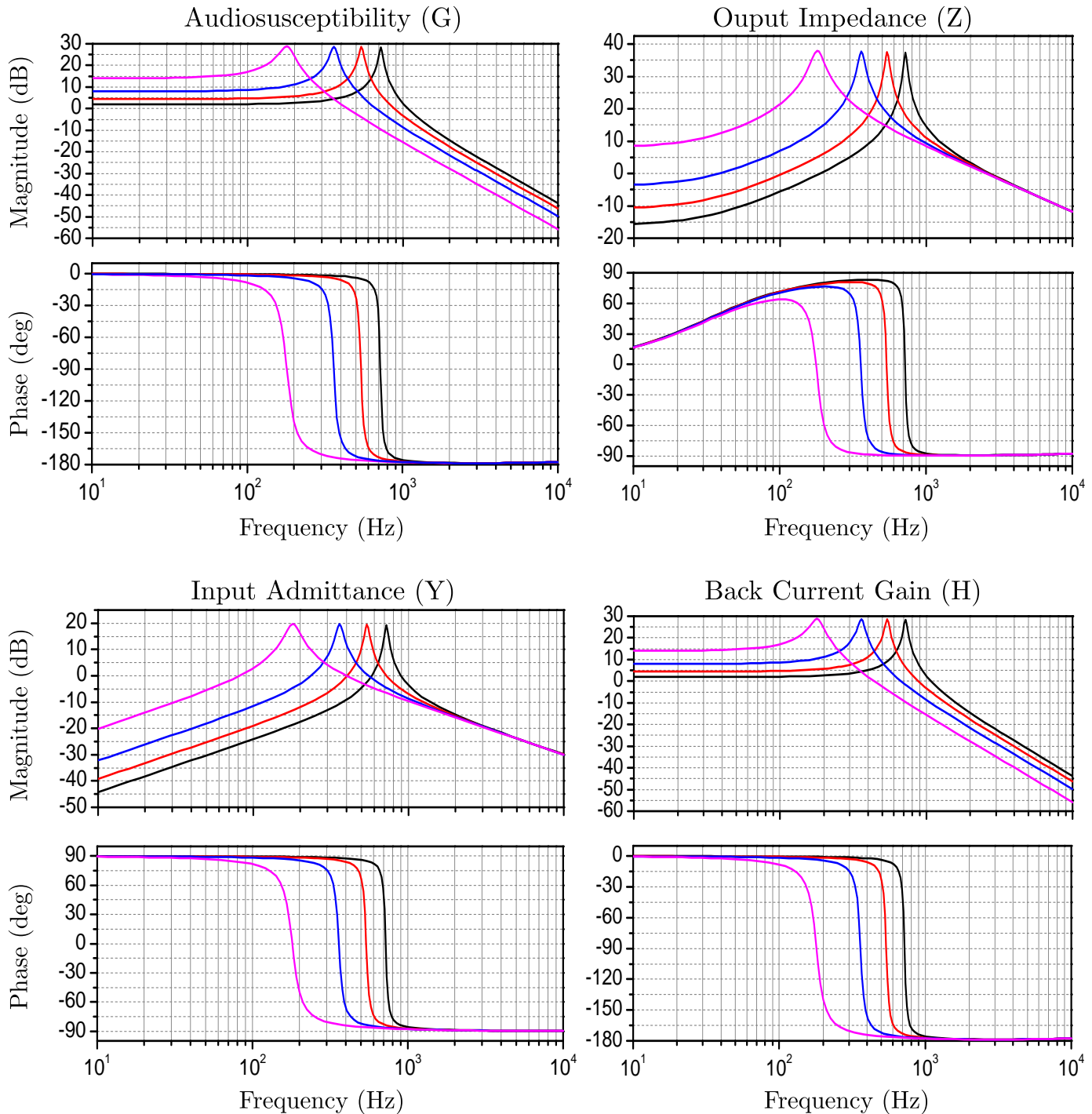

$-\mathrm{d}=0.2$

$-\mathrm{d}=0.4$

$-\mathrm{d}=0.6$

$-\mathrm{d}=0.8$

Figure B.4: Variation of the frequency response of the G-parameters of the synchronous boost converter for different values of the duty cycle.

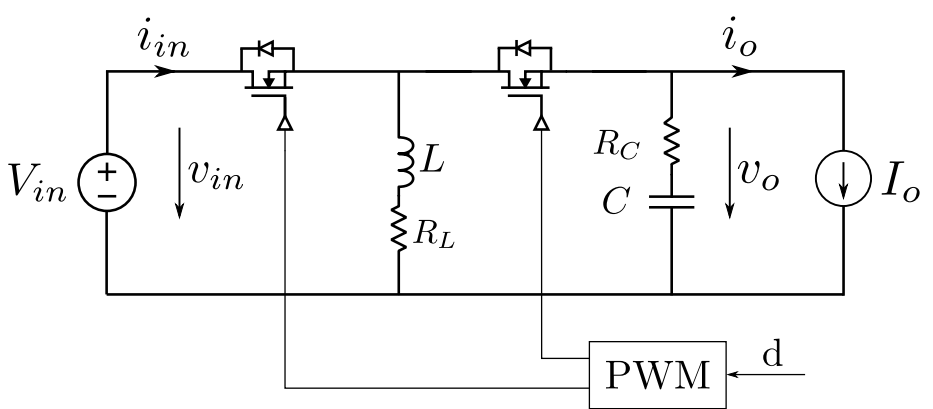

Figure B.5: Synchronous buck-boost converter circuit. 


$$
\operatorname{det}(s I-A)=s^{2}+\frac{R_{L}+(1-D) R_{c}}{L} s+\frac{(1-D)^{2}}{L C}
$$

The zeros of the G-parameters are given by (B.8), where:

$$
\operatorname{adj}(s I-A)=\left(\begin{array}{cc}
s+\frac{R_{L}+(1-D) R_{c}}{L} & \frac{-(1-D)}{C} \\
\frac{1-D}{L} & s
\end{array}\right)
$$

Substituting (B.21) and (B.20) in (B.8), the zeros of the G-parameters can be expressed as:

$$
\begin{aligned}
& \left(\begin{array}{cc}
1 & -(1-D) R_{c} \\
0 & D
\end{array}\right)\left(\begin{array}{cc}
s+\frac{R_{L}+(1-D) R_{c}}{L} & \frac{-(1-D)}{C} \\
\frac{1-D}{L} & s
\end{array}\right)\left(\begin{array}{cc}
0 & \frac{-1}{C} \\
\frac{D}{L} & \frac{-(1-D) R_{c}}{L}
\end{array}\right)+ \\
& +\left(\begin{array}{cc}
0 & -R_{c} \\
0 & 0
\end{array}\right)\left(s^{2}+\frac{R_{L}+(1-D) R_{c}}{L} s+\frac{(1-D)^{2}}{L C}\right)
\end{aligned}
$$

Finally, evaluating (B.22) and (B.20) in (B.5) the transfer functions of the G-parameters can be expressed as:

$$
\begin{aligned}
& G(s)=\frac{-D(1-D)}{L C} \frac{R_{c} C s+1}{s^{2}+\frac{R_{L}+(1-D) R_{c}}{L} s+\frac{(1-D)^{2}}{L C}} \\
& Z(s)=\frac{R_{c} s^{2}+\frac{L+R_{c} C\left(R_{L}+R_{c}(1-D) D\right)}{L C} s+\frac{R_{L}+R_{c}(1-D) D}{L C}}{s^{2}+\frac{R_{L}+(1-D) R_{c}}{L} s+\frac{(1-D)^{2}}{L C}} \\
& Y(s)=\frac{D^{2}}{L} \frac{s^{2}+\frac{R_{L}+(1-D) R_{c}}{s} s+\frac{(1-D)^{2}}{L C}}{L} \\
& H(s)=\frac{-D(1-D)}{L C} \frac{R_{c} C s+1}{s^{2}+\frac{R_{L}+(1-D) R_{c}}{L} s+\frac{(1-D)^{2}}{L C}}
\end{aligned}
$$

The natural frequency, $\omega_{n}$, and the damping coefficient, $\xi$, can be expressed as: 

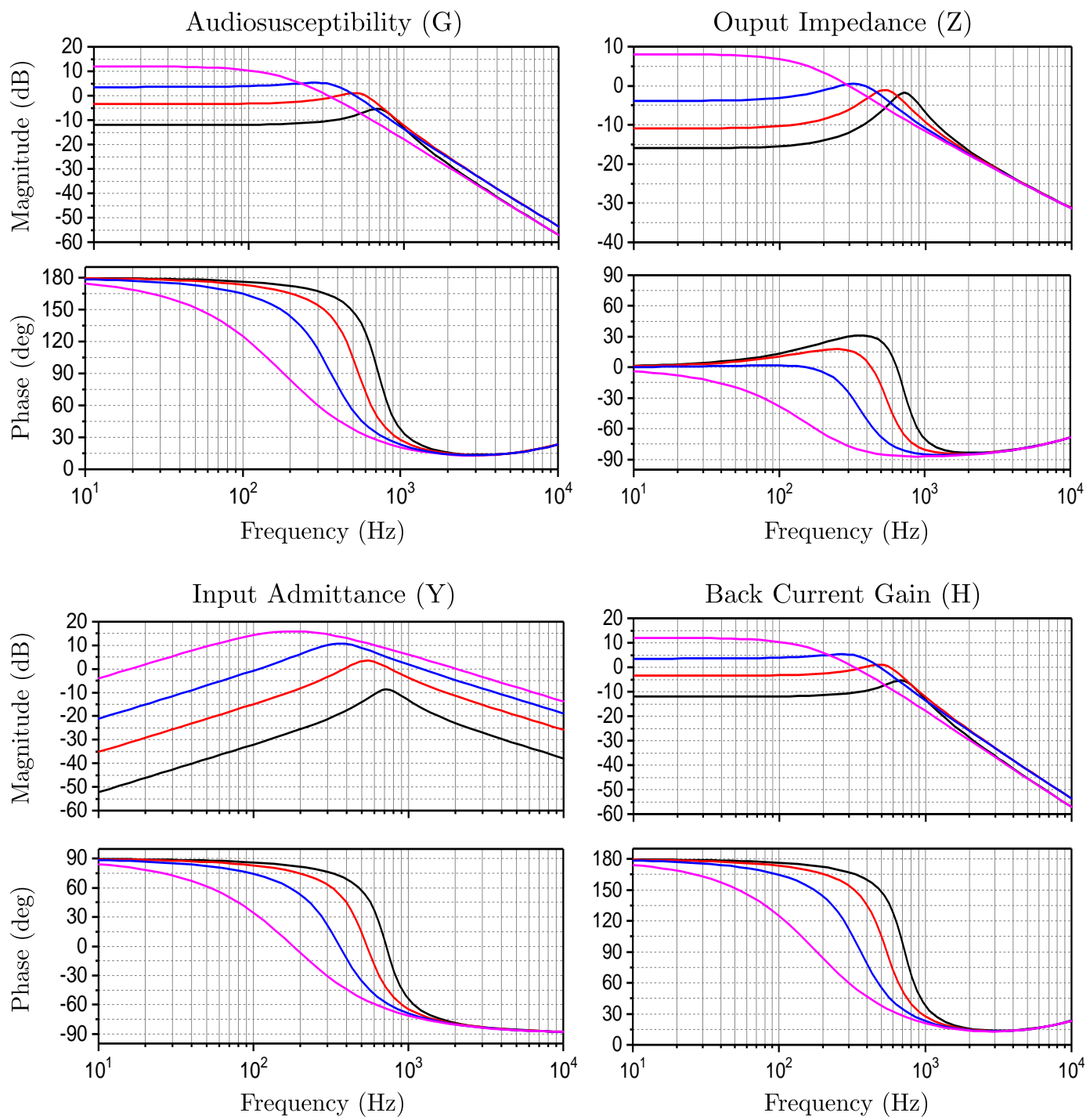

$-\mathrm{d}=0.2$

$-\mathrm{d}=0.4$

$-\mathrm{d}=0.6$

$-\mathrm{d}=0.8$

Figure B.6: Variation of the frequency response of the G-parameters of the synchronous buck-boost converter for different values of the duty cycle.

$$
\begin{aligned}
\omega_{n} & =\frac{1-D}{\sqrt{L C}} \\
\xi & =\frac{1}{2}\left(\frac{R_{L}}{1-D}+R_{c}\right) \sqrt{\frac{C}{L}}
\end{aligned}
$$

In Fig. B.6 the frequency response of the G-parameters for different values of the duty cycle is presented. The parameters evaluated are the ones specified in Fig. 3.16. 



\section{State-space representation of regulated converters}

\section{C.1 Synchronous buck converter}

In this section the state space representation of the voltage-controlled buck converter shown in Fig. C.1 is derived. The state space representation of this converter in open loop including the effect of the duty cycle is defined as:

$$
\begin{aligned}
\left(\begin{array}{c}
\frac{d v_{c}}{d t} \\
\frac{d i_{L}}{d t}
\end{array}\right)=\left(\begin{array}{cc}
0 & \bar{C} \\
\frac{-1}{L} & \frac{-\left(R_{L}+R_{c}\right)}{L}
\end{array}\right)\left(\begin{array}{c}
v_{c} \\
i_{L}
\end{array}\right)+\left(\begin{array}{cc}
0 & \frac{-1}{C} \\
\frac{D}{L} & \frac{R_{c}}{L}
\end{array}\right)\left(\begin{array}{c}
v_{i n} \\
i_{o}
\end{array}\right)+\left(\begin{array}{c}
0 \\
V i n \\
\frac{V i n}{L}
\end{array}\right) d \\
\left(\begin{array}{c}
v_{o} \\
i_{\text {in }}
\end{array}\right)=\left(\begin{array}{cc}
1 & R_{c} \\
0 & D
\end{array}\right)\left(\begin{array}{c}
v_{c} \\
i_{L}
\end{array}\right)+\left(\begin{array}{cc}
0 & -R_{c} \\
0 & 0
\end{array}\right)\left(\begin{array}{c}
v_{i n} \\
i_{o}
\end{array}\right)+\left(\begin{array}{c}
0 \\
I_{L}
\end{array}\right) d
\end{aligned}
$$

where the variables in uppercase represent the operating point where the equations are linearized, i.e. $D$ and $V_{i n}$. The output of the duty cycle can be defined as the action of the regulator having the error signal as an input:

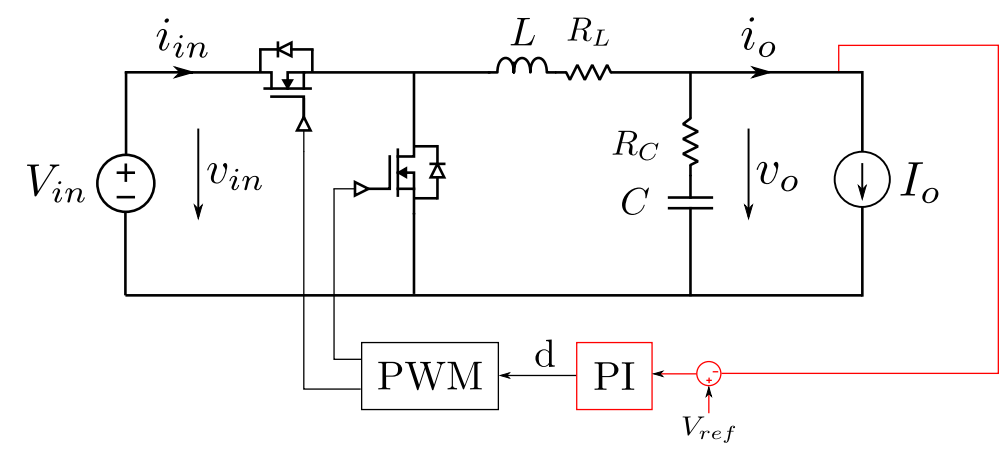

Figure C.1: Voltage-controlled synchronous Buck converter circuit. 


$$
d=K_{p}\left(V_{\text {ref }}-v_{o}\right)+\frac{K_{i}}{s}\left(V_{\text {ref }}-v_{o}\right)
$$

The new integral action inserts a new state variable in the system, so defining a new state variable as:

$$
x_{3}=\frac{K_{i}}{s}\left(V_{\text {ref }}-v_{o}\right)
$$

its derivative can be expressed as:

$$
\frac{d x_{3}}{d t}=K_{i}\left(V_{\text {ref }}-v_{o}\right)
$$

The duty cycle can be expressed as a function of the new state variable:

$$
d=K_{p}\left(V_{\text {ref }}-v_{o}\right)+x_{3}
$$

Finally, including (C.5) in (C.1) and rearranging terms, the state space representation of the voltage-controlled buck converter is obtained as a function of its parameters and the operating point where it is linearized:

$$
\begin{aligned}
& \left(\begin{array}{c}
\frac{d v_{c}}{d t} \\
\frac{d i_{L}}{d t} \\
\frac{d x_{3}}{d t}
\end{array}\right)=\left(\begin{array}{ccc}
0 & \frac{1}{C} & 0 \\
\frac{-1-K_{p} V_{i n}}{L} & \frac{-\left(R_{L}+R_{c}\right)-K_{p} V_{i n} R_{c}}{L} & \frac{V_{i n}}{L} \\
-K_{i} & -K_{i} R_{c} & 0
\end{array}\right)\left(\begin{array}{c}
v_{c} \\
i_{L} \\
x_{3}
\end{array}\right)+ \\
& +\left(\begin{array}{cc}
0 & \frac{-1}{C} \\
\frac{D}{L} & \frac{R_{c}+K_{p} V_{i n} R_{c}}{L} \\
0 & K_{i} R_{c}
\end{array}\right)\left(\begin{array}{c}
v_{i n} \\
i_{o}
\end{array}\right) \\
& \left(\begin{array}{c}
v_{o} \\
i_{i n}
\end{array}\right)=\left(\begin{array}{ccc}
1 & R_{c} & 0 \\
-I_{L} K_{p} & D-I_{L} K_{p} R_{c} & I_{L}
\end{array}\right)\left(\begin{array}{c}
v_{c} \\
i_{L} \\
x_{3}
\end{array}\right)+\left(\begin{array}{cc}
0 & -R_{c} \\
0 & I_{L} K_{p} R_{c}
\end{array}\right)\left(\begin{array}{c}
v_{i n} \\
i_{o}
\end{array}\right)
\end{aligned}
$$

The operating point where the state variables and the duty cycle are linearized can be related with the input variables using inductor volt-second balance and capacitor charge balance:

$$
\begin{aligned}
I_{L} & =I_{o} \\
V_{c} & =V_{o} \\
D & =\frac{V_{c}+\left(R_{L}+R_{c}\right) I_{L}-R_{c} I_{o}}{V_{i n}}=\frac{V_{o}+R_{L} I_{o}}{V_{i n}}
\end{aligned}
$$



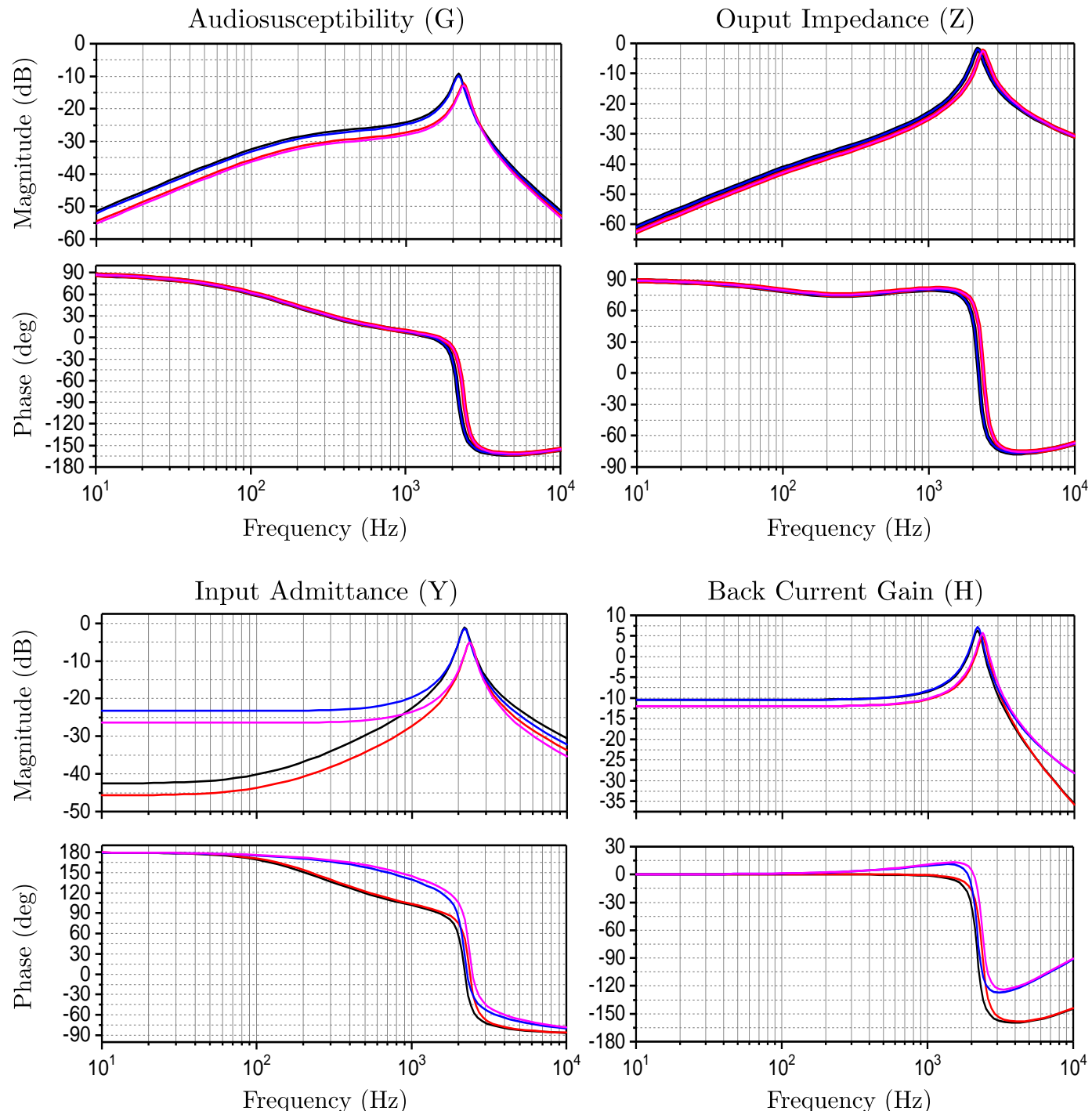

$$
\begin{aligned}
& -V_{i n}=40 \mathrm{~V}, I_{o}=1 \mathrm{~A} \quad-V_{i n}=40 \mathrm{~V}, I_{o}=10 \mathrm{~A} \\
& -V_{i n}=48 \mathrm{~V}, I_{o}=1 \mathrm{~A}-V_{\text {in }}=48 \mathrm{~V}, I_{o}=10 \mathrm{~A}
\end{aligned}
$$

Figure C.2: Frequency response of the G-parameters of the voltage-controlled buck converter.

\section{C.1.1 Evaluation of the equations for different operating points}

The equations derived above are evaluated for different operating points, the parameters considered are the ones presented in Fig. 3.3. The relationship between the state-space representation and the input-output transfer functions shown in B.4 is used to obtain the Gparameters model for the different operating points. The operating points considered are all the possible combinations of $V_{i n}=\{40,48\}$ and $I_{o}=\{1,10\}$. The frequency response of these transfer functions is presented in Fig. C.2. Besides, the parameters of the transfer functions of the G-parameters related with variations in the output current (output impedance and back current gain) are shown in Table. C.1, whereas the parameters of the transfer functions related with variations in the input voltage (audiosusceptibility and input admittance) are presented in Table. C.2. 
Table C.1: Parameters of the transfer functions related to the output current of the voltage-controlled boost converter for different operating points.

\begin{tabular}{|c|c|}
\hline Output impedance, $\mathrm{Z}(\mathrm{s})$ & Back current gain, $\mathrm{H}(\mathrm{s})$ \\
\hline \multicolumn{2}{|c|}{$V_{\text {in }}=40 \mathrm{~V}, I_{o}=1 \mathrm{~A}$} \\
\hline$\frac{0.01 s^{3}+1620 s^{2}+320 e 4 s}{s^{3}+3186.63 s^{2}+191.25 e 6 s+222.05 e 9}$ & $\frac{0.0012 s^{3}+554.55 s^{2}+575.72 e 5 s+666.14 e 8}{s^{3}+3186.63 s^{2}+191.25 e 6 s+222.05 e 9}$ \\
\hline \multicolumn{2}{|c|}{$V_{i n}=48 \mathrm{~V}, I_{o}=1 \mathrm{~A}$} \\
\hline$\frac{0.01 s^{3}+1620 s^{2}+320 e 4 s}{s^{3}+3383.95 s^{2}+223.1 e 6 s+266.46 e 9}$ & $\frac{0.0012 s^{3}+544.63 s^{2}+559.85 e 5 s+666.14 e 8}{s^{3}+3383.95 s^{2}+223.1 e 6 s+266.46 e 9}$ \\
\hline \multicolumn{2}{|c|}{$V_{\text {in }}=40 \mathrm{~V}, I_{o}=10 \mathrm{~A}$} \\
\hline$\frac{0.01 s^{3}+1620 s^{2}+320 e 4 s}{s^{3}+3186.63 s^{2}+191.25 e 6 s+222.05 e 9}$ & $\frac{0.012 s^{3}+2341.59 s^{2}+593.50 e 5 s+666.14 e 8}{s^{3}+3186.63 s^{2}+191.25 e 6 s+222.05 e 9}$ \\
\hline \multicolumn{2}{|c|}{$V_{\text {in }}=48 \mathrm{~V}, I_{o}=10 \mathrm{~A}$} \\
\hline$\frac{0.01 s^{3}+1620 s^{2}+320 e 4 s}{s^{3}+3383.95 s^{2}+223.1 e 6 s+266.46 e 9}$ & $\frac{0.012 s^{3}+2332.43 s^{2}+578.83 e 5 s+666.14 e 8}{s^{3}+3383.95 s^{2}+223.1 e 6 s+266.46 e 9}$ \\
\hline
\end{tabular}

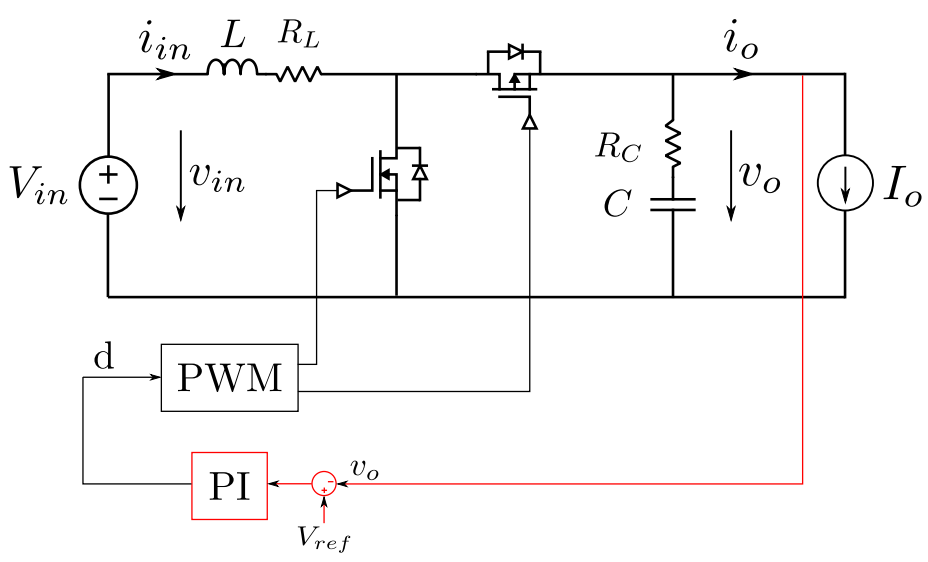

Figure C.3: Voltage-controlled synchronous Boost converter circuit.

\section{C.2 Synchronous boost converter}

In this section the state space representation of the voltage-controlled boost converter shown in Fig. C.3 is derived. The state space representation of this converter in open loop including the effect of the duty cycle is defined as: 
Table C.2: Parameters of the transfer functions related to the input voltage of the voltage-controlled boost converter for different operating points.

\begin{tabular}{|c|c|}
\hline Audiosusceptibility, G(s) & Input admittance, $Y(s)$ \\
\hline \multicolumn{2}{|c|}{$V_{i n}=40 \mathrm{~V}, I_{o}=1 \mathrm{~A}$} \\
\hline$\frac{59.5 s^{2}+952 e 4 s-5.3 e-10}{s^{3}+318663 s^{2}+19125 e 6 s+22205 e 9}$ & $\frac{1762.79 s^{2}-118.4410 e 4 s-165.15 e 7}{s^{3}+318663 s^{2}+19125 e 6 s+22205 e 0}$ \\
\hline \multicolumn{2}{|c|}{$V_{\text {in }}=48 \mathrm{~V}, I_{o}=1 \mathrm{~A}$} \\
\hline$\frac{49.58 s^{2}+793.33 e 4 s-4.4 e-10}{s^{3}+3383.95 s^{2}+223.1 e 6 s+266.46 e 9}$ & $\frac{1223.14 s^{2}-987.01 e 3 s-137.62 e 7}{s^{3}+3383.95 s^{2}+223.1 e 6 s+266.46 e 9}$ \\
\hline \multicolumn{2}{|c|}{$V_{\text {in }}=40 \mathrm{~V}, I_{o}=10 \mathrm{~A}$} \\
\hline$\frac{55 s^{2}+880 e 4 s-4.9 e-10}{s^{3}+3186.63 s^{2}+191.25 e 6 s+222.05 e 9}$ & $\frac{1444.67 s^{2}-109.48 e 5 s-152.66 e 8}{s^{3}+3186.63 s^{2}+191.25 e 6 s+222.05 e 9}$ \\
\hline \multicolumn{2}{|c|}{$V_{i n}=48 \mathrm{~V}, I_{o}=10 \mathrm{~A}$} \\
\hline$\frac{45.83 s^{2}+733.33 e 4 s-4.1 e-10}{s^{3}+3383.95 s^{2}+223.1 e 6 s+266.46 e 9}$ & $\frac{993.82 s^{2}-912.36 e 4 s-127.21 e 8}{s^{3}+3383.95 s^{2}+223.1 e 6 s+266.46 e 9}$ \\
\hline
\end{tabular}

$$
\begin{aligned}
\left(\begin{array}{c}
\frac{d v_{c}}{d t} \\
\frac{d i_{L}}{d t}
\end{array}\right) & =\left(\begin{array}{cc}
0 & \frac{1-D}{C} \\
\frac{-(1-D)}{L} & \frac{-\left(R_{L}+(1-D) R_{c}\right)}{L}
\end{array}\right)\left(\begin{array}{c}
v_{c} \\
i_{L}
\end{array}\right)+\left(\begin{array}{cc}
0 & \frac{-1}{C} \\
\frac{1}{L} & \frac{(1-D) R_{c}}{L}
\end{array}\right)\left(\begin{array}{c}
v_{i n} \\
i_{o}
\end{array}\right)+ \\
& +\left(\begin{array}{c}
\frac{-I_{L}}{C} \\
\frac{V_{c}+R_{c}\left(I_{L}-I_{o}\right)}{L}
\end{array}\right) d \\
\left(\begin{array}{c}
v_{o} \\
i_{\text {in }}
\end{array}\right) & =\left(\begin{array}{cc}
1 & (1-D) R_{c} \\
0 & 1
\end{array}\right)\left(\begin{array}{c}
v_{c} \\
i_{L}
\end{array}\right)+\left(\begin{array}{cc}
0 & -R_{c} \\
0 & 0
\end{array}\right)\left(\begin{array}{c}
v_{i n} \\
i_{o}
\end{array}\right)+\left(\begin{array}{c}
-I_{L} R_{c} \\
0
\end{array}\right) d
\end{aligned}
$$

where the variables in uppercase represent the operating point where the equations are linearized, i.e. $D, I_{L}, I_{O}$ and $V_{c}$. The output of the duty cycle can be defined as the action of the regulator having the error signal as an input, as shown in (C.2). As in the buck case, a new state variable appears in the system, which is defined as (C.3), which has a derivative (C.4). The duty cycle can be expressed as a function of the state variables and the inputs of the system (C.5). Finally, evaluating (C.5) in (C.1) and rearranging terms, 
the state space representation of the voltage controlled boost converter can be expressed as a function of its parameters and the operating point where it is linearized:

$$
\begin{aligned}
& \left(\begin{array}{c}
\frac{d v_{c}}{d t} \\
\frac{d i_{L}}{d t} \\
\frac{d x_{3}}{d t}
\end{array}\right)=\left(\begin{array}{ccc}
\frac{I_{L} K_{p}}{M C} & \frac{1-D}{M C} & \frac{-I_{L}}{M C} \\
\frac{-(1-D) M-F K_{p}}{M L} & \frac{-\left(R_{L}+(1-D) R_{c}\right) M-F K_{p} R_{c}(1-D)}{M L} & \frac{F}{M L} \\
\frac{-K_{i}}{M} & \frac{-(1-D) K_{i} R_{c}}{M} & \frac{I_{L} K_{i} R_{c}}{M}
\end{array}\right)\left(\begin{array}{c}
v_{c} \\
i_{L} \\
x_{3}
\end{array}\right) \\
& +\left(\begin{array}{cc}
0 & \frac{-1}{M C} \\
\frac{1}{L} & \frac{(1-D) R_{c} M-F K_{p} R_{c}}{M L} \\
0 & \frac{K_{i} R_{c}}{M}
\end{array}\right)\left(\begin{array}{c}
v_{i n} \\
i_{o}
\end{array}\right) \\
& \left(\begin{array}{c}
v_{o} \\
i_{\text {in }}
\end{array}\right)=\left(\begin{array}{ccc}
\frac{1}{M} & \frac{(1-D) R_{c}}{M} & \frac{-I_{L} R_{c}}{M} \\
0 & 1 & 0
\end{array}\right)\left(\begin{array}{c}
v_{c} \\
i_{L} \\
x_{3}
\end{array}\right)+\left(\begin{array}{cc}
0 & \frac{-R_{c}}{M} \\
0 & 0
\end{array}\right)\left(\begin{array}{c}
v_{i n} \\
i_{o}
\end{array}\right)
\end{aligned}
$$

where

$$
\begin{aligned}
M & =1-I_{L} R_{c} K_{p} \\
F & =V_{c}+R_{c} I_{L}-I_{o} R_{c}
\end{aligned}
$$

The operating point where the state variables and the duty cycle are linearized can be related with the input variables using inductor volt-second balance and capacitor charge balance:

$$
\begin{aligned}
& V_{c}=V_{\text {ref }} \\
& I_{L}=\frac{-1+\sqrt{1+\frac{4 R_{L} V_{c} I_{o}}{V_{i n}^{2}}}}{2 R_{L} V_{\text {in }}} \\
& D=1-\frac{V_{\text {in }}-I_{L} R_{L}}{V_{c}}
\end{aligned}
$$

\section{C.2.1 Evaluation of the equations for different operating points}

The equations derived above are evaluated for different operating points, the parameters considered are the ones presented in Fig. 3.13. The relationship between the state-space 

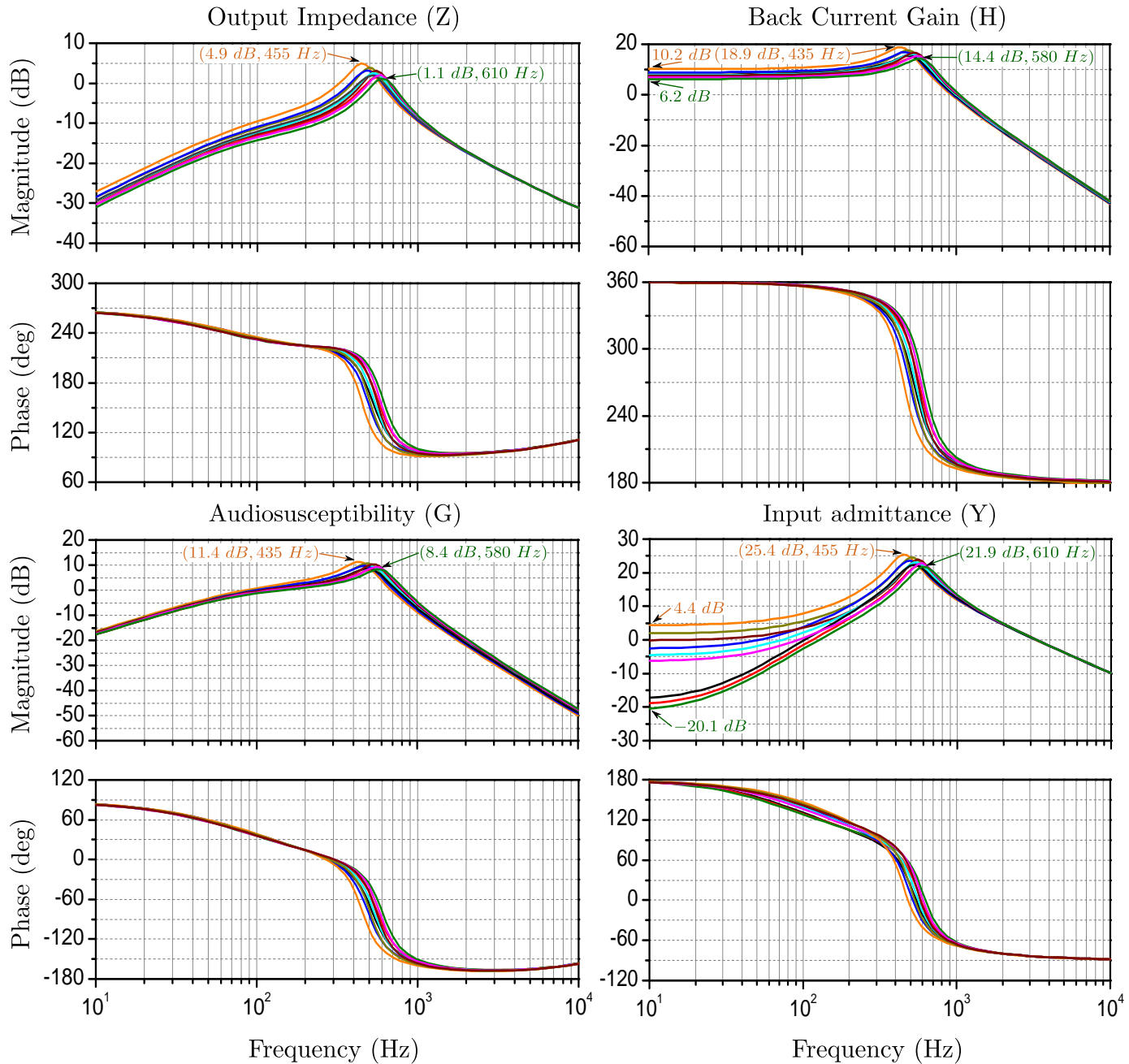

$$
\begin{aligned}
& -V_{i n}=20 V, I_{o}=1 \mathrm{~A}-V_{i n}=20 \mathrm{~V}, I_{o}=5 \mathrm{~A}-V_{i n}=20 \mathrm{~V}, I_{o}=9 \mathrm{~A} \\
& -V_{i n}=22 \mathrm{~V}, I_{o}=1 \mathrm{~A}-V_{\text {in }}=22 \mathrm{~V}, I_{o}=5 \mathrm{~A}-V_{i n}=22 \mathrm{~V}, I_{o}=9 \mathrm{~A} \\
& -V_{i n}=24 \mathrm{~V}, I_{o}=1 \mathrm{~A}-V_{i n}=24 \mathrm{~V}, I_{o}=5 \mathrm{~A}-V_{i n}=24 \mathrm{~V}, I_{o}=9 \mathrm{~A}
\end{aligned}
$$

Figure C.4: Frequency response of the G-parameters of the voltage-controlled boost converter.

representation and the input-output transfer functions shown in B.4 is used to obtain the G-parameters model for the different operating points. The operating points considered are all the possible combinations of $V_{i n}=\{20,22,24\}$ and $I_{o}=\{1,5,9\}$. The frequency response of these transfer functions is presented in Fig. C.4. Besides, the parameters of the transfer functions of the G-parameters related with variations in the output current (output impedance and back current gain) are shown in Table. C.3, whereas the parameters of the transfer functions related with variations in the input voltage (audiosusceptibility and input admittance) are presented in Table. C.4.

\section{C.3 Buck-boost converter}

In this section the state space representation of the voltage-controlled buck-boost converter shown in Fig. C.5 is derived. The state space representation of this converter in open loop including the effect of the duty cycle is defined as: 
Table C.3: Parameters of the transfer functions related to the output current of the voltage-controlled boost converter for different operating points.

\begin{tabular}{|c|c|}
\hline Output impedance, Z(s) & Back current gain, H(s) \\
\hline \multicolumn{2}{|c|}{$V_{i n}=20 \mathrm{~V}, I_{o}=1 \mathrm{~A}$} \\
\hline $0.01 s^{3}+1.621 e 3 s^{2}+340.48 e 4 s+3.43 e-26$ & $-13.73 s^{2}+286.43 e 5 s+153.72 e 8$ \\
\hline$s^{3}+2082.72 s^{2}+116.67 e 5 s+624.47 e 7$ & $s^{3}+2082.72 s^{2}+116.67 e 5 s+624.47 e 7$ \\
\hline \multicolumn{2}{|c|}{$V_{i n}=22 \mathrm{~V}, I_{o}=1 \mathrm{~A}$} \\
\hline $0.01 s^{3}+1621.72 s^{2}+341.95 e 4 s+1 e-8$ & $-5.3 s^{2}+299.9 e 5 s+153.71 e 8$ \\
\hline$s^{3}+2098.83 s^{2}+134.96 e 5 s+689.9 e 7$ & $\overline{s^{3}+2098.83 s^{2}+134.96 e 5 s+689.9 e 7}$ \\
\hline \multicolumn{2}{|c|}{$V_{i n}=24 \mathrm{~V}, I_{o}=1 \mathrm{~A}$} \\
\hline $0.01 s^{3}-1621.78 s^{2}+343.31 e 4 s+2.1 e-8$ & $3.12 s^{2}+313.34 e 5 s+153.69 e 8$ \\
\hline$s^{3}+2114.29 s^{2}+154.33 e 5 s+755.08 e 7$ & $s^{3}+2114.29 s^{2}+154.33 e 5 s+755.08 e 7$ \\
\hline \multicolumn{2}{|c|}{$V_{i n}=20 \mathrm{~V}, I_{o}=5 \mathrm{~A}$} \\
\hline $0.01 s^{3}+1623.31 s^{2}+340.06 e 4 s+1.1 e-8$ & $-18.3 s^{2}+280.18 e 5 s+154.28 e 8$ \\
\hline$s^{3}+1908.46 s^{2}+102.79 e 5 s+557.89 e 7$ & $s^{3}+1908.46 s^{2}+102.79 e 5 s+557.89 e 7$ \\
\hline \multicolumn{2}{|c|}{$V_{i n}=22 \mathrm{~V}, I_{o}=5 \mathrm{~A}$} \\
\hline $0.01 s^{3}+1623.2 s^{2}+341.63 e 4 s+1.5 e-6$ & $-9,38 s^{2}+294.29 e 5 s+154.18 e 8$ \\
\hline$s^{3}+1942.85 s^{2}+122 e 5 s+630.25 e 7$ & $\overline{s^{3}+1942.85 s^{2}+122 e 5 s+630.25 e 7}$ \\
\hline \multicolumn{2}{|c|}{$V_{i n}=24 \mathrm{~V}, I_{o}=5 \mathrm{~A}$} \\
\hline $0.01 s^{3}+1623.12 s^{2}+343.07 e 4 s+1.1 e-8$ & $-0.57 s^{2}+308.24 e 5 s+154.1 e 8$ \\
\hline$s^{3}+1972.93 s^{2}+142.11 e 5 s+700.97 e 7$ & $\overline{s^{3}+1972.93 s^{2}+142.11 e 5 s+700.97 e 7}$ \\
\hline \multicolumn{2}{|c|}{$V_{i n}=20 \mathrm{~V}, I_{o}=9 \mathrm{~A}$} \\
\hline $0.01 s^{3}+1625.19 s^{2}+339.54 e 4 s+1.5-9$ & $-23.52 s^{2}+273.07 e 5 s+154.96 e 8$ \\
\hline$s^{3}+1709.18 s^{2}+872.96 e 4 s+481.88 e 7$ & $s^{3}+1709.18 s^{2}+872.96 e 4 s+481.88 e 7$ \\
\hline \multicolumn{2}{|c|}{$V_{i n}=22 \mathrm{~V}, I_{o}=9 \mathrm{~A}$} \\
\hline $0.01 s^{3}+1624.83 s^{2}+341.25 e 4 s+1.1 e-8$ & $-13.91 s^{2}+288.08 e 5 s+154.74 e 8$ \\
\hline$s^{3}+1769.64 s^{2}+107.91 e 5 s+564.13 e 7$ & $s^{3}+1769.64 s^{2}+107.91 e 5 s+564.13 e 7$ \\
\hline \multicolumn{2}{|c|}{$V_{\text {in }}=24 V, I_{o}=9 \mathrm{~A}$} \\
\hline $0.01 s^{3}+1624.57 s^{2}+342.78 e 4 s$ & $-4.59 s^{2}+302.72 e 5 s+154.56 e 8$ \\
\hline$\overline{s^{3}+1819.1 s^{2}+129.04 e 5 s+642.19 e 7}$ & $\overline{s^{3}+1819.1 s^{2}+129.04 e 5 s+642.19 e 7}$ \\
\hline
\end{tabular}


Table C.4: Parameters of the transfer functions related to the input voltage of the voltage-controlled boost converter for different operating points.

\begin{tabular}{|c|c|}
\hline Audiosusceptibility, G(s) & Input admittance, $Y(s)$ \\
\hline \multicolumn{2}{|c|}{$V_{i n}=20 \mathrm{~V}, I_{o}=1 \mathrm{~A}$} \\
\hline $82.34 s^{2}+131.74 e 5 s+6.5 e-10$ & $2 e 4 s^{2}-782.73 e 3 s-777.87 e 6$ \\
\hline$s^{3}+2082.72 s^{2}+116.67 e 5 s+624.47 e 7$ & $s^{3}+2082.72 s^{2}+116.67 e 5 s+624.47 e 7$ \\
\hline \multicolumn{2}{|c|}{$V_{i n}=22 \mathrm{~V}, I_{o}=1 \mathrm{~A}$} \\
\hline $90.77 s^{2}+145.23 e 5 s+2.8 e-10$ & $2 e 4 s^{2}-709.99 e 3 s-705.58 e 6$ \\
\hline$\overline{s^{3}+2098.83 s^{2}+134.96 e 5 s+689.9 e 7}$ & $\overline{s^{3}+2098.83 s^{2}+134.96 e 5 s+689.9 e 7}$ \\
\hline \multicolumn{2}{|c|}{$V_{i n}=24 V, I_{o}=1 \mathrm{~A}$} \\
\hline $99.18 s^{2}+158.69 e 5 s+3 e-10$ & $2 e 4 s^{2}-649.73 e 3 s-645.69 e 6$ \\
\hline$s^{3}+2114.29 s^{2}+154.33 e 5 s+755.08 e 7$ & $s^{3}+2114.29 s^{2}+154.33 e 5 s+755.08 e 7$ \\
\hline \multicolumn{2}{|c|}{$V_{i n}=20 \mathrm{~V}, I_{o}=5 \mathrm{~A}$} \\
\hline $78.1 s^{2}+124.93 e 5 s-5.5 e-9$ & $2 e 4 s^{2}-414.12 e 4 s-411.55 e 7$ \\
\hline$s^{3}+1908.46 s^{2}+102.79 e 5 s+557.89 e 7$ & $s^{3}+1908.46 s^{2}+102.79 e 5 s+557.89 e 7$ \\
\hline \multicolumn{2}{|c|}{$V_{i n}=22 V, I_{o}=5 A$} \\
\hline $86.96 s^{2}+139.14 e 5 s+3.1 e-9$ & $2 e 4 s^{2}-371.63 e 4 s-369.32 e 7$ \\
\hline$\overline{s^{3}+1942.85 s^{2}+122 e 5 s+630.25 e 7}$ & $\overline{s^{3}+1942.85 s^{2}+122 e 5 s+630.25 e 7}$ \\
\hline \multicolumn{2}{|c|}{$V_{i n}=24 \mathrm{~V}, I_{o}=5 \mathrm{~A}$} \\
\hline $95.74 s^{2}+153.18 e 5 s+3.4 e-9$ & $2 e 4 s^{2}-337.42 e 4 s-335.33 e 7$ \\
\hline$\overline{s^{3}+1972.93 s^{2}+142.11 e 5 s+700.97 e 7}$ & $\overline{s^{3}+1972.93 s^{2}+142.11 e 5 s+700.97 e 7}$ \\
\hline \multicolumn{2}{|c|}{$V_{i n}=20 \mathrm{~V}, I_{o}=9 \mathrm{~A}$} \\
\hline $73.21 s^{2}+117.14 e 5 s-5.2 e-9$ & $2 e 4 s^{2}-798.22 e 4 s-793.26 e 7$ \\
\hline$s^{3}+1709.18 s^{2}+872.96 e 4 s+481.88 e 7$ & $s^{3}+1709.18 s^{2}+872.96 e 4 s+481.88 e 7$ \\
\hline \multicolumn{2}{|c|}{$V_{i n}=22 \mathrm{~V}, I_{o}=9 \mathrm{~A}$} \\
\hline $82.74 s^{2}+132.38 e 5 s-1 e-24$ & $2 e 4 s^{2}-705.48 e 4 s-701.1 e 7$ \\
\hline$s^{3}+1769.64 s^{2}+107.91 e 5 s+564.13 e 7$ & $s^{3}+1769.64 s^{2}+107.91 e 5 s+564.13 e 7$ \\
\hline \multicolumn{2}{|c|}{$V_{i n}=24 \mathrm{~V}, I_{o}=9 \mathrm{~A}$} \\
\hline $91.99 s^{2}+147.19 e 5 s$ & $2 e 4 s^{2}-633.93 e 4 s-629.99 e 7$ \\
\hline$\overline{s^{3}+1819.1 s^{2}+129.04 e 5 s+642.19 e 7}$ & $s^{3}+1819.1 s^{2}+129.04 e 5 s+642.19 e 7$ \\
\hline
\end{tabular}




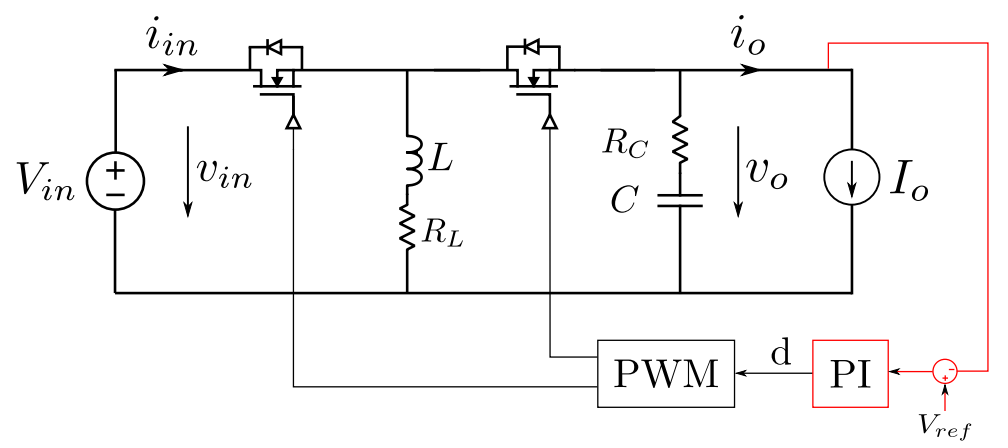

Figure C.5: Voltage-controlled synchronous Buck-boost converter circuit.

$$
\begin{aligned}
\left(\begin{array}{c}
\frac{d v_{c}}{d t} \\
\frac{d i_{L}}{d t}
\end{array}\right) & \left(\begin{array}{cc}
0 & \frac{-(1-D)}{C} \\
\frac{(1-D)}{L} & \frac{-\left(R_{L}+(1-D) R_{c}\right)}{L}
\end{array}\right)\left(\begin{array}{c}
v_{c} \\
i_{L}
\end{array}\right)+\left(\begin{array}{cc}
0 & \frac{-1}{C} \\
\frac{D}{L} & \frac{-(1-D) R_{c}}{L}
\end{array}\right)\left(\begin{array}{c}
v_{i n} \\
i_{o}
\end{array}\right)+ \\
& +\left(\begin{array}{c}
\frac{I_{L}}{C} \\
\frac{-V_{c}+V_{i n}+R_{c}\left(I_{o}+I_{L}\right)}{L}
\end{array}\right) d \\
\left(\begin{array}{c}
v_{o} \\
i_{\text {in }}
\end{array}\right) & =\left(\begin{array}{cc}
1 & -(1-D) R_{c} \\
0 & D
\end{array}\right)\left(\begin{array}{c}
v_{c} \\
i_{L}
\end{array}\right)+\left(\begin{array}{cc}
0 & -R_{c} \\
0 & 0
\end{array}\right)\left(\begin{array}{c}
v_{i n} \\
i_{o}
\end{array}\right)+\left(\begin{array}{c}
R_{c} I_{L} \\
I_{L}
\end{array}\right) d
\end{aligned}
$$

where the variables in uppercase represent the operating point where the equations are linearized, i.e. $D, I_{L}, I_{o}, V_{c}$, and $V_{i n}$. The output of the duty cycle can be defined as the action of the regulator having the error signal as an input, as shown in (C.2). As in the previous cases, a new state variable appears in the system, which is defined as (C.3), which has a derivative (C.4). The duty cycle can be expressed as a function of the state variables and the inputs of the system (C.5). Finally, evaluating (C.5) in (C.1) and rearranging terms, the state space representation of the voltage controlled boost converter can be expressed as a function of its parameters and the operating point where it is linearized: 


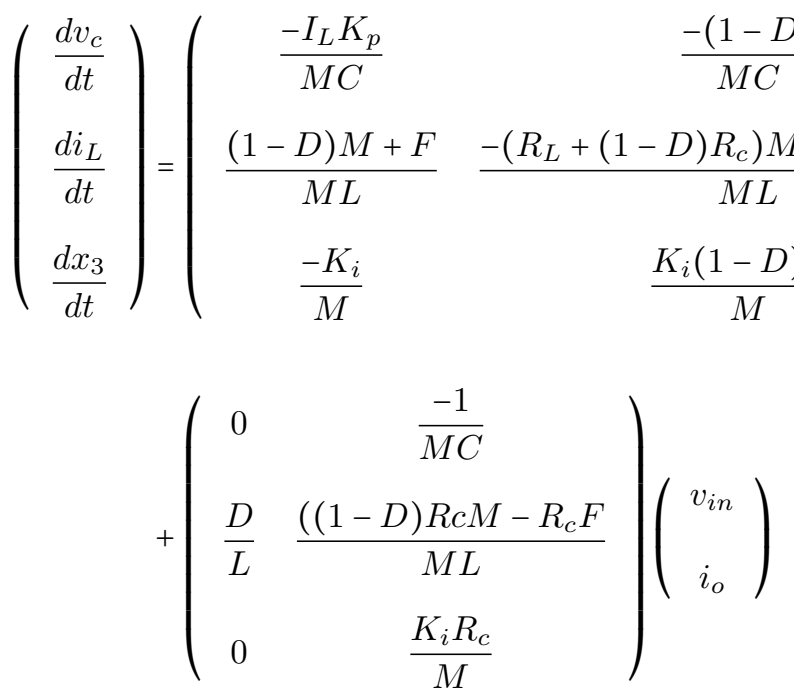

$$
\begin{aligned}
& \left(\begin{array}{c}
v_{o} \\
i_{i n}
\end{array}\right)=\left(\begin{array}{ccc}
\frac{1}{M} & \frac{-(1-D) R_{c}}{M} & \frac{I_{L} R_{c}}{M} \\
\frac{-K_{p} I_{L}}{M} & \frac{D+K_{p} R_{c} I_{L}}{M} & \frac{I_{L}}{M}
\end{array}\right)\left(\begin{array}{c}
v_{c} \\
i_{L} \\
x_{3}
\end{array}\right)+\left(\begin{array}{cc}
0 & \frac{-R_{c}}{M} \\
0 & \frac{K_{p} R_{c} I_{L}}{M}
\end{array}\right)\left(\begin{array}{c}
v_{i n} \\
i_{o}
\end{array}\right)
\end{aligned}
$$

where:

$$
\begin{aligned}
M & =1-I_{L} R_{c} K_{p} \\
F & =-V_{c}+V_{i n}+R_{c} I_{L}-I_{o} R_{c}
\end{aligned}
$$

The operating point where the state variables and the duty cycle are linearized can be related with the input variables using inductor volt-second balance and capacitor charge balance:

$$
\begin{aligned}
& V_{c}=V_{\text {ref }} \\
& I_{L}=\frac{V_{i n}+R_{c} I_{o}}{R_{L}}-\frac{\sqrt{\left(\frac{V_{i n}+R_{c} I_{o}}{R_{L}}\right)^{2}-\frac{4\left(V_{c} I_{o}-R_{c} I_{o}^{2}-V_{i n} I_{o}\right)}{R_{L}}}}{2} \\
& D=\frac{I_{o}+I_{L}}{I_{L}}
\end{aligned}
$$

\section{C.3.1 Evaluation of the equations for different operating points}

The equations derived above are evaluated for different operating points, the parameters considered are the ones presented in Fig. 3.18. The relationship between the state-space representation and the input-output transfer functions shown in B.4 is used to obtain the Gparameters models for the different operating points. The operating points considered are 

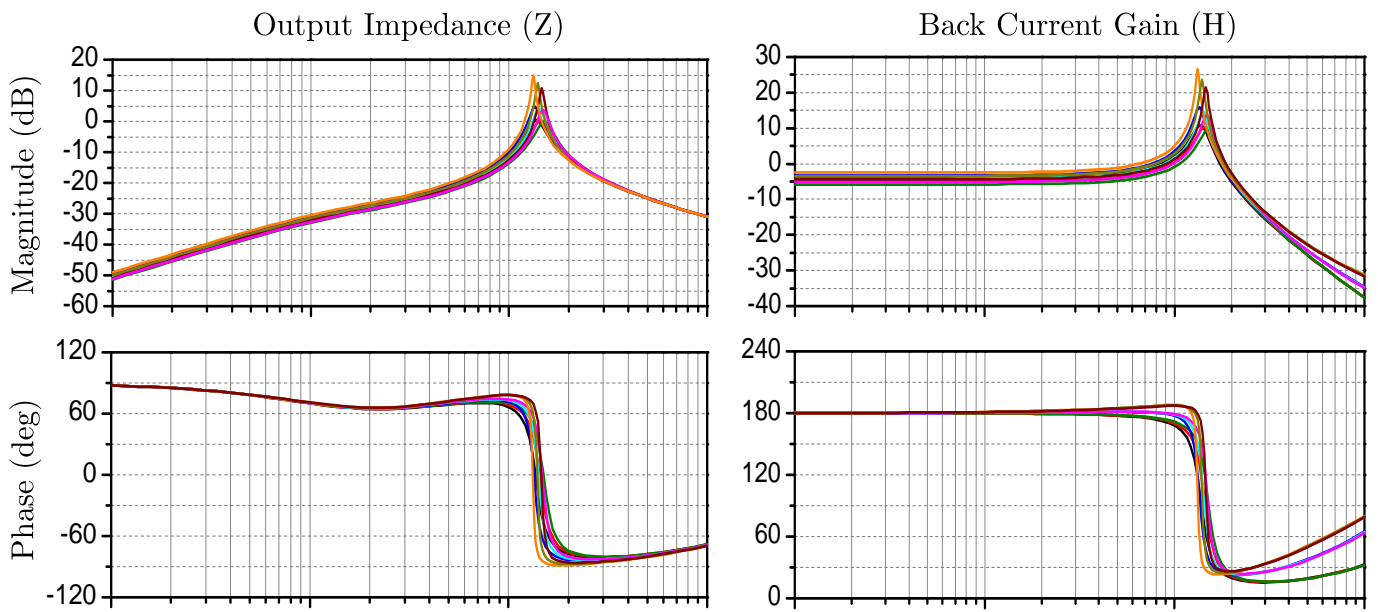

Audiosusceptibility $(\mathrm{G})$

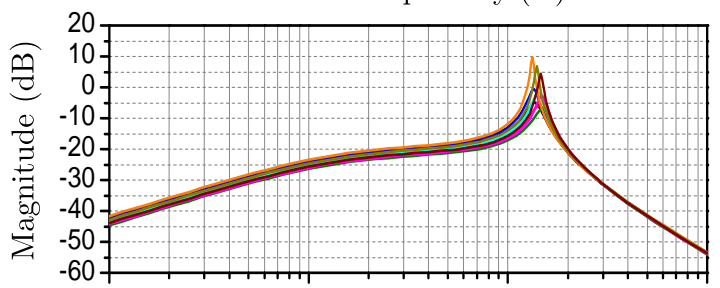

Input admittance (Y)
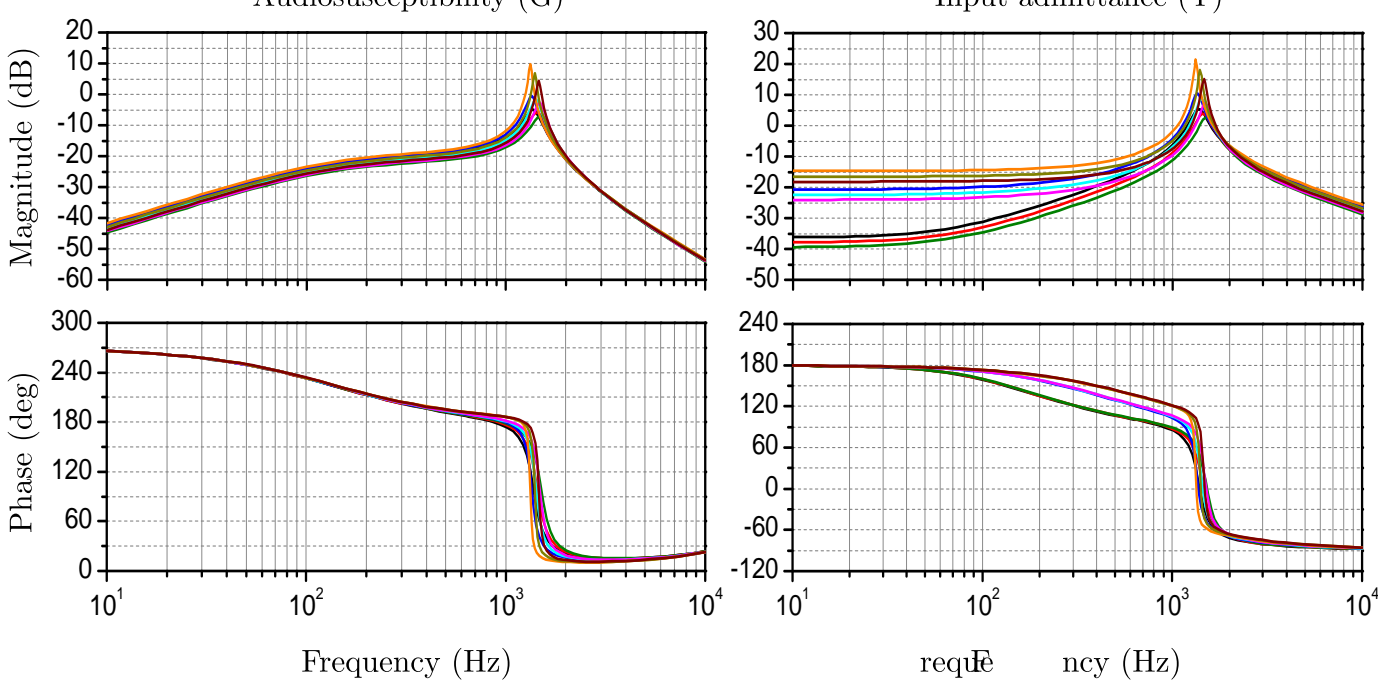

$-V_{i n}=40 \mathrm{~V}, I_{o}=-1 \mathrm{~A} \quad-V_{i n}=40 \mathrm{~V}, I_{o}=-5.5 \mathrm{~A} \quad-V_{i n}=40 \mathrm{~V}, I_{o}=-10 \mathrm{~A}$

$-V_{i n}=44 V, I_{o}=-1 \mathrm{~A} \quad-V_{i n}=44 \mathrm{~V}, I_{o}=-5.5 \mathrm{~A} \quad-V_{i n}=44 \mathrm{~V}, I_{o}=-10 \mathrm{~A}$

$-V_{i n}=48 \mathrm{~V}, I_{o}=-1 \mathrm{~A} \quad-V_{i n}=48 \mathrm{~V}, I_{o}=-5.5 \mathrm{~A} \quad-V_{i n}=48 \mathrm{~V}, I_{o}=-10 \mathrm{~A}$

Figure C.6: Frequency response of the G-parameters of the voltage-controlled buck-boost converter.

all the possible combinations of $V_{i n}=\{40,44,48\}$ and $I_{o}=\{-1,-5.5,-10\}$. The frequency response of these transfer functions is presented in Fig. C.6. Besides, the parameters of the transfer functions of the G-parameters related with variations in the output current (output impedance and back current gain) are shown in Table. C.5, whereas the parameters of the transfer functions related with variations in the input voltage (audiosusceptibility and input admittance) are presented in Table. C.6. 
Table C.5: Parameters of the transfer functions related to the output current of the voltage-controlled buck-boost converter for different operating points.

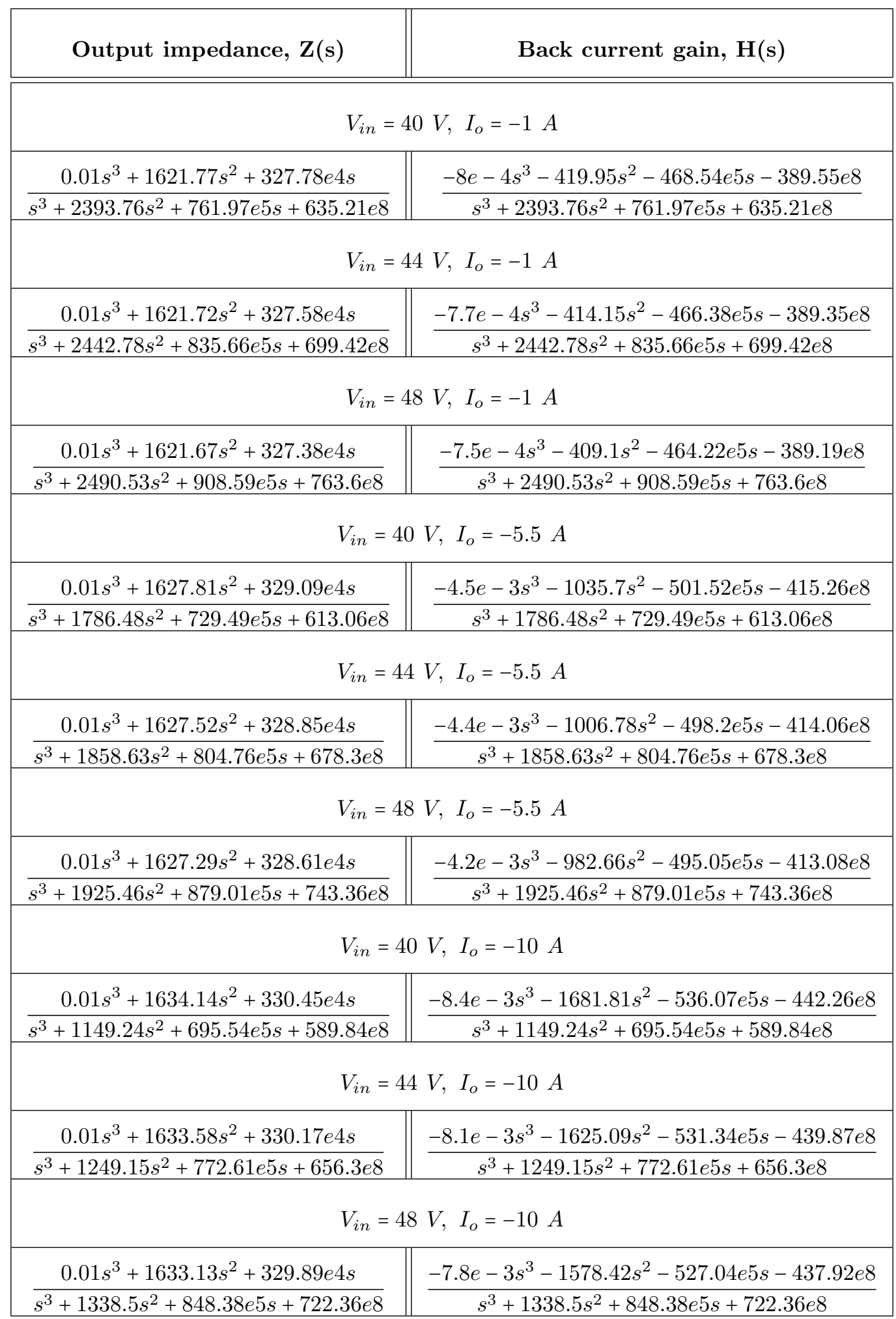


Table C.6: Parameters of the transfer functions related to the input voltage of the voltage-controlled buckboost converter for different operating points.

\begin{tabular}{|c|c|}
\hline Audiosusceptibility, G(s) & Input admittance, $Y(s)$ \\
\hline \multicolumn{2}{|c|}{$V_{\text {in }}=40 \mathrm{~V}, I_{o}=-1 \mathrm{~A}$} \\
\hline$-47.04 s^{2}-752.64 e 4 s-7 e-10$ & $2847,39 s^{2}-977.42 e 3 s-971.35 e 6$ \\
\hline$\overline{s^{3}+2393.76 s^{2}+761.97 e 5 s+635.21 e 8}$ & $\overline{s^{3}+2393.76 s^{2}+761.97 e 5 s+635.21 e 8}$ \\
\hline \multicolumn{2}{|c|}{$V_{\text {in }}=44 \mathrm{~V}, I_{o}=-1 \mathrm{~A}$} \\
\hline$-45.85 s^{2}-733.54 e 4 s+1.3 e-10$ & $2520.84 s^{2}-887.87 e 3 s-882.36 e 6$ \\
\hline$\overline{s^{3}+2442.78 s^{2}+835.66 e 5 s+699.42 e 8}$ & $\bar{s}^{3}+2442.78 s^{2}+835.66 e 5 s+699.42 e 8$ \\
\hline \multicolumn{2}{|c|}{$V_{\text {in }}=48 \mathrm{~V}, I_{o}=-1 \mathrm{~A}$} \\
\hline$-44.62 s^{2}-713.91 e 4 s+2.4 e-9$ & $2247.44 s^{2}-813.38 e 3 s-808.32 e 6$ \\
\hline $\bar{s}^{3}+2490.53 s^{2}+908.59 e 5 s+763.6 e 8$ & $\overline{s^{3}+2490.53 s^{2}+908.59 e 5 s+763.6 e 8}$ \\
\hline \multicolumn{2}{|c|}{$V_{i n}=40 \mathrm{~V}, I_{o}=-5.5 \mathrm{~A}$} \\
\hline$-47.77 s^{2}-764.3 e 4 s+3.2 e-9$ & $3011.28 s^{2}-567.29 e 4 s-563.77 e 7$ \\
\hline$s^{3}+1786.48 s^{2}+729.49 e 5 s+613.06 e 8$ & $s^{3}+1786.48 s^{2}+729.49 e 5 s+613.06 e 8$ \\
\hline \multicolumn{2}{|c|}{$V_{i n}=44 \mathrm{~V}, I_{O}=-5.5 \mathrm{~A}$} \\
\hline$-46.61 s^{2}-745.77 e 4 s+1.3 e-9$ & $2658.82 s^{2}-513.4 e 4 s-510.21 e 7$ \\
\hline$\overline{s^{3}+1858.63 s^{2}+804.76 e 5 s+678.3 e 8}$ & $\overline{s^{3}+1858.63 s^{2}+804.76 e 5 s+678.3 e 8}$ \\
\hline \multicolumn{2}{|c|}{$V_{i n}=48 \mathrm{~V}, I_{o}=-5.5 \mathrm{~A}$} \\
\hline$-45.4 s^{2}-726.43 e 4 s+1.2 e-8$ & $2365.08 s^{2}-468.92 e 4 s-466.01 e 7$ \\
\hline$\overline{s^{3}+1925.46 s^{2}+879.01 e 5 s+743.36 e 8}$ & $s^{3}+1925.46 s^{2}+879.01 e 5 s+743.36 e 8$ \\
\hline \multicolumn{2}{|c|}{$V_{i n}=40 \mathrm{~V}, I_{o}=-10 \mathrm{~A}$} \\
\hline$-48.47 s^{2}-775.59 e 4 s-1.3 e-8$ & $3187.47 s^{2}-109.03 e 5 s-108.35 e 8$ \\
\hline$s^{3}+1149.24 s^{2}+695.54 e 5 s+589.84 e 8$ & $s^{3}+1149.24 s^{2}+695.54 e 5 s+589.84 e 8$ \\
\hline \multicolumn{2}{|c|}{$V_{i n}=44 \mathrm{~V}, I_{o}=-10 \mathrm{~A}$} \\
\hline$-47.36 s^{2}-757.75 e 4 s+1.3 e-8$ & $2806.14 s^{2}-982.59 e 4 s-976.49 e 7$ \\
\hline$s^{3}+1249.15 s^{2}+772.61 e 5 s+656.3 e 8$ & $s^{3}+1249.15 s^{2}+772.61 e 5 s+656.3 e 8$ \\
\hline \multicolumn{2}{|c|}{$V_{i n}=48 \mathrm{~V}, I_{o}=-10 \mathrm{~A}$} \\
\hline$-46.17 s^{2}-738.8 e 4 s$ & $2489.98 s^{2}-894.5 e 4 s-888.95 e 7$ \\
\hline$s^{3}+1338.5 s^{2}+848.38 e 5 s+722$. & $\overline{s^{3}+1338.5 s^{2}+848.38 e 5 s+722.36 e 8}$ \\
\hline
\end{tabular}




\section{Bibliography}

[1] United Nations. (2015) Paris agreement. Accessed 13 Feb. 2018. [Online]. Available: http://unfccc.int/files/essential_background/convention/application/pdf/ english_paris_agreement.pdf.

[2] European Commission. (2015) Energy union - presentation by president juncker. Accessed 07 Feb. 2018. [Online]. Available: http://eur-lex.europa.eu/resource.html? uri=cellar:fa6ea15b-b7b0-11e6-9e3c-01aa75ed71a1.0001.02/DOC_1\&format=PDF.

[3] - (2017) Third report on the state of the energy union. Accessed 06 Feb. 2018. [Online]. Available: https://ec.europa.eu/commission/sites/beta-political/ files/third-report-state-energy-union_en.pdf.

[4] - (2016) Clean energy for all europeans. Accessed 07 Feb. 2018. [Online]. Available: http://eur-lex.europa.eu/resource.html?uri=cellar: fa6ea15b-b7b0-11e6-9e3c-01aa75ed71a1.0001.02/DOC_1\&format=PDF.

[5] - (2006) European smartgrids technology platform. Accessed 07 Feb. 2018. [Online]. Available: https://ec.europa.eu/research/energy/pdf/smartgrids_en.pdf.

[6] P. Palensky and D. Dietrich, "Demand side management: Demand response, intelligent energy systems, and smart loads," IEEE Trans. Ind. Informat., vol. 7, no. 3, pp. 381-388, Aug 2011. IEEE Xplore:

[7] S. Bacha, D. Picault, B. Burger, I. Etxeberria-Otadui, and J. Martins, "Photovoltaics in microgrids: An overview of grid integration and energy management aspects," IEEE Ind. Electron. Mag., vol. 9, no. 1, pp. 33-46, March 2015.

IEEEXP $X$ plore:

[8] Y. Cai, T. Huang, E. Bompard, Y. Cao, and Y. Li, "Self-sustainable community of electricity prosumers in the emerging distribution system," IEEE Trans. Smart Grid, vol. 8, no. 5, pp. 2207-2216, Sept 2017.

IEEEXPlore:

[9] J. Lin, W. Yu, N. Zhang, X. Yang, H. Zhang, and W. Zhao, "A survey on internet of things: Architecture, enabling technologies, security and privacy, and applications," IEEE Internet Things J., vol. 4, no. 5, pp. 1125-1142, Oct 2017.

IEEEXPplore:

[10] R. Morello, C. D. Capua, G. Fulco, and S. C. Mukhopadhyay, "A smart power meter to monitor energy flow in smart grids: The role of advanced sensing and iot in the electric grid of the future," IEEE Sensors J., vol. 17, no. 23, pp. 7828-7837, Dec 2017.

IEEEXPlore: 
[11] L. Li, K. Ota, and M. Dong, "When weather matters: Iotbased electrical load forecasting for smart grid," IEEE Commun. Mag., vol. 55, no. 10, pp. 46-51, OCTOBER 2017.

IEEE $X p_{\text {Dipital library }}$ Q

[12] B. Khan and P. Singh, "Selecting a meta-heuristic technique for smart micro-grid optimization problem: A comprehensive analysis," IEEE Access, vol. 5, pp. 13951-13977, 2017.

IEEE $X p_{\text {Digitullibray }}$ R

[13] "Triumph of ac. 2. the battle of the currents," IEEE Power Energy Mag., vol. 1, no. 4, pp. 70-73, Jul 2003.

IEEE $X p_{\text {Digiallibray }}$ R

[14] P. Fairley, "Dc versus ac: The second war of currents has already begun [in my view]," IEEE Power Energy Mag., vol. 10, no. 6, pp. 104-103, Nov 2012.

IEEE Xplore: Q

[15] J. J. Justo, F. Mwasilu, J. Lee, and J.-W. Jung, "Ac-microgrids versus dc-microgrids with distributed energy resources: A review," Renew. Sust. Energ. Rev., vol. 24, pp. 387 - 405, 2013. [Online]. Available: http://www.sciencedirect.com/science/article/pii/S1364032113002268.

IEEE Xplore: $Q$

[16] T. Dragicevic, J. C. Vasquez, J. M. Guerrero, and D. Skrlec, "Advanced lvdc electrical power architectures and microgrids: A step toward a new generation of power distribution networks." IEEE Electrification Magazine, vol. 2, no. 1, pp. 54-65, March 2014.

IEEE Xplore: Q

[17] D. Fregosi, S. Ravula, D. Brhlik, J. Saussele, S. Frank, E. Bonnema, J. Scheib, and E. Wilson, "A comparative study of dc and ac microgrids in commercial buildings across different climates and operating profiles," in 2015 IEEE First International Conference on DC Microgrids (ICDCM), June 2015, pp. 159-164.

IEEE $X p_{\text {Digital libray }}$ Q

[18] J. M. Guerrero, P. C. Loh, T. L. Lee, and M. Chandorkar, "Advanced control architectures for intelligent microgrids part ii: Power quality, energy storage, and ac/dc microgrids," IEEE Transactions on Industrial Electronics, vol. 60, no. 4, pp. 1263-1270, April 2013.

\section{IEEE $X p_{\text {Digiallibray }}$ Q}

[19] CORDIS. (2016) Lv dc microgrids for evolved energy communities. Accessed 07 Feb. 2018. [Online]. Available: https://cordis.europa.eu/project/rcn/201632_en.html.

[20] - (2017) Research, demonstration, and commercialisation of dc microgrid technologies. Accessed 07 Feb. 2018. [Online]. Available: https://cordis.europa.eu/ project/rcn/206774_en.html.

[21] IEC. (2017) Lvdc: electricity for the 21st century. Accessed 09 Feb. 2018. [Online]. Available: http://www.iec.ch/technologyreport/pdf/IEC_TR-LVDC.pdf.

[22] R. D. Middlebrook, "Input filter considerations in design and application of switching regulators," in Conf. Rec. IEEE IAS Annu. Meeting, 1976, pp. 366-382. 
[23] B. Nordman and K. Christensen, "Local power distribution with nanogrids," in 2013 International Green Computing Conference Proceedings, June 2013, pp. 1-8.

IEEEXPlore:

[24] T. Dragičević, X. Lu, J. C. Vasquez, and J. M. Guerrero, "Dc microgrids part ii: A review of power architectures, applications, and standardization issues," IEEE Trans. Power Electron., vol. 31, no. 5, pp. 3528-3549, May 2016.

IEEEXPlore:

[25] A. Kwasinski and C. N. Onwuchekwa, "Dynamic behavior and stabilization of dc microgrids with instantaneous constant-power loads," IEEE Transactions on Power Electronics, vol. 26, no. 3, pp. 822-834, March 2011.

IEEEXPlore $Q$

[26] H. Kakigano, Y. Miura, and T. Ise, "Low-voltage bipolar-type dc microgrid for super high quality distribution," IEEE Transactions on Power Electronics, vol. 25, no. 12, pp. 3066-3075, Dec 2010.

IEEEXPlore* $R$

[27] R. S. Balog and P. T. Krein, "Bus selection in multibus dc microgrids," IEEE Transactions on Power Electronics, vol. 26, no. 3, pp. 860-867, March 2011.

IEEEXPlore $Q$

[28] J. D. Park, J. Candelaria, L. Ma, and K. Dunn, "Dc ring-bus microgrid fault protection and identification of fault location," IEEE Transactions on Power Delivery, vol. 28, no. 4, pp. 2574-2584, Oct 2013.

IEEE Xp lore: $Q$

[29] R. M. Cuzner and G. Venkataramanan, "The status of dc micro-grid protection," in 2008 IEEE Industry Applications Society Annual Meeting, Oct 2008, pp. 1-8.

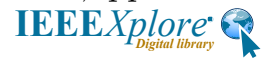

[30] D. Boroyevich, I. Cvetkovic, D. Dong, R. Burgos, F. Wang, and F. Lee, in Proc. Int. Conf. Opt. Electr. Electron. Equip., May 2010, pp. 1369-1380.

IEEEXP $X$ Pligitarlibrary

[31] E. Rodriguez-Diaz, F. Chen, J. C. Vasquez, J. M. Guerrero, R. Burgos, and D. Boroyevich, "Voltage-level selection of future two-level lvdc distribution grids: A compromise between grid compatibiliy, safety, and efficiency," IEEE Electrification Magazine, vol. 4, no. 2, pp. 20-28, June 2016.

IEEEXPlore: $Q$

[32] B. Subudhi and R. Pradhan, "A comparative study on maximum power point tracking techniques for photovoltaic power systems," IEEE Transactions on Sustainable Energy, vol. 4, no. 1, pp. 89-98, Jan 2013.

IEEEXP $p_{\text {Digial libray }}$ lore

[33] J. Schonberger, R. Duke, and S. D. Round, "DC-Bus Signaling: A Distributed Control Strategy for a Hybrid Renewable Nanogrid," IEEE Trans. Ind. Electron., vol. 53, no. 5, pp. 1453-1460, 2006.

IEEE $X P_{\text {Digialitibray }}$ lore 
[34] R. Olfati-Saber, J. A. Fax, and R. M. Murray, "Consensus and cooperation in networked multi-agent systems," Proceedings of the IEEE, vol. 95, no. 1, pp. 215-233, Jan 2007.

IEEE Xplore

[35] L. Meng, T. Dragicevic, J. Roldán-Pérez, J. C. Vasquez, and J. M. Guerrero, "Modeling and sensitivity study of consensus algorithmbased distributed hierarchical control for dc microgrids," IEEE Transactions on Smart Grid, vol. 7, no. 3, pp. 1504-1515, May 2016.

IEEE $X p_{\text {Diginal libray }}$ Q

[36] J. Guerrero, J. Vasquez, and R. Teodorescu, "Hierarchical control of droopcontrolled dc and ac microgrids 2014; a general approach towards standardization," in Proc. IEEE Annu. Conf. Ind. Electron. Soc., Nov 2009, pp. 4305-4310.

IEEE $X p_{\text {Digital libruy }}$ Q

[37] T. Dragičević, X. Lu, J. C. Vasquez, and J. M. Guerrero, "Dc microgrids - part i: A review of control strategies and stabilization techniques," IEEE Trans. Power Electron., vol. 31, no. 7, pp. 4876-4891, July 2016.

IEEE $X$ ppligial library $^{*} \mathrm{P}$

[38] A. Frances, R. Asensi, O. Garcia, R. Prieto, and J. Uceda, "Modeling electronic power converters in smart dc microgrids - an overview," IEEE Transactions on Smart Grid, 2017, early access.

IEEE Xplore* $Q$

[39] G. W. Wester and R. D. Middlebrook, "Low-frequency characterization of switched dc-dc converters," IEEE Trans. Aerosp. Electron. Syst, vol. AES-9, no. 3, pp. 376-385, May 1973.

IEEEXPlore: Q

[40] R. D. Middlebrook and S. Cuk, "A general unified approach to modelling switching-converter power stages," in Proc. IEEE Annu. Power Electron. Spec. Conf., June 1976, pp. 18-34.

IEEE $X p_{\text {Digitul library }}$ R

[41] S. Cuk and R. D. Middlebrook, "A general unified approach to modelling switching dc-to-dc converters in discontinuous conduction mode," in Proc. IEEE Annu. Power Electron. Spec. Conf., June 1977, pp. 36-57.

IEEE Xplore $Q$

[42] S. P. Hsu, A. Brown, L. Rensink, and R. D. Middlebrook, "Modelling and analysis of switching dc-to-dc converters in constant-frequency current-programmed mode," in Proc. IEEE Annu. Power Electron. Spec. Conf., June 1979, pp. 284-301.

IEEE Xplore*

[43] V. Vorperian, "Simplified analysis of pwm converters using model of pwm switch. continuous conduction mode," IEEE Trans. Aerosp. Electron. Syst, vol. 26, no. 3, pp. 490-496, May 1990. 
[44] - "Simplified analysis of pwm converters using model of pwm switch. ii. discontinuous conduction mode," IEEE Trans. Aerosp. Electron. Syst, vol. 26, no. 3, pp. 497-505, May 1990.

IEEEXPlore: $Q$

[45] Z. Zhang, Y. Y. Cai, Y. Zhang, D. J. Gu, and Y. F. Liu, "A distributed architecture based on microbank modules with self-reconfiguration control to improve the energy efficiency in the battery energy storage system," IEEE Trans. Power Electron., vol. 31, no. 1, pp. 304-317, Jan 2016.

IEEEXPlore $Q$

[46] L. Wang, D. Zhang, Y. Wang, B. Wu, and H. S. Athab, "Power and voltage balance control of a novel three-phase solid-state transformer using multilevel cascaded h-bridge inverters for microgrid applications," IEEE Trans. Power Electron., vol. 31, no. 4, pp. 3289-3301, April 2016.

IEEEXP $p_{\text {Digial libray }}$ ?

[47] S. R. Sanders, J. M. Noworolski, X. Z. Liu, and G. C. Verghese, "Generalized averaging method for power conversion circuits," IEEE Trans. Power Electron., vol. 6, no. 2, pp. 251-259, Apr 1991.

IEEEXPlore: $R$

[48] V. A. Caliskan, O. C. Verghese, and A. M. Stankovic, "Multifrequency averaging of dc/dc converters," IEEE Trans. Power Electron., vol. 14, no. 1, pp. 124-133, Jan 1999.

IEEEXP $X$ Digrialibray $Q$

[49] A. Emadi, "Modeling and analysis of multiconverter dc power electronic systems using the generalized state-space averaging method," IEEE Trans. Ind. Electron., vol. 51, no. 3, pp. 661-668, June 2004.

IEEEXP $X$ Digiarlibrary

[50] M. Daryabak, S. Filizadeh, J. Jatskevich, A. Davoudi, M. Saeedifard, V. K. Sood, J. A. Martinez, D. Aliprantis, J. Cano, and A. Mehrizi-Sani, "Modeling of lcc-hvdc systems using dynamic phasors," IEEE Trans. Power Del., vol. 29, no. 4, pp. 1989-1998, Aug 2014.

IEEEXPlore: P

[51] U. Javaid and D. Dujić, "Arbitrary order generalized state space average modeling of switching converters," in Proc. IEEE Energy Conversion Congr. Exposition, Sept 2015, pp. 6399-6406.

IEEEXplore: $Q$

[52] W. Dai, "Modeling and efficiency-based control of interleaved llc converters for pv dc microgrid," in Proc. IEEE Ind. Applicat. Soc. Annu. Meeting, Oct 2015, pp. 1-8.

IEEE Xplore $\mathrm{Q}$

[53] P. T. Krein, J. Bentsman, R. M. Bass, and B. L. Lesieutre, "On the use of averaging for the analysis of power electronic systems," IEEE Trans. Power Electron., vol. 5, no. 2, pp. 182-190, Apr 1990. 
[54] B. Lehman and R. M. Bass, "Extensions of averaging theory for power electronic systems," IEEE Trans. Power Electron., vol. 11, no. 4, pp. 542-553, Jul 1996.

IEEEXplore: $R$

[55] J. W. Kimball and P. T. Krein, "Singular perturbation theory for $\mathrm{dc} / \mathrm{dc}$ converters and application to pfc converters," IEEE Trans. Power Electron., vol. 23, no. 6, pp. 2970-2981, Nov 2008.

IEEEXPlore: $R$

[56] H. Behjati, L. Niu, A. Davoudi, and P. L. Chapman, "Alternative timeinvariant multi-frequency modeling of pwm dc-dc converters," IEEE Trans. Circuits Syst. I, Reg. Papers, vol. 60, no. 11, pp. 3069-3079, Nov 2013.

IEEE $X p_{\text {Digial library }}$ Q

[57] M. P. Foster, H. I. Sewell, C. M. Bingham, D. A. Stone, D. Hente, and D. Howe, "Cyclic-averaging for high-speed analysis of resonant converters," IEEE Trans. Power Electron., vol. 18, no. 4, pp. 985-993, July 2003.

IEEEXPlore $R$

[58] D. J. Shortt and F. c. Lee, "Improved switching converter model using discrete and averaging techniques," IEEE Trans. Aerosp. Electron. Syst, vol. AES-19, no. 2, pp. 190-202, March 1983.

IEEE $X p_{\text {Digitul library }}$ Q

[59] G. C. Verghese, M. E. Elbuluk, and J. G. Kassakian, "A general approach to sampled-data modeling for power electronic circuits," IEEE Trans. Power Electron., vol. PE-1, no. 2, pp. 76-89, April 1986.

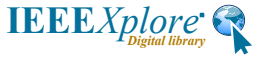

[60] F. Krismer and J. W. Kolar, "Accurate small-signal model for the digital control of an automotive bidirectional dual active bridge," IEEE Trans. Power Electron., vol. 24, no. 12, pp. 2756-2768, Dec 2009.

IEEEXPlore: $R$

[61] L. Scandola, L. Corradini, and G. Spiazzi, "Small-signal modeling of uniformly sampled phase-shift modulators," IEEE Trans. Power Electron., vol. 30, no. 10, pp. 5870-5880, Oct 2015.

IEEEXPlore $R$

[62] D. Maksimovic and R. Zane, "Small-signal discrete-time modeling of digitally controlled pwm converters," IEEE Trans. Power Electron., vol. 22, no. 6, pp. 2552-2556, Nov 2007. IEEEX $p_{\text {Digital libray }}$ Q

[63] L. Meng, T. Dragicevic, J. Roldán-Pérez, J. C. Vasquez, and J. M. Guerrero, "Modeling and sensitivity study of consensus algorithm-based distributed hierarchical control for dc microgrids," IEEE Trans. Smart Grid, vol. 7, no. 3, pp. 1504-1515, May 2016.

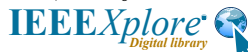

[64] M. K. Zadeh, R. Gavagsaz-ghoachani, J. p. Martin, S. Pierfederici, B. Nahid-Mobarakeh, and M. Molinas, "Discrete-time tool 
for stability analysis of dc power electronics based cascaded systems," IEEE Trans. Power Electron., vol. PP, no. 99, pp. 1-1, 2016.

IEEEXPlore: $R$

[65] R. M. Bass and J. Sun, "Large-signal averaging methods under large ripple conditions [for power convertors]," in Proc. IEEE Annu. Power Electron. Spec. Conf., vol. 1, May 1998, pp. 630-632 vol.1.

IEEEXPlore: $R$

[66] A. A. A. Radwan and Y. A. R. I. Mohamed, "Linear active stabilization of converter-dominated dc microgrids," IEEE Trans. Smart Grid, vol. 3, no. 1, pp. 203-216, March 2012.

IEEEXP $X$ Digritlibray $Q$

[67] S. Bae and A. Kwasinski, "Dynamic modeling and operation strategy for a microgrid with wind and photovoltaic resources," IEEE Trans. Smart Grid, vol. 3, no. 4, pp. 1867-1876, Dec 2012.

IEEEXPlore* $R$

[68] S. Anand and B. G. Fernandes, "Reduced-order model and stability analysis of low-voltage dc microgrid," IEEE Trans. Ind. Electron., vol. 60, no. 11, pp. 5040-5049, Nov 2013.

IEEEXPlore: $R$

[69] F. Zhao, N. Li, Z. Yin, and X. Tang, "Small-signal modeling and stability analysis of dc microgrid with multiple type of loads," in Proc. IEEE Power Syst. Technology Conf., Oct 2014, pp. 3309-3315.

IEEEXplore: R

[70] S. Liu, W. Zhu, Y. Cheng, and B. Xing, "Modeling and small-signal stability analysis of an islanded dc microgrid with dynamic loads," in Proc. IEEE Environment Elect. Eng. Int. Conf., June 2015, pp. 866-871.

IEEEXPlore: $Q$

[71] P. Xuewei and A. K. Rathore, "Small-signal analysis of naturally commutated current-fed dual active bridge converter and control implementation using cypress psoc," IEEE Trans. Veh. Technol., vol. 64, no. 11, pp. 4996-5005, Nov 2015.

IEEEXP Digialitibray $\mathrm{Q}$

[72] X. Lu, K. Sun, J. M. Guerrero, J. C. Vasquez, L. Huang, and J. Wang, "Stability enhancement based on virtual impedance for dc microgrids with constant power loads," IEEE Trans. Smart Grid, vol. 6, no. 6, pp. 2770-2783, Nov 2015.

IEEEXPlore:

[73] P. Shamsi and B. Fahimi, "Stability assessment of a dc distribution network in a hybrid micro-grid application," IEEE Trans. Smart Grid, vol. 5, no. 5, pp. 2527-2534, Sept 2014.

IEEEXPlore: $Q$

[74] S. Kazemlou and S. Mehraeen, "Decentralized discrete-time adaptive neural network control of interconnected dc distribution system," 
IEEE Trans. Smart Grid, vol. 5, no. 5, pp. 2496-2507, Sept 2014.

IEEE Xplore*

[75] S. Sanchez and M. Molinas, "Degree of influence of system states transition on the stability of a dc microgrid," IEEE Trans. Smart Grid, vol. 5, no. 5, pp. 2535-2542, Sept 2014 .

IEEE $X p_{\text {Digial libray }}$ Q

[76] P. G. Maranesi, V. Tavazzi, and V. Varoli, "Two-part characterization of pwm voltage regulators at low frequencies," IEEE Trans. Ind. Electron., vol. 35, no. 3, pp. 444-450, Aug 1988.

IEEEXPlore: $Q$

[77] B. H. Cho and F. C. Y. Lee, "Modeling and analysis of spacecraft power systems," IEEE Trans. Power Electron., vol. 3, no. 1, pp. 44-54, Jan 1988.

IEEEXPlore: $R$

[78] T. Suntio, M. Hankaniemi, and M. Karppanen, "Analysing the dynamics of regulated converters," IET IEE Proc. Electric Power Applicat., vol. 153, no. 6, pp. 905-910, November 2006.

IEEE $X p_{\text {Digiallibrary }}$ P

[79] M. Hankaniemi, M. Karppanen, T. Suntio, A. Altowati, and K. Zenger, "Source-reflected load interactions in a regulated converter," in Proc. IEEE Annu. Conf. Ind. Electron. Soc., Nov 2006, pp. 2893-2898.

IEEE Xplore $Q$

[80] M. Veerachary and A. R. Saxena, "G-parameter based stability analysis of dc-dc power electronic system," in Proc. IEEE Joint Int. Conf. Power Syst. Technology Power India Conf., Oct 2008, pp. 1-4.

IEEE Xplore $Q$

[81] J. Leppäaho, J. Huusari, L. Nousiainen, and T. Suntio, "Dynamics of current-fed converters and stability-assessment of solar-generator interfacing," in Proc. IEEE Int. Power Electron. Conf., June 2010, pp. 703-709.

IEEE Xplore $R$

[82] S. Vesti, T. Suntio, J. A. Oliver, R. Prieto, and J. A. Cobos, "Impedancebased stability and transient-performance assessment applying maximum peak criteria," IEEE Trans. Power Electron., vol. 28, no. 5, pp. 2099-2104, May 2013.

IEEEXPlore:

[83] W.-K. Chen, F. Bashir, S. Khanvilkar, A. Khokhar, and D. Schonfeld, The Electrical Engineering Handbook, 2005. [Online]. Available: $\quad$ http://www.sciencedirect.com/science/article/pii/B9780121709600500323.

IEEEXPlore: $R$

[84] S. F. Glover, "Modeling and stability analysis of a power electronics based systems," Ph.D. dissertation, Dept. Elect. Eng., Purdue Univ., Lafayette, IN, 2003. 
[85] K. Mino, J. Rico, and E. Barrera, "Modelling and simulation of power electronic converters using the component connection model," in Proc. IEEE Int. Midwest Symp. Circuits Syst., Aug 2009, pp. 921-928.

IEEEXPlore: $Q$

[86] A. Gelb and W. Vander Velde, Multiple-input describing functions and nonlinear system design, ser. McGraw-Hill electronic sciences series. McGraw-Hill, 1968.

[87] S. C. Chung, S. R. Huang, and C. I. Lin, "Applications of describing functions to estimate the continuous and discontinuous conduction mode for a dc-to-dc buck converter," IET IEE Proc. Electric Power Applicat., vol. 147, no. 6, pp. 513-519, Nov 2000.

IEEEXPlore:

[88] J. Shang, H. Li, X. You, T. Q. Zheng, and S. Wang, "A novel stability analysis approach based on describing function method using for dc-dc converters," in Proc. Appl. Power Electron. Conf. Expo., March 2015, pp. 2642-2647.

IEEEXPlore: $Q$

[89] M. S. Branicky, "Studies in Hybrid Systems: Modeling, Analysis, and Control," Ph.D. dissertation, MIT, Cambridge, 1995.

[90] H. Molla-Ahmadian, A. Karimpour, N. Pariz, and F. Tahami, "Hybrid modeling of a dc-dc series resonant converter: Direct piecewise affine approach," IEEE Trans. Circuits Syst. I, Reg. Papers, vol. 59, no. 12, pp. 3112-3120, Dec 2012.

IEEE Xplore $Q$

[91] T. Geyer, G. Papafotiou, and M. Morari, "Hybrid model predictive control of the step-down $\mathrm{dc} / \mathrm{dc}$ converter," IEEE Trans. Control Syst. Technol., vol. 16, no. 6, pp. 1112-1124, Nov 2008.

IEEEXPlore: $Q$

[92] F. M. Oettmeier, J. Neely, S. Pekarek, R. DeCarlo, and K. Uthaichana, "Mpc of switching in a boost converter using a hybrid state model with a sliding mode observer," IEEE Trans. Ind. Electron., vol. 56, no. 9, pp. 3453-3466, Sept 2009.

IEEEXPlore: $Q$

[93] C. Hoffmann and H. Werner, "A survey of linear parameter-varying control applications validated by experiments or high-fidelity simulations," IEEE Trans. Control Syst. Technol., vol. 23, no. 2, pp. 416-433, March 2015.

IEEEXPlore:

[94] S. Boyd, L. El Ghaoui, E. Feron, and V. Balakrishnan, Linear Matrix Inequalities in System and Control Theory, S. for Industrial and A. Mathematics, Eds., 1994.

[95] S. Sudhoff, S. Glover, S. Zak, S. Pekarek, E. Zivi, D. Delisle, and D. Clayton, "Stability Analysis Methodologies for DC Power Distribution Systems," in Ship Control Syst. Symp., 2003, pp. 1-10.

[96] T. Takagi and M. Sugeno, "Fuzzy identification of systems and its applications to modeling and control," IEEE Trans. Syst. Man Cybern., vol. SMC-15, no. 1, pp. 116-132, Jan 1985. 
[97] K. Mehran, D. Giaouris, and B. Zahawi, "Modeling and stability analysis of dc-dc buck converter via takagi-sugeno fuzzy approach," in Proc. IEEE Intelligent Syst. Knowledge Eng., vol. 1, Nov 2008, pp. 401-406.

IEEE Xplore: Q

[98] A. Kumar, A. S. Vempati, and L. Behera, "T-s fuzzy model based maximum power point tracking control of photovoltaic system," in Proc. IEEE Fuzzy Syst., July 2013, pp. 1-8.

IEEE $X p_{\text {Digial libray }}$ Q

[99] R. J. Wai and L. C. Shih, "Adaptive fuzzy-neural-network design for voltage tracking control of a dc/dc boost converter," IEEE Trans. Power Electron., vol. 27, no. 4, pp. 2104-2115, April 2012.

IEEEXPlore $Q$

[100] M. Luzar, M. Witczak, M. Mrugalski, and Z. Kanski, "Robust fault identification of a polytopic lpv system with neural network," in Proc. IEEE Int. Symp. Intelligent Control, Oct 2014, pp. 1614-1619.

IEEEXPlore*

[101] L. Arnedo, D. Boroyevich, R. Burgos, and F. Wang, "Un-terminated frequency response measurements and model order reduction for black-box terminal characterization models," in Proc. Appl. Power Electron. Conf. Expo., Feb 2008, pp. 1054-1060.

IEEE $X p_{\text {Digitall library }}$ R

[102] I. Cvetkovic, D. Boroyevich, P. Mattavelli, F. Lee, and D. Dong, "Unterminated, low-frequency terminal behavioral model of dc-dc converters," in Proc. Appl. Power Electron. Conf. Expo., March 2011, pp. 1873-1880.

IEEE $X p_{\text {Digiallibrary }} \mathrm{Q}$

[103] S. Vesti, J. A. Oliver, R. Prieto, J. A. Cobos, and T. Suntio, "Stability and transient performance assessment in a cots-module-based distributed $\mathrm{dc} / \mathrm{dc}$ system," in Proc. IEEE Int. Telecommun. Energy Conf., Oct 2011, pp. 1-7.

IEEEXPlore: $Q$

[104] MathWorks, "System Identification Toolbox User's Guide," p. 1164, 2016. [Online]. Available: https://es.mathworks.com/help/pdf\{_\}doc/ident/ident.pdf.

[105] J. Oliver, R. Prieto, J. Cobos, O. Garcia, and P. Alou, "Hybrid wiener-hammerstein structure for grey-box modeling of dc-dc converters," in Proc. Appl. Power Electron. Conf. Expo., Feb 2009, pp. 280-285.

IEEE Xplore* $Q$

[106] L. Arnedo, D. Boroyevich, R. Burgos, and F. Wang, "Polytopic black-box modeling of dc-dc converters," in Proc. IEEE Annu. Power Electron. Spec. Conf., June 2008, pp. 1015-1021.

IEEEXplore $R$

[107] I. Cvetkovic, D. Boroyevich, P. Mattavelli, F. Lee, and D. Dong, "Nonlinear, hybrid terminal behavioral modeling of a dc-based nanogrid system," in Proc. Appl. Power Electron. Conf. Expo., March 2011, pp. 1251-1258.

IEEEXplore: $Q$ 
[108] V. Valdivia, A. Barrado, A. Roldan, C. Fernandez, and P. Zumel, "Black-box modeling of dc-dc converters based on transient response analysis and parametric identification methods," in Proc. Appl. Power Electron. Conf. Expo., Feb 2010, pp. 1131-1138.

IEEEXPlore: $Q$

[109] S. D. Sudhoff and J. M. Crider, "Advancements in generalized immittance based stability analysis of dc power electronics based distribution systems," in 2011 IEEE Electric Ship Technologies Symposium, April 2011, pp. 207-212.

IEEEXPlore:

[110] X. Feng, J. Liu, and F. C. Lee, "Impedance specifications for stable dc distributed power systems," IEEE Transactions on Power Electronics, vol. 17, no. 2, pp. 157-162, Mar 2002. IEEEXPlore:

[111] X. Feng, Z. Ye, K. Xing, F. C. Lee, and D. Borojevic, "Individual load impedance specification for a stable dc distributed power system," in Applied Power Electronics Conference and Exposition, 1999. APEC '99. Fourteenth Annual, vol. 2, Mar 1999, pp. 923-929 vol.2.

IEEEXP $X$ Digialitibray $: Q$

[112] D. Marx, P. Magne, B. Nahid-Mobarakeh, S. Pierfederici, and B. Davat, "Large signal stability analysis tools in dc power systems with constant power loads and variable power loads - a review," IEEE Transactions on Power Electronics, vol. 27, no. 4, pp. 1773-1787, April 2012.

IEEEXPlore: $Q$

[113] R. K. BRAYTON and J. K. MOSER, "A theory of nonlinear networks-i," Quarterly of Applied Mathematics, vol. 22, no. 1, pp. 1-33, 1964. [Online]. Available: http://www.jstor.org/stable/43635388.

[114] C. J. Sullivan, S. D. Sudhoff, E. L. Zivi, and S. H. Zak, "Methods of optimal lyapunov function generation with application to power electronic converters and systems," in 2007 IEEE Electric Ship Technologies Symposium, May 2007, pp. 267-274.

IEEEXPlore: $Q$

[115] W. Du, J. Zhang, Y. Zhang, and Z. Qian, "Stability criterion for cascaded system with constant power load," IEEE Transactions on Power Electronics, vol. 28, no. 4, pp. 1843-1851, April 2013.

IEEE $X$ plore:

[116] P. Magne, B. Nahid-Mobarakeh, and S. Pierfederici, "Dynamic consideration of dc microgrids with constant power loads and active damping system. a design method for fault-tolerant stabilizing system," IEEE Journal of Emerging and Selected Topics in Power Electronics, vol. 2, no. 3, pp. 562-570, Sept 2014.

IEEEXPlore: $R$

[117] S. Singh, V. Kumar, and D. Fulwani, "Mitigation of destabilising effect of cpls in island dc micro-grid using non-linear control," IET Power Electronics, vol. 10, no. 3, pp. 387-397, 2017.

IEEEXPlore: $R$ 
[118] L. Herrera, W. Zhang, and J. Wang, "Stability analysis and controller design of dc microgrids with constant power loads," IEEE Transactions on Smart Grid, vol. 8, no. 2, pp. 881-888, March 2017. IEEEXplore: $Q$

[119] A. Francés, R. Asensi, O. García, and J. Uceda, "A blackbox large signal lyapunov-based stability analysis method for power converter-based systems," in Proc. IEEE Workshop Control Modeling Power Electron., June 2016, pp. 1-6.

IEEEXPlore: $Q$

[120] A. Francés, R. Asensi, O. García, R. Prieto, and J. Uceda, "A Black-box Modeling Approach for DC Nanogrids," in Proc. Appl. Power Electron. Conf. Expo., 2016, pp. 1624-1631.

[121] Danfoss. (2017) Design guide - vlt automationdrive fc 301/302. Accessed 29 Aug. 2017. [Online]. Available: http://files.danfoss.com/documents/PE/MG33BF22.pdf.

[122] F. D. Freijedo, E. Rodriguez-Diaz, M. S. Golsorkhi, J. C. Vasquez, and J. M. Guerrero, "A root-locus design methodology derived from the impedance/admittance stability formulation and its application for lcl grid-connected converters in wind turbines," IEEE Trans. on Power Electron., vol. 32, no. 10, pp. 8218-8228, Oct 2017.

IEEE Xplore: $\mathrm{Q}$

[123] TDK-Lambda. (2017) Eza datasheet. Accessed 29 Aug. 2017. [Online]. Available: https://uk.tdk-lambda.com/KB/EZA-Datasheet.pdf.

[124] A. Anvari-Moghaddam, T. Dragicevic, L. Meng, B. Sun, and J. M. Guerrero, "Optimal planning and operation management of a ship electrical power system with energy storage system," in Proc. IEEE Annu. Conf. Ind. Electron. Soc., Oct 2016, pp. 2095-2099.

IEEE $X p_{\text {Digitul libray }}$ 